Si-96-15

June 20, 1996

\title{
Inclusive Particle Production in Hadronic Decays of the Z Boson at LEP I
}

\author{
Armin Böhrer \\ Universität-GH Siegen, Germany \\ armin.boehrer@cern.ch
}

\begin{abstract}
Inclusive particle production in hadronic events taken at the $\mathrm{Z}$ resonance with LEP I is reviewed. With approximately 20 million $\mathrm{Z}$ decays, the four LEP experiments have studied the fragmentation of partons into identified particles. The high statistics allows for a detailed study of this non-perturbative QCD process. In this review the measurements of the four experiments are collected and the momentum spectra are compared to Monte Carlo models. More detailed aspects, such as relative production rates, particle content in gluon jets, particle correlation etc., are also treated.
\end{abstract}

This review represents the 'Habilitationsschrift', which has been submitted to the Physics Department at the Universität-GH Siegen, D-57068 Siegen. 


\section{Contents}

1 Introduction $\quad 1$

2 Theoretical Framework 3

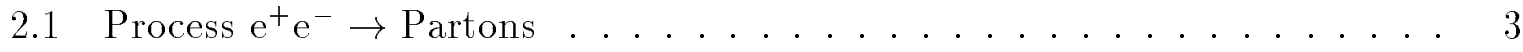

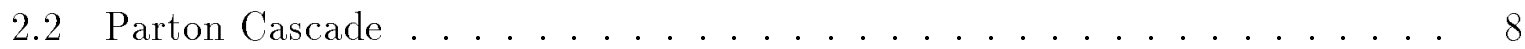

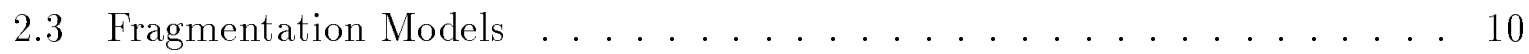

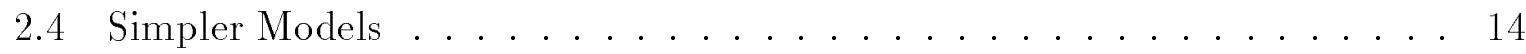

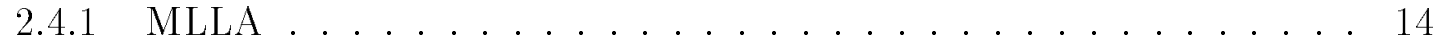

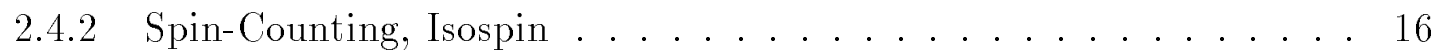

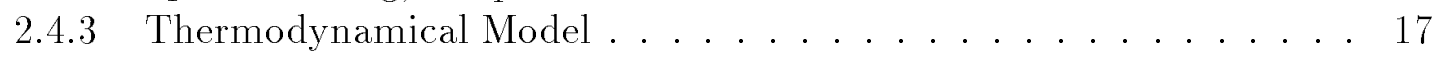

3 Data Taking $\quad \mathbf{1 8}$

3.1 LEP Collider . . . . . . . . . . . . . . . . . 18

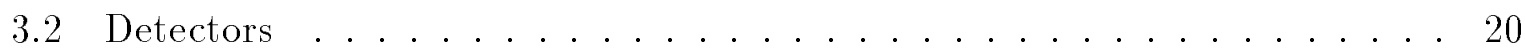

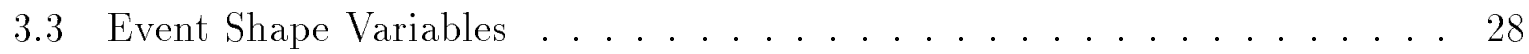

3.4 Model Tuning . . . . . . . . . . . . . . . . . . . . 30

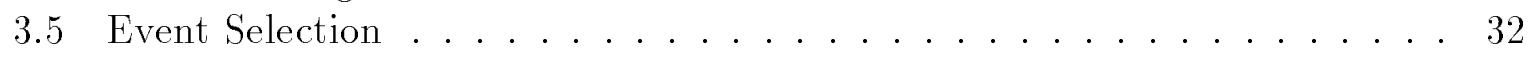

4 Particle Identification $\quad \mathbf{3 5}$

4.1 Detection Methods, Particle Identification ............. 35

4.2 Signal Extraction . . . . . . . . . . . . . . . . 37

5 Average Hadron Multiplicities $\quad 39$

5.1 Experimental Data . . . . . . . . . . . . . . . 39

5.2 Comparison with Monte Carlo Models . . . . . . . . . . . . 41

5.3 Measurements at Lower Centre-of-Mass Energy . . . . . . . . . . . . 43

6 Momentum Spectra $\quad 46$

6.1 Stable Particles . . . . . . . . . . . . . . . . 47

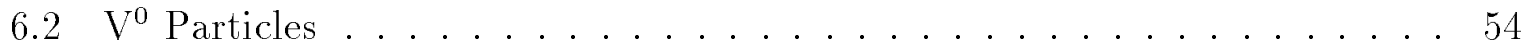

6.3 Particles Decaying to Photons . . . . . . . . . . . . . . . . 58

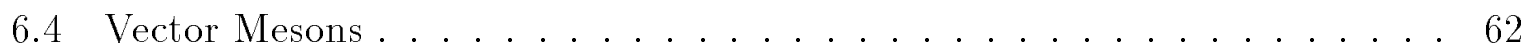

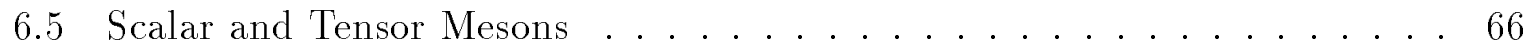

6.6 Non-Strange Baryons . . . . . . . . . . . . . . . . . 69

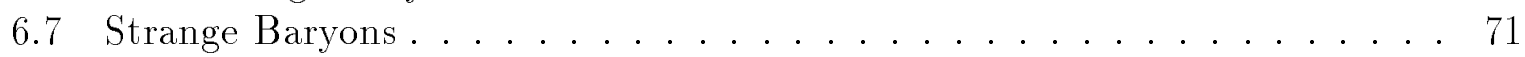


6.8 Charmed Hadrons . . . . . . . . . . . . . . . . . . . 75

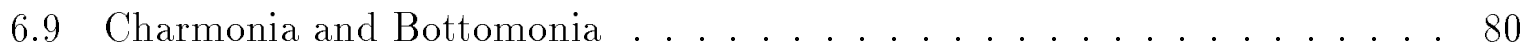

6.10 Bottom Hadrons . . . . . . . . . . . . . . . . 83

7 Related Results $\quad \mathbf{8 7}$

7.1 Comparisons with Models . . . . . . . . . . . . 87

7.2 Relative Rates . . . . . . . . . . . . . . . 90 90

7.3 Dependence on Centre-of-Mass Energy . . . . . . . . . . . . . 98

7.4 Particle Composition in Flavour Tagged Events . . . . . . . . . . . 101

7.5 Particle Composition in Quark and Gluon Jets . . . . . . . . . . . . . 106

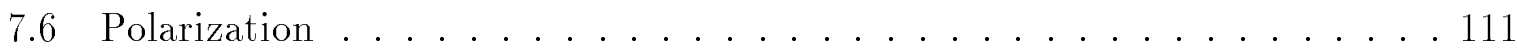

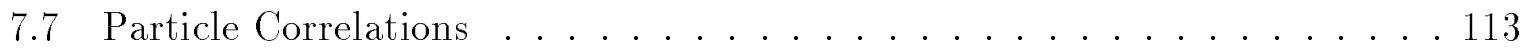

8 Conclusion and Outlook $\quad 121$

$\begin{array}{lr}\text { List of Figures } & 125\end{array}$

$\begin{array}{lr}\text { List of Tables } & 126\end{array}$ 


\section{Chapter 1}

\section{Introduction}

The formation of hadrons in the annihilation process $\mathrm{e}^{+} \mathrm{e}^{-} \rightarrow \mathrm{q} \overline{\mathrm{q}} \rightarrow$ hadrons is described by the Standard Model of electroweak interactions (quantum-flavour-dynamics, QFD) and the theory of strong interactions (quantum-chromo-dynamics, QCD). While the hard process, i.e., the formation of quarks and gluons, is calculable using perturbation theory, the transition from quarks and gluons to hadrons occurs with small momentum transfer where the strong coupling constant $\left(\alpha_{\mathrm{s}}\right)$ is large. This latter step can only be estimated non-perturbatively and is modelled using Monte Carlo generators (e.g., JETSET [1], HERWIG [2]).

The study of inclusive particle production is primarily for the investigation of hadronization. Data are needed for the understanding of this non-perturbative regime. They are used for improving, testing, and differentiating models, tuning their parameters (Refs. [3] to [7]). The $\mathrm{e}^{+} \mathrm{e}^{-}$annihilation is an ideal reaction, because of its well defined initial state without colour.

The four experiments at LEP have collected about 20 million hadronic $Z$ decays from their startup in 1989 to mid 1995, when the data taking at the $Z$ resonance was completed and the beam energy increased. The high cross section at the $Z$ resonance (Figure 1.1), and therefore high statistics, allows a detailed study of identified particles with higher precision than at lower energies. The high statistics also allows measurement of mesons and baryons with low production rates. (Throughout this review a specific state implies the inclusion of the antiparticle as well; particle multiplicities include the decay products of hadrons with lifetime $\tau<1$ ns.)

In this paper the data on identified particles are collected as measured by the four LEP experiments. In Chapter 2 a theoretical introduction to aspects of QCD, relevant for this report, is given. This includes a description of Monte Carlo generators as well as phenomenological models. Chapter 3 on data taking describes the experimental setup of the LEP experiments followed by the tuning of the models and the hadronic event selection. In Chapters 4 and 5 the principles of signal extraction of identified particles and an overview of the average particle multiplicities per hadronic $\mathrm{Z}$ decay in data and Monte Carlo are presented. These two Chapters can be considered as a short introduction to the more detailed presentation of Chapter 6 . Here methods of signal extraction are given; the spectra measured with the four LEP experiments are compared to model predictions. In 


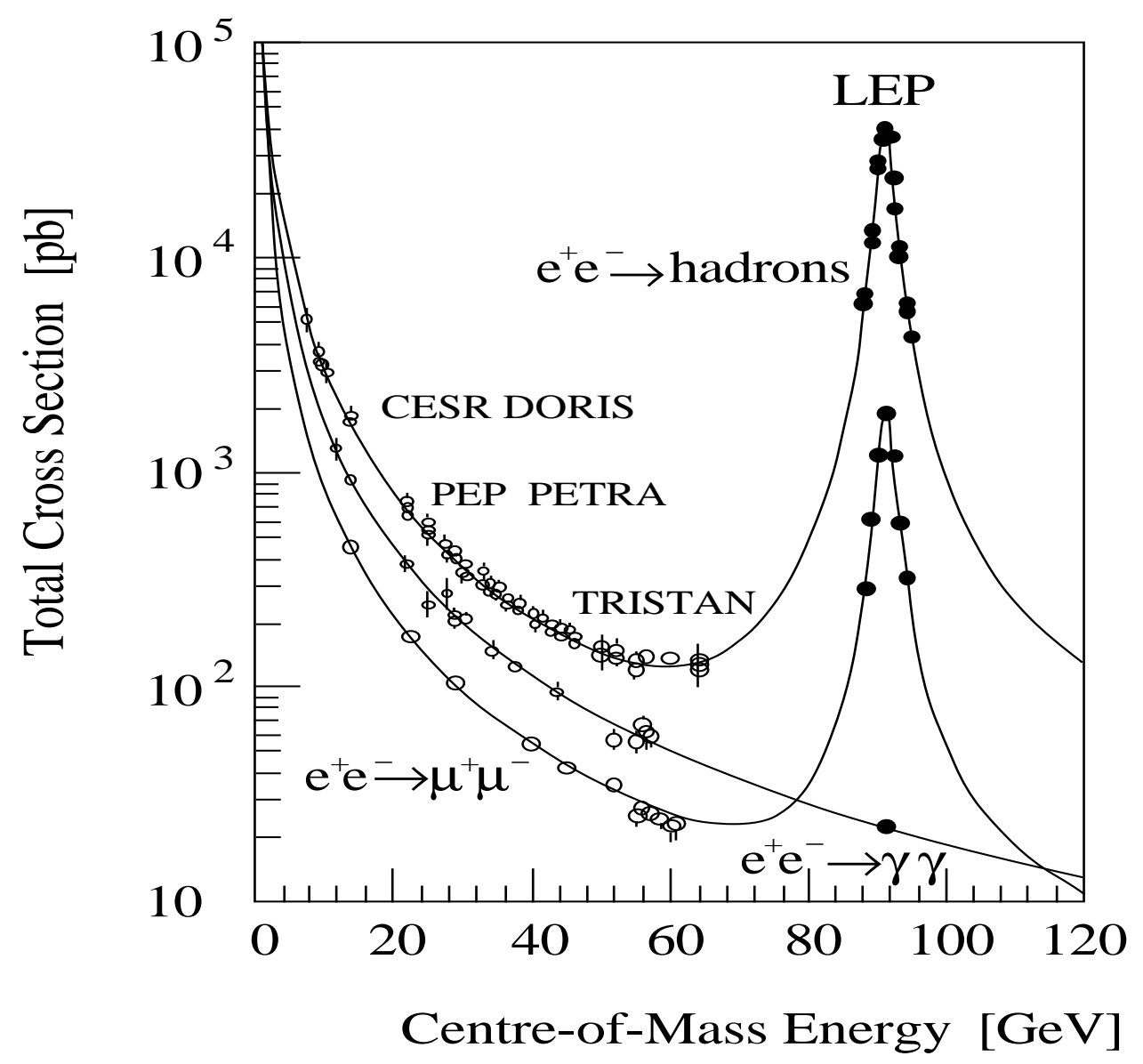

Figure 1.1: Cross section in $\mathrm{e}^{+} \mathrm{e}^{-}$annihilation as a function of centre-of-mass energy. Measurements are shown in comparison with the prediction of the Standard Model [64].

Chapter 7 more specific results, such as strangeness suppression and particle composition in tagged events, are presented. Finally in Chapter 8 the results are summarized and an outlook is given.

All four LEP experiments are treated, however, the author of the paper being a member of the ALEPH collaboration; in some aspects (detector description, model tuning) the ALEPH experiment may be more detailed or taken as an example.

The particle rates collected from References [8] to [61] are listed in Table 5.1 and 5.2 for the production rates (for the experiments and their averages), which will give a comprehensive overview of the particle production rates, together with the Figures in Chapter 6 for the momentum spectra.

For further reading, we recommend: a similar review for the discussion of data at lower energies, $10-40 \mathrm{GeV}[62]$; a compilation of $\mathrm{e}^{+} \mathrm{e}^{-}$data, giving production rates and tables of particle spectra [63]; a general overview of QCD results at LEP [64]. 


\section{Chapter 2}

\section{Theoretical Framework}

\subsection{Process $\mathrm{e}^{+} \mathrm{e}^{-} \rightarrow$ Partons}

The Z-production and decay of the Z-boson has been intensively studied at LEP ([65, 66, $67,68]$. From the measurements of the total cross sections of the reaction $\mathrm{e}^{+} \mathrm{e}^{-} \rightarrow \mathrm{f} \overline{\mathrm{f}}$, i.e., for the creation of a fermion pair, information on the Z-mass, partial and total widths are extracted. Quark pairs are found to be the dominant decay channel: $70 \%=\Gamma_{\text {had }} / \Gamma_{\text {tot }}$ are hadronic decays. Flavour tagging of bottom and charm quarks has been performed and their partial widths found to be $R_{\mathrm{b}}=\Gamma_{\mathrm{b} \overline{\mathrm{b}}} / \Gamma_{\text {had }}=0.2219 \pm 0.0017$ and $R_{\mathrm{c}}=\Gamma_{\mathrm{c} \overline{\mathrm{c}}} / \Gamma_{\text {had }}=$ $0.1540 \pm 0.0074$, respectively. These results can be compared to the expected values of the Standard Model of electroweak interactions. The measurement of the total hadronic width serves serves as (in principle) best determination of the strong coupling constant, because the prediction is calculated up to third order $\alpha_{\mathrm{s}}$.

The cross section for the production of a fermion pair at the $\mathrm{Z}$ pole is obtained, to lowest order, according to the quark-parton model ${ }^{1}$ as illustrated by the Born-graph in Figure 2.1.

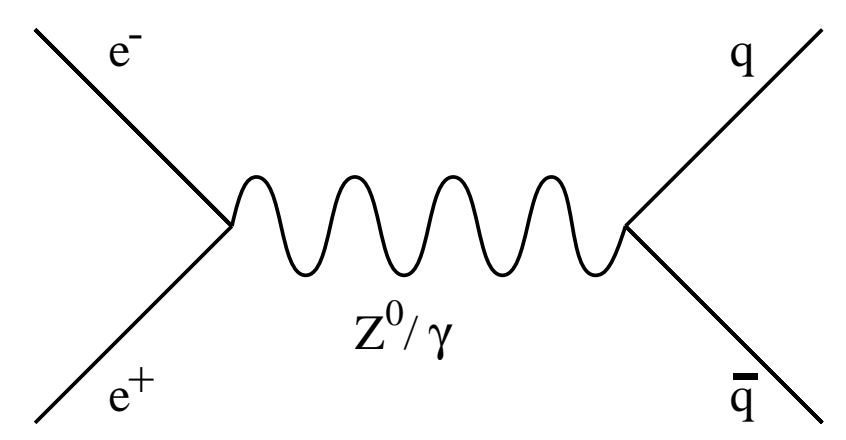

Figure 2.1: Lowest order contribution of $\mathrm{e}^{+} \mathrm{e}^{-}$annihilation into a quark-pair (Born-graph).

This purely electroweak process, the annihilation of an electron-positron pair via a

\footnotetext{
${ }^{1}$ Here, we closely follow the very nice review 'Handbook of perturbative QCD' [69].
} 
photon and $\mathrm{Z}$ vector meson into a different fermion pair, can be calculated using Feynman rules. The coupling at the fermion-vector vertex is $e Q_{i} \gamma^{\mu}$ for exchange of a photon, with $Q_{i}$ being the electric charge of the fermion. For the exchange of the $\mathrm{Z}$, the vertex factor is given by

$$
e \Gamma^{\mu}=\frac{e}{\sin \theta_{\mathrm{W}} \cos \theta_{\mathrm{W}}} \gamma^{\mu}\left(V_{f}-A_{f} \gamma_{5}\right),
$$

with the weak mixing angle $\theta_{\mathrm{W}}$. The vector and axial couplings are defined with the weak isospin $I_{3}$ as

$$
A_{f}=a_{f} \cdot 2 \sin \theta_{\mathrm{W}} \cos \theta_{\mathrm{W}}=I_{3}-2 Q_{f} \sin ^{2} \theta_{\mathrm{W}}
$$

and

$$
V_{f}=v_{f} \cdot 2 \sin \theta_{\mathrm{W}} \cos \theta_{\mathrm{W}}=I_{3} .
$$

At centre-of-mass energies above the production threshold of the Upsilon, the five flavours $\mathrm{u}, \mathrm{d}, \mathrm{s}, \mathrm{c}$, and $\mathrm{b}$, with their masses small enough for pair production, contribute to the hadronic cross section in $\mathrm{e}^{+} \mathrm{e}^{-}$annihilation. The top-quark is known to be too heavy for production at the present $\mathrm{e}^{+} \mathrm{e}^{-}$colliders, $m_{\text {top }} \approx 180 \pm 12 \mathrm{GeV} / c^{2}$ [70]. The total hadronic cross section at energies $\sqrt{s}$ well below the $\mathrm{Z}$ resonance is given by the photon exchange alone:

$$
\sigma(s)_{t o t}=\frac{4 N_{c} \pi \alpha^{2}}{3 s} \sum_{f} Q_{f}^{2},
$$

where the sum runs over the five flavours; $\alpha=e^{2} / 4 \pi$ is the electromagnetic fine-structure constant. The number of colours $N_{c}$ is known and has been measured from the ratio of the hadronic to the muon-pair cross section to be three, in agreement with the expectation of the quark-parton model of three colours and fractional charges $Q_{f}$ for the quarks.

At energies around $m_{\mathrm{Z}}=91.1884 \pm 0.0022 \mathrm{GeV} / c^{2}[68]$, the electro-weak cross section, including $\mathrm{Z}$ exchange and $\mathrm{Z} \gamma$-interference becomes

$$
\sigma(s)_{t o t}=\frac{4 N_{c} \pi \alpha^{2}}{3 s} \sum_{f} Q_{f}^{2}\left(1-2 \chi V_{f}^{2}+\left[V_{f}^{2}+A_{f}^{2}\right]^{2} \chi^{2}\right) .
$$

Again, the sum runs over the five flavours and $\chi$ is defined as

$$
\chi=\frac{s}{s-m_{\mathrm{Z}}^{2}} \frac{1}{4 \sin \theta_{\mathrm{W}} \cos \theta_{\mathrm{W}}} .
$$

The parton model provides even more information on the subject of this paper, the single-hadron inclusive cross section, when a hadron $\mathrm{h}$ with momentum $p$ is produced:

$$
\mathrm{e}^{+} \mathrm{e}^{-} \rightarrow \mathrm{h}(p)+\mathrm{X}
$$

where X denotes the system of all other particles in the final state (Figure 2.2).

Readers familiar with deep inelastic scattering will realize, that the diagram in Figure 2.2 is the 'crossed' amplitude of the inelastic scattering of an electron with the antiparticle $\bar{h}$. The hadron $h$ is transferred from the final state into the initial state, while the incoming positron becomes an outgoing electron. 


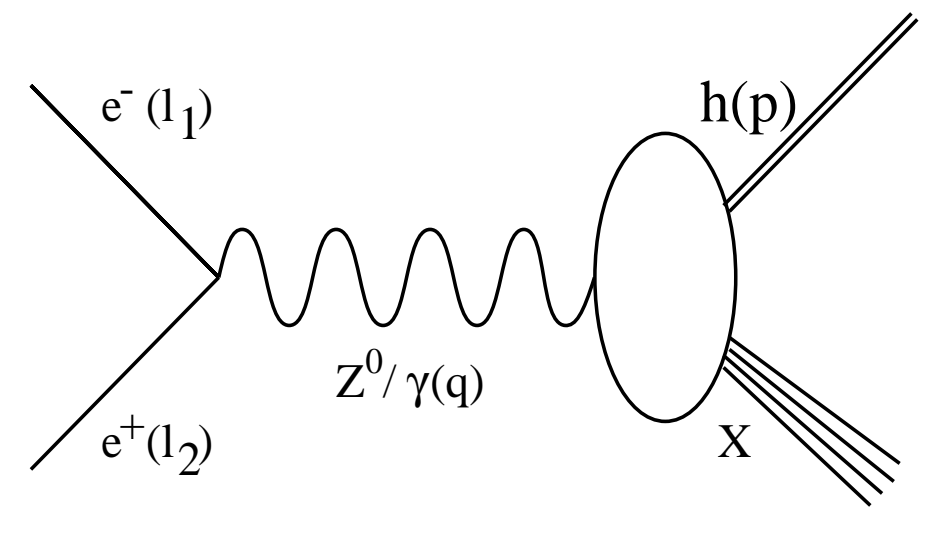

Figure 2.2: Inclusive single-hadron production.

The kinematics of the inclusive single-hadron production are given by similar variables as in deep inelastic scattering. The total four-momentum transferred is just the sum of the four-momenta $l_{i}$ of the incoming leptons:

$$
\begin{aligned}
q & =l_{1}+l_{2}, \\
q^{2} & =Q^{2}>0 .
\end{aligned}
$$

Measures for the energy and direction (angle $\theta_{p l_{1}}$ of the hadron with momentum $p$ and the electron with momentum $l_{1}$ ) are two dimensionless variables

$$
\begin{gathered}
x=\frac{2 p q}{q^{2}} \\
y=\frac{p l_{1}}{p q}=\frac{1}{2}\left(1-\cos \theta_{p l_{1}}\right) .
\end{gathered}
$$

in the centre-of-mass system $(\mathbf{q}=0)$.

In analogy to deep inelastic scattering (for simplicity only photon-exchange is taken), the cross section for inclusive single-hadron production can be written as a product of a leptonic tensor $L^{\mu \nu}$ and a hadronic tensor $\bar{W}_{\mu \nu}$ :

$$
\mathrm{d}^{2} \sigma_{\mathrm{e}^{+} \mathrm{e}^{-}}^{h}=\frac{1}{q^{2}} L_{e \gamma}^{\mu \nu}\left(l_{i}\right) \bar{W}_{\mu \nu}^{(\gamma h)}(p, q) \mathrm{d} x \mathrm{~d} y
$$

where $\bar{W}_{\mu \nu}$ is given by

$$
\bar{W}_{\mu \nu}^{(\gamma h)}(p, q)=-x\left(g_{\mu \nu}-\frac{q_{\mu} q_{\nu}}{q^{2}}\right) \bar{F}_{1}^{(\gamma h)}\left(x, q^{2}\right)+\left(p_{\mu}-q_{\mu} \frac{p q}{q^{2}}\right)\left(p_{\nu}-q_{\nu} \frac{p q}{q^{2}}\right) \frac{1}{m_{h}^{2}} \bar{F}_{2}^{(\gamma h)}\left(x, q^{2}\right)
$$

and $\bar{F}_{1}, \bar{F}_{2}$ are the single-hadron inclusive structure functions. We can write the cross section as

$$
\frac{\mathrm{d}^{2} \sigma_{\mathrm{e}^{+} \mathrm{e}^{-}}\left(x, y, q^{2}\right)}{\mathrm{d} x \mathrm{~d} y}=N_{c} \frac{4 \pi \alpha^{2}}{3 q^{2}}\left(\frac{3}{2} \bar{F}_{1}^{(\gamma h)}-3 y(1-y) \bar{F}_{2}^{(\gamma h)}\right) .
$$


In the parton model, this cross section can be written with the help of the Born cross section $\mathrm{d} \sigma_{\mathrm{e}^{+} \mathrm{e}^{-}}^{f}$ for production of a quark with flavour $f$ in $\mathrm{e}^{+} \mathrm{e}^{-}$annihilation and the quark fragmentation function $D_{f}^{(\gamma h)}$ into a hadron $h$ for this flavour:

$$
\frac{\mathrm{d}^{2} \sigma_{\mathrm{e}^{+} \mathrm{e}^{-}}\left(x, y, q^{2}\right)}{\mathrm{d} x \mathrm{~d} y}=\sum_{f} \int \mathrm{d} x^{\prime} \mathrm{d} z \frac{\mathrm{d} \sigma_{\mathrm{e}^{+} \mathrm{e}^{-}}^{f}\left(x^{\prime}, y, q^{2}\right)}{\mathrm{d} x^{\prime} \mathrm{d} y} D_{f}^{(\gamma h)}(z) \delta\left(x^{\prime}-x / z\right) .
$$

The fragmentation function $D_{f}^{h}$ describes the probability that the quark with flavour $f$ produces a hadron with the fraction $z=\left(E+p_{l}\right)_{\text {hadron }} /\left(E+p_{l}\right)_{\text {quark }}$ of the momentum of the quark.

From this the relation

$$
\bar{F}_{f}^{(\gamma h)}(x)=Q_{f}^{2} D_{h / f}(x)
$$

can be derived in the framework of the parton model, connecting the single-hadron inclusive structure functions with the fragmentation functions. The cross section is then given as

$$
\frac{\mathrm{d}^{2} \sigma_{\mathrm{e}^{+} \mathrm{e}^{-}}^{h}\left(x, y, q^{2}\right)}{\mathrm{d} x \mathrm{~d} y}=N_{c} \frac{\pi \alpha^{2}}{q^{2}} \sum_{f} Q_{f}^{2}\left(1+\cos ^{2} \theta_{p l_{1}}\right) D_{h / f}(x) .
$$

The angular dependence $\left(1+\cos ^{2} \theta_{p l_{1}}\right)$ in the centre-of-mass system is due to the spin of $1 / 2$ of the quarks.

These considerations must be corrected for gluon emission. The total hadronic cross section is calculated up to third order [71] and we have $\sigma_{t o t}=\sigma_{0}\left(1+\delta_{Q C D}\right)$ (for $\gamma$ exchange):

$$
\begin{gathered}
\sigma_{\text {tot }}\left(Q^{2}\right)=\sigma_{0}\left(Q^{2}\right)\left[1+\frac{\alpha_{\mathrm{s}}}{\pi}+1.409\left(\frac{\alpha_{\mathrm{s}}}{\pi}\right)^{2}-12.805\left(\frac{\alpha_{\mathrm{s}}}{\pi}\right)^{3}\right] \\
\sigma_{0}\left(Q^{2}\right)=\frac{4 N_{c} \pi \alpha^{2}}{3 Q^{2}} \sum_{f} Q_{f}^{2} .
\end{gathered}
$$

Further corrections were calculated recently [72]. It is more convenient to translate $\sigma_{\text {tot }}$ into the ratio of hadronic to electronic width (for $\gamma$ and $Z$ exchange):

$$
R(Q C D)=\Gamma(\mathrm{Z} \rightarrow \mathrm{q} \overline{\mathrm{q}}) / \Gamma\left(\mathrm{Z} \rightarrow \mathrm{e}^{+} \mathrm{e}^{-}\right)=19.943\left[1+1.060 \frac{\alpha_{\mathrm{s}}}{\pi}+0.90\left(\frac{\alpha_{\mathrm{s}}}{\pi}\right)^{2}-15\left(\frac{\alpha_{\mathrm{s}}}{\pi}\right)^{3}\right],
$$

a parameterization, which also takes into account that $R$ does not factorize [73].

With the third order parameterization, $\alpha_{\mathrm{s}}\left(m_{\mathrm{Z}}\right)=0.126 \pm 0.007$ is obtained, a value slightly higher than the world average from other $\alpha_{\mathrm{s}}$ measurements; $\alpha_{\mathrm{s}}\left(m_{\mathrm{Z}}\right)=0.117 \pm 0.006$ in Ref. [74] or $\alpha_{\mathrm{s}}\left(m_{\mathrm{Z}}\right)=0.118 \pm 0.005$ in Ref. [64]. The difference may be due to the partial width of bottom quark production, where the measured value is 3 standard deviations higher than expected in the Standard Model. It has been pointed out recently [75] that a possible, but not really satisfactory, solution would be a lower $\alpha_{\mathrm{s}}$ value. Unknown new physics may be the reason or, much simpler, experimental uncertainties from the correlation to the partial charm width, which is measured smaller than expected.

For the inclusive single-hadron production, higher order QCD corrections (gluon emission) lead to the effect that the fragmentation function, which in the parton model is 


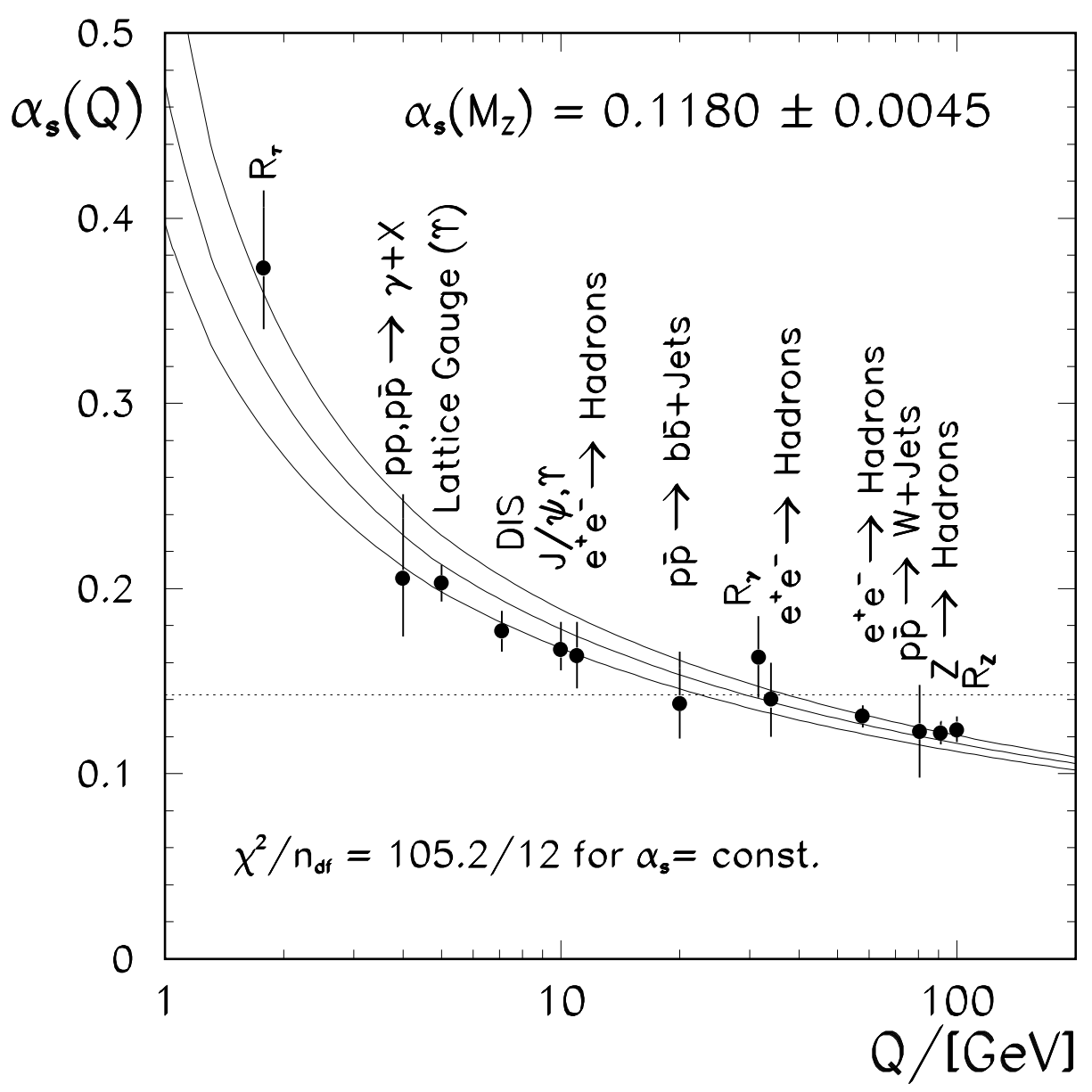

Figure 2.3: Compilation of measurements demonstrating the running of the strong coupling constant: the solid lines indicate the fit and one standard deviation errors for $\alpha_{\mathrm{s}}\left(m_{\mathrm{Z}}\right)=0.1180 \pm 0.0045$. The dotted line is the average of all measurement, i.e., assuming a constant $\alpha_{\mathrm{s}}[64]$.

independent of $\sqrt{s}$ (Feynman scaling), becomes dependent of the centre-of-mass energy. In addition to this gluon emission; the change in phase space, the difference in flavour composition and particle decays lead to a much more complicated situation, when looking at spectra of identified final state particles. Therefore, the explicit calculation of multihadron production is not completely solved. With $\alpha_{\mathrm{s}}$ being a function of the momentum transfer involved in the process only hard processes are precisely calculable. For that reason the process $\mathrm{e}^{+} \mathrm{e}^{-} \rightarrow$ hadrons is simulated using Monte Carlo methods, in order to compare theoretical predictions with experiment. (A discussion of various Monte Carlo models may be found in References [3, 65].)

Monte Carlo models [76] (such as JETSET or HERWIG) assume that the hadron production ( $\mathrm{e}^{+} \mathrm{e}^{-} \rightarrow$ hadrons) factorizes: in a first step the quark pair production is calculated including bremsstrahlung, then gluon radiation and gluon splitting is modelled, fragmentation or hadronization of partons to hadrons is simulated, which is then followed by 
particle decay. The measurements of identified particle spectra, the subject of this article, are used to verify the assumptions made in the transition from quarks and gluons to the final state hadrons and to improve the understanding of the non-perturbative part by adjusting model parameters.

While the quark pair production is calculable in a straightforward way, the other steps need some explanation; they shall be discussed in the following sections.

\subsection{Parton Cascade}

The matrix elements for the differential cross sections of parton production are calculated up to second order in $\alpha_{\mathrm{s}}$. It turns out, as had already been seen at PEP/PETRA energies, that the 4-jet rate is underestimated. Higher order contributions are important for the correct description of the shower and hadronization evolution. An improvement on the theoretical side is to use parton shower models (Figure 2.4), which are derived using the leading logarithmic approximation (LLA).

The LLA uses the fact that some quantities $R\left(\rho, \alpha_{\mathrm{s}}(s)\right)$ exponentiates, i.e., that their main contribution can be written as [77]

$$
R\left(\rho, \alpha_{\mathrm{s}}(s)\right) \propto \mathrm{e}^{\sum_{n=1}^{\infty} \alpha_{g}^{n} \sum_{m=1}^{n+1} G_{n m} \ln ^{m} \rho} .
$$

The terms with $m=n+1$ are called leading, with $m=n$ next-to-leading, and with $m<n$ sub-leading. Other approximations are known as MLLA (modified) and DLLA (double), depending on the way sub-leading terms are included.

This summation of leading logarithms is suitably formulated in a probabilistic framework, as required for Monte Carlo event simulation: in parton shower models the evolution of the parton shower cascade is looked upon as an arbitrary number of branchings of one parton into two. There is no upper limit on the number of partons as for the (fixed order) matrix element calculation. This provides a good description of the multi-jet structure of hadronic events. The models are usually matched to the first order matrix element to describe hard gluon radiation.

The probability $\mathcal{P}_{\mathrm{a} \rightarrow \mathrm{bc}}$ for the decay of a parton is a function of the evolution variable $Q^{2}$ (which may be chosen as the virtuality of the parton) and of $z$, which gives the sharing of the energy and momentum of the parent a between the daughters $b$ and $c$ with fraction $z$ and $1-z$, respectively. The Altarelli-Parisi equation

$$
\frac{\mathrm{d} \mathcal{P}_{\mathrm{a} \rightarrow \mathrm{bc}}}{\mathrm{d} t}=\int \mathrm{d} z \frac{\alpha_{\mathrm{s}}}{2 \pi} P_{\mathrm{a} \rightarrow \mathrm{bc}}(z), t=\ln \left(Q^{2} / \Lambda^{2}\right)
$$

can have three different kernels, describing gluon bremsstrahlung off a quark, gluon bremsstrahlung off a gluon and gluon splitting. The kernels are:

$$
\begin{gathered}
P_{\mathrm{q} \rightarrow \mathrm{qg}}(z)=C_{F} \frac{1+z^{2}}{1-z}, \quad C_{F}=\frac{4}{3} ; \\
P_{\mathrm{g} \rightarrow \mathrm{gg}}(z)=N_{c} \frac{(1-z(1-z))^{2}}{z(1-z)}, \quad N_{c}=3 ;
\end{gathered}
$$




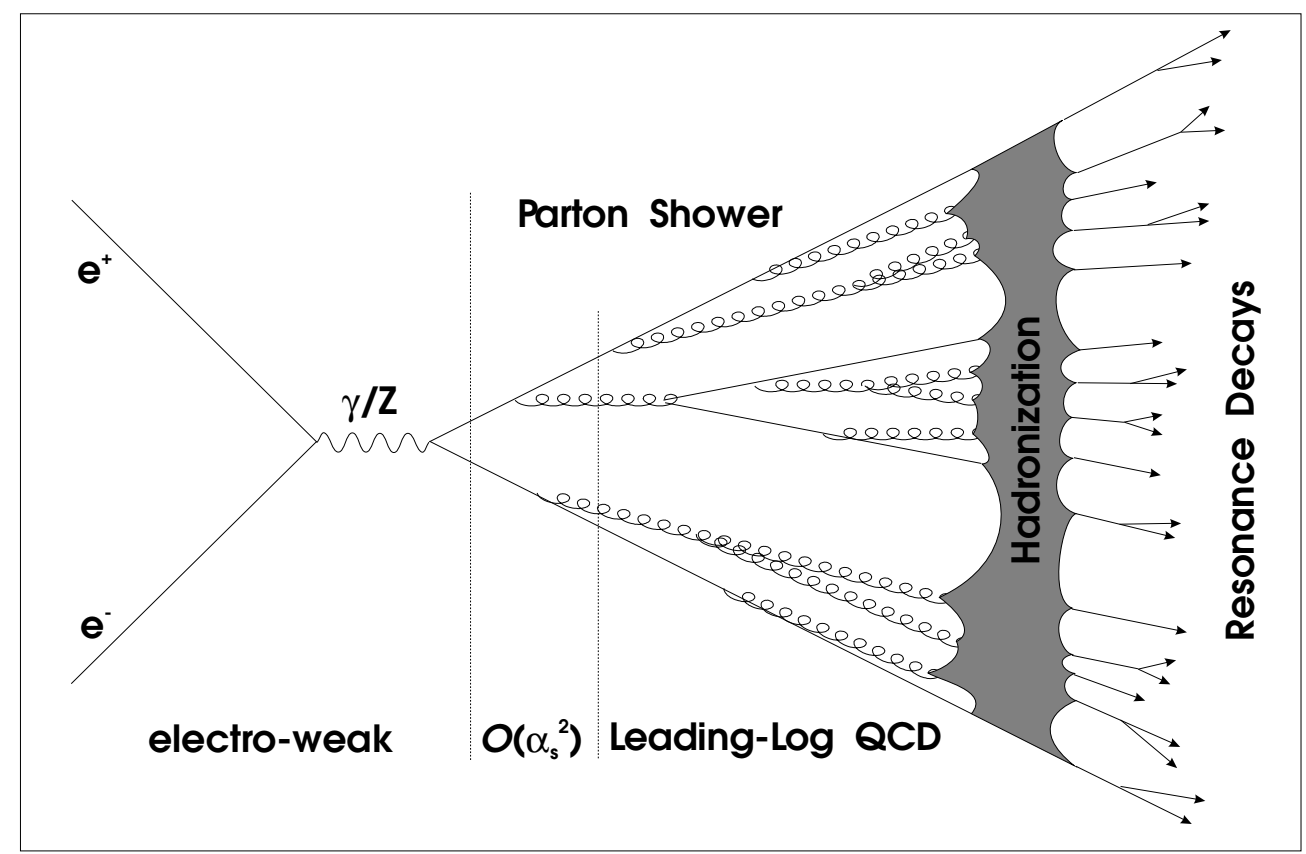

Figure 2.4: Schematic view of a parton shower in $\mathrm{e}^{+} \mathrm{e}^{-}$annihilation with subsequent hadronization and particle decays [64].

$$
P_{\mathrm{g} \rightarrow \mathrm{q} \overline{\mathrm{q}}}(z)=T_{R}\left(z^{2}+\left((1-z)^{2}\right), \quad T_{R}=\frac{n_{f}}{2} ;\right.
$$

where $C_{F}$ and $T_{R}$ are colour factors of QCD and $N_{c}$ is the number of colours. The strong coupling constant used is the first order one:

$$
\alpha_{\mathrm{s}}\left(Q^{2}\right)=\frac{12 \pi}{\left(33-2 n_{f}\right) \ln \left(Q^{2} / \Lambda^{2}\right)},
$$

where $\Lambda$ is the QCD scale parameter. In the leading logarithmic approximation $Q^{2}=$ $z(1-z) m_{\mathrm{a}}^{2}$ is chosen, which need not be the same as the evolution variable $\left(m_{\mathrm{a}}\right.$ is the mass of the parent parton). This is different from the choice made for matrix element calculations. The shower evolution ends, if the evolution variable $Q$ has dropped below a threshold $Q_{0}$, which is of $\mathcal{O}(1 \mathrm{GeV})$. The parton shower models differ in their definition of $Q$. In JETSET $Q^{2}=m_{\mathrm{a}}^{2}$ is used, while in HERWIG the evolution variable is defined as $Q^{2}=E_{\mathrm{a}}^{2}(1-\cos \theta)$. Here, $m_{\mathrm{a}}$ and $E_{\mathrm{a}}$ are the mass and the energy of the parent parton, and $\theta$ is the opening angle of the daughter partons. The choice of $Q$ made in HERWIG accounts for the coherence of soft gluons, simulated in the model by angular ordering, i.e. partons radiated at a later stage in the evolution branch with smaller angles. Angular ordering is introduced in JETSET by hand.

The translation of the shower evolution of massless partons into the probability picture 
in a Monte Carlo simulation causes further problems, such as avoiding divergences for the final state with a fixed number of partons.

\subsection{Fragmentation Models}

The transition from partons to hadrons is not yet understood from first principles in QCD. At small momentum transfer, the high coupling constant prevents an exact calculation. Therefore models have been built in order to describe data and to make predictions. All models use probabilities for simulating the fragmentation rather than amplitudes. These Monte Carlo models use iterative procedures for the branchings such as string $\rightarrow$ string + hadron, or cluster $\rightarrow$ hadron, cluster $\rightarrow$ hadron + hadron.

One of the first popular approaches was the independent fragmentation by Field and Feynman [78]. The fragmentation of the partons generated in the hard scattering to hadrons proceeds independently: a quark q carrying a certain energy combines with an anti-quark $\bar{q}_{1}$ to form a meson $q \bar{q}_{1}$. This anti-quark $\bar{q}_{1}$ stems from a quark anti-quark pair $q_{1} \bar{q}_{1}$ formed out of the vacuum. The meson $q \bar{q}_{1}$ gets an energy fraction $z$ of the initial quark with the probability $f(z)$ (Field-Feynman fragmentation function),

$$
f(z)=1-a+3 a(1-z)^{2}, \text { with } a=0.77
$$

(Figure 2.5). This leaves an energy fraction $1-z$ to the remainder quark, which can pick another anti-quark $\bar{q}_{2}$ from the vacuum. While successful at the beginning, and besides conceptual difficulties (gluon fragmentation, energy and momentum conservation, Lorentz invariance), experiments at PEP and PETRA ruled the independent fragmentation out with the measurements of the particle flow between jets (string effect).

Today the best descriptions so far are obtained by models using the string or cluster fragmentation (Figure 2.6), which are discussed in the following sections.

\section{String Fragmentation}

The string picture used in the JETSET model is motivated by QCD confinement. Partons are not free at large distance. Partons, produced in the hard scattering process, are connected by a colour flux tube, which is stretched between quark and anti-quark. The transverse dimension of the tube is of the size of hadrons $\mathcal{O}(1 \mathrm{fm})$. The flux tube (string) has a linearly rising potential $V(r)=\kappa r$, leading to confinement. The string tension $\kappa$ is approximately $1 \mathrm{GeV} / \mathrm{fm}$. As for independent fragmentation from the vacuum a new massless quark anti-quark pair is formed, which breaks the string. In the case of gluon emission, which may be regarded as a kink in a string, the flux tube is interpreted as a string, that spans from the quark to the gluon, and from the gluon to the anti-quark. Therefore, in this model, string tension for the gluon is twice as large as for the quark. This corresponds to the expectation from QCD (and assuming LLA) for an infinite number of colours, while for three colours a factor $9 / 4$ is obtained: the relative contributions from the gluon bremsstrahlung off a gluon and a quark can be estimated using the Altarelli-Parisi 


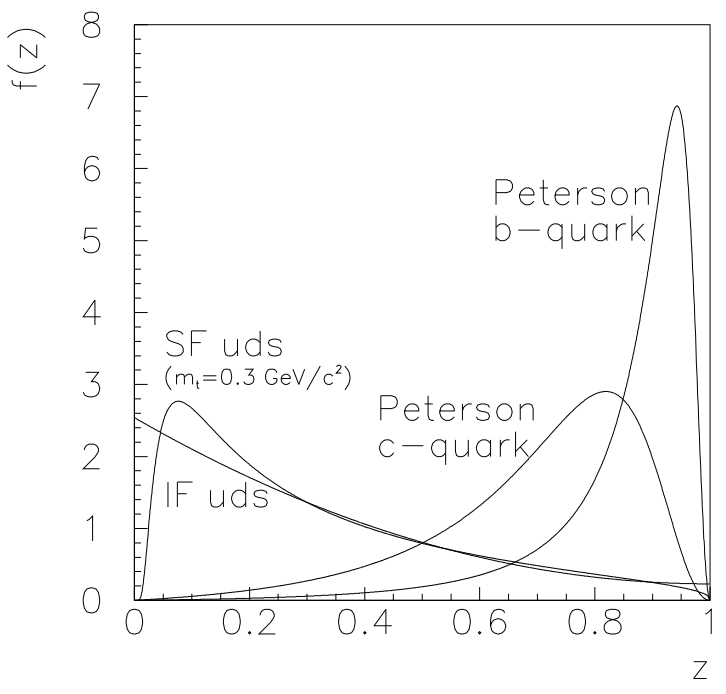

Figure 2.5: Comparison of fragmentation functions of independent fragmentation (IF), string fragmentation (SF) with transverse mass $m_{t}=\sqrt{m^{2}+p_{t}^{2}}=0.3 \mathrm{GeV} / c^{2}$, and Peterson fragmentation for the heavy flavours using $\epsilon_{c}=0.040$ and $\epsilon_{b}=0.0035$. For more details, see the description in the text.

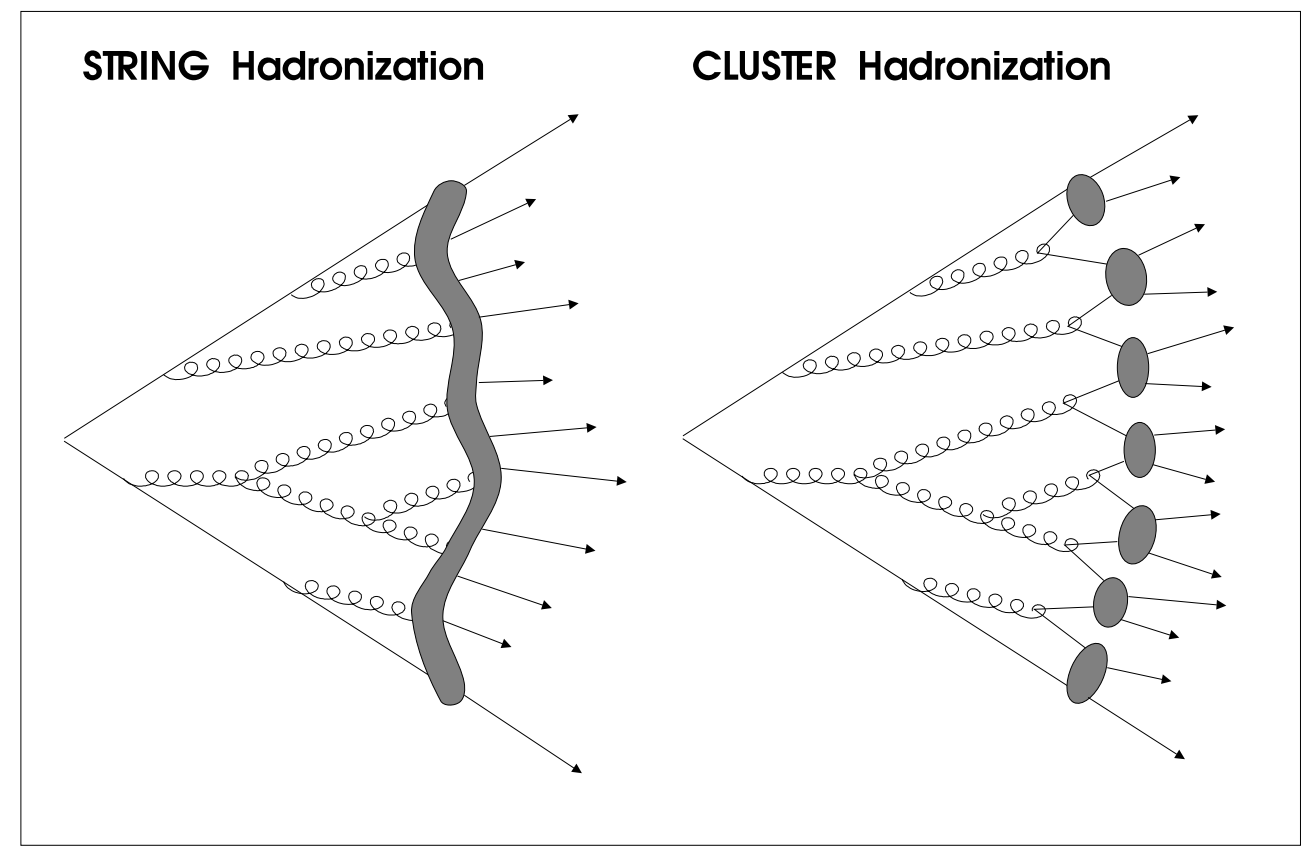

Figure 2.6: Illustration of the string and cluster fragmentation [64]. 
equation (see Section 2.2), where

$$
\frac{N_{c}}{C_{F}}=\frac{2}{1-1 / N_{c}^{2}}
$$

is the ratio close to the poles of its kernels.

Quarks have finite mass. A new $q \bar{q}$ pair created from the vacuum gains its energy from the potential energy of the string. This implies that the quarks must travel a distance to materialize, which depends on their mass (tunneling). Thus, the quark anti-quark pair production in the string is suppressed by the factor

$$
\exp \left(\frac{\pi}{\kappa}\left(-m_{\mathrm{q}}^{2}-p_{t}^{2}\right)\right)
$$

giving the tunneling probability for the new pair to appear. In addition to the quark mass $m_{\mathrm{q}}$, the new quark obtains a transverse momentum $p_{t}$. This is assumed to be Gaussian distributed with width $\sigma_{t}$ and flavour independent.

From the tunneling probability it follows naturally that heavy flavour (charm and bottom) production in the fragmentation is negligible. The production of strange quark pairs $s \bar{s}$ is suppressed by a factor $\approx 0.3$, which is due to the higher mass of the s-quark as compared to $\mathrm{u}^{-}$and $\mathrm{d}$-quark; usually, this strangeness suppression factor $\gamma_{\mathrm{s}}$ is a free parameter in the Monte Carlo model. More choices have to be made at this point: one would expect the ratio of vector meson to pseudoscalar meson production to be determined by the number of spin states, which is $3: 1$; differences in mass may change this number. From the average transverse momentum $\sigma_{t}$ discussed before and the length of the string pieces $\mathcal{O}(0.25 \mathrm{fm})$ one expects a smaller number. The longitudinal momentum distribution is mainly determined by the fragmentation function. The breakups of the string are causally independent, which restricts fragmentation functions. Instead of the original Field-Feynman fragmentation the Lund fragmentation

$$
f(z)=z^{-1}(1-z)^{a} \exp \left(-b m_{t}^{2} / z\right), z=\left(E+p_{l}\right)_{\text {hadron }} /\left(E+p_{l}\right)_{\text {quark }}
$$

is chosen for light flavours, u, d, and s. For charm and bottom quarks the Peterson fragmentation function

$$
f(z)=\left(z\left(1-\frac{1}{z}-\frac{\epsilon_{q}}{1-z}\right)^{2}\right)^{-1}, \text { with } \epsilon_{q} \sim \frac{1}{m_{q}^{2}}
$$

was found to be in better agreement with the data.

Baryons result when the string breakups generate diquark anti-diquark pairs. This simple picture, which always produces adjacent baryon anti-baryon pairs, needs some refinements to explain observations in experiment: the 'popcorn' mechanism allows for production of mesons between two baryons.

The principle of the popcorn mechanism is illustrated with space-time diagrams [62] in Figure 2.7. In the popcorn picture baryons are not directly produced, but rather as a correlated production of quark anti-quark pairs with right or wrong colour. In Figure 2.7a, between the primary quark pair, say of colour $r$ and $\bar{r}$, a new pair of the same colour 
is created, screens the colour field, and is pulled to the primary quarks forming mesons. If the new pair had the wrong colour, it would not screen the colour field of the primary quark and be just a quantum fluctuation and disappear (b). But when a second pair of the right colour appears (c), two baryons are formed. If two pairs are created between the pair of (b), a meson pops out between two baryons $(d)$.
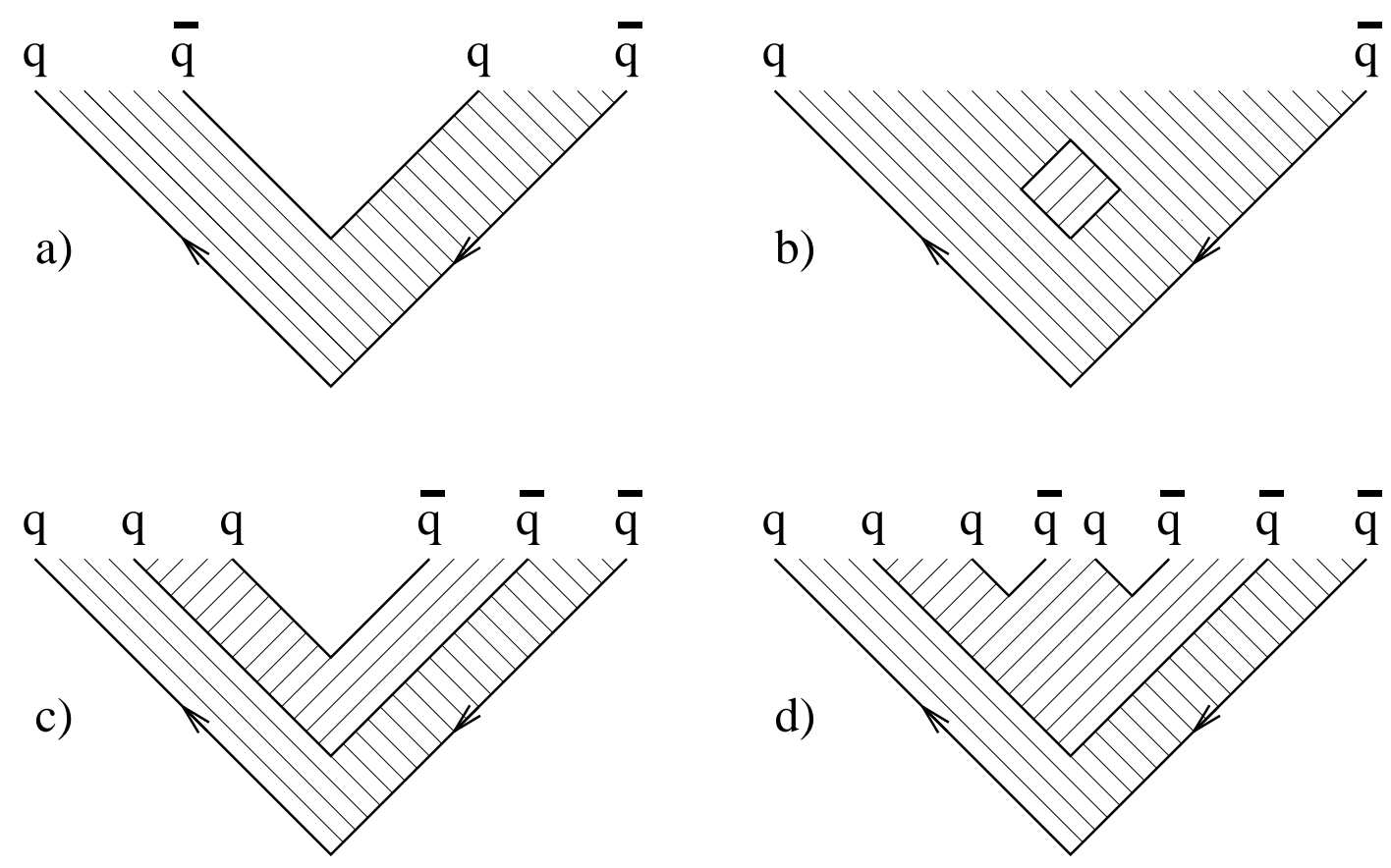

Figure 2.7: Space-time diagrams illustrating baryon production in the string model:

a) A new quark pair with the right colour, screening the colour field, is created and mesons are formed. b) A non-screening quark pair (wrong colour) appears and annihilates. c) Two quark pairs, a non-screening and a screening pair (diquark), are produced and form baryons. d) Inside the wrong colour quark pair two quark pairs are generated. This results in a meson being produced between the baryons.

\section{Cluster Fragmentation}

The cluster fragmentation (HERWIG) is based on the modified leading logarithmic approximation and local hadron parton duality. The model automatically includes coherence effects from the choice made in the parton shower evolution, such as gluon coherence and angular ordering. It is simpler, and has fewer parameters than JETSET. At the end of the parton shower, gluons are forced to decay to a quark anti-quark pair (or diquarks), where only u $\bar{u}$ and $d \bar{d}$ are allowed. Then quark anti-quark pairs with appropriate colour, which are closest in momentum space, are joined to clusters. These clusters are characterized by their mass, momentum, and flavour. The cluster mass distribution at the scale $Q_{0}^{2}$ is essentially independent of the momentum transfer of the electron-positron collision, i.e. 
the centre-of-mass energy (preconfinement). Mostly a cluster decays to two hadrons. For small masses, just one hadron may be formed. For high masses a cluster first splits into two clusters, after a new $u \bar{u}$, d $\bar{d}$, or $s \bar{s}$ pair was created.

In most cases, the cluster decays to two hadrons; all five available flavours are allowed for the additional quark and anti-quark. The decay is isotropic and determined by spin and phase space

$$
\sim\left(2 s_{1}+1\right)\left(2 s_{2}+1\right) 2 p^{*} / m .
$$

Here, $2 s_{1}$ and $2 s_{2}$ are the spins of the daughters, $p^{*}$ their momentum in the centre-of-mass system of the cluster, and $m$ the mass of the cluster.

\section{Particle Content and Particle Decays}

The generators take into account the production and decays of hadrons of the lightest multiplets. For baryons, this includes the spin- $1 / 2$ octet and spin-3/2 decuplet. Mesons with orbital angular momentum $L=0$, i.e. pseudoscalar and vector mesons are always produced. States with orbital angular momentum $L=1$ can be included. Some excited states, such as $\psi^{\prime}$ are also simulated. The masses of resonances are produced distributed according to a non-relativistic Breit-Wigner function, in a range typically $\pm 2 \Gamma$ around their known masses, where $\Gamma$ is the width of the Breit-Wigner distribution. The branching ratios of the decays are taken from the review of particle properties [79]. Some decays, not yet measured, are added using symmetry arguments. All decays are isotropic in their rest frame. For Dalitz decays and weak decays additional matrix elements are included. Polarization, which may be important for hadrons including the primary quarks, is not simulated.

Differences between the models exist. In JETSET several parameters control the decays and ensure isospin conservation. In HERWIG, where decays are determined by phase space and spin counting, a meson is selected from all available hadrons with the same flavour composition.

\subsection{Simpler Models}

\subsubsection{MLLA}

The production of a multihadronic event starts with the production of a quark anti-quark pair. Sometimes one or even several hard gluons are radiated off. The observation that the hadrons are collimated in jets indicated a duality between the parton and hadron level. It can be further postulated that this duality is local, i.e. the cross section on hadron level are proportional to those on parton level [80]. In connection with the modified leading logarithmic approximations (MLLA) [81], this local parton hadron duality (LPHD) allows predictions in the hadronization process. (Some considerations in this section include the next-to-leading approximation.)

The MLLA, which is also used in fragmentation models, can be tested by measuring inclusive particle spectra. A feature of the coherent parton emission is angular ordering: 
the later the gluon emission in the shower the smaller is its emission angle. As a consequence of this coherent QCD radiation low momentum partons are suppressed and so are the hadrons. In the observable $\xi=\ln \left(1 / x_{p}\right)=\ln (\sqrt{s} / 2 p)$ the differential cross section shows a maximum at $\xi=\xi^{*}$.

\section{Dependence on Centre-of-Mass Energy}

The MLLA combined with the LPHD predicts for the hadron spectrum [64, 81]

$$
\frac{1}{\sigma} \frac{\mathrm{d} \sigma}{\mathrm{d} \xi}=K_{\mathrm{LPHD}} \cdot f_{\mathrm{MLLA}}\left(\xi, E_{c m} / \Lambda_{e f f}\right)
$$

The cross section at hadron level is linked to the cross section at parton level $f_{\text {MLLA }}$ by $K_{\mathrm{LPHD}}$, which is essentially a constant, but depends on the hadron produced. It is responsible for the different yield for different particles. It includes the quantum numbers of the hadron, and therefore accounts for effects such as the strangeness suppression.

The dependence on $\xi$ is solely contained in $f_{\text {MLLA }}$, which can be calculated on parton level; the mean $\bar{\xi}$ (which is close to the position $\xi^{*}$ of the maximum; see below) of the $\xi$ distribution can be calculated. Its $E_{c m}$ dependence is given by

$$
\begin{gathered}
\bar{\xi}=\frac{1}{2} \tau\left(1+\frac{\rho}{24} \sqrt{\frac{48}{\beta \tau}}\right)+\mathcal{O}(1), \\
\text { with } \tau=\ln \frac{E_{c m}}{2 \Lambda_{e f f}} \text {, and } \rho=11+2 N_{f} / N_{c}^{3}, \beta=11-2 N_{f} / C_{A} .
\end{gathered}
$$

The number of colours and the colour factor are given by $N_{c}=C_{A}=3$. While one could expect that the number of flavours $N_{f}$ is five, in the fragmentation only the light flavours contribute and $N_{f}=3$ is used. The result becomes

$$
\bar{\xi}=\frac{1}{2} \ln \frac{E_{c m}}{2 \Lambda_{e f f}}+\frac{101}{324} \sqrt{3} \sqrt{\ln \frac{E_{c m}}{2 \Lambda_{e f f}}},
$$

where $\Lambda_{e f f}$ is the only free parameter. It is a cutoff parameter on parton energies and is related to the hadron mass, with heavier particles having a harder spectrum.

A simplified version is proposed in [80] for coherent parton shower evolution including angular ordering, and for an incoherent parton shower. The differential hadron cross sections can be expressed by the formula [64]

$$
\frac{\mathrm{d} \sigma}{\mathrm{d} \xi}=1+\alpha_{\mathrm{s}} \xi\left(\ln \frac{E_{c m}}{2 \Lambda_{e f f}}-\frac{\xi}{k}\right) .
$$

The parameter $k$ distinguishes between the coherent $(k=1)$ and the incoherent $(k=2)$ model.

The maximum position $\xi^{*}$ of the distribution as function of the centre-of-mass energy $E_{c m}$ is then given by

$$
\xi^{*}=\frac{k}{2} \ln \frac{E_{c m}}{2 \Lambda_{e f f}}
$$


with a slope

$$
S=\frac{\mathrm{d} \xi^{*}}{\mathrm{~d} \ln E_{c m}}=\frac{k}{2} .
$$

The determination of this slope tests, whether a shower is coherent or not.

\section{Shape of the Particle Spectra}

The formulas evaluated with MLLA are not well suited for numerical calculations and for the empirical comparison with momentum spectra of identified particles. It is convenient, however, to expand the differential cross section around its mean $\bar{\xi}$ in the quantity

$$
\delta=\frac{\xi-\bar{\xi}}{\sigma}
$$

with width $\sigma$, skewness $s$ and kurtosis $k$. The latter are defined through the central moments $\mu_{n}$ of the $\xi$ distribution, which are the expectation values $\mu_{n}=E\left((\xi-\bar{\xi})^{n}\right)$ :

$$
\sigma=\sqrt{\mu_{2}}, \quad s=\mu_{3} / \sigma^{3}, \quad k=\mu_{4} / \sigma^{4}-3 .
$$

With an additional normalization factor $K$, the expansion is given by

$$
\frac{1}{\sigma} \frac{\mathrm{d} \sigma}{\mathrm{d} \xi}(\xi ; \bar{\xi}, \sigma, s, k, K)=\frac{K}{\sigma \sqrt{2 \pi}} \exp \left(\frac{1}{8} k-\frac{1}{2} s \delta-\frac{1}{4}(2+k) \delta^{2}+\frac{1}{6} s \delta^{3}+\frac{1}{24} k \delta^{4}\right) .
$$

The parameters mean, width, skewness, and kurtosis can be calculated in leading and next-to-leading order. The shape is almost Gaussian in the vicinity of the maximum; the peak position $\xi^{*}$ is very close to the mean $\bar{\xi}: \xi^{*}-\bar{\xi} \approx 101 / 288 \approx 0.351$ for three light flavours in the fragmentation. Often, just a Gaussian function is used for the determination of the maximum position; in Ref.[64] a Gaussian shape is combined with the powerlaw behaviour of the fragmentation function for high momenta: $\mathrm{d} \sigma / \mathrm{d} x \sim x^{a}(1-x)^{b} \exp \left(-c \ln (x)^{2}\right)$. In the LPHD, which locally relates partons to hadrons, decays are another form of fragmentation and should not shift the maximum position.

\subsubsection{Spin-Counting, Isospin}

Another, but purely phenomenological approach for describing the rates of identified particles is discussed in Ref. [82]. It is motived by the fact that the tuning of the parameters available in fragmentation models may allow to describe the data; their large number, however, may hide the physics and regularities behind, such as spin degeneracy. Baryon production rates as a function of the particle mass squared are found to lie on one universal curve, when spin $J$ and isospin are taken into account. Usually the rate for each isospin state is taken separately. Taking all isospin states together the general mass dependence ( $M$ is the hadron mass) is described by the phenomenological formula

$$
<n>=\frac{2 J+1}{2 I_{m}+1} a \exp \left(-b M^{2}\right)
$$


with only two free parameters $(a, b) .\langle n\rangle$ denotes the average multiplicity for a particle (not counting anti-particles).

In this paper a modified isospin $I_{m}$ is introduced for mesons. The four kaons $\left(\mathrm{K}^{+}, \mathrm{K}^{0}\right.$, $\left.\overline{\mathrm{K}}^{0}, \mathrm{~K}^{-}\right)$have $I_{m}=3 / 2$, the three pions $\left(\pi^{+}, \pi^{0}, \pi^{-}\right)$have $I_{m}=1$, and the two etas $(\eta$, $\eta^{\prime}$ ) have $I_{m}=1 / 2$. Corresponding rules are set up for the vector mesons.

With these assumptions a good description of the data measured at LEP can be fitted with the phenomenological formula. Data at lower energies (PEP and PETRA) are described with the same slope parameter $b$.

\subsubsection{Thermodynamical Model}

In a recent paper [83], hadronization in $\mathrm{e}^{+} \mathrm{e}^{-}$annihilation is treated in a thermodynamical approach. Most events are 2-jet events with each of the two jets treated as a hadron gas in thermodynamical and chemical equilibrium before freeze-out, the decoupling of the hadrons which then decay. The decay tables are similar to JETSET and taken from Ref. [79]. The model is characterised by the temperature; the volume; a parameter $\gamma_{\mathrm{s}}$ describing the partial strangeness chemical equilibrium; a symmetry group U $(1)^{4}$ corresponding to the conservation of baryon number, strangeness, charm, and beauty. With the assumption that additional jets have the same temperature, the 2-jet approach should be a good approximation for the relative rates of particle production.

In practice the two jets cannot be independent because a quark has colour, fractional charge and a baryon number. Two different schemes have been tried. (i) An uncorrelated jet scheme where strangeness, charm, and bottom of the parent quark are conserved, while the baryon number is assumed to vanish. (ii) A correlated jet scheme where allowance

for baryon number and $s \bar{s}$ sharing between the two jets is made. The two jets may have opposite baryon number (up to 1) and opposite strangeness (up to 2).

The model is tuned separately to PEP/PETRA data and to LEP data. 


\section{Chapter 3}

\section{Data Taking}

\subsection{LEP Collider}

The large electron-positron collider LEP [79, 84] is located at the French-Swiss border between Geneva and the Jura mountains. It is a ring of $27 \mathrm{~km}$ circumference, about $100 \mathrm{~m}$ underground. The tunnel is not horizontal but inclined by $1.42 \%$ for geological reasons. The electron and positron beams are kept at their orbit by 3304 dipole magnets arranged in eight bending sections. At energies at the $\mathrm{Z}$ resonance, i.e., $46 \mathrm{GeV}$ beam energy, a field of $0.48 \mathrm{~T}$ is required. On two straight sections radiofrequency cavities are installed. They serve to increase the lepton energy from $20 \mathrm{GeV}$ at injection to $46 \mathrm{GeV}$. During physics running at stable beams, the cavities have to provide the energy which is lost by synchrotron radiation (130 MeV per turn).

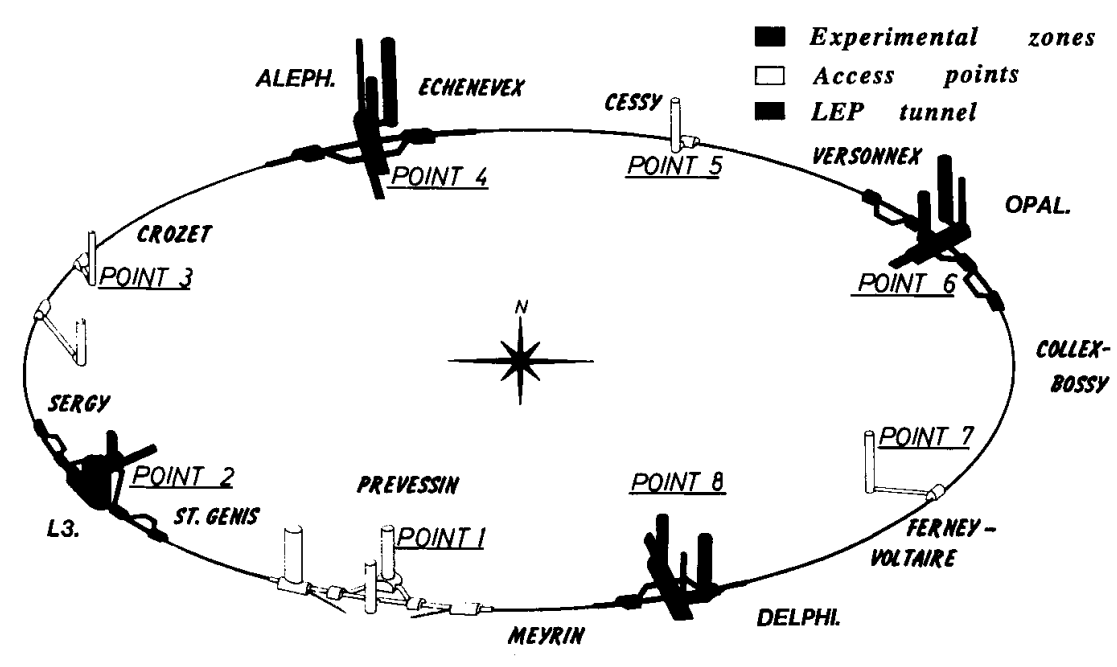

Figure 3.1: Sketch of the LEP storage ring showing the location of the four experimental zones. 
In Figure 3.1 a sketch of LEP with the location of the four experiments is given. The positrons circulate clockwise, the electrons counter-clockwise, the direction of the latter being chosen as $z$-direction in the experiments. The $y$-direction is vertical (upward), while the $x$-direction points to the centre of LEP, but is horizontal. At LEP startup the beams each consisted of four bunches so that there are eight possible collision points. Experiments are installed in points which were given even numbers. The bunches are then separated electrostatically at the odd numbered points to avoid interactions there. In this way the luminosity $\mathcal{L}$ is increased compared to the use of only two bunches per beam. One has

$$
\mathcal{L}=f_{r e v} k \frac{N^{+} N^{-}}{4 \pi \sigma_{x} \sigma_{y}} .
$$

Here, $f_{\text {rev }}$ is the LEP revolution frequency, $k$ is the number of bunches (4 or 8 ), $N^{+}$and $N^{-}$are the number of positrons and electrons per bunch $\left(\approx 4 \cdot 10^{11}\right)$, and $\sigma_{x} \approx 200 \mu \mathrm{m}$ $\left(\sigma_{y} \approx 8 \mu \mathrm{m}\right)$ is the horizontal dimension of the beam in $x-(y-)$ direction.

The luminosity of LEP is typically $10^{30} \mathrm{~cm}^{-2} \mathrm{~s}^{-1}$ to few times $10^{31} \mathrm{~cm}^{-2} \mathrm{~s}^{-1}$. Around 10000 hadronic events per day are produced at the $\mathrm{Z}$ pole energy $\left(N=\int \mathcal{L} \sigma_{\text {had }} \mathrm{d} t\right)$. The numbers of hadronic $Z$ decays, which were used in the electro-weak working group of the four LEP experiments are listed in Table 3.1 [68] together with the time-integrated luminosity seen by each experiment [85], which is displayed in Figure 3.2.

\begin{tabular}{|c|c|c|r|r|r|c|}
\hline & ALEPH & DELPHI & L3 & OPAL & LEP & Lumi.(pb $\left.{ }^{-1}\right)$ \\
\hline \hline$' 90-' 91$ & 451 & 356 & 416 & 454 & 1677 & 24.9 \\
$' 92$ & 680 & 697 & 678 & 733 & 2788 & 28.6 \\
'93 & 640 & 677 & 654 & 646 & 2617 & 40.0 \\
'94 & 1281 & 1144 & 1362 & 1524 & 5311 & 64.4 \\
'95 & & & & & & 46.1 \\
\hline total & 3052 & 2874 & 3110 & 3357 & 12393 & 204.0 \\
\hline
\end{tabular}

Table 3.1: The LEP statistics of hadronic events used for the Z line shape analysis (in units of $10^{3}$ events) [68] and the integrated luminosity seen by each experiment (in $\mathrm{pb}^{-1}$ ) $[85]$.

The high event statistics makes this largest collider a unique machine. An accurate energy calibration of the beam energy, however, is mandatory [86] in addition; the primary goals of the LEP program, the measurement of the $\mathrm{Z}$ mass and width, require a high precision on the energy determination. The LEP beam energy is defined by the magnetic field of the bending magnets. The field is monitored by a reference magnet in series with the bending magnets. Corrections for temperature and ageing are done. Another possibility is the calibration comparing the rotation frequency of $20 \mathrm{GeV}$ protons and positrons. The extrapolation to the $Z$ energy, however, reduces the accuracy.

The most precise calibration up to now uses the method of resonant depolarization. The electrons and positrons of the beam are transversely polarized through the emission 


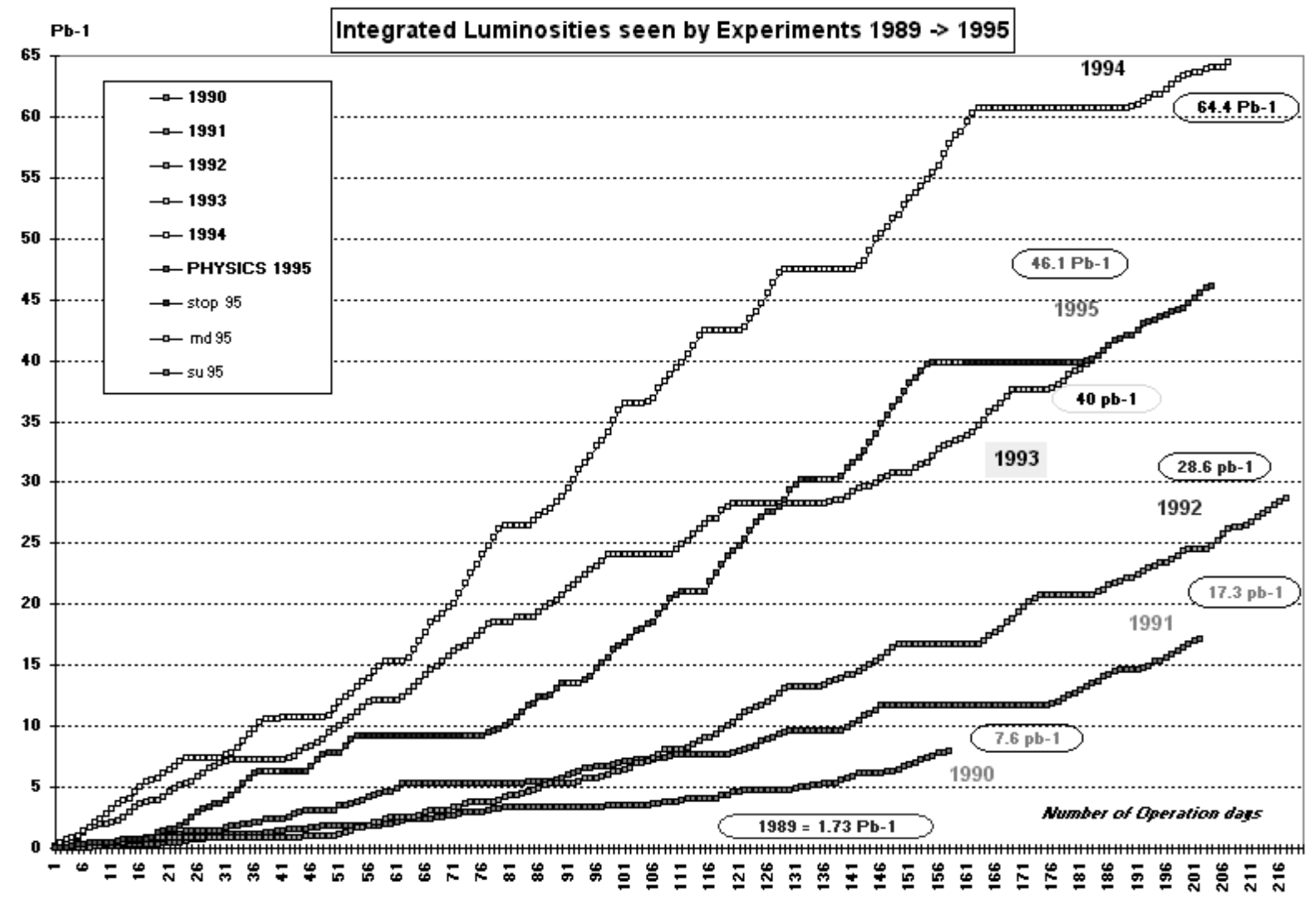

Figure 3.2: Integrated luminosity seen by the LEP experiments, 1989-1995 [85].

of synchrotron radiation. The Compton back-scattered photons of right- and left-handed circularly polarized laser beams from the circulating beam are measured with an up-down asymmetry in an electromagnetic calorimeter.

With all these calibrations, and including corrections such as for the tidal effect, the total uncertainty of the beam energy translates in an energy uncertainty of the $\mathrm{Z}$ mass of $1.5 \mathrm{MeV} / \mathrm{c}^{2}[68]$.

\subsection{Detectors}

At four of the eight straight sections four collaborations have installed their detectors: ALEPH [87], DELPHI [88], L3 [89], and OPAL [90]. The design of the detectors is guided by the physics of interest. The detectors consist of several subdetectors each dedicated to special aspects of the final state under investigation.

The main physics goal at LEP is the test of the Standard Model. The mass and width of the $\mathrm{Z}$ boson are being measured to a high precision. The couplings of the leptons and quarks to $\gamma / Z$ are investigated. Special emphasis is put on the study of $\tau$-decays. The $\tau$-polarization gives a good insight into the couplings. The high production probability 
of the heavy flavours, charm and bottom, allows for investigations of effects, such as branching ratios, hadron masses, time dependent mixing etc. Indirect information on the top mass is extracted and the influence from the Higgs mass is studied. Direct Higgssearch is one of the most important topics in the new physics area. Supersymmetric particles, if they exist in the accessible range, should not be able to escape detection. The strong interaction, with confinement and asymptotic freedom still not understood, is to be investigated. The perturbative part (e.g, $\alpha_{\mathrm{s}}$-determination) and the non-perturbative part, fragmentation and particle production, guided the design of the detectors as well.

In addition, the general features of the detectors have to keep the systematic uncertainties for their measurements very small to profit from the excellent energy calibration of LEP and to efficiently use the high event statistics.

All LEP detectors have therefore in common, a good hermiticity as well as a good efficiency. The total (hadronic) energy has to be measured as completely as possible. The total absorption guaranties that all particles except neutrinos are seen. Muons also deposit only a small fraction of their energy, but are detected in special muon chambers and by their characteristic signature in the hadron calorimeter. Care for efficient detection and identification of leptons is taken. In general particle identification is provided. Good two-track resolution is possible inside jets of hadrons; energy loss measurements on more than hundred samplings, high granularity of the calorimeters are needed. High precision tracking and vertexing of secondary vertices guaranties good detection and momentum resolution for charged particles, even in the case when they do not come from the primary interaction point.

The trigger system ensures that all events of interest are seen with low background. The triggers of the four LEP detectors have a high redundancy. For example, hadronic events are found when the energy exceeds a few $\mathrm{GeV}$ in the electromagnetic calorimeter (total energy trigger), or two tracks are seen together with energy deposition in the hadron calorimeter, which exceeds the energy expected for a minimum ionizing particle ( $\mu$-trigger). The efficiency for hadronic events is $\geq 99.99 \%$ with an uncertainty of $0.01 \%$.

These requirements lead to four LEP detector designs with a similar general outline, while the detectors differ in their details (see Table 3.2, [91]). The detectors show a cylindrical symmetry around the beam pipe. In the forward direction, calorimeters are installed for the measurement of the luminosity with high precision. The main body has closest to the beam pipe a vertex detector mounted, with precision measurements of the hits from tracks crossing; a general tracking system, which may consist of separate tracking devices; an electromagnetic calorimeter for measuring electrons and photons; a coil of a magnet in order to bend charged particles for the momentum measurement in the tracking devices; a hadron calorimeter for hadronic showers absorbing strong interacting particle, but passed by muons; the latter are detected in the muon chambers, surrounding the experiments.

In the following all four detectors will be described. The ALEPH detector will be presented in some detail. For the other three detectors, special aspects relevant for the subject of this paper are discussed. 


\begin{tabular}{|c|c|c|c|c|}
\hline B-field & $\begin{array}{c}\text { ALEPH } \\
1.5 \mathrm{~T}\end{array}$ & $\begin{array}{c}\text { DELPHI } \\
1.2 \mathrm{~T}\end{array}$ & $\begin{array}{c}\mathrm{L3} \\
0.5 \mathrm{~T}\end{array}$ & $\begin{array}{l}\text { OPAL } \\
0.435 \mathrm{~T}\end{array}$ \\
\hline $\begin{array}{l}\text { Si VTX } \\
\mathrm{r}=0.1 \mathrm{~m}\end{array}$ & $\begin{array}{c}2 \text { layers } \\
R \phi z \\
12 \mu, 12 \mu\end{array}$ & $\begin{array}{c}3 \text { layers } \\
R \phi z \\
9 \mu, 7.6 \mu\end{array}$ & $\begin{array}{c}2 \text { layers } \\
R \phi z\end{array}$ & $\begin{array}{c}2 \text { layers } \\
R \phi z \\
5 \mu, 13 \mu\end{array}$ \\
\hline $\begin{array}{l}\text { inner tr. } \\
\mathrm{r}=0.3 \mathrm{~m}\end{array}$ & $\begin{array}{c}8 \mathrm{pts}, 150 \mu, 5 \mathrm{~cm} \\
\text { drift ch. }\end{array}$ & $\begin{array}{l}24 p t s, 100 \mu \\
\text { jet ch. } R \phi\end{array}$ & \multirow[b]{2}{*}{37 pts, 30 to $70 \mu$} & \multirow{4}{*}{$\begin{array}{c}159 \mathrm{pts} \\
135 \mu, 6 \mathrm{~cm} \\
\text { JET } 4 \text { atm }\end{array}$} \\
\hline \multirow{2}{*}{$\begin{array}{l}\text { main tr. } \\
\text { detector } \\
\mathrm{d} E / \mathrm{d} x\end{array}$} & \multirow{3}{*}{$\begin{array}{c}\text { TPC, } 1 \mathrm{~atm} \\
4.6 \%\end{array}$} & TPC, 1atm & & \\
\hline & & $5.5 \%$ & \multirow{2}{*}{$\begin{array}{c}\text { BGO e-m cal } \\
4 \% \text { at } 200 \mathrm{MeV} \\
\text { HCAL } 60 \mathrm{U} \text { plates } \\
55 \% / \sqrt{E}\end{array}$} & \\
\hline $\mathrm{r}=1.1 \mathrm{~m}$ & & $\begin{array}{c}\mathrm{RICH}, 1 \mathrm{cmC}_{6} \mathrm{~F}_{14} \\
\text { gas } \mathrm{C}_{5} \mathrm{~F}_{12}\end{array}$ & & \\
\hline \multirow[t]{2}{*}{$\mathrm{r}=1.8$} & \multirow{2}{*}{$\begin{array}{l}\text { ECAL } 21.5 X_{0} \\
18 \% / \sqrt{E}, 3 \mathrm{sp}\end{array}$} & OD 5pts, $150 \mu$ & \multirow{2}{*}{$\begin{array}{l}\text { filter } 1 \lambda, 5 \text { pts } \\
\text { support pipe }\end{array}$} & \multirow{2}{*}{$\begin{array}{c}z \operatorname{chb} 6 \times 300 \mu \\
\text { coil } 1.7 X_{0}\end{array}$} \\
\hline & & \multirow{2}{*}{$\begin{array}{l}\mathrm{HPC} 18 \mathrm{X}_{0} \\
33 \% / \sqrt{E}, 9 \mathrm{sp} \\
\text { coil } 2 \mathrm{X}_{0} \\
\end{array}$} & & \\
\hline $\mathrm{r}=2.2$ & coil $1.6 \mathrm{X}_{0}$ & & \multirow{4}{*}{$\begin{array}{c}\text { muon chb } \\
\text { 3sets } \\
\text { lever arm } 2.7 \mathrm{~m}\end{array}$} & $\begin{array}{c}\text { lead glass } 20 \mathrm{X}_{0} \\
5 \% / \sqrt{E}\end{array}$ \\
\hline $\mathrm{r}=2.9$ & HCAL $1.2 \mathrm{mFe}$ & HCAL $1.2 \mathrm{mFe}$ & & HCAL $1 \mathrm{mFe}$ \\
\hline \multirow[b]{2}{*}{$\mathrm{r}=5.7$} & \multirow{2}{*}{$\begin{array}{c}\text { muon chb } \\
2 \text { layers } \\
\text { lever arm } 0.5 \mathrm{~m}\end{array}$} & $\begin{array}{l}\text { muon chb } \\
2 \text { layers }\end{array}$ & & \multirow{2}{*}{$\begin{array}{c}\text { muon chb } \\
4 \text { layers } \\
\text { lever arm } .7 \mathrm{~m}\end{array}$} \\
\hline & & lever arm $.3 / .6 \mathrm{~m}$ & & \\
\hline $\begin{array}{l}\text { Lumi. } \\
\text { forward }\end{array}$ & $\begin{array}{c}\text { calorimeter } \\
\text { tungsten/silicon } \\
24-58 \mathrm{mrad}\end{array}$ & $\begin{array}{l}\text { calorimeter } \\
\text { lead/scint. } \\
29-185 \mathrm{mrad}\end{array}$ & $\begin{array}{c}\text { wire ch. } \\
\text { BGO+prop. } \\
25-70 \text { mrad }\end{array}$ & $\begin{array}{l}\text { calorimeter } \\
\text { tube ch. } \\
58-120 \mathrm{mrad}\end{array}$ \\
\hline
\end{tabular}

Table 3.2: Characteristics of the four LEP experiments [87]-[91].

\section{ALEPH Detector}

The ALEPH detector (Figure 3.3) [87] shows the typical cylindrical symmetry around the beam pipe. The interaction point of the electron and positron beams is at the centre of the detector. The tracking chambers and the electromagnetic calorimeter are immersed in a solenoidal magnetic field of $1.5 \mathrm{~T}$ produced by the superconducting coil (with a length of $6.4 \mathrm{~m}$ and a diameter of $5.3 \mathrm{~m}$ ). Outside the coil the hadron calorimeter is used as return yoke.

The beam pipe inside ALEPH, with a length of $5.5 \mathrm{~m}$ extends between the two 'low- $\beta$ ' quadrupoles, which focuses the electron and positron beams onto the interaction point. The tube is made of $1.5 \mathrm{~mm}$ thick aluminium, with an inner diameter of $106 \mathrm{~mm}$. The central part ( $760 \mathrm{~mm}$ length), however, is made of beryllium, $1.1 \mathrm{~mm}$ thick.

Closest to the interaction point, the silicon vertex detector (VDET) is installed. It consists of two concentric rings with average radius $6.5 \mathrm{~cm}$ and $11.3 \mathrm{~cm}$. The inner layers has 9 silicon wafers in azimuth, the outer layer has 15 wafers; both layers are four wafers 


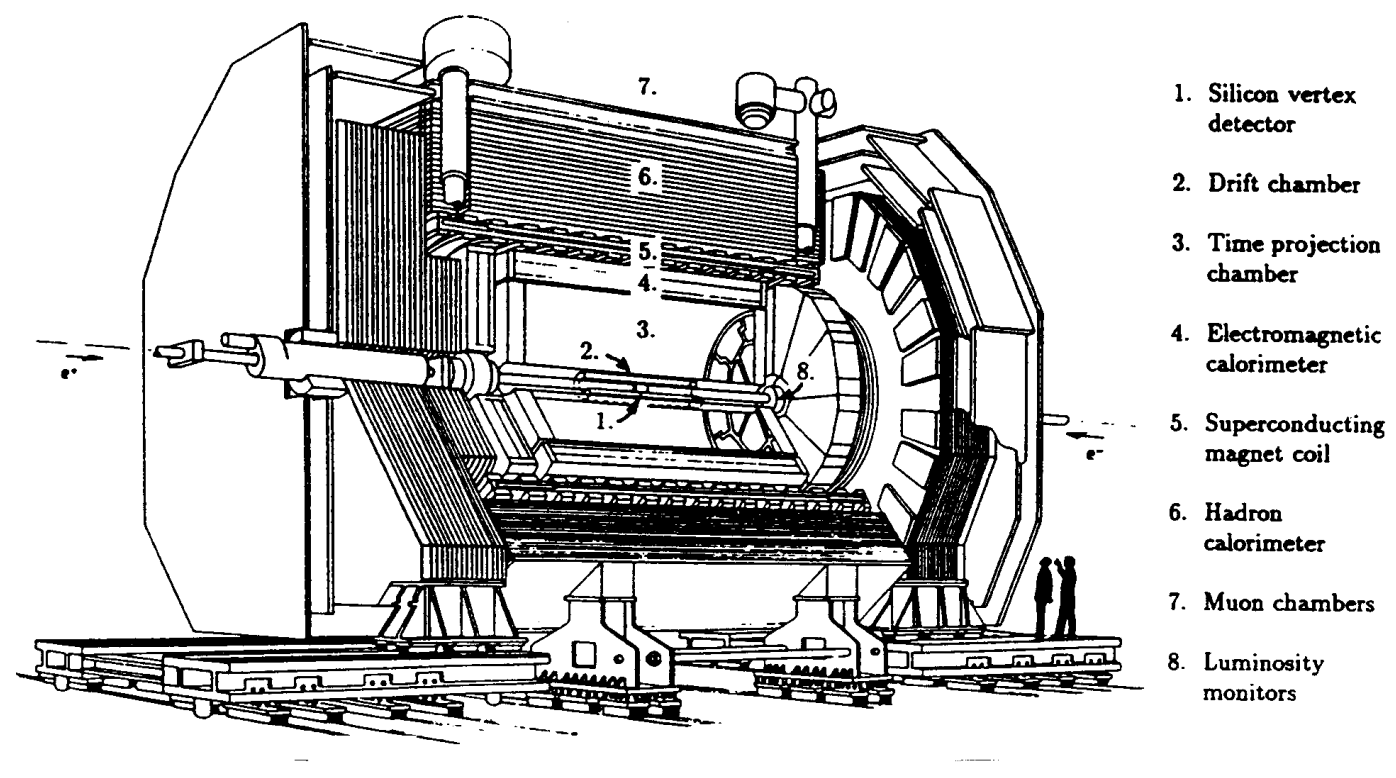

Figure 3.3: ALEPH detector [87].

$\left(5.12 \times 5.12 \times 0.03 \mathrm{~cm}^{3}\right)$ long in $z$-direction. The arrangement in azimuth is such that the wafers overlap by $5 \%$. This allows an internal relative alignment with tracks passing through adjacent wafers. The point resolution in the $r-\phi$ and $r-z$ view is $12 \mu \mathrm{m}$. The hit association of VDET hits to tracks extrapolated from the TPC is found by Monte Carlo to be $98 \%$ for tracks in hadronic events with two vertex hits in the acceptance of the vertex detector: $|\cos \theta|<0.85$.

Around the vertex detector the inner tracking chamber (ITC) is built with the same polar geometrical acceptance as the vertex detector. This conventional cylindrical drift chamber is filled with $80 \%$ argon and $20 \%$ carbon dioxide with ethanol. The chamber provides eight measurements in $r-\phi$ in a radial range between $16 \mathrm{~cm}$ and $26 \mathrm{~cm}$, with the wires stretched in z-direction and arranged in eight concentric layers of hexagonal drift cells. In $r-\phi$ the position of hits is measured to $150 \mu \mathrm{m}$; in $z$ the position is obtained by the measurements of the difference of the arrival time of the pulses at both ends of the $2 \mathrm{~m}$ long wires. The precision reached is $5 \mathrm{~cm}$. However, only the $r-\phi$ measurements are used for the tracking; the information of $z$ can be used for track association with the tracks reconstructed in the TPC. An important aspect of the ITC is that it is the only tracker used for the trigger.

The time projection chamber (TPC) serves as the main tracking chamber in ALEPH. In a volume extending in radius from $0.3 \mathrm{~m}$ to $1.8 \mathrm{~m}$, with a length of $4.4 \mathrm{~m}$ up to 21 space points are measured. The ionization charge is recorded in proportional wire chambers at both ends of the drift volume, reading out cathode pads arranged in 21 concentric circles; up to $338 \mathrm{~d} E / \mathrm{d} x$ samples are used for particle identification. The $z$ coordinate of the hits in the TPC is calculated from the drift time of the electrons collected. For this, the magnet field, electric field both pointing in horizontal direction (and their distortions), 
and the drift velocity must be known perfectly. These quantities are determined from a measured magnetic field map, by laser calibration and study of reconstructed tracks and their vertices. The resolution is found in $r-\phi$ as $173 \mu \mathrm{m}$ and in $z$ as $740 \mu \mathrm{m}$.

In hadronic events, $98.6 \%$ of the tracks are reconstructed, when they cross at least four out of 21 pad rows, $|\cos \theta|<0.966$. The momentum resolution has been determined with di-muon events. The transverse momentum resolution $\sigma\left(1 / p_{t}\right)$ is $1.2 \times 10^{-3}\left(p_{t}\right.$ in $\left.\mathrm{GeV} / c\right)$ for the TPC alone; including ITC an VDET the resolution is $\sigma\left(p_{t}\right) / p_{t}=0.0006 \cdot p_{t} \oplus 0.005$ $\left(p_{t}\right.$ in $\left.\mathrm{GeV} / c\right) ; \oplus$ implies that the two errors are added in quadrature.

The TPC is surrounded by the electromagnetic calorimeter (ECAL), which consists of a barrel part and two endcaps, in order to measure electromagnetic energy in an angular range $|\cos \theta|<0.98$. With its fine segmentation in projective towers of approximately $3 \mathrm{~cm}$ by $3 \mathrm{~cm}$, i.e. $0.9^{\circ}$ by $0.9^{\circ}$, the angular resolution is $\sigma_{\theta, \phi}=2.5 / \sqrt{E}+0.25(E$ in $\mathrm{GeV}$; $\sigma_{\theta, \phi}$ in mrad). The towers are read out in three segments in depth called storeys of 4,9 , and 9 radiation lengths. This lead-proportional tube chamber has an energy resolution for electromagnetic showers of $\sigma_{E} / E=0.18 / \sqrt{E}+0.009(E$ in $\mathrm{GeV})$.

The outer shell used as return yoke, is the hadron calorimeter (HCAL). It is made from iron plates of $5 \mathrm{~cm}$ thickness, interleaved with 22 layers of plastic streamer tubes and one layer of tubes in front. The towers are arranged in projective direction to the primary vertex with a solid angle of $3.7^{\circ}$ by $3.7^{\circ}$, corresponding to $4 \times 4$ of the electromagnetic calorimeter towers. Both the cathode pads defining the towers (pads of different tubes forming one tower are connected galvanically within one storey) and wires in the $1 \mathrm{~cm}$ wide tubes are read out. The latter are used for muon identification and as a trigger. The energy resolution can be parameterized $\sigma_{E} / E=0.85 / \sqrt{E}(E$ in $\mathrm{GeV})$. In addition, two double layers of streamer tubes are installed around the hadron calorimeter outside the magnetic field and serve as muon detectors.

\section{DELPHI Detector}

The layout of the DELPHI detector [88] is shown in Fig.3.4. The subdetectors are arranged in a cylinder symmetrical arrangement with only the hadron calorimeter and the muon chambers being outside the superconducting coil. The vertex detector closest to the beam pipe is made of silicon wafers. It provides measurements in three layers with information in both $z$ - and $r-\phi$-direction. The single hit resolution is found to be $9 \mu \mathrm{m}$ and $7.6 \mu \mathrm{m}$. The vertex detector is surrounded by the inner detector (ID) of a jet-chamber geometry with five multi wire proportional chambers (MWPC) layers. The main tracking device is a Time Projection Chamber (TPC) measuring up to 16 space points per track. Together with the outer detector (OD) with 5 layers of drift tubes the four tracking chambers provide a momentum resolution of $\sigma(p) / p=0.0006 \cdot p(p$ in $\mathrm{GeV} / \mathrm{c})$.

A specialty of the DELPHI detector is the Ring Imaging Cherenkov detector (RICH) enclosed by the outer detector. The particle identification in the RICH complements the identification with $\mathrm{d} E / \mathrm{d} x$ in the TPC. The DELPHI collaboration has chosen to use a gas and a liquid RICH $\left(\mathrm{C}_{5} \mathrm{~F}_{12}\right.$ and $\left.\mathrm{C}_{6} \mathrm{~F}_{14}\right)$, having two different refractive indices. While the $\mathrm{d} E / \mathrm{d} x$ measurement is most powerful in the momentum range below $1 \mathrm{GeV} / c$, the liquid radiator allows for particle identification from $0.7 \mathrm{GeV} / c$ to $8 \mathrm{GeV} / c$ and the 


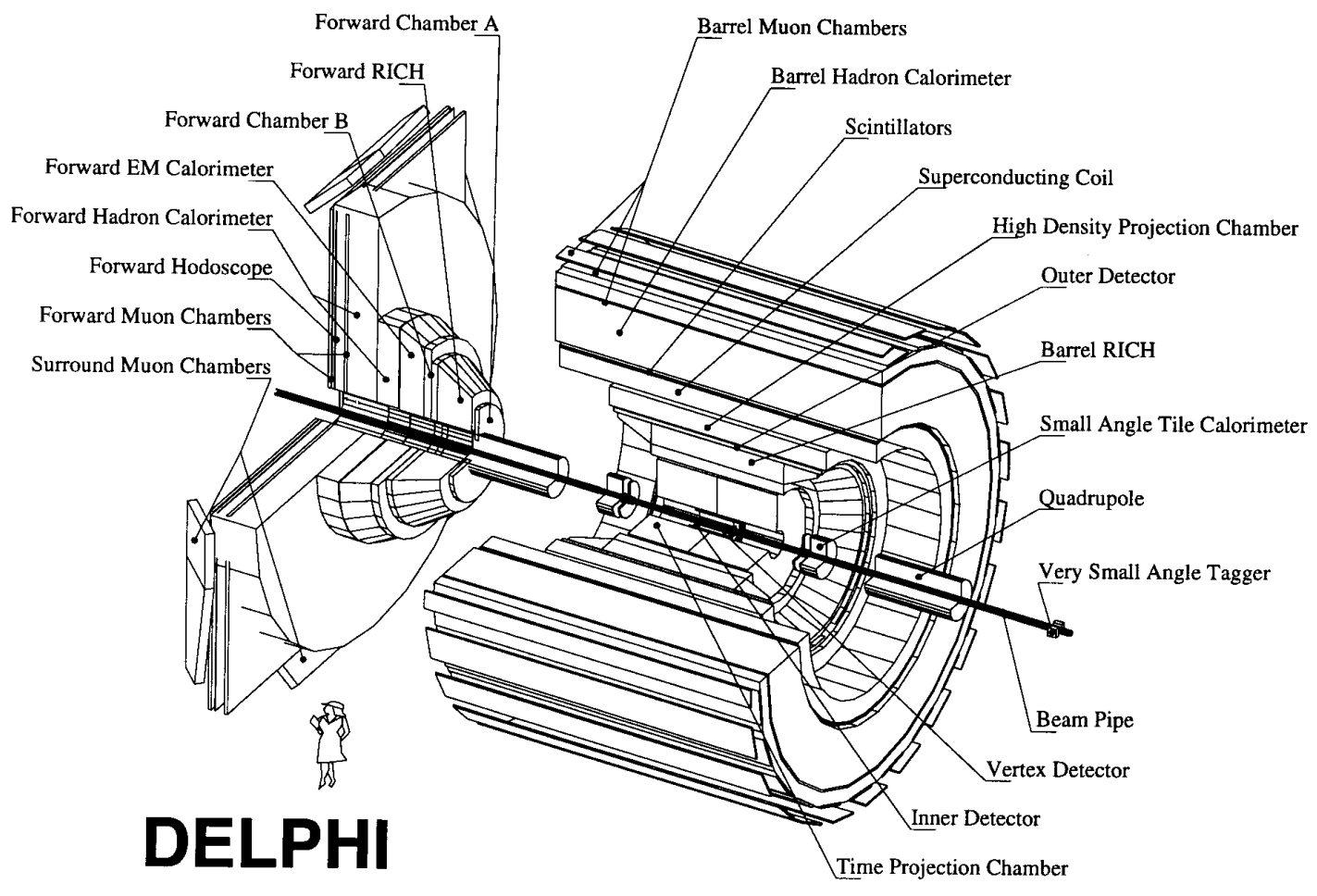

Figure 3.4: DELPHI detector [88].

gaseous radiator from $2.5 \mathrm{GeV} / c$ to $25 \mathrm{GeV} / c$, with angular resolution between $1.2 \mathrm{mrad}$ and $5.2 \mathrm{mrad}$. (See as well in the Chapter 4 on particle identification and Figure 4.1.)

The high density projection chamber (HPC) consists of layers of TPCs, which are separated by lead wires. These wires separate the drift cells and provide the drift field, but also serve as converter material for the electromagnetically interacting particles. The energy deposits on the pads are monitored with $\pi^{0}$ 's, where one decay photon converted in the material in front of the HPC and the momentum is precisely measured: with the $\pi^{0}$ mass as a constraint, the energy resolution is measured to $\sigma(E) / E=0.33 / \sqrt{E} \oplus 0.043$ ( $E$ in $\mathrm{GeV}$ ).

Outside the magnet coil a layer of scintillators is installed, mainly for trigger purposes. The hadron calorimeter (HCAL) made from iron interleaved with limited streamer tubes, serves as return yoke and muon filter, as well. Muon identification is supported by additional muon chambers. The resolution of the HCAL is $\sigma(E) / E=1.12 / \sqrt{E} \oplus 0.21$ ( $E$ in GeV).

\section{L3 Detector}

The subdetectors in the detector of the L3 collaboration (Fig.3.5) [89] are mounted inside a support tube with a diameter of $4.45 \mathrm{~m}$ with the exception of the muon detection system. The muon chambers are only surrounded by a very large low field air magnet $(0.5 \mathrm{~T})$. The 


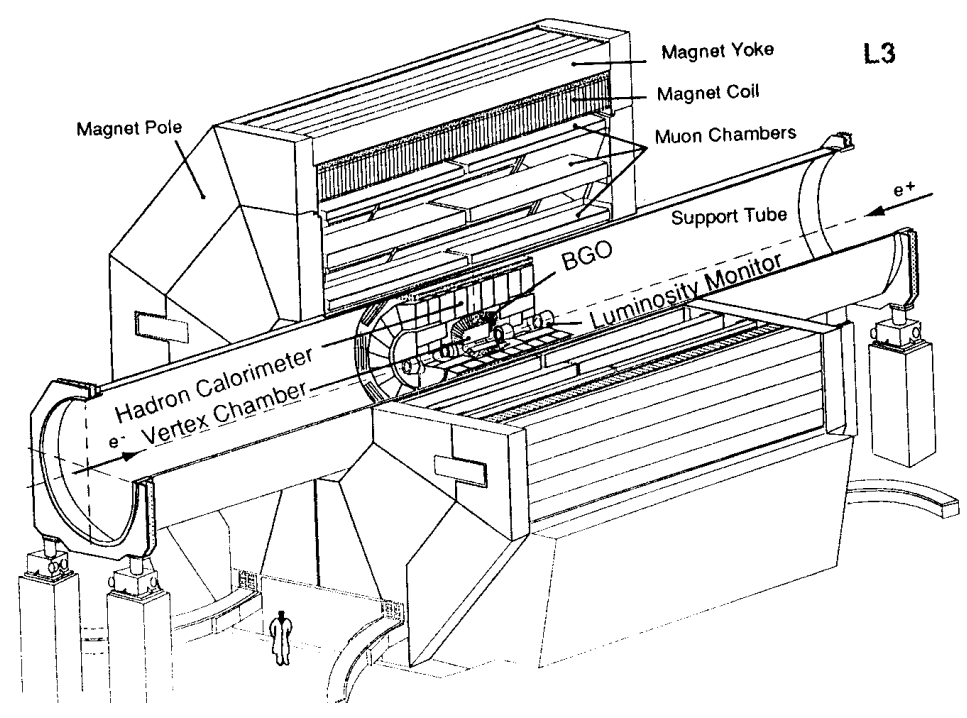

Figure 3.5: L3 detector [89].

coil has an inner diameter of $11.9 \mathrm{~m}$. The size of the magnet allows a long lever arm for the muon momentum measurement. This requires a high precision alignment and monitoring of these chambers.

The tracking system consists of a silicon vertex detector and a central track detector. The latter is a Time Expansion Chamber (TEC) providing 37 points on standard wires for the $r-\phi$ measurement; in addition 14 wires resolving left-right ambiguities. The $z$ coordinate is measured on 11 wires by charge division. The surrounding two cylindrical proportional chambers are designed to provide a good $z$-measurement. With a total lever arm of $0.32 \mathrm{~m}$ the momentum resolution is $\sigma\left(p_{t}\right) / p_{t}^{2}=0.0206 \pm 0.0006\left(p_{t}\right.$ in $\left.\mathrm{GeV} / c\right)$.

Muons in $\mathrm{e}^{+} \mathrm{e}^{-} \rightarrow \mu^{+} \mu^{-}$are measured with the high precision of $\sigma(p) / p \approx 2.5 \%$, with the long lever arm to the muon chambers. Apart from the muon detection, special emphasis was put on a high precision measurement for electromagnetic showers. They are measured in a crystal calorimeter read out by photomultipliers. The crystals of bismuth germanium oxide (BGO) have a shape of a truncated pyramid, $24 \mathrm{~cm}$ long and of $2 \times 2 \mathrm{~cm}^{2}$ at the inner and $3 \times 3 \mathrm{~cm}^{2}$ at the outer end. The energy resolution varies from $5 \%$ at $100 \mathrm{MeV}$ to $1.4 \%$ at high energy.

A layer of scintillation counters is used for time-of-flight measurement. Besides its trigger task, it efficiently rejects cosmic shower events. A uranium calorimeter with proportional wire chambers measures hadronic showers and absorbs most particles except muons. Around this calorimeter a muon filter is mounted, made of brass plates interleaved with five layers of proportional tubes. 


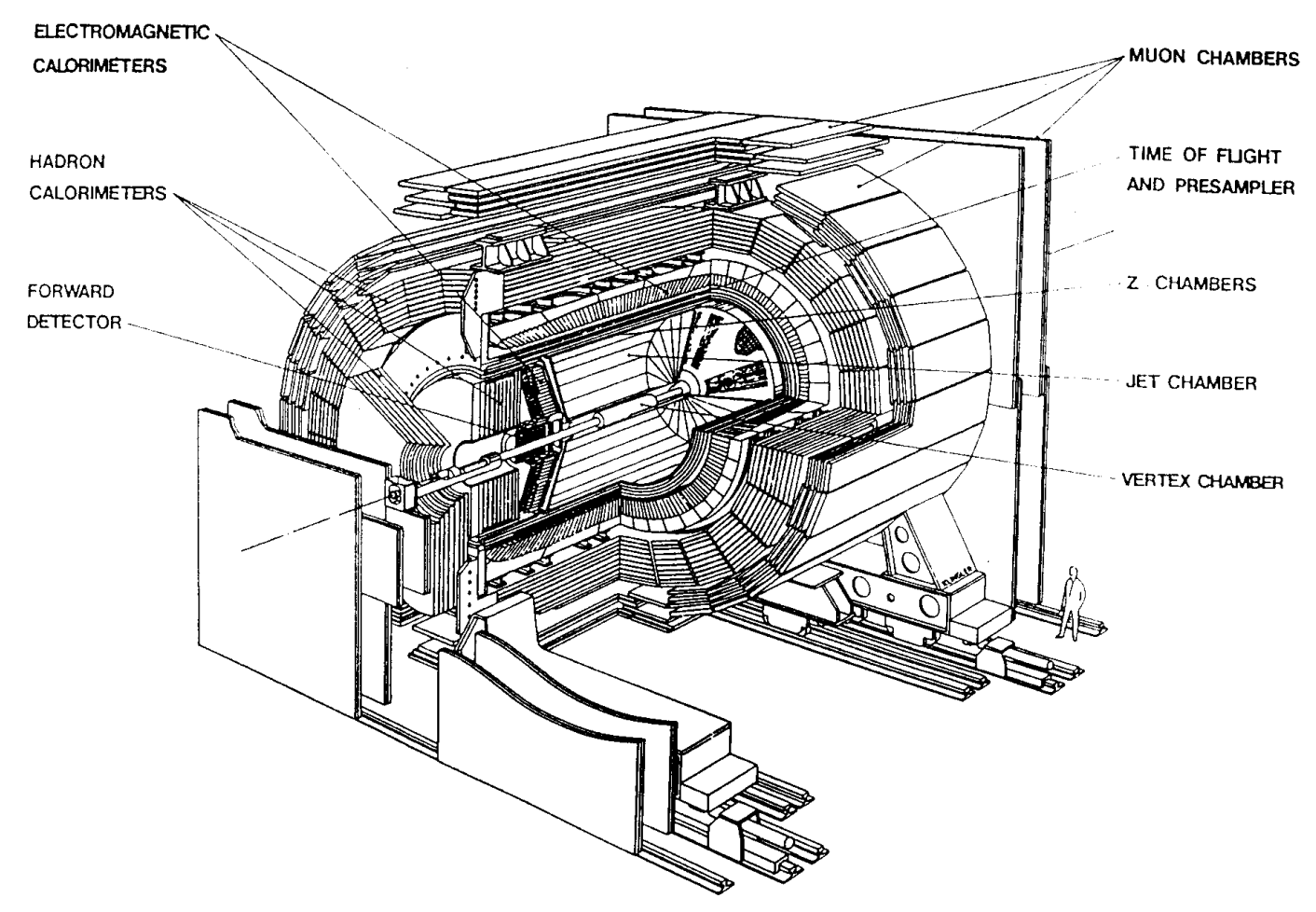

Figure 3.6: OPAL detector [90].

\section{OPAL Detector}

The OPAL detector (Fig.3.6) [90] comprises a tracking system inside a solenoidal magnet of $0.435 \mathrm{~T}$, which consists of a vertex detector a jet-chamber and a $z$-chamber. The new vertex detector of OPAL with two concentric layers of silicon wafers is placed at radii of $6.1 \mathrm{~cm}$ and $7.5 \mathrm{~cm}$. The single hit resolution in $r-\phi$ is $5 \mu \mathrm{m}$, in $z 13 \mu \mathrm{m}$. The main tracking with the jet-chamber provides up to 159 space points $\left(\sigma_{r \phi}=135 \mu \mathrm{m}, \sigma_{z}=6 \mathrm{~cm}\right)$ per track. It allows good particle identification with the energy ionization $\operatorname{loss} \mathrm{d} E / \mathrm{d} x$. The $z$-direction of tracks is substantially improved with information from the $z$-chambers, which are made of modules of drift chambers with 6 staggered anodes strung in $\phi$-direction. The momentum resolution is measured to $\sigma_{p} / p^{2}=0.0022 \mathrm{GeV}^{-1}$.

A time-of-flight system, consisting of scintillation counters, allows particle identification in the momentum range from $0.6 \mathrm{GeV} / c$ to $2.5 \mathrm{GeV} / c$. It is used for triggering and for cosmic shower rejection.

Electromagnetic showers are measured with an assembly of lead glass blocks, with $10 \times 10 \mathrm{~cm}^{2}$ and $37 \mathrm{~cm}$ in depth, read out with photomultipliers. The energy resolution is about $\sigma(E) / E=0.05 / \sqrt{E}(E$ in $\mathrm{GeV})$, when combined with a presampler mounted in front of the calorimeter. Hadrons are measured with nine chambers, limited streamer tubes, interleaved with eight layers of iron plates, where the hadrons may shower. Muons are detected in addition in four layers of drift chambers, the muon chambers. 


\subsection{Event Shape Variables}

From the events recorded by the experiments the hadronic events have to be extracted. For this purpose the characteristics of the events of interest must be known. The global properties, best represented by event shape variables, also serve for model tuning. Of all events recorded at an $\mathrm{e}^{+} \mathrm{e}^{-}$collider, hadronic events have the highest multiplicity. The charged particles will carry about $2 / 3$ of the total centre-of-mass energy, while the neutral ones carry $1 / 3$. The fraction carried away by undetectable neutrinos is small. The particles are in most cases seen in two opposite jets. If hard gluons are radiated, more jets are observed. In order to reconstruct these jets the particles are clustered as function of a jet resolution parameter (e.g., by the DURHAM algorithm [92] $y_{i j}=$ $2 \min \left(E_{i}^{2}, E_{j}^{2}\right)\left(1-\cos \theta_{i j}\right) / E_{v i s}^{2}$; see Section 7.5$)$ : the particle pair with the smallest $y_{i j}$ are combined to form a new 'particle'. The procedure is iterated until a value $y_{c u t}$ is crossed. The number of remaining 'particles' classifies the event as 2-jet event, 3-jet event etc. Their fraction from all events, i.e., the 2-jet rate, 3-jet rate etc., measured at LEP are shown in Figures 3.7 [93] as function of this resolution parameter $y_{\text {cut }}$. The fraction of multi-jet events is lower than at PEP/PETRA energies, because $\alpha_{\mathrm{s}}$ drops with increasing $E_{c m s}$. Three-jet events first observed at the PETRA ring proved the existence of the gluon. Furthermore, the particle flow between the three jets is not the same as is shown in Figure 3.8 [94]. This string effect, which ruled out the independent fragmentation, is due to the higher colour charge of the gluon. A nice illustration of the impact of the gluon is given by the particle density opposite to the gluon, i.e., between quark and anti-quark, as compared to the particle density in radiative two-jet events of similar topology opposite to the photon.

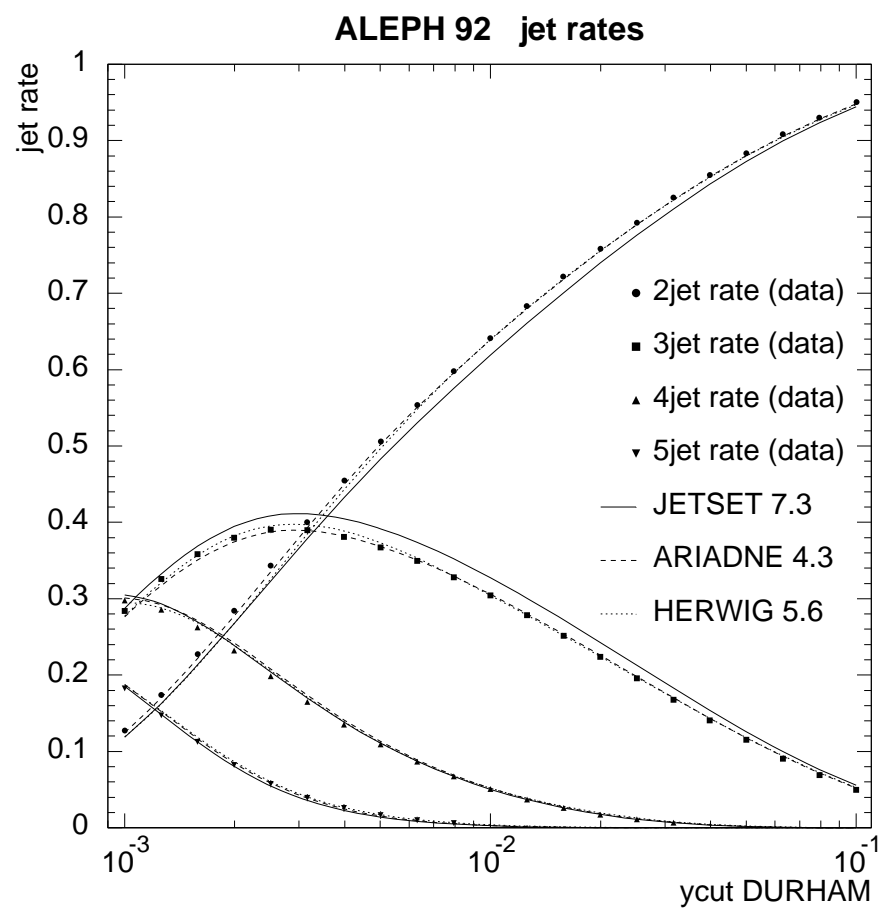

Figure 3.7: Measured n-jet rates in comparison with Monte Carlo Models [93]. 


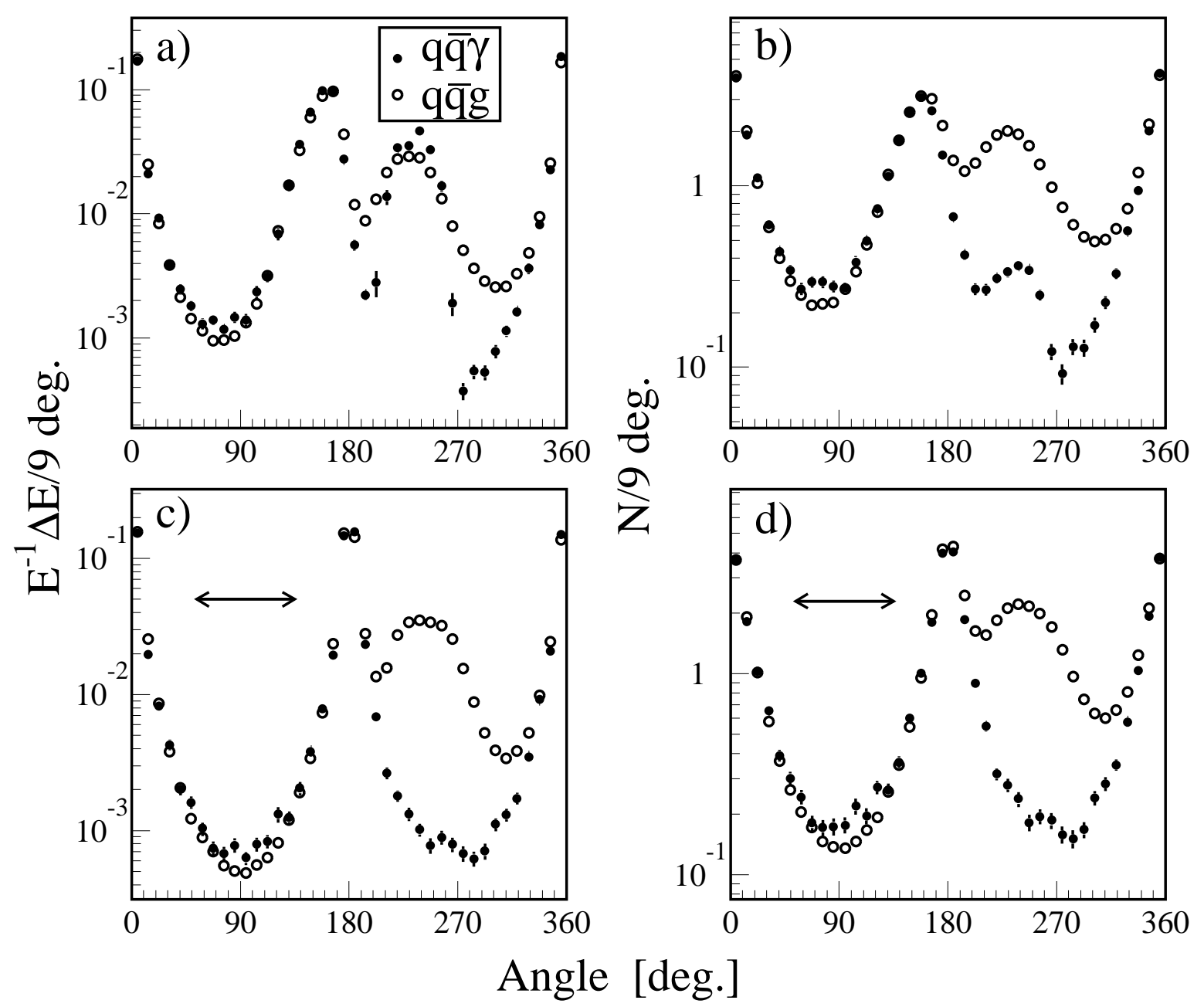

Figure 3.8: (a) Energy and (b) particle flow in three-jet events ( $\mathrm{q} \overline{\mathrm{q}} \gamma$ and $\mathrm{q} \overline{\mathrm{q}} \mathrm{g}$ ) in the laboratory frame; (c) and (d) show the distributions in the q $\bar{q}$ centre-of-mass frame observed by L3, after the photon has been removed [94]. In these distributions, the direction of all particles are projected onto the plane defined by jet 1 an 2, for every event. The angle is measured in this plane starting from jet 1 (jet with the highest energy), increasing through jet 2 to jet 3 (jet with lowest energy or photon) back to jet 1 .

The best known event shape variable is thrust [95]: the direction of the initial quark and anti-quark is fairly well approximated by the thrust-axis, computed from the final state particle momenta. The thrust is defined as $T=\max \left(\Sigma_{i}\left|\mathbf{p}_{\mathbf{i}} \cdot \mathbf{n}\right| / \Sigma_{i}\left|\mathbf{p}_{\mathbf{i}}\right|\right)$. The direction for which $T$ assumes the maximum is the thrust axis. Derived quantities are the $T_{\text {minor }}$ and $T_{\text {major }}$, the latter being the thrust maximized to an axis perpendicular to the thrust axis $\mathbf{n}$; the axis perpendicular to the thrust axis and the major axis is the minor axis. The sphericity $S=3\left(Q_{1}+Q_{2}\right) / 2$ and aplanarity $A=3 / 2 Q_{1}$ are computed from the eigenvalues $Q_{1}<Q_{2}<Q_{3}$ of the normalized $3 \times 3$ sphericity tensor $M_{\alpha \beta}=\Sigma_{i} p_{i \alpha} p_{i \beta} / \Sigma_{i} p_{i}^{2}$, where $\alpha$ and $\beta$ denote the $x, y, z$ momentum component of particle $i$. The unit eigenvector 
$\mathbf{n}_{\mathbf{3}}$ is the sphericity axis (or event axis) and $\mathbf{n}_{\mathbf{2}}$ and $\mathbf{n}_{\mathbf{3}}$ span the event plane [96].

Besides these distributions, the distributions of $p_{t}^{i n}=\left|\mathbf{p} \cdot \mathbf{n}_{\mathbf{2}}\right|$ and $p_{t}^{\text {out }}=\left|\mathbf{p} \cdot \mathbf{n}_{\mathbf{1}}\right|$ for charged particles characterize the shape of hadronic events and are used for tuning the event generators (Refs. [3] to [7]). To characterize the event topology, the $n$-jet rates can be used; however, the differential 2 -jet rate $y_{3}$, the value $y_{i j}$ when the event changes from a 3 -jet event to a 2-jet event, is preferred for tuning. The inclusive momentum distributions and multiplicities of some identified particles are included to further constrain the model parameters, when tuning them.

\subsection{Model Tuning}

The Monte Carlo models described above need input from experiments. The fragmentation process is not fully calculable and has to be simulated with models, which have adjustable parameters. Event shape variables and inclusive distributions and particle rates, corrected for detection efficiency, acceptance, resolution, decays, and initial state photon radiation, are used to tune these phenomenological models. The latter are compared with new data and further improved. Consequently, the model tuning is a steady process of comparison and improvement. As consequence, it should be kept in mind, what is called 'tuned JETSET' or 'tuned HERWIG', is not the same for different years and varies from experiment to experiment [3]-[7].

Furthermore, no model describes all aspects of the data. The result of the adjustment depends on the selection of variables, on the weight given to them, and on whether a spectrum or just the mean value or multiplicity is used; the distributions are not known to the same precision. Particle correlations are not well studied yet, and not used for model tuning; for example Bose-Einstein correlations have an impact on the event shape distributions, though the influence on the scale $\Lambda_{Q C D}$ is small, when the other parameters are retuned $[3,97,98,99]$.

For tuning, one may use a set of parameters which optimizes the main distributions not only with LEP data, but also includes information from $\mathrm{e}^{+} \mathrm{e}^{-}$colliders with lower centre-of-mass energies. However, their impact on the final set is negligible, due to the high accuracy of the LEP data and in this report, the main model parameters are adjusted to LEP data only.

Event shape distributions (see section 3.3) calculated from charged tracks used are: sphericity, aplanarity, thrust, $T_{\text {minor }},-\ln y_{3}$; inclusive distributions $x_{p}=p_{\text {hadron }} / p_{\text {beam }}, p_{t}^{i n}$, $p_{t}^{\text {out }}$ for charged particles; identified particle spectra of $\mathrm{K}_{\mathrm{s}}^{0}, \mathrm{~K}^{ \pm}, \mathrm{p}, \Lambda$; production rates of $\eta, \eta^{\prime}, \rho^{0}, \omega, \phi, \mathrm{K}^{* \pm}, \mathrm{K}^{* 0}, \mathrm{f}_{0}, \mathrm{f}_{2}, \Xi^{-}, \Sigma^{* \pm}, \Xi^{* 0}, \Omega^{-}$. Only data from the ALEPH experiment were used with the exception of $f_{0}$ and $f_{2}$, where DELPHI measurements are taken. For the tuning of the HERWIG model the decuplet baryons are not used [97].

Examples for distributions, that were fitted are shown in Figures 3.9 and 3.10 for event shapes. Comparison with inclusive spectra can be found in Chapter 6 with a general discussion of all particle spectra; a part of these spectra are used for the tuning (see previous paragraph).

The distributions are chosen to give high sensitivity, when tuning the model parame- 


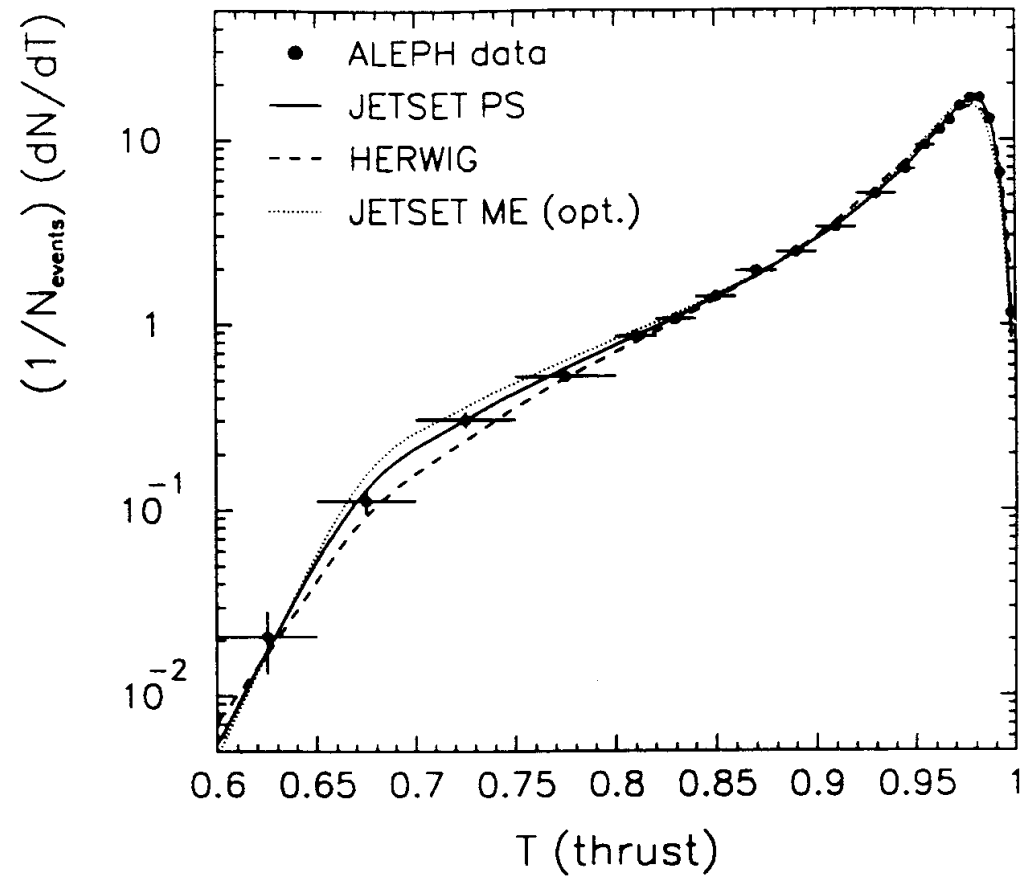

Figure 3.9: Thrust distribution [4].
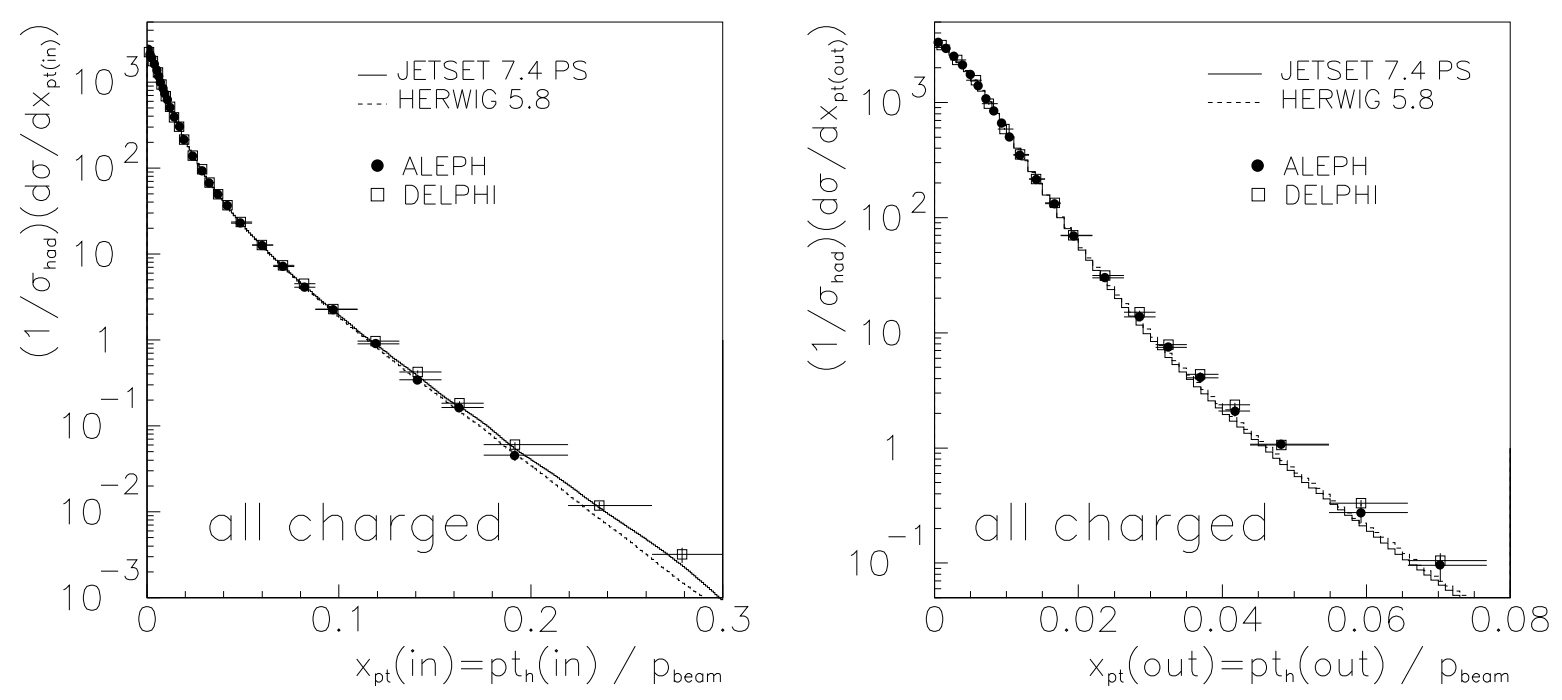

Figure 3.10: $p_{t}^{\text {in }}$ and $p_{t}^{\text {out }}$ distributions. 
ters, while keeping correlations small [3, 5, 99]. The main parameter, the scale $\Lambda_{Q C D}$, a measure for the strength of the strong interaction and related to $\alpha_{\mathrm{s}}$, is constrained by the $n$-jet rate, thrust, and $T_{\text {minor }}$. While thrust is sensitive to the fragmentation in the two-jet region, $T_{\text {minor }}$ is sensitive to the fragmentation, but out of the event plane. $p_{t}^{\text {in }}$ and $p_{t}^{\text {out }}$ are sensitive to the gluon radiation, where the poor agreement of $p_{t}^{\text {out }}$ tells that higher order contributions are missing, and a matching of the models to second order is needed. The most important fragmentation parameters in JETSET are $\sigma_{t}, a, b, \epsilon_{c}$, and $\epsilon_{b}$ as explained in section 2.3. The shower cut-off $Q_{0}$ (denoted $M_{\min }$ in JETSET), below which a parton is not allowed to radiate, influences the inclusive distributions especially for high momenta. A similar sensitivity has the gluon mass $M_{\text {gluon }}$ in HERWIG; this mass is given to the gluon at the end of the shower, when it splits into quarks. Somewhat smaller is the effect of the maximum cluster mass $M_{\text {clust, } \max }$. Two other parameter are important when adjusting the parameters in HERWIG; in a cluster decay the daughters remember the direction of the perturbative quark. The angular distribution is smeared with an exponential in $1-\cos \theta$ with mean $s(\theta)$; the production of strange quarks is suppressed by a factor $\mathcal{P}$ (s-quark). In JETSET many more parameters, which are fixed with inclusive distributions, are implemented (Table 3.3): suppression for mesons to have spin 1 for the various flavours, and mesons with total spin $S$ of the quarks to have orbital angular momentum $L$ between the quarks to form a total spin $J$ of the hadron; $\eta^{\prime}$ suppression (the possibility for $\eta$ suppression is not used by ALEPH, but by other collaborations), s-quark suppression as compared to $\mathrm{u}^{-}$and $\mathrm{d}$ - quark, diquark suppression, a strange diquark suppression (the extra suppression of diquarks with spin 1 with respect to diquarks with spin 0 is taken at its default value: $\left.1 / 3\left(\mathcal{P}\left(u_{1}\right) / \mathcal{P}\left(u_{0}\right)\right)=0.05\right)$, and an extra suppression for diquarks closest to the end of the string. Further switches are set: for the three light quarks, the string fragmentation functions are used, for the heavy flavours, the Peterson ones (Figure 2.5). For baryon production leading baryon suppression is allowed; the popcorn mechanism (Figure 2.7), which allows for the production of a meson M between two baryons $\mathrm{B}$, is used with its default value of $50 \%$ popcorn $(\mathrm{BMB} /(\mathrm{BMB}+\mathrm{BB})=0.5)$. The branching in the parton shower evolution is uniformly distributed in azimuth. For more detailed information, see the original literature [1,2] and [65].

Relevant parameters with their values, which were adjusted and fitted, are listed in Tables 3.3 and 3.4 [97]. These are the values taken in the present analysis for comparison of the inclusive spectra.

\subsection{Event Selection}

We have pointed out in the chapter describing the LEP detectors that all four LEP experiments accept hadronic events from $\mathrm{Z}$ decay with nearly $100 \%$ efficiency. The event selection provides an efficient background rejection, while keeping as many signal events as possible.

The main backgrounds, not rejected by the trigger, are the leptonic decays of the $\mathrm{Z}$ and hadronic events from $\gamma \gamma$ reaction. The effective centre-of-mass energy of the latter is lower then the total available energy: for that reason, $\gamma \gamma$ events are characterized by 


\begin{tabular}{|c|c|c|c|}
\hline parameter & $\begin{array}{l}\text { name in } \\
\text { program }\end{array}$ & $\begin{array}{c}\text { default } \\
\text { value }\end{array}$ & $\begin{array}{l}\text { used } \\
\text { value }\end{array}$ \\
\hline$\Lambda_{Q C D}$ & PARJ(81) & 0.29 & 0.305 \\
\hline$M_{\min }$ & PARJ(82) & 1.0 & 1.43 \\
\hline$\sigma_{t}$ & PARJ(21) & 0.36 & 0.367 \\
\hline $\mathrm{a}$ & PARJ(41) & 0.30 & $0.40^{\dagger}$ \\
\hline $\mathrm{b}$ & PARJ(42) & 0.58 & 0.876 \\
\hline$\epsilon_{c}$ & -PARJ(54) & 0.050 & $0.040^{\dagger}$ \\
\hline$\epsilon_{b}$ & -PARJ(55) & 0.005 & $0.0035^{\dagger}$ \\
\hline $\mathcal{P}(S=1)_{d, u}$ & PARJ(11) & 0.50 & 0.51 \\
\hline $\mathcal{P}(S=1)_{s}$ & PARJ(12) & 0.60 & 0.51 \\
\hline $\mathcal{P}(S=1)_{c, b}$ & PARJ(13) & 0.75 & $0.65^{\dagger}$ \\
\hline $\mathcal{P}\left(J^{P}=2^{+} ; L=1, S=1\right)$ & PARJ(17) & 0. & $0.20^{\dagger}$ \\
\hline $\mathcal{P}\left(J^{P}=1^{+} ; L=1, S=0\right)$ & PARJ(14) & 0 . & $\operatorname{PARJ}(17)^{*} 3 / 5^{\dagger}$ \\
\hline $\mathcal{P}\left(J^{P}=0^{+} ; L=1, S=1\right)$ & PARJ(15) & 0. & $\operatorname{PARJ}(17)^{*} 1 / 5^{\dagger}$ \\
\hline $\mathcal{P}\left(J^{P}=1^{+} ; L=1, S=1\right)$ & PARJ(16) & 0. & $\operatorname{PARJ}(17) * 3 / 5^{\dagger}$ \\
\hline extra $\eta^{\prime}$ suppression & PARJ(26) & 0.40 & $0.25^{\dagger}$ \\
\hline $\mathcal{P}(\mathrm{s}) / \mathcal{P}(\mathrm{u})$ & PARJ( 2) & 0.30 & 0.288 \\
\hline $\mathcal{P}(q q) / \mathcal{P}(q)$ & PARJ( 1) & 0.10 & 0.108 \\
\hline$(\mathcal{P}(\mathrm{su}) / \mathcal{P}(\mathrm{du})) /(\mathcal{P}(\mathrm{s}) / \mathcal{P}(\mathrm{u}))$ & PARJ( 3$)$ & 0.40 & 0.68 \\
\hline leading baryon suppr. & PARJ(19) & 1.0 & 0.53 \\
\hline switch & & & setting \\
\hline fragmentation function & MSTJ(11) & 4 & 3 \\
\hline baryon model & MSTJ(12) & 2 & 3 \\
\hline azimuthal distrib. in ps & $\operatorname{MSTJ}(46)$ & 3 & 0 \\
\hline
\end{tabular}

Table 3.3: Parameters for JETSET 7.4, azimuthal isotropy in parton shower [97]; ${ }^{\dagger}$ adjusted (not fitted).

\begin{tabular}{|c|c|c|c|}
\hline parameter & $\begin{array}{c}\text { name in } \\
\text { program }\end{array}$ & $\begin{array}{c}\text { default } \\
\text { value }\end{array}$ & $\begin{array}{c}\text { used } \\
\text { value }\end{array}$ \\
\hline$\Lambda_{Q C D}$ & QCDLAM & 0.18 & 0.150 \\
$M_{\text {gluon }}$ & RMASS $(13)$ & 0.75 & 0.650 \\
$M_{\text {clust,max }}$ & CLMAX & 3.35 & 3.60 \\
$s(\theta)$ & CLSMR & 0.0 & 0.62 \\
$\mathcal{P}($ s-quark $)$ & PWT $(3)$ & 1.0 & 0.83 \\
\hline
\end{tabular}

Table 3.4: Parameters for HeRwig 5.8 [97]. 
a lower energy and a lower charged multiplicity observed in the detector. The leptonic decays have just two charged tracks for $\mathrm{e}^{+} \mathrm{e}^{-}$and $\mu^{+} \mu^{-} ; \tau^{+} \tau^{-}$decays may have a few charged track (85.5\% of the $\tau$ decays are one-prong decays, $14.4 \%$ are three-prong decays). The energy seen in the detector is lower, because the neutrinos escape detection (Figure $3.11[100])$.

Requirements on the visible energy (e.g., > $15 \mathrm{GeV}$ ) and number of tracks originating from the primary collision point (at least five tracks) are applied. Additional selection cuts may be included such as cuts on the polar angle of the tracks $\left(>20^{\circ}\right)$ and the sphericity axis $\left(>35^{\circ}\right)$ with respect to the beam line. These cuts ensure that the event is safely contained in the detector acceptance.

$80 \%$ of hadronic events are typically selected with a negligible background from tau decays and with an admixture of two-photon events of less than $0.3 \%$ [4].

The track selection reduces the signal, which is then corrected for the cross section extraction. Monte Carlo models are used for possible particle misidentification, background, resolution effects and bias in the event selection; extrapolation to the unobserved region in momentum is performed. Corrections for initial state radiation at the $\mathrm{Z}$ pole, important for the total cross sections, can safely be neglected for the inclusive particle production rates and momentum spectra.

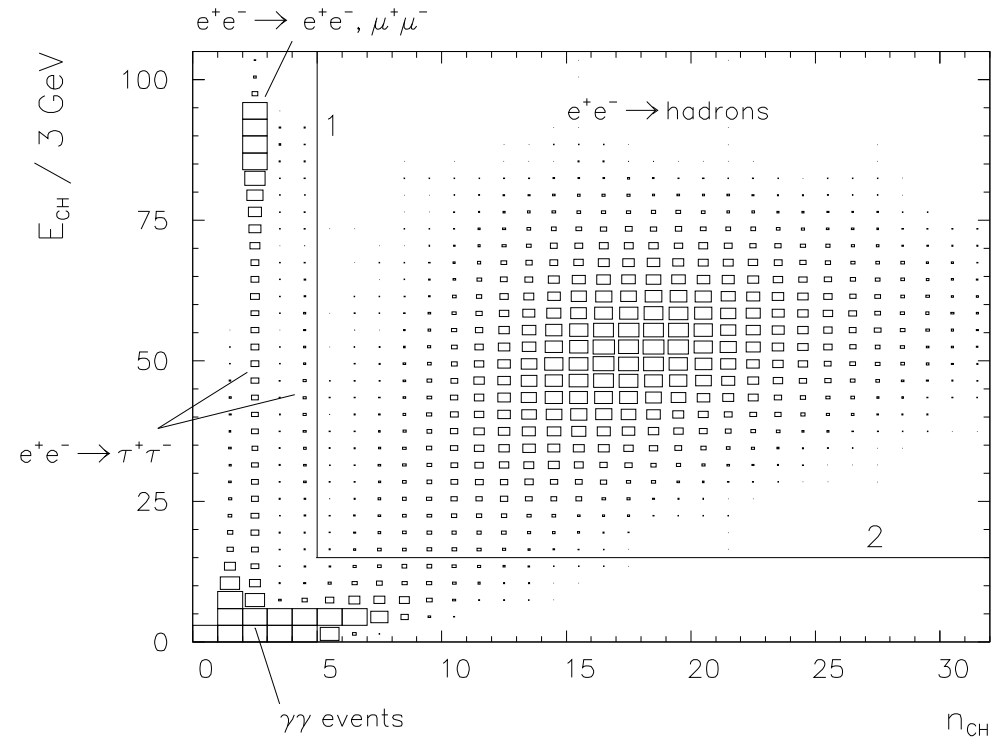

Figure 3.11: Background rejection: shown is the energy of the charged particles with respect to the number of charged particles. Line 1 and 2 indicate the selection cuts used for the selection of hadronic events, rejecting di-lepton and $\gamma \gamma$ events [100]. 


\section{Chapter 4}

\section{Particle Identification}

\subsection{Detection Methods, Particle Identification}

Stable charged particles or charged particles with lifetimes longer than $10 \mathrm{~ns}$, i.e. particles $\left(\pi^{ \pm}, \mathrm{K}^{ \pm},(\mathrm{p}, \overline{\mathrm{p}}), \mathrm{e}^{ \pm}, \mu^{ \pm}\right)$with decay lengths of the order or larger than the dimension of the tracking chamber, are identified by measuring the ionization loss $\mathrm{d} E / \mathrm{d} x$. All four LEP experiments use this means of particle identification. The $\mathrm{d} E / \mathrm{d} x$ measurements are normalized using minimum ionizing pions, which also allows the determination of $\sigma_{\mathrm{d} E / \mathrm{d} x}$ the expected $\mathrm{d} E / \mathrm{d} x$ resolution. In the DELPHI experiment the ring imaging Cherenkov detector (RICH) provides a good separation of particle species, especially for high momenta (Figure 4.1).

Stable neutral particles $\left(n, K_{1}^{0}\right)$ are difficult to isolate and measure in a multihadronic environment. They are seen as clusters in the hadron calorimeter. This has a worse resolution than the electromagnetic calorimeter and the tracking detectors. The clusters have been used for global analyses, e.g., $\alpha_{\mathrm{s}}$ determinations with energy flow objects. Inclusive spectra, however, have never been measured. The DELPHI collaboration has succeeded using neutrons indirectly as a confirmation in $\Sigma^{+} \rightarrow \mathrm{n} \pi^{+}$decays.

On the other hand, neutral particles decaying to photons (such as $\pi^{0}$ or $\eta$ ) are well measured with the electromagnetic calorimeter, if the granularity is fine enough for good separation of the showers.

Neutral particles with light flavours ( $\mathrm{V}^{0}$, e.g., $\Lambda, \mathrm{K}_{\mathrm{s}}^{0}$ ), have average decay lengths ranging from $\mathrm{cm}$ to metres in the laboratory frame. They are detected by a secondary vertex in the tracking chamber. Two tracks being consistent as coming from a common point different from the primary $\mathrm{e}^{+} \mathrm{e}^{-}$collision point are selected with little background, as can be seen in an invariant mass distribution of such pairs. A kinematic fit, which uses the fact that most of these light flavoured hadrons originate from the primary vertex, may improve the signal to background ratio.

Charm and bottom flavoured hadrons, and some of the strange baryons, decay into several charged particles with decay lengths shorter than $\mathrm{V}^{0}$ 's. Nevertheless, often a secondary vertex is reconstructed. This is achieved by the high precision of special vertex detectors. With resolutions of several $\mu \mathrm{m}$, vertices close to the beam line (and even inside the beam pipe) are measured with an accuracy of several $100 \mu \mathrm{m}$. Charged strange 

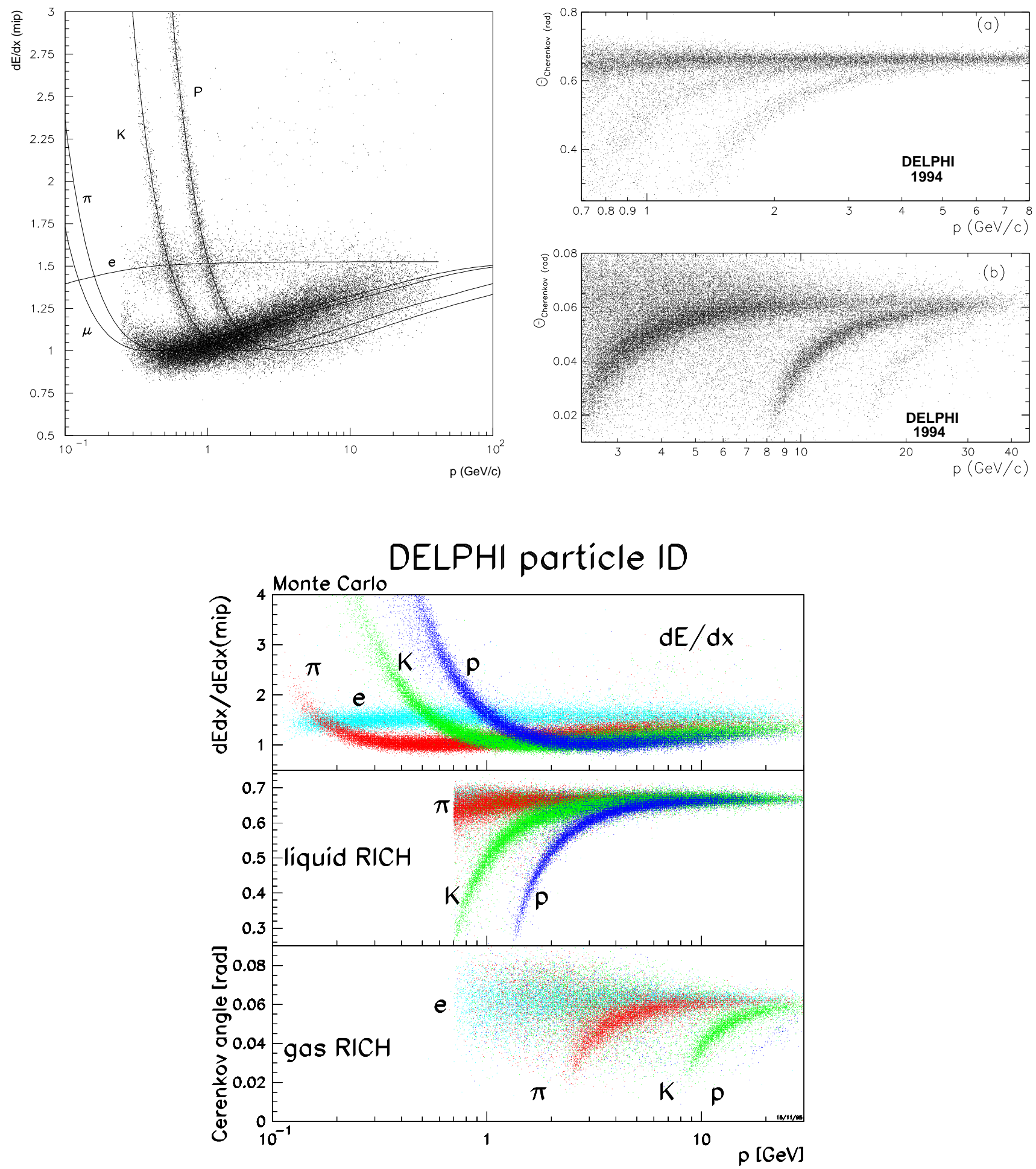

Figure 4.1: Particle identification with the DELPHI detector. Shown are the specific ionizations normalized to minimum ionizing pions and the Cherenkov angles in the RICHes. The lower diagrams show the corresponding distributions for simulated events [88]. 
baryons $\left(\Sigma^{ \pm}\right)$have also been observed by detecting a kink of a track in the tracking chamber; the location of the kink represents the secondary vertex, then.

Similarly $\mathrm{e}^{+} \mathrm{e}^{-}$pairs are seen due to photons which have converted in the detector material in front of or within the tracking chamber. Only a few photons $(5-10 \%)$ convert with the majority seen as showers in the electromagnetic calorimeter. Combining pairs of photons, neutral pions, the most copiously produced neutral particles, are reconstructed.

Other short-lived particles are detected as a peak in the invariant mass distribution of their daughters. The charged daughters can be restricted to those from the primary vertex. The combinatorial background under these peaks is high, because the resonances may be wide or the decay products include neutrals (e.g., $\pi^{0}$ ).

\subsection{Signal Extraction}

In order to get a reliable cross section for the production of a particle, several conditions have to be fulfilled in addition to the use of the procedures described in the previous section.

The shapes of the resonances depend on the spin of parent and daughters and the decay products. Functions describing these shapes can be found in Ref. [101]. Masses and widths may be found in Ref. [79]. Possible distortions, e.g., from acceptance or BoseEinstein correlations, must be accounted for. The resolution plays an important role for narrow resonances. The resolution may be further improved by a kinematic fit and by fitting mass differences $\left(m\left(\Sigma^{0}\right)-m(\Lambda)\right.$ for $\left.\Sigma^{0} \rightarrow \Lambda \gamma\right)$, when the daughters themselves are reconstructed from their decay.

The shape and height of the background is usually estimated by fitting a smooth function. The background may be huge in some of the analyses. Therefore, the like-sign invariant charged pion distribution is subtracted from the unlike-sign one in extracting the $\rho^{0}$, because the like-sign distribution is an estimation for combinatorial background in the unlike-sign one. While increasing the statistical error by this procedure, it reduces the systematics and the remaining background is easier to parameterize. Another method is the track or event mixing technique. Care must be taken when reflections are close to the signal as illustrated with the $\mathrm{K}^{* 0}$ analysis in Figure 4.2 . The $\omega$ and $\rho^{0}$ appear as structure in the like-sign subtracted $\pi^{ \pm} \mathrm{K}^{\mp}$ mass spectrum, because pions are misidentified as kaons. The smoothness and amount of background below the resonance in narrow resonances is often checked using distributions of the side bands or wrong-sign combinations in weak decays.

The extraction of the production cross section is done in intervals of momentum or energy of the particles produced, and the inclusive distribution is obtained. The procedures are backed up by studies of Monte Carlo events, which contain the physics processes to the best present knowledge, and which are needed to compute the acceptance corrections. Corrections have to be applied for particles, regarded as unstable (e.g., $\Lambda$ ), that leave the tracking devices before their decay; or (almost stable) particles (e.g., $\pi^{ \pm}$), which decay. Further corrections are needed for; nuclear interactions within the detector material; undetected low momentum tracks $(p<150 \mathrm{MeV} / c)$; track losses from the reconstruction or 


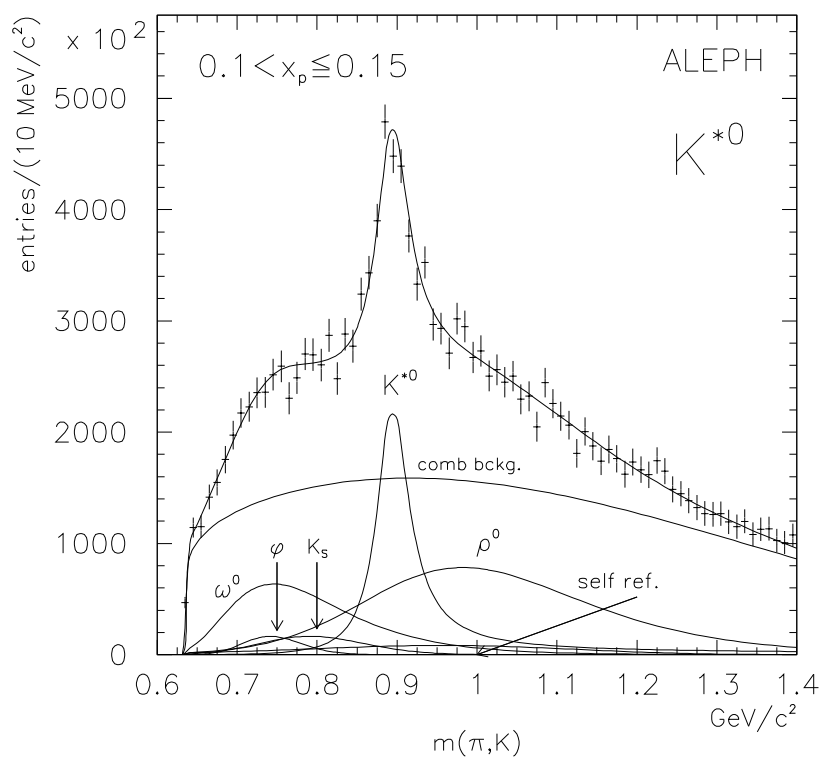

Figure 4.2: Invariant mass spectrum $m\left(\mathrm{~K}^{ \pm} \pi^{\mp}\right)$ (like-sign subtracted). The data (crosses) are well described by the fit (solid curve). The contributions from signal, reflections, and combinatorial background are shown as well [23].

fake tracks from track spiraling or splitting. 


\section{Chapter 5}

\section{Average Hadron Multiplicities}

\subsection{Experimental Data}

In Table 5.1 the measurements on average hadron multiplicities per hadronic $\mathrm{Z}$ decay at $\sqrt{s}=91 \mathrm{GeV}$ are listed. All four LEP experiments have studied the inclusive charged particle momentum distribution (The decay products of particles with lifetime shorter than $10^{-9} \mathrm{~s}$, such as $\mathrm{K}_{\mathrm{s}}^{0},(\Lambda, \bar{\Lambda})$ are included.). The high particle multiplicity is the primary characteristic of hadronic events. The number of charged stable particles is easily accessible and is determined with high accuracy. It is therefore an important quantity all Monte Carlo models have be tuned to. The energy dependence of the charged particle multiplicity is predicted by the MLLA. A comparison with multiplicities measured at lower energy provides a crucial test. The compilation performed [11] showed good agreement.

The comparison between the LEP experiments of the total rates, i.e., the average multiplicities of particles per event, shows good agreement. For stable particles as well as for copiously produced unstable particles, such as pseudoscalar mesons and vector mesons, the various measurements agree. The rate of the $\eta^{\prime}$ is an exception in this respect. Up to now, only four mesons $\left(f_{0}, f_{2}, f_{2}^{\prime}\right.$, and $\left.K_{2}^{* 0}\right)$ with orbital angular momentum $\mathrm{L}=1$ are measured; the errors are $20 \%$ to $30 \%$ with the total rates obtained extrapolating measurements in a limited momentum interval. It has to be pointed out, however, that the rates are higher than one had expected. Some experiments had neglected $\mathrm{L}=1$ meson production until recently, when tuning their models.

The situation is less clear for the baryons. The proton with the highest cross section is only in approximate agreement between the experiments. This is surprising, because all LEP experiments have good particle identification measuring the energy loss simultaneously with momentum for charged particles (see Figure 4.1); the band for the proton is separated from the kaon and pion band. The results for strange baryons are compatible, see e.g., the $\Lambda$ baryon, but baryons with low production probability need more investigation. The differences for $\Sigma^{* \pm}$ and the $\Omega^{-}$may be understood, because the extraction is difficult. The $\Sigma^{* \pm}$ is a wide resonance with a high combinatorial background underneath. The $\Omega^{-}$has a small production rate and the reconstructed signal may be close to the kinematical limit (for $\Omega^{-} \rightarrow \Lambda \mathrm{K}^{-}$), when the resolution of the detector in the invariant mass is worse than $\approx 15 \mathrm{MeV} / c^{2}$. The knowledge of the $\Omega^{-}$rate, however, is a corner-stone for 


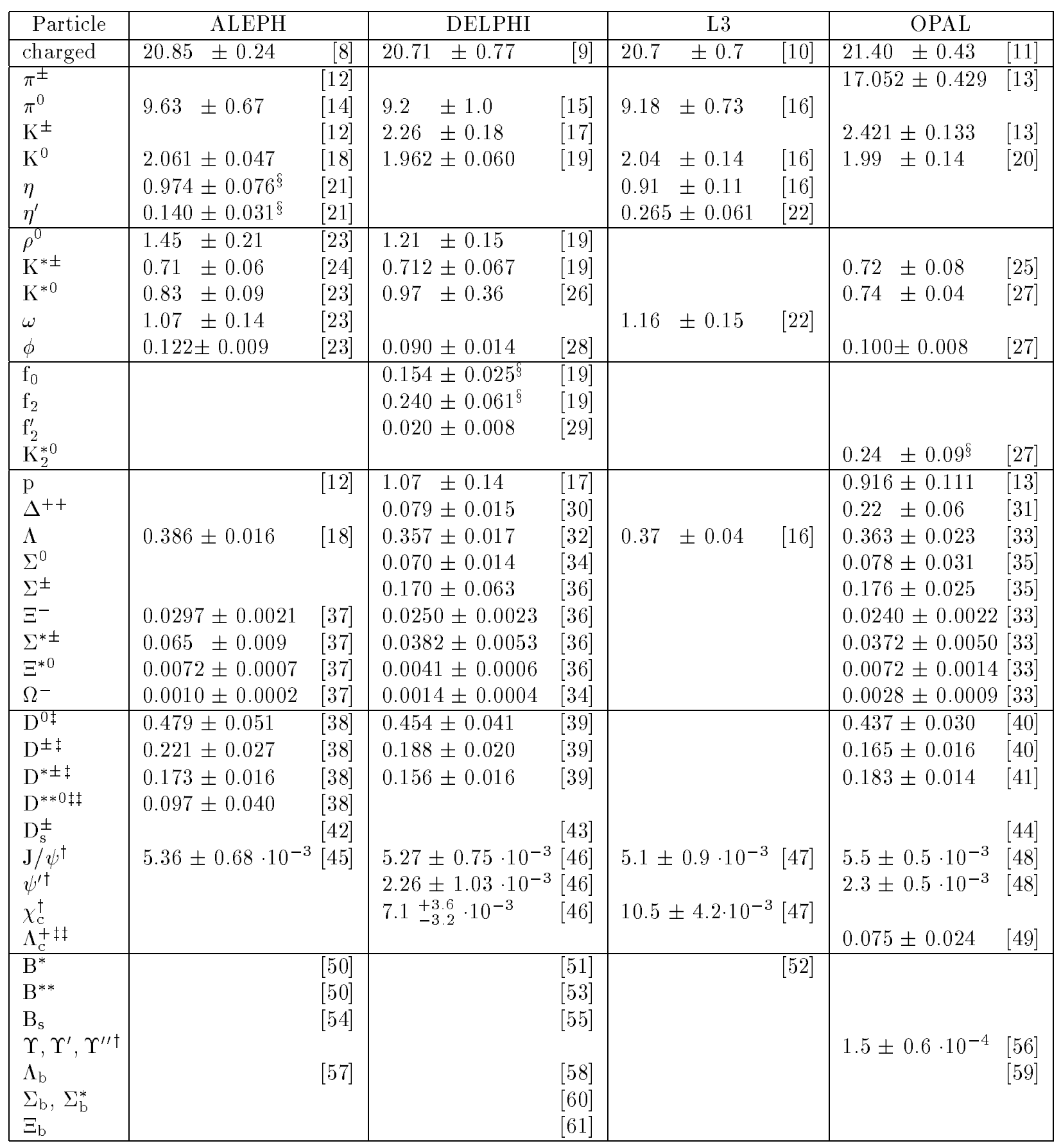

§: extrapolated with JETSET

$\ddagger$ D-mesons: Corrected with new branching ratios [79]. ${ }^{\ddagger \ddagger} \mathrm{D}^{* * 0}, \Lambda_{c}^{+}$: Corrected with branching ratios.

$\dagger$ charmonia, bottomonia: Corrected for hadronic width: numbers in publications are given as branching ratios of the $\mathrm{Z}$.

Table 5.1: Average hadron multiplicities measured by the four LEP experiments. The errors are statistical and systematic errors added in quadrature; more details (statistical errors etc.) can be found in Chapter 6. For most of the heavy particles, production rates are given multiplied by branching ratios, which mostly have large uncertainties; the measurements are given in Chapter 6 . 
the understanding of the strangeness suppression in the fragmentation of baryons. The present measurement of the $\Omega^{-}$at LEP is close to the expected rate from the Monte Carlo simulation, contrary to the high rate measured at the PETRA storage ring [102]. The measurements of the light baryon $\Delta^{++}$with width $\Gamma \approx 120 \mathrm{MeV} / c^{2}$, certainly need clarification.

Some of the charm and bottom particles have been measured or observed at LEP. Of these the D-mesons are the most frequently produced particles, and their fragmentation functions have been measured. While the numbers of events with identified bottom baryons and mesons is small, they have been numerous enough to allow a first direct proof of time dependent mixing in the bottom sector. The production probabilities of B-hadrons published are given for specific decay channels, where the branching ratios are uncertain, or as relative production rates. We refer to the discussion in Section 6.10, where the numbers are listed as published by the experiments.

\subsection{Comparison with Monte Carlo Models}

In Table 5.2 the average particle multiplicities measured by the four LEP experiments are compared with the Monte Carlo model prediction. The values for LEP are given with their total errors. When averaging the LEP results, the final error is multiplied by $\sqrt{\chi^{2}}$, if $\chi^{2}$ is larger than 1. The predictions of the JETSET and HERWIG models are those obtained with the tuned parameter settings as given in Section 3.4.

The total charged particle multiplicity agrees with the data for both models; the prediction of JETSET is slightly too low. The charged particle momentum distribution is used in the model tuning, therefore the agreement is no surprise for JETSET; it is, however, for HERWIG which has fewer parameters.

The meson octets, pseudoscalar and vector meson octet, are well described; they had been studied already at lower centre-of-mass energies, because of their high production probability, and are well understood. The $\eta$ and $\eta^{\prime}$ raised some concern, while tuning the models, because the predictions were too high with the standard parameter settings. The rates could be influenced by changing their mixing angle used in the Monte Carlo models; the effect is too low, however. The choice to introduce new parameters for $\eta$ and $\eta^{\prime}$ suppression improved the agreement between the data and the models; it is, however, not a satisfactory solution for the understanding of particle production.

For the mesons with orbital angular momentum little information is available, and therefore not much input to the models is provided. Only four species have been measured, each one only by one experiment, with large errors. Nevertheless, the agreement is good for $f_{2}$ and $f_{2}^{\prime}$; the $K_{2}^{* 0}$ rate is too small, as is the $f_{0}$ rate in JETSET, which is not generated at all in HERwig.

For baryons the situation is similar. The octet baryons, which are most frequently produced, are nicely reproduced by both models. An exception is the $\Xi^{-}$, where the rate in the HERwIG generator is twice as large as in the data. For the decuplet baryons the situation is worse. JETSET is closer to the data; HERWIG, however, overestimates the strange baryons. For the $\Delta^{++}$, the two LEP measurements differ by a factor three, one 


\begin{tabular}{|l|l|l|l|l|l|}
\hline Particle & \multicolumn{1}{|c|}{ LEP } & JETSET 7.4 & HERWIG 5.8 & Experiment & Figure \\
\hline charged & $20.94 \pm 0.19$ & 20.59 & 20.67 & ADLO & $6.3,6.4$ \\
\hline$\pi^{ \pm}$ & $17.05 \pm 0.43$ & 16.86 & 17.26 & O & $6.3,6.4$ \\
$\pi^{0}$ & $9.38 \pm 0.45$ & 9.65 & 9.61 & ADL & 6.9 \\
$\mathrm{~K}^{ \pm}$ & $2.36 \pm 0.11$ & 2.14 & 2.08 & DO & $6.3,6.4$ \\
$\mathrm{~K}^{0}$ & $2.009 \pm 0.027$ & 2.083 & 2.044 & ADLO & 6.7 \\
$\eta^{\dagger}$ & $0.953 \pm 0.076$ & 1.062 & 0.990 & AL & 6.9 \\
$\eta^{\prime \dagger}$ & $0.166 \pm 0.051$ & 0.155 & 0.132 & AL & 6.9 \\
\hline$\rho^{0}$ & $1.29 \pm 0.12$ & 1.286 & 1.330 & AD & 6.10 \\
$\mathrm{~K}^{* \pm}$ & $0.713 \pm 0.039$ & 0.774 & 0.692 & ADO & 6.10 \\
$\mathrm{~K}^{* 0}$ & $0.757 \pm 0.036$ & 0.777 & 0.693 & ADO & 6.10 \\
$\omega$ & $1.11 \pm 0.10$ & 1.261 & 0.861 & AL & 6.10 \\
$\phi$ & $0.103 \pm 0.008$ & 0.105 & 0.092 & ADO & 6.10 \\
\hline $\mathrm{f}_{0}^{\dagger}$ & $0.154 \pm 0.025$ & 0.046 & 0. & D & 6.11 \\
$\mathrm{f}_{2}^{\dagger}$ & $0.240 \pm 0.061$ & 0.209 & 0.226 & D & 6.11 \\
$\mathrm{f}_{2}^{\prime}$ & $0.020 \pm 0.008$ & 0.021 & 0.024 & D & 6.11 \\
$\mathrm{~K}_{2}^{* 0 \dagger}$ & $0.24 \pm 0.09$ & 0.127 & 0.131 & O & - \\
\hline $\mathrm{p}$ & $0.975 \pm 0.087$ & 1.068 & 1.047 & DO & $6.3,6.4$ \\
$\Delta^{++}$ & $0.087 \pm 0.033$ & 0.159 & 0.234 & DO & 6.12 \\
$\Lambda$ & $0.370 \pm 0.010$ & 0.379 & 0.461 & ADLO & 6.7 \\
$\Sigma^{0}$ & $0.071 \pm 0.013$ & 0.087 & 0.063 & DO & 6.13 \\
$\Sigma^{ \pm}$ & $0.175 \pm 0.029$ & 0.167 & 0.145 & DO & 6.13 \\
$\Xi^{-}$ & $0.0264 \pm 0.0018$ & 0.033 & 0.061 & ADO & 6.13 \\
$\Sigma^{* \pm}$ & $0.0415 \pm 0.0 .0067$ & 0.068 & 0.159 & ADO & 6.13 \\
$\Xi^{* 0}$ & $0.0056 \pm 0.0011$ & 0.0065 & 0.0322 & ADO & 6.13 \\
$\Omega^{-}$ & $0.0011 \pm 0.0003$ & 0.0012 & 0.0096 & ADO & 6.13 \\
\hline $\mathrm{D}^{0}$ & $0.450 \pm 0.022$ & 0.500 & 0.556 & ADO & - \\
$\mathrm{D}^{ \pm}$ & $0.182 \pm 0.015$ & 0.219 & 0.252 & ADO & - \\
$\mathrm{D}^{* \pm}$ & $0.172 \pm 0.009$ & 0.216 & 0.228 & ADO & 6.14 \\
$\mathrm{D}^{* * 0}$ & $0.097 \pm 0.040$ & 0.038 & 0.020 & A & - \\
$\mathrm{J}^{\dagger} \psi$ & $0.0054 \pm 0.0003$ & 0.0042 & 0. & ADLO & 6.17 \\
$\psi^{\prime}$ & $0.0023 \pm 0.0004$ & 0. & 0. & DO & - \\
$\chi_{\mathrm{c}}$ & $0.0084 \pm 0.0026$ & 0.0023 & 0. & DL & - \\
$\Upsilon, \Upsilon^{\prime}, \Upsilon^{\prime \prime}$ & $0.00015 \pm 0.00006$ & 0.000002 & 0. & $\mathrm{O}$ & - \\
$\Lambda_{c}^{+}$ & $0.075 \pm 0.024$ & 0.034 & 0.023 & $\mathrm{O}$ & - \\
\hline
\end{tabular}

$\dagger$ range extrapolated with JETSET.

Table 5.2: Average hadron multiplicities measured at LEP in comparison with Monte Carlo models. The number of the figures showing the comparison of the momentum spectra are given in the last column.

measurement would confirm JETSET, while the other is lower, which, however, agrees with the shape of JETSET in the $\Delta^{++}$momentum distribution.

The production of heavy flavour hadrons, which hardly occurs in the soft fragmentation since these particles mostly contain the primary quark, is reproduced by the models. The description is not so much a problem of the shower evolution, but rather a problem of the 
flavour composition at the $\mathrm{Z}$ pole, the probability for heavy flavour production from the corresponding quark, and the branching ratios involved.

\subsection{Measurements at Lower Centre-of-Mass Energy}

Measurements of average particle multiplicities at lower centre-of-mass energy and their spectra can be found in Refs. $[62,79,103,104,105,106]$. In Table 5.3 the LEP averages of light hadrons are compared to measurements at lower energy. Heavy flavour rates, however, should be compared with caution: the flavour composition at LEP is different ( $22 \%$ for down-type flavours and $17 \%$ for up-type flavours) from the flavour composition at lower energies (proportional to the electric quark-charge squared). The ratios for light flavoured hadrons are displayed in Figure 5.1 in comparison with the JETSET and HERWIG model predictions, simulated with $E_{c m s}=10 \mathrm{GeV}, 32 \mathrm{GeV}$, and $91 \mathrm{GeV}$.

\begin{tabular}{|c|c|c|c|}
\hline Particle & $10 \mathrm{GeV}$ & $29-35 \mathrm{GeV}$ & $91 \mathrm{GeV}$ \\
\hline$\pi^{ \pm}$ & $\begin{array}{ll}6.6 & \pm 0.2\end{array}$ & $10.3 \pm 0.4$ & $17.05 \pm 0.19$ \\
\hline$\pi^{0}$ & $3.2 \pm 0.3$ & $5.6 \pm 0.3$ & $9.38 \pm 0.45$ \\
\hline$K^{ \pm}$ & $0.90 \pm 0.04$ & $1.48 \pm 0.09$ & $2.36 \pm 0.11$ \\
\hline$K^{0}$ & $0.91 \pm 0.05$ & $1.48 \pm 0.07$ & $2.009 \pm 0.027$ \\
\hline$\eta$ & $0.20 \pm 0.04$ & $0.61 \pm 0.07$ & $0.953 \pm 0.096$ \\
\hline$\eta^{\prime}$ & $0.03 \pm 0.01$ & $0.26 \pm 0.10$ & $0.166 \pm 0.051$ \\
\hline$\rho^{0}$ & $0.35 \pm 0.04$ & $0.81 \pm 0.08$ & $1.29 \pm 0.12$ \\
\hline $\mathrm{K}^{* \pm}$ & $0.27 \pm 0.03$ & $0.64 \pm 0.05$ & $0.713 \pm 0.039$ \\
\hline $\mathrm{K}^{* 0}$ & $0.29 \pm 0.03$ & $0.56 \pm 0.06$ & $0.757 \pm 0.036$ \\
\hline$\omega$ & $0.30 \pm 0.08$ & & $1.11 \pm 0.10$ \\
\hline$\phi$ & $0.044 \pm 0.006$ & $0.085 \pm 0.011$ & $0.103 \pm 0.008$ \\
\hline$f_{0}$ & $0.024 \pm 0.006$ & $0.11 \pm 0.04$ & $0.154 \pm 0.025$ \\
\hline $\mathrm{f}_{2}$ & $0.09 \pm 0.02$ & $0.14 \pm 0.04$ & $0.240 \pm 0.061$ \\
\hline $\mathrm{f}_{2}^{\prime}$ & & & $0.020 \pm 0.008$ \\
\hline $\mathrm{K}_{2}^{* 0}$ & & $0.12 \pm 0.06$ & $0.24 \pm 0.09$ \\
\hline $\mathrm{K}_{2}^{* \pm}$ & & $0.09 \pm 0.03$ & \\
\hline $\mathrm{p}$ & $0.253 \pm 0.016$ & $0.640 \pm 0.050$ & $0.975 \pm 0.087$ \\
\hline$\Delta^{++}$ & $0.040 \pm 0.010$ & & $0.087 \pm 0.033$ \\
\hline$\Lambda$ & $0.080 \pm 0.007$ & $0.205 \pm 0.010$ & $0.370 \pm 0.010$ \\
\hline$\Sigma^{0}$ & $0.023 \pm 0.008$ & & $0.071 \pm 0.013$ \\
\hline$\Sigma^{ \pm}$ & & & $0.175 \pm 0.029$ \\
\hline$\Xi^{-}$ & $0.0059 \pm 0.0007$ & $0.0176 \pm 0.0027$ & $0.0264 \pm 0.0018$ \\
\hline$\Sigma^{* \pm}$ & $0.0106 \pm 0.0020$ & $0.033 \pm 0.008$ & $0.0415 \pm 0.0067$ \\
\hline$\Xi^{* 0}$ & $0.0015 \pm 0.0006$ & & $0.0056 \pm 0.0011$ \\
\hline$\Omega^{-}$ & $0.0007 \pm 0.0004$ & $0.014 \pm 0.007$ & $0.0011 \pm 0.0003$ \\
\hline$\Lambda^{*}$ & $0.008 \pm 0.002$ & & \\
\hline
\end{tabular}

Table 5.3: Average light particle multiplicities measured at lower centre-of-mass energy [79] in comparison with LEP results. 

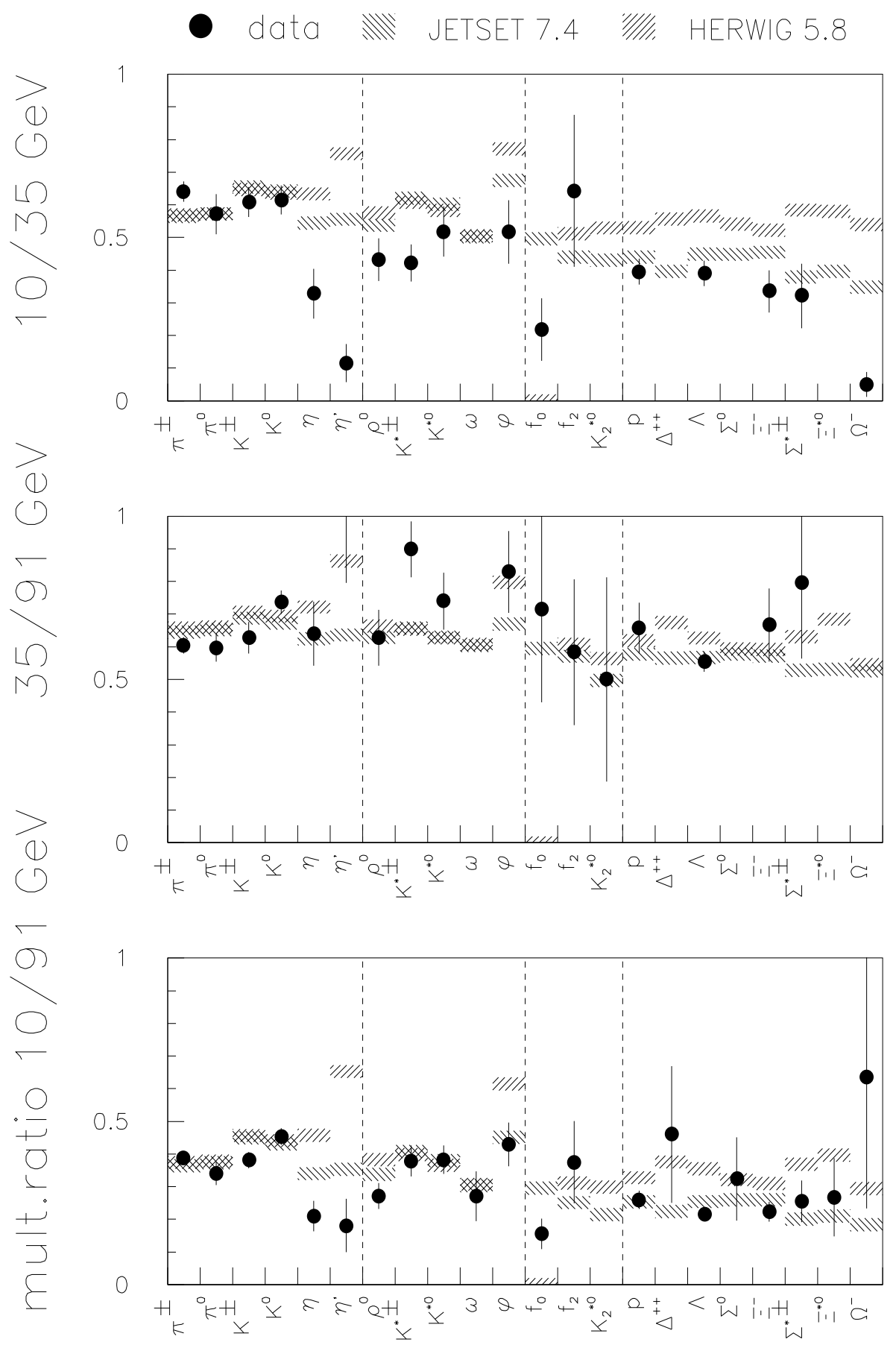

Figure 5.1: Multiplicity ratios of particle production rates at different $E_{c m s}$ for pseudoscalar, vector, and $L=1$ mesons and for baryons in comparison with the JETSET and HERWIG model predictions. The ratios for $\eta^{\prime}$ and $\Omega^{-}$for $35 \mathrm{GeV} / 91 \mathrm{GeV}$ are offscale $(1.57 \pm 0.77$ and $12 \pm 7)$. No measurements exist for $\omega, \Delta^{++}$, and $\Sigma^{0}$ at $35 \mathrm{GeV}$. 
The most obvious aspect is the increase of all particle production rates reflecting the increase of the total hadron multiplicities with the centre-of-mass energy. The multiplicity per hadronic event at LEP are about $50 \%$ higher than at PEP/PETRA. This is expected by MLLA and LPHD and well reproduced by the Monte Carlo simulation; therefore, the tuning of the models excluded the low energy measurements, while they had been used at the LEP startup and in the first years of running.

A few deviations from this behaviour of increased multiplicity are seen. The $\eta^{\prime}$ rate is lower at LEP than that measured at PEP/PETRA energies! A view to the other particles and especially the results at $\sqrt{s}=10 \mathrm{GeV}$ indicates that the $\eta^{\prime}$ rate at PEP/PETRA was measured too high; the measurement of the $\eta^{\prime}$ rate has an error of $40 \%$. A similar observation is made for the $\Omega^{-}$, which has a surprisingly large rate at PEP/PETRA energies, which is hardly compatible with the JETSET prediction. A comparison at all three energies suggests that the production of $\eta^{\prime}$ and $\Omega^{-}$in data was overestimated and the Monte Carlo prediction was correct. Both models, JETSET and HERwig, describe the ratios of the hadron multiplicities per event for all the other hadrons.

The trend that the increase in baryon production was stronger than for mesons, which is observed comparing $10 \mathrm{GeV}$ with $35 \mathrm{GeV}$ data [62], is not seen with LEP data compared to PEP/PETRA data.

The scalar and tensor mesons show a different behaviour. Their production is large at LEP. However, the $\mathrm{K}_{2}^{*}$ multiplicity has a large error; for the $f_{0}$ the low ratios are due to a low rate measured at $10 \mathrm{GeV}$. This implies that the $L=1$ hadrons are more numerously produced than one had previously expected. Their production and decay has influence on lighter hadrons, e.g., softening their momentum spectrum. 


\section{Chapter 6}

\section{Momentum Spectra}

In this chapter, the production of particles measured by the four LEP experiments is discussed in more detail. In Table 5.1 the number of particles per event is given with their total error and, if not done by the collaborations themselves, extrapolated to the full momentum range. This has allowed a fast overview and an easy comparison of the experimental results.

Particles analyses are grouped into sections according to the experimental procedure for the signal extraction. The table at the beginning of each section briefly lists the particles with: their masses; their decay lengths or widths; the decay mode with its branching ratio used for the investigation. Also included are the experiments, from which measurements have been published. The experimental results are presented with the statistical and systematic errors given separately (if provided in the published papers). When a third error is given, it is due to the extrapolation in $x_{p}$; otherwise the error source is indicated. Sometimes the result is given in a limited momentum range, or multiplied by a branching ratio etc., details are stated where necessary. The explanation for the signal extraction is more detailed for the particles discussed first: particle identification with $\mathrm{d} E / \mathrm{d} x$ etc.

The momentum spectra are compared with the Monte Carlo models JETSET and HERWIG, tuned to ALEPH data as described in Section 3.4 with the parameters given in Tables 3.3 and 3.4. Most spectra in publications are given as the differential cross section $\left(1 / \sigma_{\text {tot }}\right) \mathrm{d} \sigma^{\mathrm{h}} / \mathrm{dx}_{\mathrm{p}}\left(x_{p}=p_{\text {hadron }} / p_{\text {beam }}\right)$, some are given in $\left(1 / \beta \sigma_{\text {tot }}\right) \mathrm{d} \sigma^{\mathrm{h}} / \mathrm{dx}_{\mathrm{E}}$ or $\left(1 / \sigma_{\text {tot }}\right) \mathrm{d} \sigma^{\mathrm{h}} / \mathrm{dx}_{\mathrm{E}}\left(x_{E}=E_{\text {hadron }} / E_{\text {beam }}, \beta=v / c\right)$. The particle spectra of all experiments were recalculated to the differential cross section in $x_{p}$ to allow comparison. $\mathrm{d} \sigma^{\mathrm{h}} / \mathrm{dx}_{\mathrm{p}}$ is equivalent to $\beta \mathrm{d} \sigma^{\mathrm{h}} / \mathrm{d} \mathrm{x}_{\mathrm{E}}$. At PEP/PETRA energies the experiments used $(s / \beta) \mathrm{d} \sigma^{\mathrm{h}} / \mathrm{d} \mathrm{x}_{\mathrm{E}}$, because this was expected to be independent from the centre-of-mass energy $\sqrt{s}$. The deviation seen by the experiments showed the scale breaking by gluon radiation (See $[62,105]$ for a summary.). For a few particles, the experiments provided no tables and we show the published spectra (in $x_{E}$ ). For particles with large production probability at small $x_{p}$, we also give the differential cross section $\left(1 / \sigma_{\text {tot }}\right) \mathrm{d} \sigma^{\mathrm{h}} / \mathrm{d} \xi\left(\xi=\ln \left(1 / x_{p}\right)\right)$.

The differential distributions in $x_{p}$ and $\xi$ are shown for data, included are the total errors. When the statistical and systematic errors are given separately in the tables of the publications, they are added in quadrature; some experiments quote additional 
systematic errors for the normalization, typically $2-4 \%$, which were added in quadrature as well. The data points for the $x_{p}$ distributions are placed in $x_{p}$, where the values of the predicted function (here: JETSET) ought to appear, i.e., is equal to its mean over the bin, as recommended in Ref. [107]. In addition, we indicate the width of the bins, which are different from experiment to experiment, by a horizontal bar. For the $\xi$ distributions, which are not monotonous in the measured range, the data points are put into the centre of the bin.

\subsection{Stable Particles}

\section{Extraction of $\pi^{ \pm}, \mathbf{K}^{ \pm}$, and $(\mathrm{p}, \overline{\mathrm{p}})$ Signals}

\begin{tabular}{|c|c|c|c|c|c|}
\hline \multicolumn{2}{|c|}{ Particle } & $\begin{array}{c}\text { Mass } \\
{\left[\mathrm{MeV} / \mathrm{c}^{2}\right]}\end{array}$ & $\begin{array}{l}c \tau \\
{[\mathrm{m}]}\end{array}$ & Decay mode & $\begin{array}{l}\text { BR } \\
{[\%]}\end{array}$ \\
\hline all charged & ADLO & & & & \\
\hline$\pi^{ \pm}$ & $\mathrm{AO}$ & 139.6 & 7.8 & "stable" & \\
\hline $\mathrm{K}^{ \pm}$ & $\mathrm{ADO}$ & 493.7 & 3.7 & "stable" & \\
\hline $\mathrm{p}$ & $\mathrm{ADO}$ & 938.3 & 0.0 & stable & \\
\hline & & Multiplici & $\bar{y} / \mathrm{Ev}$ & & $\overline{\text { Ref. }}$ \\
\hline all charged & ALEPH & $20.85 \pm 0$. & $\overline{2 \pm 1}$ & & 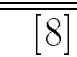 \\
\hline & DELPHI & $20.71 \pm 0$. & & & {$[9]$} \\
\hline & L3 & $20.70 \pm 0$. & & & {$[10]$} \\
\hline & OPAL & $21.40 \pm 0$. & $2 \pm 1$ & .43 & [11] \\
\hline$\pi^{ \pm}$ & ALEPH & spectrum & & & {$[12]$} \\
\hline & OPAL & $17.05 \pm 0$. & & & {$[13]$} \\
\hline$K^{ \pm}$ & ALEPH & spectrum & & & {$[12]$} \\
\hline & DELPHI & $2.26 \pm 0.0$ & \pm 0 & $6 \pm 0.09$ & {$[17]$} \\
\hline & OPAL & $2.42 \pm 0.1$ & & & {$[13]$} \\
\hline $\mathrm{p}$ & ALEPH & spectrum & & & {$[12]$} \\
\hline & DELPHI & $1.07 \pm 0.0$ & \pm 0 & $5 \pm 0.13$ & {$[17]$} \\
\hline & OPAL & $0.92 \pm 0.1$ & & & {$[13]$} \\
\hline
\end{tabular}

Table 6.1: Properties of stable particles and their measured average multiplicities.

Three experiments have measured the productions of the three stable charged hadrons, which leave their tracks in the tracking chambers: $\pi^{ \pm}, K^{ \pm}$, and $(p, \bar{p})$. These particles with decay length in the laboratory frame, large compared to or compatible with the detector dimensions, can be identified by a simultaneous measurement of their momentum and the specific energy loss $\mathrm{d} E / \mathrm{d} x$; a method which is exploited both by the ALEPH and the OPAL experiment. The DELPHI collaboration uses its two RICH counters for additional particle identification (Figure 4.1). 
For the extraction of the fraction $f_{i}$ of particle $i$ with the specific energy loss method, in each momentum interval the distribution of $\mathrm{d} E / \mathrm{d} x$ is given for each particle $i$ by the probability density

$$
g\left(\mathrm{~d} E / \mathrm{d} x, f_{i}\right)=\sum_{i} \frac{f_{i}}{\sqrt{2 \pi} \sigma_{i}} \exp \left(\frac{-\left(\mathrm{d} E / \mathrm{d} x-\mu_{i}(p)\right)^{2}}{2 \sigma_{i}^{2}}\right) .
$$

$\mu_{i}$ is the expected $\mathrm{d} E / \mathrm{d} x$ for particle type $i$ and $f_{i}$ is the fraction of the particle type $\mathrm{e}^{ \pm}$, $\pi^{ \pm}, \mathrm{K}^{ \pm}$, and $(\mathrm{p}, \overline{\mathrm{p}})$. In each momentum interval the $\mathrm{d} E / \mathrm{d} x$ distribution can be composed as a sum of these four Gaussian functions (Figure 6.1). With the additional constraint $\sum_{i} f_{i}=1$, one obtains the fraction by maximizing the function

$$
\mathcal{L}=\frac{\mathrm{e}^{-\phi} \phi^{N}}{N !} \prod_{j=1}^{N} g_{j}\left(\mathrm{~d} E / \mathrm{d} x, f_{i}\right)
$$

with a Poisson factor in front. It represents the probability of obtaining a sample of size $N$ from a distribution of mean $\phi$. In the fit $\mathrm{d} E / \mathrm{d} x, \mu_{i}$, and $\sigma_{i}$ are functions of the number of samplings and track length; the Gaussian used for the probability density may be replaced by a sum of two Gaussians.

The DELPHI collaboration, in their analysis of RICH measurements, has defined its probability density $l$ (instead of $g$ ) correspondingly:

$$
l\left(\theta, f_{i}\right)=\frac{1}{C}\left(b+\sum_{i} \frac{f_{i}}{\delta \theta^{i}} \exp \left(\frac{-\left(\theta-\theta^{i}\right)^{2}}{2\left(\delta \theta^{i}\right)^{2}}\right)\right),
$$

with the constraint $\sum_{i} f_{i}=1\left(i=\pi^{ \pm}, \mathrm{K}^{ \pm}\right.$, and $(\mathrm{p}, \overline{\mathrm{p}})$; electrons and muons are not treated separately, but are taken as pions). $\theta$ and $\theta^{i}$ are the measured and expected Cherenkov angles with error $\delta \theta^{i}$. The constant $b$ for background and the normalization $C$ are additional free parameters in the fit (Figure 6.2).

Both procedures do not distinguish between muons and pions. Studies using simulated events show that a correction for this combined treatment is appropriate and amounts to $2 \%$ to $5 \%$. A correction of the same order is applied for the fraction of these 'stable' hadrons, which decay inside the detector. The decay products of particles with lifetimes shorter than $10^{-9} \mathrm{~s}$, such as $\mathrm{K}_{\mathrm{s}}^{0},(\Lambda, \bar{\Lambda})$ are included in the spectra. Additional particles generated by nuclear interactions inside the detector material (about 10\%) must be corrected for. For the $(p, \bar{p})$ spectra, it is appropriate to use anti-protons only, because protons may emerge from nuclear interactions in the detector material. From this, the largest error arises for charged pions, comparable with the uncertainty of the simulation of the number of $\mathrm{d} E / \mathrm{d} x$ samplings. For kaons and protons the dominant error comes from the imperfect knowledge of the expected $\mathrm{d} E / \mathrm{d} x$ and its shape. For the fit with the information of the RICH, the uncertainty on the efficiency, which is mainly determined by the number of photo-electrons of the Cherenkov rings, is largest.

\section{Momentum Spectra}

In Figure 6.3 and 6.4 the results of the ALEPH, DELPHI and OPAL experiments are compared to the model predictions of JETSET and HERWIG. The coherent shower mod- 

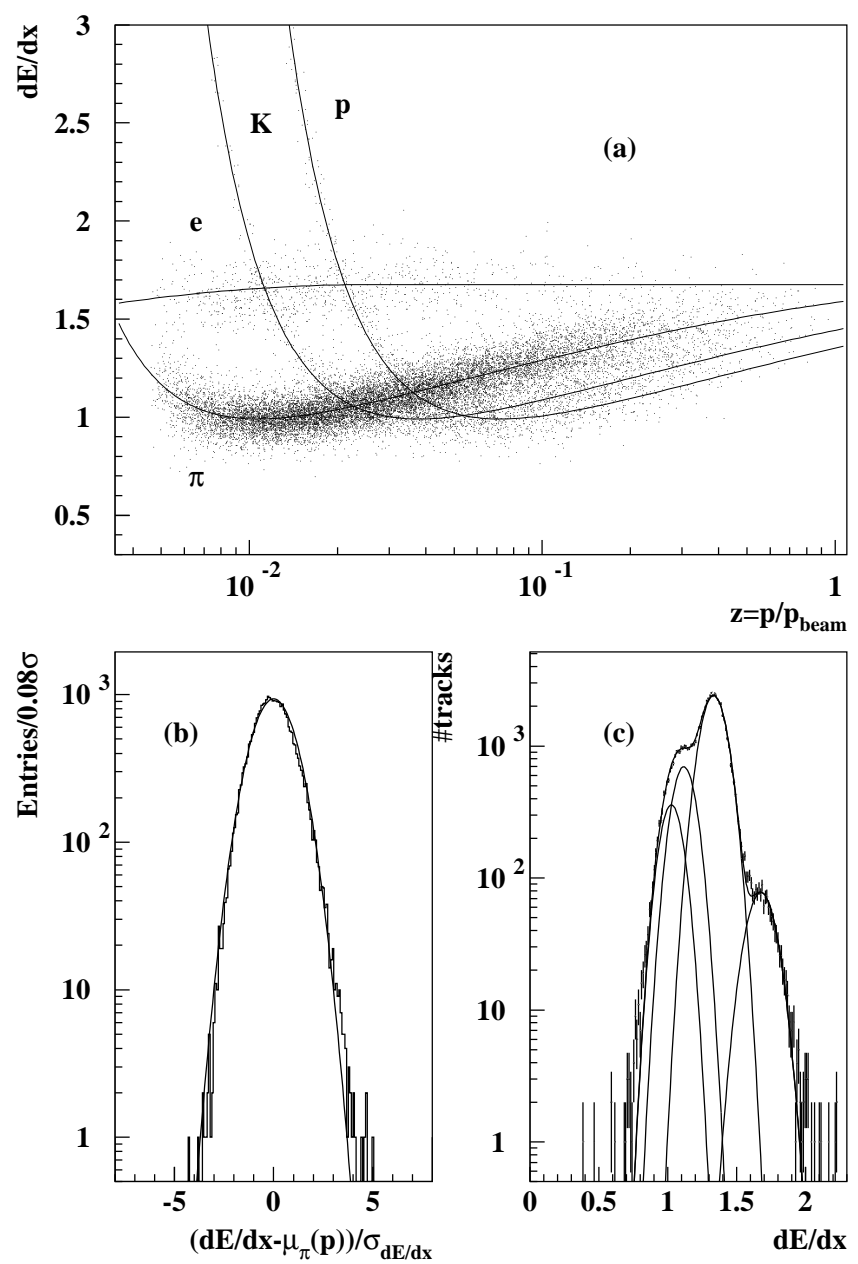

Figure 6.1: Energy loss as function of momentum $x_{p}=p / p_{\text {beam }}$. a) Data measured in the ALEPH detector are compared with the expectation for $e, \pi, K$, and p. b) Energy loss (measurement - expectation for pions) of minimum ionizing pions $(0.40<p<$ $0.55 \mathrm{GeV} / \mathrm{c}$. c) Likelihood fit to data in the momentum range $0.12<x_{p}<0.13$ [12].

els, such as the HERWIG cluster model and JETSET string fragmentation model, both shown in the figures reproduce the measurements. Already at LEP startup, when the accumulated event statistics was still low, the collaborations could show the failure of incoherent models, e.g., those using independent fragmentation. The coherent models, such as JETSET and HERWIG, show a maximum in the $\xi$ distribution with a Gaussian shape as predicted from QCD and measured by the experiments.

For particle identification discussed above, the two different measurement techniques are complementary: the DELPHI points fill the momentum gap not measurable with the specific energy loss method used by ALEPH and OPAL, where the kaon and pion energy loss is similar.

The measured charged pion spectra agree nicely. They coincide with both model predictions. This is not surprising, because the models have been tuned to the charged particle inclusive momentum distribution. With most particles being pions, the agree- 


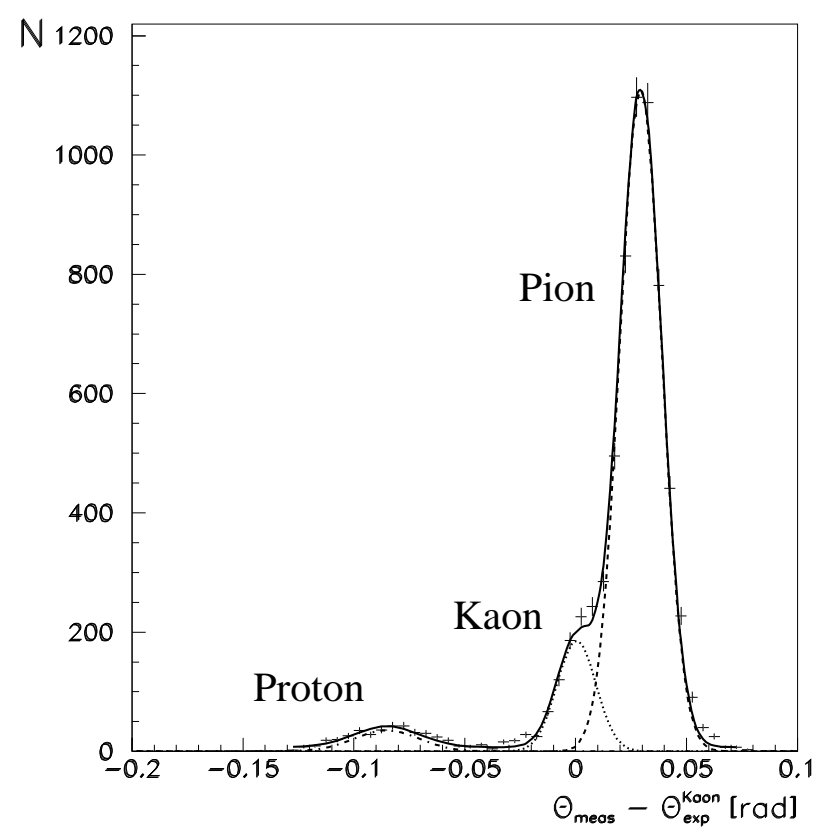

Figure 6.2: Cherenkov angle (measurement - expectation for kaons) measured with the DELPHI liquid radiator with the fit of three Gaussians and a flat background [17].

ment reflects the good quality in the fitting of the model tuning. Nevertheless, small discrepancies are observed for very small momenta, clearly seen in the $\xi$-distributions of Figures 6.4. The charged kaon spectra agree within the errors for high momenta. For kaons with momenta from $\xi=1$ to the maximum of the $\xi$ distribution, no model succeeds in reproducing the data. This may hint to an imperfect description of kaon decays of B-hadrons. For protons, ALEPH measures a harder spectrum than OPAL. Especially at high momenta the difference is striking and certainly needs some clarification by measurements in this range from the other LEP experiments, DELPHI and L3. The models, both show a harder spectrum. This can be easily seen in Figure 6.5 of a study done by OPAL, when plotting the fraction $f_{i}$ of all stable particles. We see a drop at high momenta for protons, which was stronger than the original models would predict. A solution might be a suppression of first rank, leading baryons except in c- and b-jets; this can be understood in terms of diquark suppression at small proper times [108], which lead to the implementation of a new free parameter into the JETSET generator. The question, whether excited baryons could help, can be asked; however, measurements would be needed.

The transverse momentum of all charged particles with respect to the sphericity axis, in and out of the event plane $\left(p_{t}^{\text {in }}\right.$ and $\left.p_{t}^{\text {out }}\right)$, has been measured. These distributions (Figure 3.10) are sensitive to gluon radiation. The $p_{t}^{\text {out }}$ distribution from data is harder than the predictions and indicates missing higher order corrections in the simulation, needed for a proper description of the data. 

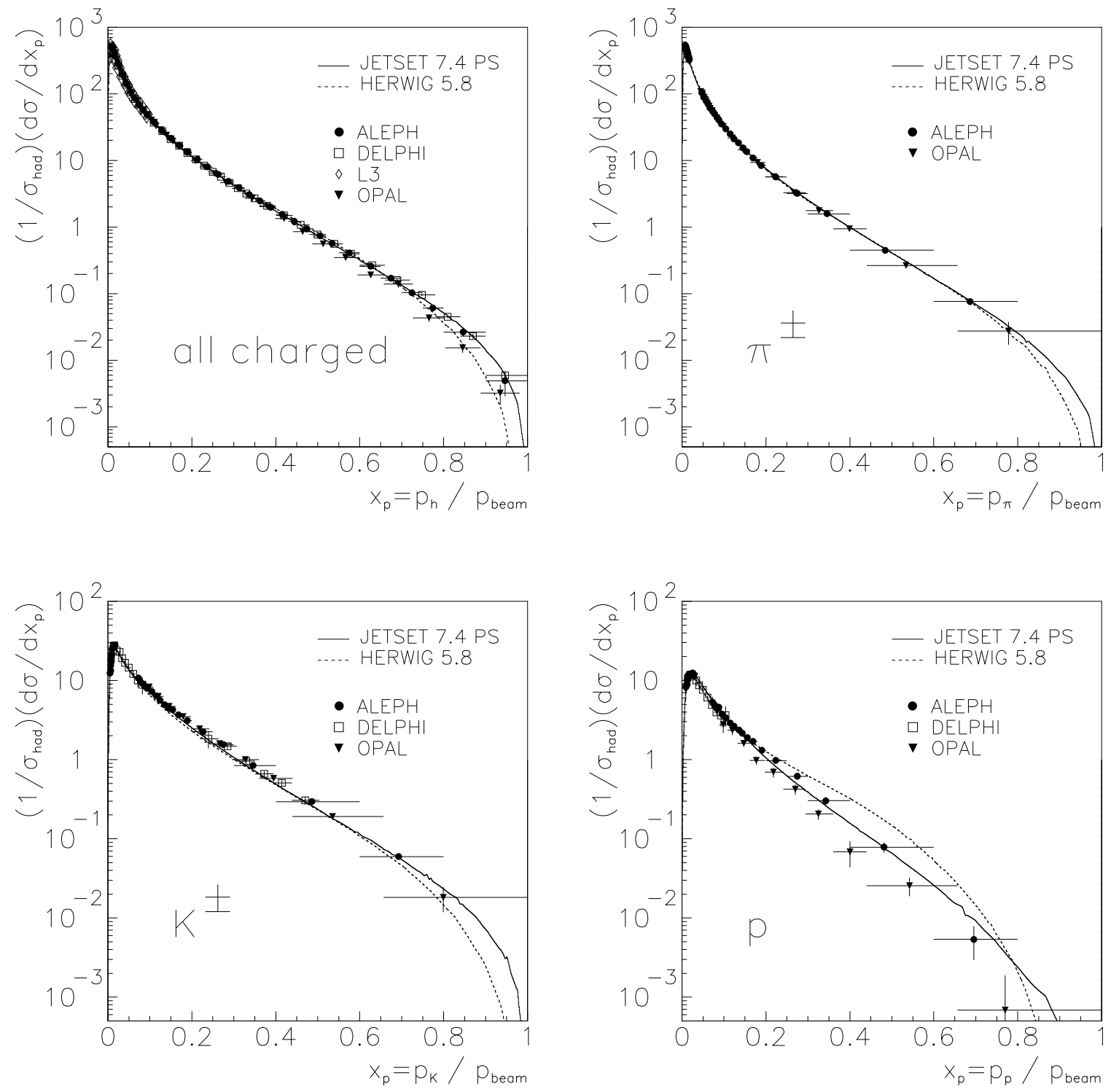

Figure 6.3: Momentum spectra in $x_{p}$ for charged particles, $\pi^{ \pm}, K^{ \pm}$, and $(p, \bar{p}) ; x_{p}=$ $p_{\text {hadron }} / p_{\text {beam }}$. 

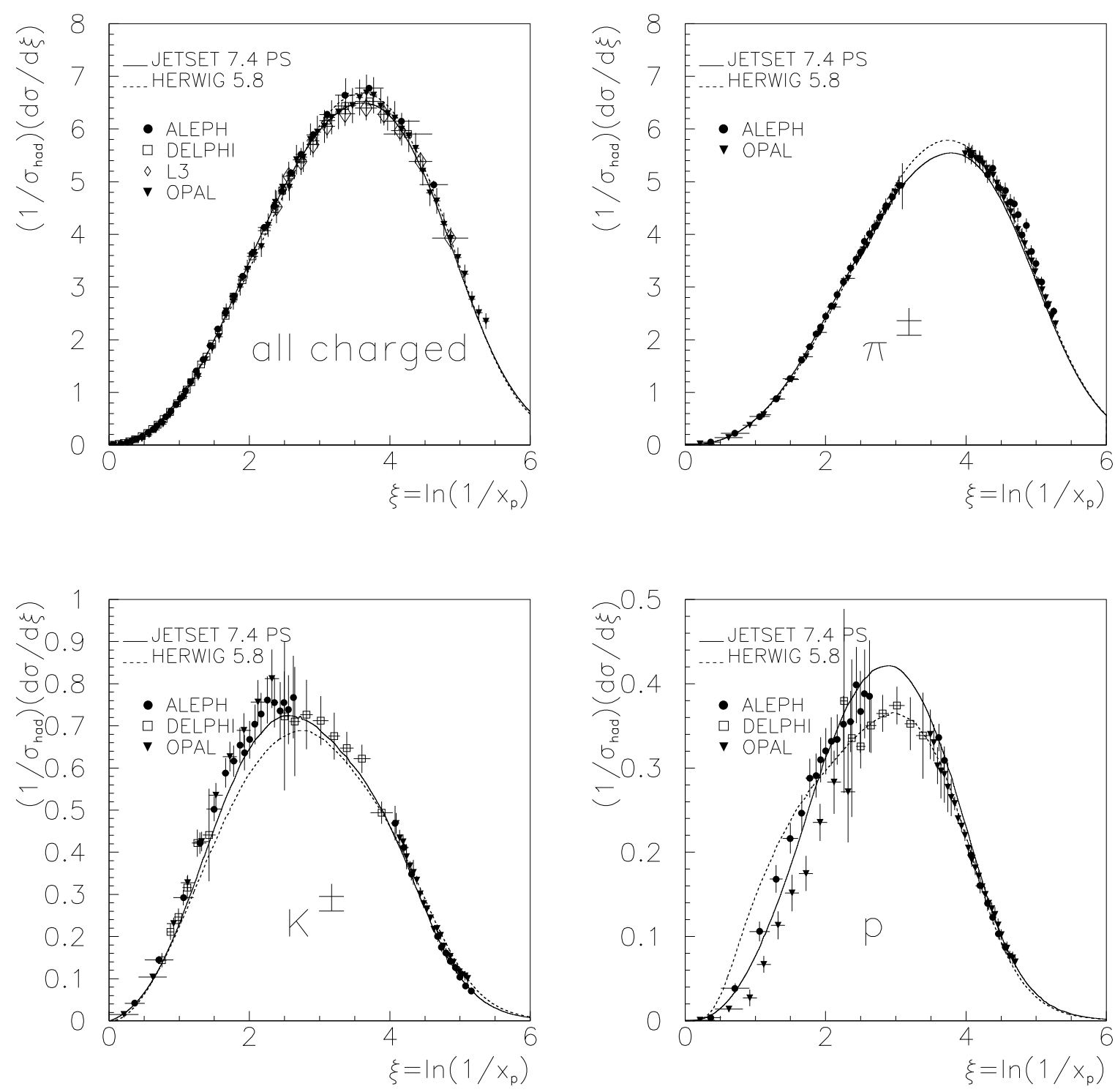

Figure 6.4: Momentum spectra in $\xi$ for charged particles, $\pi^{ \pm}, K^{ \pm}$, and $(p, \bar{p}) ; \xi=$ $\ln \left(1 / x_{p}\right)=\ln (\sqrt{s} / 2 p)$. 


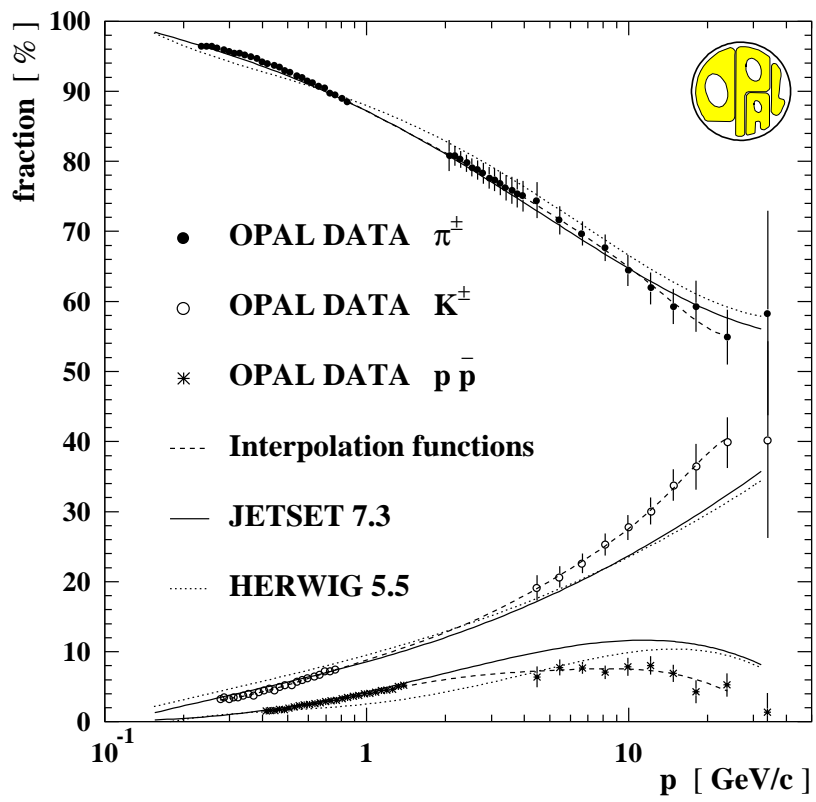

Figure 6.5: Fraction of pion, kaon, and proton of all charged particles in OPAL data showing the suppression of baryons at high momenta [13]. 


\section{2 $\mathrm{V}^{0}$ Particles}

\section{Extraction of $\mathrm{K}_{\mathrm{s}}^{0}$ and $\Lambda$ Signals}

\begin{tabular}{|rr|r|r|l|l|}
\hline \multicolumn{2}{|c|}{ Particle } & $\begin{array}{c}\text { Mass } \\
{\left[\mathrm{MeV} / c^{2}\right]}\end{array}$ & $\begin{array}{c}c \tau \\
{[\mathrm{cm}]}\end{array}$ & Decay mode & $\begin{array}{c}\text { BR } \\
{[\%]}\end{array}$ \\
\hline \hline $\mathrm{K}^{0}\left(\mathrm{~K}_{\mathrm{s}}^{0}+\mathrm{K}_{\mathrm{l}}^{0}\right)$ & ADLO & 497.7 & 2.7 & $\mathrm{~K}_{\mathrm{s}}^{0} \rightarrow \pi^{ \pm} \pi^{\mp}$ & 68.6 \\
\hline$\Lambda$ & ADLO & 1115.7 & 7.9 & $\mathrm{p} \pi^{-}$ & 63.9 \\
\hline \hline & & Multiplicity/Event & Ref. \\
\hline \hline $\mathrm{K}^{0}$ & ALEPH & $2.061 \pm 0.047$ & {$[18]$} \\
& DELPHI & $1.962 \pm 0.022 \pm 0.056$ & {$[19]$} \\
& L3 & $2.04 \pm 0.02 \pm 0.14$ & {$[16]$} \\
& OPAL & $1.99 \pm 0.01 \pm 0.04$ & {$[20]$} \\
\hline$\Lambda$ & ALEPH & $0.386 \pm 0.016$ & {$[18]$} \\
& DELPHI & $0.357 \pm 0.003 \pm 0.017$ & {$[32]$} \\
& L3 & $0.37 \pm 0.01 \pm 0.04$ & {$[16]$} \\
& OPAL & $0.363 \pm 0.002 \pm 0.022$ & {$[33]$} \\
\hline
\end{tabular}

Table 6.2: Properties of $V^{0}$ particles and their measured average multiplicities.

All four LEP experiments have measured the momentum spectra of two $V^{0}$ particles; the $\mathrm{K}_{\mathrm{s}}^{0}$ and the $\Lambda$. These particles are easily reconstructed by their distinct signature: a decay into two charged particles, seen in the detector with the decay vertex separated from the $\mathrm{e}^{+} \mathrm{e}^{-}$collision point (if the $\mathrm{V}^{0}$ 's lifetime is long enough). The selection of $\mathrm{K}_{\mathrm{s}}^{0}$ and $\Lambda$ candidates proceeds via a fit of a secondary vertex of all oppositely charged particle pairs. If this vertex is well separated from the primary vertex and the reconstructed mass is compatible with the mass of the particle studied, a candidate is formed. For the $\Lambda$ (or $\bar{\Lambda}$ ) the choice of which daughter is the proton (or anti-proton) must be made; the particle with higher momentum is usually chosen. Additional information is provided by the $\mathrm{d} E / \mathrm{d} x$ measurement. Further background rejection is obtained by a cut on the decay angle in the $\mathrm{V}^{0}$ 's rest frame and by a cut on the angle between the flight direction reconstructed from the momentum of the $V^{0}$ and the direction given by the primary and secondary vertex. The latter cut uses the assumption that the $\mathrm{V}^{0}$ 's are produced at the collision point. A kinematical $V^{0}$ fit, constrained by the mass hypothesis and the collision point in the transverse plane, considerably further reduces the background. The analysis has also to deal with the fact that a track pair can fulfill the criteria of both, the $\mathrm{K}_{\mathrm{s}}^{0}$ and $\Lambda$, when no particle identification is possible. However, pure kinematics using transverse and longitudinal momenta of positively and negatively charged particles with respect to $\mathrm{V}^{0}$ momentum allows a good separation: Figure 6.6 shows the Armenteros-plot [109], where the transverse component $p_{t}$ of a daughter track with respect to the $\mathrm{V}^{0}$ direction is plotted versus the asymmetry $\alpha=\left(p_{l}^{+}-p_{l}^{-}\right) /\left(p_{l}^{+}+p_{l}^{-}\right)$. Converted photons may look like $\mathrm{V}^{0}$ 's when they convert to an $\mathrm{e}^{+} \mathrm{e}^{-}$pair, but are rejected by the requirement that the 
invariant mass of the $\mathrm{V}^{0}$ should exceed some value (typically $30 \mathrm{MeV} / \mathrm{c}^{2}$ ). This possibility of photon reconstruction via conversion in the detector material has the advantage of good momentum resolution compared to the calorimetric measurement, and is extensively used for the $\pi^{0}$ analysis described in the next section. Another ambiguity arises in a few percent of the cases, when two vertices are reconstructed from the daughters. Investigations with simulated events show that the ambiguities can be resolved by checking whether hits are reconstructed on the tracks between the two reconstructed vertices; if so, the vertex with smaller distance to the collision point is taken. Other possibilities are to take the selection with the smallest $\chi^{2}$ of the vertex fit, or perform a fit in the transverse plane and to choose the smallest distance between the decay tracks along the beam line.

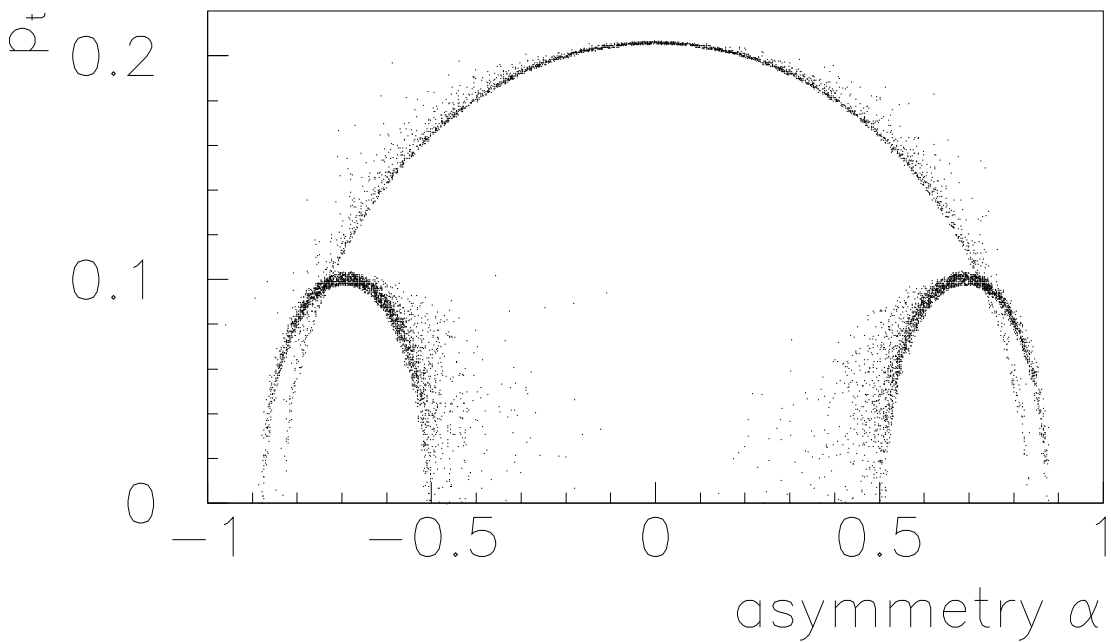

Figure 6.6: Separation of $\Lambda$ and $\mathrm{K}_{\mathrm{s}}^{0}$ in the Armenteros-plot. The transverse component $p_{t}$ is drawn versus the asymmetry $\alpha=\left(p_{l}^{+}-p_{l}^{-}\right) /\left(p_{l}^{+}+p_{l}^{-}\right)$showing the band for $\mathrm{K}_{\mathrm{s}}^{0}$ and the two bands for $\Lambda$ and $\bar{\Lambda}[109]$.

\section{Momentum Spectra}

All experiments agree in their findings that the measured momentum spectrum for neutral kaons is harder than predicted by the models (Figures 6.7). Even after model tuning no perfect description can be obtained. An improvement is achieved when tensor meson production is enabled and the strangeness suppression is adapted, such that the rate of the vector meson $\mathrm{K}^{*}$ agreed with the measured values. The remaining difference may be due to heavy flavour decays. Not all production cross sections and branching ratios are known and implemented in the model.

For the $\Lambda$ production, Figures 6.7 show that the LEP collaborations agree in their measurements. JETSET has a yield which agrees for high momenta, is slightly high around the maximum in $\xi$ and agrees again for lower momentum. This is an improvement to earlier model tunings, now that leading baryons are suppressed. The failure of an exact description is the fact of a compromise for the proton and $\Lambda$ spectra. HeRWIG is too high 

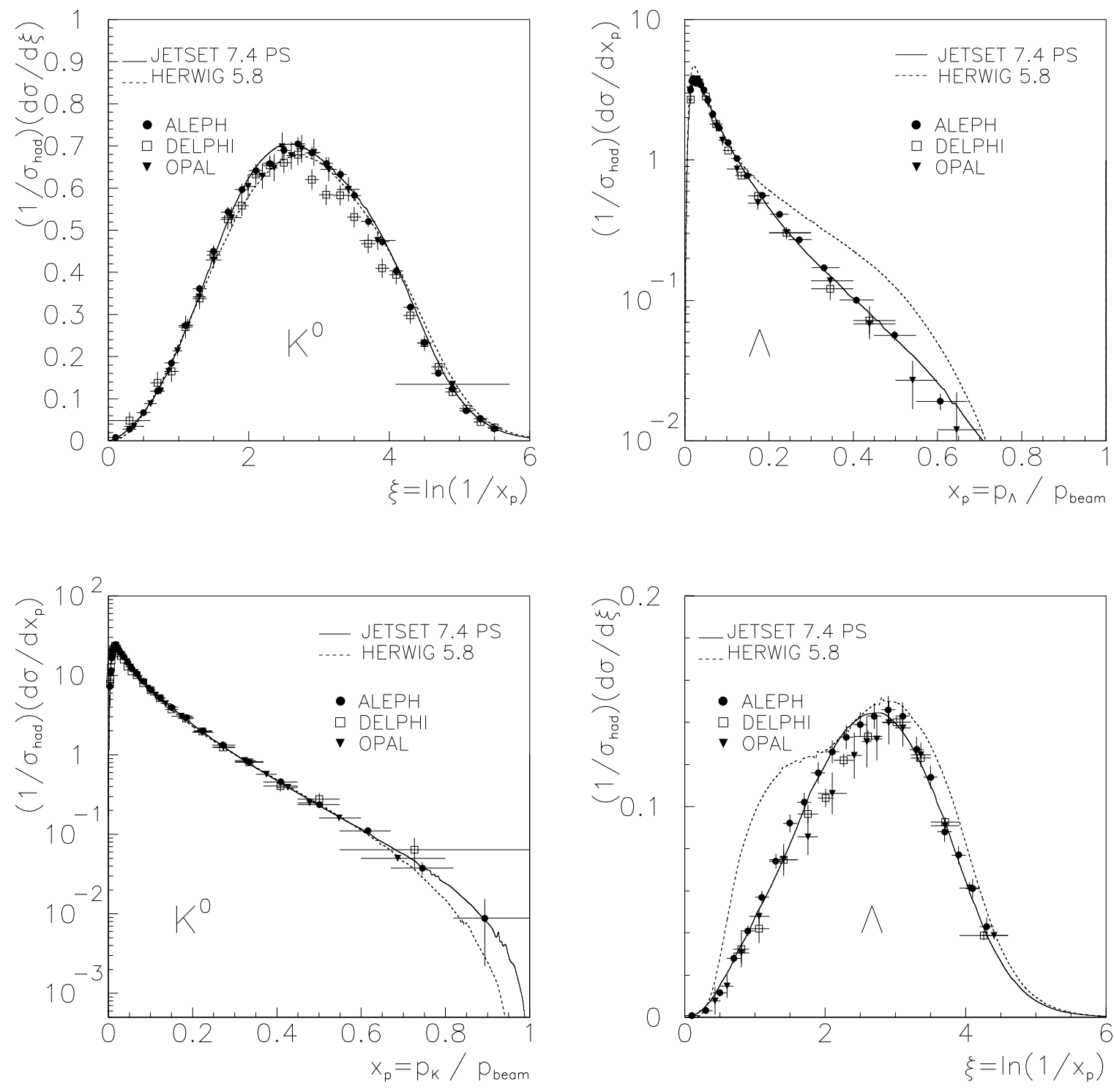

Figure 6.7: Momentum spectra for $\mathrm{K}_{\mathrm{s}}^{0}$ and $\Lambda$.

at all momenta and shows some structure; the much too high rate at high momenta in HERWIG is caused by too many $\Lambda$ containing primary quarks. The shoulder seen at small $\xi$ in the $\xi$-spectrum, generated with the HerwIG Monte Carlo, indicates a too high $\Xi$ and $\Sigma$ production. Direct hyperon production measurements support this point of view, the rates being described better by JETSET than by HERWIG.

The ALEPH collaboration has also measured the transverse momentum with respect to the thrust axis; see Figure 6.8. The thrust axis was determined using all charged and neutral particles measured in the detector. The axis was corrected for imperfections of the detector, i.e., corrected for the loss of final state particles except neutrinos. The transverse 

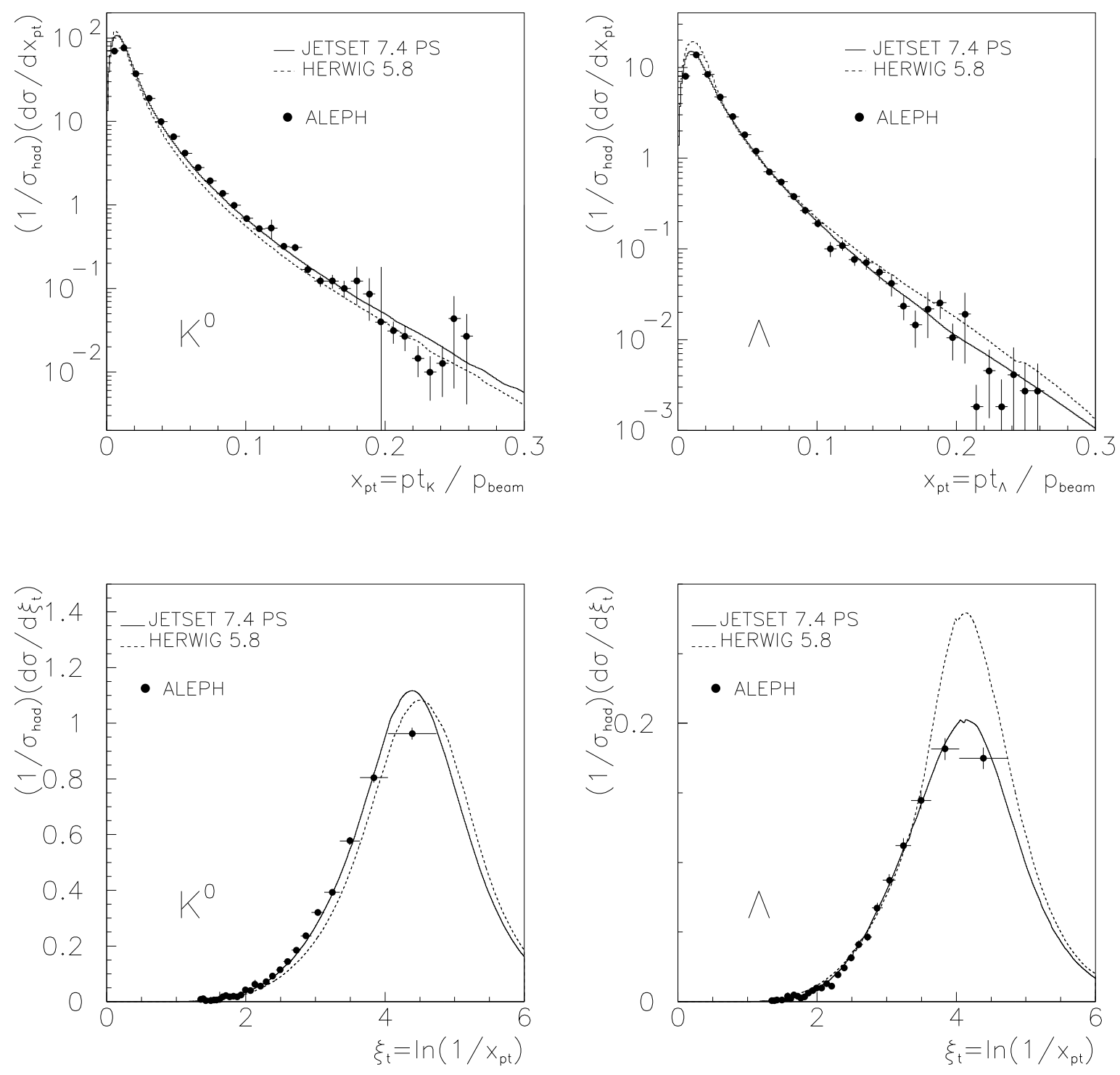

Figure 6.8: Transverse momentum spectra for $\mathrm{K}_{\mathrm{s}}^{0}$ and $\Lambda$. The transverse momentum $p_{t}$ is measured with respect to the thrust axis, which is calculated from charged and neutral particles.

momentum distribution for the neutral kaon is underestimated by both JETSET and HERWIG; especially in the range 0.05 to 0.1 in $x_{p t}=p_{t, \text { hadron }} / p_{\text {beam }}$. For the $\Lambda$ spectrum, JETSET describes the data, while the HERWIG model overestimates the production of the baryons with low transverse momentum. 


\subsection{Particles Decaying to Photons}

\section{Extraction of $\pi^{0}, \eta$, and $\eta^{\prime}$ Signals}

\begin{tabular}{|c|c|c|c|c|c|}
\hline \multicolumn{2}{|c|}{$\begin{array}{r}\text { Particle } \\
\text { Exp. }\end{array}$} & $\begin{array}{c}\text { Mass } \\
{\left[\mathrm{MeV} / \mathrm{c}^{2}\right]}\end{array}$ & $\begin{array}{c}\text { Width }(c \tau) \\
{\left[\mathrm{MeV} / c^{2}(\mathrm{~nm})\right]}\end{array}$ & Decay mode & $\begin{array}{l}\mathrm{BR} \\
{[\%]}\end{array}$ \\
\hline 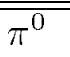 & $\overline{\mathrm{ADL}}$ & 135.0 & (25.1) & $\gamma \gamma$ & 98.8 \\
\hline$\eta$ & $\mathrm{AL}$ & 547.5 & 0.001 & $\gamma \gamma$ & 38.8 \\
\hline \multirow[t]{2}{*}{$\eta^{\prime}$} & $\overline{\mathrm{AL}}$ & 957.8 & 0.201 & $\begin{aligned} \pi^{ \pm} \pi^{\mp} \eta & \\
& \hookrightarrow \gamma \gamma\end{aligned}$ & $\begin{array}{l}43.7 \\
38.8 \\
\end{array}$ \\
\hline & & \multicolumn{3}{|c|}{ Multiplicity/Event } & Ref. \\
\hline & ALEPH & \multicolumn{3}{|c|}{$9.63 \pm 0.13 \pm 0.66 \pm 0.05$} & {$[14]$} \\
\hline & DELPHI & \multicolumn{3}{|c|}{$9.2 \pm 0.2 \pm 1.0$} & {$[15]$} \\
\hline & L3 & \multicolumn{3}{|c|}{$9.18 \pm 0.03 \pm 0.73$} & {$[16]$} \\
\hline \multirow[t]{2}{*}{$\eta$} & ALEPH & \multicolumn{3}{|c|}{$0.282 \pm 0.015 \pm 0.016\left(x_{E}>0.1\right)$} & [21] \\
\hline & L3 & \multicolumn{3}{|c|}{$0.91 \pm 0.02 \pm 0.011$} & {$[16]$} \\
\hline \multirow[t]{2}{*}{$\eta^{\prime}$} & ALEPH & \multicolumn{3}{|c|}{$0.064 \pm 0.013 \pm 0.005\left(x_{E}>0.1\right)$} & [21] \\
\hline & L3 & \multicolumn{3}{|c|}{$0.265 \pm 0.028 \pm 0.054$} & {$[22]$} \\
\hline
\end{tabular}

Table 6.3: Properties of particles decaying to photons and their measured average multiplicities.

About one third of the centre-of-mass energy in $\mathrm{e}^{+} \mathrm{e}^{-}$annihilation is emitted in the form of neutral particles. The energy of most of them is detected in the electromagnetic calorimeter: the majority of the stable particles seen in the detector are charged pions. To first approximation, the number of $\pi^{+}, \pi^{0}$, and $\pi^{-}$are the same as predicted by isospin symmetry. We therefore expect about 20 photons in a hadronic event. Most photons are decay products of the neutral pions produced. Decays of the $\eta$ and decays of the $\eta^{\prime}$ via $\eta$ and two charged pions, give further contributions. The decay of the $\eta^{\prime}$ to $\rho^{0} \gamma(\mathrm{BR}=30 \%)$ has not yet been seen.

Photons are detected by their energy deposition in the electromagnetic calorimeter with the additional requirement that no track points to the shower. The best energy resolution of the four experiments is achieved by the L3 collaboration. With their BGO calorimeter they measure photons down to $50 \mathrm{MeV}$. The other experiments measure photons typically down to $1 \mathrm{GeV}$. The angular resolution of a calorimeter is worse than the tracking chamber resolution. This implies an upper limit on the momentum for detection of a $\pi^{0}$ : for high momentum $\pi^{0}$ 's the showers of the two photons overlap and are reconstructed in the calorimeter as one cluster. Special pattern recognition programs, looking for subclusters, can detect these merged $\pi^{0}$ 's up to $15 \mathrm{GeV}$ or $25 \mathrm{GeV}$, depending on the granularity of the calorimeter and the isolation from other calorimetric activity.

The detection range for $\pi^{0}$ s $\mathrm{s}$ is extended using photons, which have converted to an $\mathrm{e}^{+} \mathrm{e}^{-}$-pair in the detector material. The two tracks are then reconstructed in the tracking chambers just like a $V^{0}$. The fact that one photon of the $\pi^{0}$ has converted often leaves the 
other photon as an isolated shower in the calorimeter; high momentum $\pi^{0}$ 's can be seen in this way. This method was has been used by ALEPH to reconstruct $\pi^{0}$ 's up to $x_{p}=1$. Converted photons, however, allow an extension to lower energies as well. The threshold for charged tracks is lower than for the calorimetric showers (except for L3); two tracks with $p>150 \mathrm{MeV} / c$ give a lower detection limit for photons of $300 \mathrm{MeV} / c$. The DELPHI collaboration has pushed the limit even further. The fact that a converted photon is a $V^{0}$ with an invariant mass of zero, is used for the photon finding: instead of fitting a secondary vertex, the reconstruction procedure analyses single tracks. The point where the tangent of the reconstructed track is pointing back to the beam line is taken as conversion point, when this point is separated from the collision point. If two oppositely charged tracks are consistent with having a common conversion point, a conversion candidate is formed. For very low momenta one of the two conversion electrons (positrons) may be lost in the beam pipe or its track may not have sufficient points for reconstruction due to asymmetric pair production. A photon conversion candidate is formed from one track only, when its conversion point is in the detector material.

The $\pi^{0}$ momentum spectrum can be measured over most of the momentum range with the three methods of $\pi^{0}$ reconstruction, and comparisons can be used as consistency check for the signal extraction.

The $\eta$, which has a larger mass than the $\pi^{0}$, is measured by reconstructing both photons in the calorimeter. The opening angle of the two decay photons is larger, because the $\eta$ has a higher mass than the $\pi^{0}$. Photons which show up also in $\pi^{0}$ candidates are not used for the $\eta$ analysis. By combining the $\eta$ with two oppositely charged particles an $\eta^{\prime}$ signal is seen. ALEPH measures the $\eta$ in the decay $\eta \rightarrow \gamma \gamma$ and, as efficiency cross check in its $\omega$ analysis, $\eta \rightarrow \pi^{+} \pi^{-} \pi^{0}$; the production rates extracted in these two decay modes agree.

The extraction of the signals is performed fitting the mass spectra with an analytical function for the background and a Gaussian for the signal, with the width and shape determined by the detector resolution. In the case of the $\eta$, care has to be taken to exclude the $\omega$ reflection. The decay $\omega \rightarrow \pi^{0} \gamma$ contributes to the $\gamma \gamma$ mass around $750 \mathrm{MeV} / c^{2}$, if the $\pi^{0}$ is not resolved as two photons.

\section{Momentum Spectra}

The inclusive momentum distributions for $\pi^{0}, \eta$, and $\eta^{\prime}$ are shown in Figure 6.9 ; for the $\pi^{0}$ the $\xi$ distribution is given, because the measurement is accurately extracted down to very low momenta ( $140 \mathrm{MeV} / \mathrm{c}$ for the L3 experiment). The L3 measurement obtains different results for the $\pi^{0}$ production, depending whether JETSET or HERWIG is used for efficiency and acceptance corrections. The average of the two results is taken and half of the difference is added as an additional error. Due to the tight isolation cut, the measurement is sensitive to the fragmentation, i.e., the angular separation of the photons from charged particles.

The experiments agree in their findings that the models nicely describe the momentum dependence of the particle production. For the $\pi^{0}$, the differences are small and within the errors of the measurements; the $\eta$ measurements almost coincide, when extrapolated to the full momentum range. However, the $\eta^{\prime}$ rate observed by L3 exceeds the rate seen 

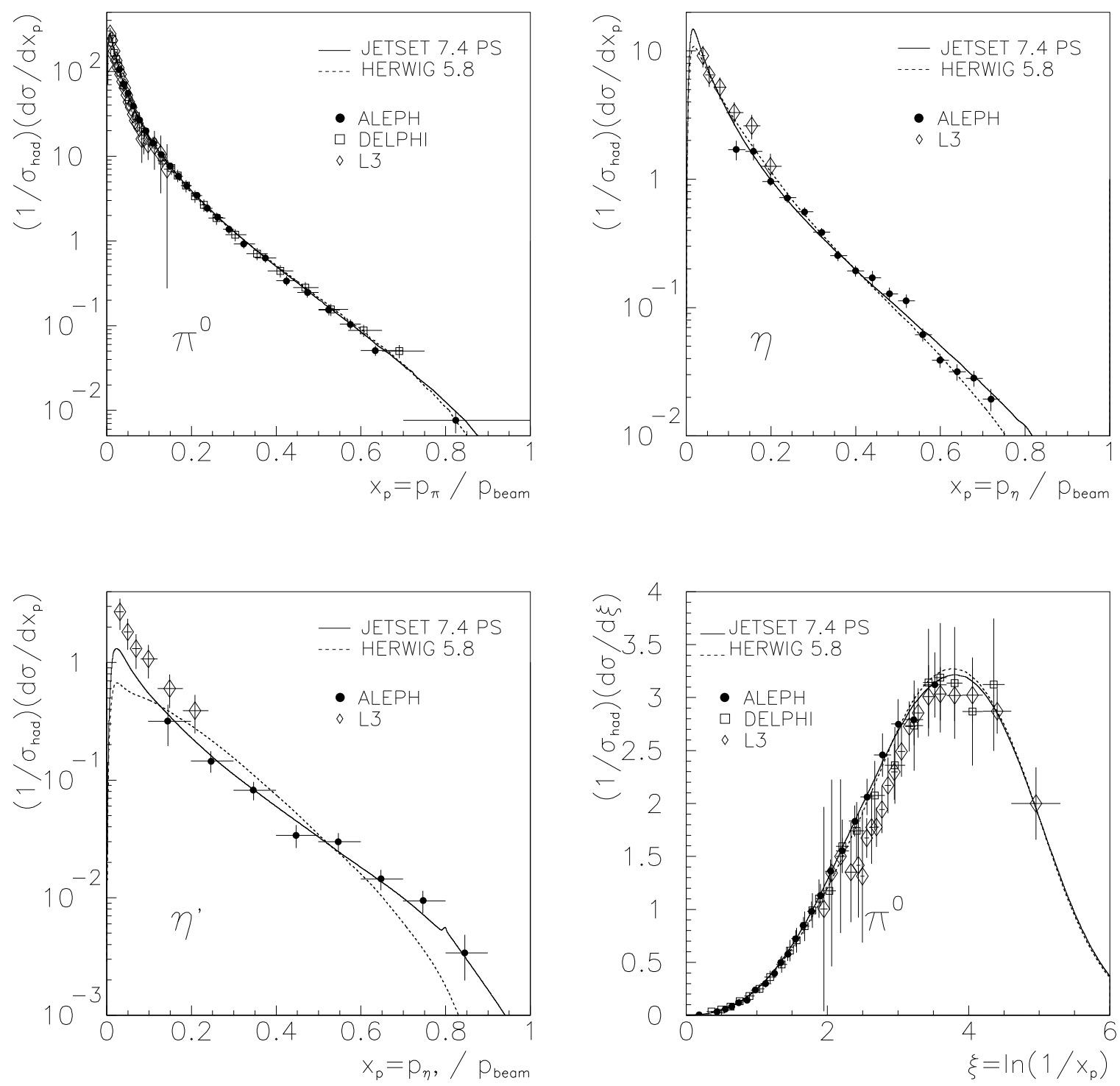

Figure 6.9: Momentum spectra for $\pi^{0}, \eta$, and $\eta^{\prime}$.

by ALEPH. These differences are significant even though the experiments provide spectra in different momentum ranges with small overlap only. Both results are a factor two or four lower than the standard JETSET model prediction. Therefore, an additional free parameter for $\eta^{\prime}$ suppression is introduced for describing the data in the model. It may be argued that the problem of the $\eta^{\prime}$ rate is an incorrect $\eta-\eta^{\prime}$ mixing angle $[79,110]$ in the theory or the Monte Carlo model. Studies show, however, that a change in the mixing angle alone, does not lead to a satisfactory solution [97]. The situation is complicated, because $64.5 \%$ of the $\eta^{\prime}$ contain an $\eta$ in their decay. The reduction of $\eta^{\prime}$ mesons lead to spectra, which now agree with data in shape, while the default JETSET tuning predicted 
a softer $\eta$ spectrum, i.e., lack of direct $\eta$ mesons. The different production probability of $\eta$ mesons in quark and gluon jets (see Section 7.5 ) is not properly reproduced by the models.

The understanding of the $\eta^{\prime}$ production is important for the understanding of the Bose-Einstein correlation. The pions are produced with small momentum difference $Q=$ $\sqrt{M^{2}-4 m_{\pi}^{2}}$, and alter the shape for pion pairs in a region, where the Bose-Einstein effect is studied. 


\subsection{Vector Mesons}

\section{Extraction of $\rho^{0}, \mathbf{K}^{* 0}, \mathbf{K}^{* \pm}, \omega$, and $\phi$ Signals}

\begin{tabular}{|c|c|c|c|c|c|}
\hline \multicolumn{2}{|c|}{ Particle } & $\begin{array}{c}\text { Mass } \\
{\left[\mathrm{MeV} / \mathrm{c}^{2}\right]}\end{array}$ & $\begin{array}{c}\text { Width } \\
{\left[\mathrm{MeV} / \mathrm{c}^{2}\right]}\end{array}$ & Decay mode & $\begin{array}{l}\mathrm{BR} \\
{[\%]}\end{array}$ \\
\hline 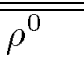 & $\overline{\mathrm{ADD}}$ & 769.9 & 151.2 & $\bar{~} \pi^{ \pm} \pi^{\mp}$ & 100.0 \\
\hline$K^{* \pm}$ & $\overline{\mathrm{ADO}}$ & 891.6 & 49.8 & $\begin{array}{l}\mathrm{K}_{\mathrm{s}}^{0} \pi^{\mp} \\
\hookrightarrow \pi^{ \pm} \pi^{\mp}\end{array}$ & $\begin{array}{l}33.3 \\
68.6\end{array}$ \\
\hline $\mathrm{K}^{* 0}$ & $\mathrm{ADO}$ & 896.1 & 50.5 & $\mathrm{~K}^{ \pm} \pi^{\mp}$ & 66.5 \\
\hline$\omega$ & $\begin{array}{r}\mathrm{AL} \\
\mathrm{L}\end{array}$ & 781.9 & 8.4 & $\begin{array}{l}\pi^{ \pm} \pi^{\mp} \pi^{0} \\
\quad \hookrightarrow \gamma \gamma \\
\pi^{0} \gamma \\
\hookrightarrow \gamma \gamma\end{array}$ & $\begin{array}{r}88.8 \\
98.8 \\
8.5 \\
98.8\end{array}$ \\
\hline$\phi$ & $\mathrm{ADO}$ & 1019.4 & 4.4 & $\mathrm{~K}^{ \pm} \mathrm{K}^{\mp}$ & 49.1 \\
\hline & & "Multiplici & /Event & & Ref. \\
\hline$\overline{\bar{\rho} \rho^{0}}$ & $\begin{array}{c}\text { ALEPH } \\
\text { DELPHI }\end{array}$ & $\begin{array}{l}1.45 \pm 0 . \\
1.21 \pm 0 .\end{array}$ & $\begin{array}{l}65 \pm 0.201 \\
4 \pm 0.14\end{array}$ & \pm 0.05 & $\begin{array}{l}{[23]} \\
{[19]}\end{array}$ \\
\hline $\mathrm{K}^{* \pm}$ & $\begin{array}{c}\text { ALEPH } \\
\text { DELPHI } \\
\text { OPAL }\end{array}$ & $\begin{array}{l}0.71 \pm 0 . \\
0.712 \pm 0 . \\
0.72 \pm 0 .\end{array}$ & $\begin{array}{l}6 \\
31 \pm 0.031 \\
2 \pm 0.08\end{array}$ & \pm 0.050 & $\begin{array}{l}{[24]} \\
{[19]} \\
{[25]}\end{array}$ \\
\hline $\mathrm{K}^{* 0}$ & $\begin{array}{c}\text { ALEPH } \\
\text { DELPHI } \\
\text { OPAL }\end{array}$ & $\begin{array}{l}0.830 \pm 0 . \\
0.97 \pm 0 . \\
0.743 \pm 0 .\end{array}$ & $\begin{array}{l}15 \pm 0.088 \\
8 \pm 0.31 \\
27 \pm 0.027\end{array}$ & & $\begin{array}{l}{[23]} \\
{[26]} \\
{[27]}\end{array}$ \\
\hline$\omega$ & $\begin{array}{r}\text { ALEPH } \\
\text { L3 }\end{array}$ & $\begin{array}{l}1.066 \pm 0 . \\
1.17 \pm 0 . \\
1.12 \pm 0 . \\
1.16 \pm 0 .\end{array}$ & $\begin{array}{l}58 \pm 0.124 \\
9 \pm 0.15 \\
2 \pm 0.25 \\
5 \text { (average) }\end{array}$ & $\begin{array}{l} \pm 0.044 \\
\left.\pi^{ \pm} \pi^{\mp} \pi^{0}\right) \\
\left.\pi^{0} \gamma\right)\end{array}$ & $\begin{array}{l}{[23]} \\
{[22]}\end{array}$ \\
\hline$\phi$ & $\begin{array}{r}\text { ALEPH } \\
\text { DELPHI } \\
\text { OPAL }\end{array}$ & $\begin{array}{l}0.122 \pm 0 . \\
0.090 \pm 0 . \\
0.100 \pm 0 .\end{array}$ & $\begin{array}{l}04 \pm 0.008 \\
08 \pm 0.011 \\
04 \pm 0.007\end{array}$ & & $\begin{array}{l}{[23]} \\
{[28]} \\
{[27]}\end{array}$ \\
\hline
\end{tabular}

Table 6.4: Properties of vector mesons and their measured average multiplicities.

Vector mesons decay strongly with their daughters originating from the primary interaction point, resulting in a large natural width. The combinatorial background may be large, but this can be reduced by; rejection of tracks from secondary vertices, tight cuts on the closest distance of the track to the primary interaction point and particle identification of their daughters.

The cross sections of the vector mesons are extracted from the invariant mass distributions of their daughters $\left(\pi^{ \pm}, \mathrm{K}^{ \pm}, \mathrm{K}_{\mathrm{s}}, \pi^{0}, \gamma\right)$. The distributions are fitted to a sum of a background and a signal function. 
The background can in general be represented by a smooth function. The specific choice is somewhat arbitrary and depends on the shape and amount of the combinatorial background. When the background is large, as for the $\rho^{0}$, it may be appropriate to subtract the two-particle mass spectrum of like-sign pions $m\left(\pi^{ \pm}, \pi^{ \pm}\right)$from the spectrum of the unlike-sign pions $m\left(\pi^{+}, \pi^{-}\right)$reducing systematics common to both two-pion spectra. This also makes the combinatorial background easier to parameterize. Some analyses also used the background shape from the Monte Carlo simulation for estimating the background in the data.

Some fits need the inclusion of reflections, e.g., in the $\mathrm{K}^{* 0}$ analysis: charged pions from the $\pi^{+} \pi^{-} \pi^{0}$ decay of the $\omega$ will give a structure in the $m\left(\mathrm{~K}^{ \pm} \pi^{\mp}\right)$ spectrum and must be taken into account as a separate contribution (Figure 4.2).

The signal is a convolution of a Breit-Wigner function, which describes the shape of the meson produced, and a resolution function, which accounts for the uncertainty in the momentum measurement in the detector. The Breit-Wigner function [101] used is

$$
B W(m)=\frac{m \cdot m_{0} \cdot \Gamma(m)}{\left(m^{2}-m_{0}^{2}\right)^{2}+m_{0}^{2} \cdot \Gamma^{2}(m)},
$$

with

$$
\Gamma(m)=\Gamma_{0} \cdot\left(\frac{q}{q_{0}}\right)^{2 l+1} \frac{m_{0}}{m} .
$$

Here, $m$ is the invariant mass of the particle pair, $m_{0}$ and $\Gamma_{0}$ are the mass and width of the studied vector meson. One has $l=1$ for vector mesons; $q$ is the momentum of the decay products in the rest frame of the parent, and $q_{0}$ is the momentum when $m=m_{0}$. The choices made by the experiments for the width function $\Gamma(m)$, which is a function of mass, are slightly different. Other parameterizations can be found in Ref. [101].

The way the resolution is taken into account depends on the natural width of the meson relative to the detector resolution. Thus the treatment differs in detail for the mesons under study. For the $\mathrm{K}^{* 0}$ and $\mathrm{K}^{* \pm}$, the uncertainty from the resolution can be neglected. For the $\phi$, a convolution is made which depends on momentum: DELPHI uses a Gaussian for the resolution, ALEPH a non-relativistic Breit-Wigner function, OPAL the shape from the Monte Carlo. For the $\omega$, the energy resolution of the calorimeter dominates the shape of the signal and a Gaussian (L3: free width and mass for $\omega \rightarrow \pi^{0} \gamma$ and $\omega \rightarrow \pi^{+} \pi^{-} \pi^{0}$ ) or a sum of Gaussians (ALEPH: fixed width and mass for $\omega \rightarrow \pi^{+} \pi^{-} \pi^{0}$ ) is used.

For the $\rho^{0}$, which has a large width $\left(151.5 \pm 1.2 \mathrm{MeV} / c^{2}\right)$, the detector resolution is not important. However, Bose-Einstein correlations (see Section 7.7) may distort the $\rho^{0}$ line shape through interactions between the $\rho^{0}$ decay pions and other pions. Therefore different strategies had to be invoked. OPAL finds with JETSET Monte Carlo studies that the inclusion of Bose-Einstein correlations improves the description of the invariant mass spectrum of pions in data. A good agreement can only be obtained for a high value of the chaoticity parameter $\lambda=2.5$; however, no $\rho^{0}$ multiplicity was extracted. DELPHI obtained a $\rho^{0}$ rate using a Breit-Wigner function for the signal and finds a large mass shift. In a second analysis they use JETSET with parameters for the chaoticity parameter and the inverse source size $1 / \sigma$ as measured experimentally: $\lambda=1$ and $1 / \sigma=0.4 \mathrm{GeV} / \hbar c$. 
ALEPH relies on the JETSET model as well, using JETSET modified to include the BreitWigner function described above for the $\rho^{0}$ and the $\mathrm{K}^{* 0}$, both signal and background shape were taken from the Monte Carlo. The finding of $\lambda \approx 2.1$ and $1 / \sigma=0.35 \mathrm{GeV} / \hbar c$ is compatible with OPAL's value in view of the different $\eta^{\prime}$ rate and choice of the coherence time parameter $\chi\left(0.1 \mathrm{GeV} / c^{2}\right.$ for ALEPH, while OPAL and DELPHI use $\left.0.02 \mathrm{GeV} / c^{2}\right)$. This parameter gives the minimum width of resonances whose daughters contribute to the Bose-Einstein enhancement (see Section 7.7 for a more general discussion of the BoseEinstein correlations).

\section{Momentum Spectra}

Despite the large natural widths of the studied vector mesons and the amount of combinatorial background, we see in Figures 6.10 a surprisingly good agreement among the various measurements. Both shapes and rates are reproduced by both Monte Carlo models.

For the $\rho^{0}$ line shape, however, the mass is shifted to lower values. While for high $\rho^{0}$ momenta the shift of the peak position is negligible, the mass shift at lower momenta is 10 to $20 \mathrm{MeV} / \mathrm{c}^{2}$, an effect which has not be seen at lower centre-of-mass energies. Particle multiplicity may play a role. In the framework of the JETSET model the shift or change of line shape can be interpreted as an effect of Bose-Einstein correlations (see Section 7.7). Interference with the background or with the $\omega$ is not excluded as alternative explanation. Some problems arise from the $\mathrm{K}^{* 0}$ reflection, which is close in the invariant mass spectrum.

Small differences are seen for the $\phi$ spectrum: while the measurements coincide at high momentum the measured spectra tend to diverge for lower momenta. The models predict a harder spectrum than all three measurements. A similar trend, though weaker, is seen for the $\mathrm{K}^{*}$ mesons. For all vector mesons, but most prominent for the $\mathrm{K}^{*}$ mesons, the inclusion in the analysis of the production of mesons with orbital angular momentum $\mathrm{L}=1$ was found to be important, especially at low momenta. It both reduced the expected rate and softened the momentum spectra at low $x_{p}$. 


\subsection{Scalar and Tensor Mesons}

\section{Extraction of $\mathbf{f}_{0}, \mathbf{f}_{2}, \mathbf{f}_{2}^{\prime}$, and $\mathbf{K}_{2}^{* 0}$ Signals}

\begin{tabular}{|c|c|c|c|c|c|}
\hline & $\begin{array}{l}\text { article } \\
\text { Exp. }\end{array}$ & $\begin{array}{c}\text { Mass } \\
{\left[\mathrm{MeV} / \mathrm{c}^{2}\right]}\end{array}$ & $\begin{array}{c}\text { Width } \\
{\left[\mathrm{MeV} / \mathrm{c}^{2}\right]}\end{array}$ & Decay mode & $\begin{array}{l}\mathrm{BR} \\
{[\%]}\end{array}$ \\
\hline$\overline{f_{0}}$ & $\overline{\mathrm{D}}$ & 980 & $40-400$ & $\overline{\pi^{ \pm} \pi^{\mp}}$ & 52.1 \\
\hline$f_{2}$ & $\mathrm{D}$ & 1275 & 185 & $\pi^{ \pm} \pi^{\mp}$ & 56.6 \\
\hline$f_{2}^{\prime}$ & $\mathrm{D}$ & 1525 & 76 & $\mathrm{~K}^{ \pm} \mathrm{K}^{\mp}$ & 35.6 \\
\hline $\mathrm{K}_{2}^{* 0}$ & $\mathrm{O}$ & 1432 & 109 & $\mathrm{~K}^{ \pm} \pi^{\mp}$ & 33.1 \\
\hline & & \multicolumn{3}{|c|}{ Multiplicity/Event } & Ref. \\
\hline 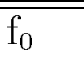 & DELPHI & \multicolumn{3}{|c|}{$0.098 \pm 0.016\left(0.05<x_{E}<0.6\right)$} & $\overline{[19]}$ \\
\hline$f_{2}$ & DELPHI & \multicolumn{3}{|c|}{$0.170 \pm 0.043\left(x_{E}>0.05\right)$} & $19]$ \\
\hline & DELPHI & \multicolumn{3}{|c|}{$0.020 \pm 0.005 \pm 0.006$} & $29]$ \\
\hline $\mathrm{K}_{2}^{* 0}$ & OPAL & \multicolumn{3}{|c|}{$0.19 \pm 0.04 \pm 0.06\left(x_{E}<0.3\right)$} & $27]$ \\
\hline
\end{tabular}

Table 6.5: Properties of scalar and tensor mesons and their measured average multiplicities.

The extraction of the number of scalar and tensor mesons with a large natural width proceeds in the same manner as for the vector mesons, with a fit to the two-particle invariant mass spectrum.

The signal shape is parameterized by Breit-Wigner functions (see Section 6.4) with $l=0$ and $l=2$. The distortion due to Bose-Einstein correlations has been taken into account $(\lambda=1)$ for the $f_{2}$, but not for the $f_{0}$.

In the $\mathrm{f}_{2}^{\prime}$ analysis, the fit to the $\mathrm{K}^{+} \mathrm{K}^{-}$mass spectrum is improved at low momenta when the $f_{J}(1710)$ is included. However, the production rate for the $f_{J}(1710)$ is low, $405 \pm 200$ events are seen.

For the $\mathrm{K}_{2}^{* 0}$ analysis special care was necessary for describing the background, where the shape was controlled using side-bands. The particles could only be measured over a restricted momentum range.

\section{Momentum Spectra}

The predicted slope of the spectra of $\mathrm{f}_{0}, \mathrm{f}_{2}$, and $\mathrm{f}_{2}^{\prime}$ in Figure 6.11 is in agreement with the data. The predicted production rate of the $\mathrm{f}_{0}$, only available for JETSET, is much too low. This may be due to the tuning, which is a compromise for describing various spectra but with little input from scalar and tensor mesons. One may as well speculate, whether the $\mathrm{f}_{0}$ is not a $\mathrm{q} \overline{\mathrm{q}}$ state, but rather a $\mathrm{KK}$ molecule [97]?

The JETSET generator the standard parameters for the scalar and tensor production probability can be replaced: the DELPHI collaboration has introduced new parameters, which determine the production probability of mesons with different orbital angular momentum for all quark flavours independently. The studies suggest that the excited mesons 

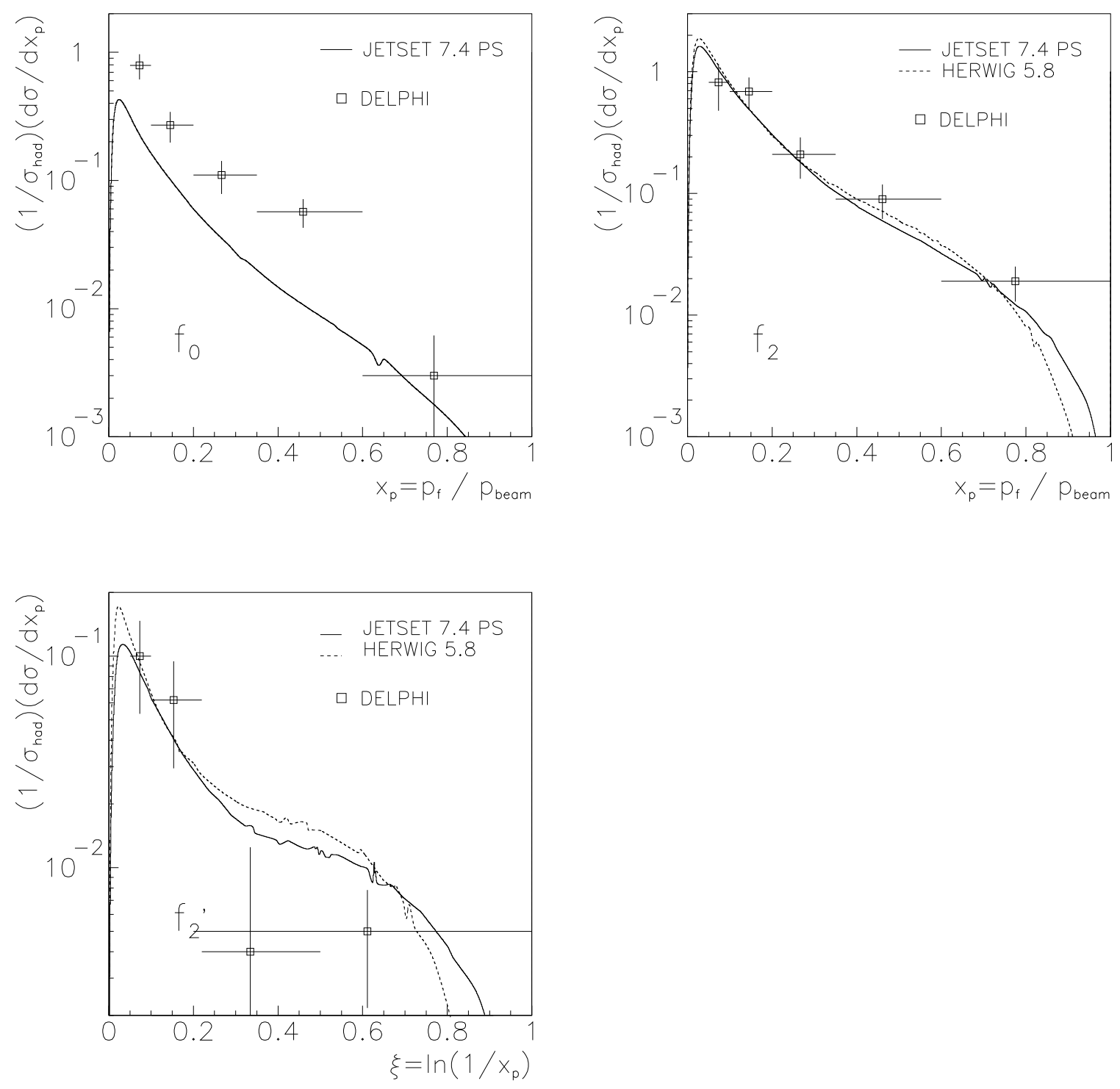

Figure 6.11: Momentum spectra for $f_{0}, f_{2}$, and $f_{2}^{\prime}$.

have a production probability of about $40 \%$ [99]. This is much higher than expected from early estimations of about $10 \%$ using the string model [111], based on string length, hadron size and average $p_{t}$. More reliable estimations take into account that a string piece with large $p_{t}$ is shorter and that the contribution to the angular momentum from the quark and anti-quark are not parallel [112]; in conclusion, the measured $40 \%$ may be not unnaturally large.

The DELPHI collaboration has compared their tensor productions with the vector meson production of $\rho^{0}$ and $\phi$. They find ratios $\sigma\left(\mathrm{f}_{2}^{\prime}\right) / \sigma(\phi)=0.19 \pm 0.07$ and $\sigma\left(\mathrm{f}_{2}\right) / \sigma\left(\rho^{0}\right)=$ $0.24 \pm 0.07$, in nice agreement with one another, showing that the $f_{2}^{\prime}$ is an $s \bar{s}$ tensor. The 
comparison of the shapes of the momentum spectra, $f_{2}$ versus $\rho^{0}$ and $f_{2}^{\prime}$ versus $\phi$ confirm this. Though the $f_{0}$ rate is not reproduced by our present tuning (see Section 3.4 ), the DELPHI collaboration states that the $\mathrm{f}_{0} / \rho^{0}$ ratio as function of $x_{p}$ is flat, which implies a similar production mechanism for these mesons.

More precise measurements of these and other particles with orbital angular momentum are needed; the influence on the vector meson production has been pointed out in the previous section. For example, $\mathrm{a}_{0}, \mathrm{a}_{1}, \mathrm{~b}_{1}, \mathrm{f}_{1}$, and $\mathrm{h}_{1}$ should have production probabilities similar to $f_{0}$ as suggested by JETSET; the $a_{2}$ rates should be of the order of the $f_{2}$ rate. Knowledge of at least some of these particles would help to understand the production of particles with orbital angular momentum. 


\subsection{Non-Strange Baryons}

\section{Extraction of the $\Delta^{++}$Signal}

\begin{tabular}{|c|c|c|c|c|c|}
\hline \multicolumn{2}{|c|}{$\begin{array}{l}\text { Particle } \\
\text { Exp. }\end{array}$} & $\begin{array}{c}\text { Mass } \\
{\left[\mathrm{MeV} / \mathrm{c}^{2}\right]}\end{array}$ & $\begin{array}{c}\text { Width } \\
{\left[\mathrm{MeV} / \mathrm{c}^{2}\right]}\end{array}$ & Decay mode & $\begin{array}{l}\mathrm{BR} \\
{[\%]}\end{array}$ \\
\hline \multirow[t]{2}{*}{$\Delta^{++}$} & $\mathrm{DO}$ & 1232 & 120 & $\mathrm{p} \pi^{+}$ & 100.0 \\
\hline & & \multicolumn{3}{|c|}{ Multiplicity/Event } & Ref. \\
\hline$\overline{\Delta^{++}}$ & $\begin{array}{r}\text { DELPHI } \\
\text { OPAL }\end{array}$ & \multicolumn{3}{|c|}{$\begin{array}{l}0.079 \pm 0.009 \pm 0.009 \pm 0.007 \\
0.22 \pm 0.04 \pm 0.04\end{array}$} & $\begin{array}{l}30] \\
{[31]}\end{array}$ \\
\hline
\end{tabular}

Table 6.6: Properties of non-strange baryons and their measured average multiplicities.

The four particles with the lowest mass in the light-baryon decuplet are the $\Delta$ baryons. The resonances have a width $\Gamma \approx 120 \mathrm{MeV} / c^{2}$ and the signal extraction has to cope with a very large combinatorial background. Only the production rate of the $\Delta^{++}\left(\Delta^{++} \rightarrow \mathrm{p} \pi^{+}\right)$ has been measured before at lower centre-of-mass energies [113]. A reliable signal can only be obtained with a good particle identification, especially for the protons. The specific ionization loss $\mathrm{d} E / \mathrm{d} x$ has its best separation power at low momenta for protons. However, the kinematics of the decay favours $p_{\mathrm{p}}>p_{\pi}$, a condition explicitly required by the analysis of the OPAL collaboration, worsening the situation. In addition, the number of charged pions is highest at low momentum. This leads to the fact that the position of the $\Delta^{++}$ signal coincides with the maximum of the combinatorial background from phase space. Therefore, the two experiments, having measured the $\Delta^{++}$production rate, use a TOF system (OPAL) or the RICH detector (DELPHI) as an additional particle identification tool.

The fit to the $\mathrm{p} \pi^{+}$spectrum uses a sum of a relativistic Breit-Wigner function as signal shape and an analytic function for the background. For the signal, the exact knowledge of the resolution is not important. DELPHI reports a distortion of the $\Delta^{++}$ line shape; however, the influence on the extracted number of $\Delta^{++}$'s is found to be small $(\approx 5 \%)$, when the effect is studied with the Bose-Einstein correlations as implemented in the JETSET model. OPAL made a detailed study on the background shape; sixteen background shapes were obtained from Monte Carlo, which consisted of all possible twoparticle combinations of $\mathrm{p}, \pi^{+}, \mathrm{K}^{+}$, and all other charged tracks.

\section{Momentum Spectra}

The measurement of the $\Delta^{++}$is certainly a very difficult one and the errors are large. Nevertheless, there is a factor of three between the two measurements, which is significant. The other isospin states of the $\Delta$ baryon, if measurable, may give further insight, but the combinatorial background will be even larger. Information from other particles does not (yet) lead to a conclusion, on which measurement is right or wrong. The tuned Monte 


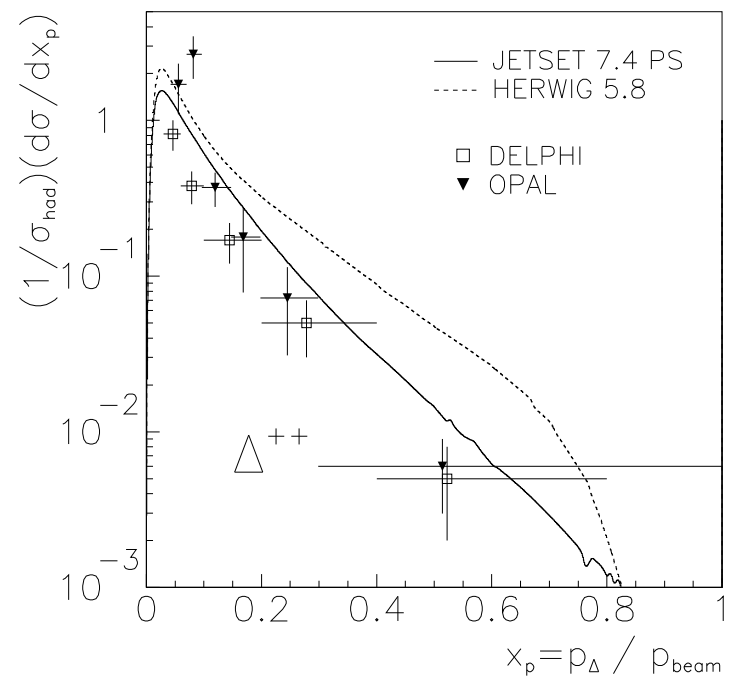

Figure 6.12: Momentum spectra for $\Delta^{++}$.

Carlo models prefers the OPAL multiplicity, but the shape from the DELPHI experiment. The model of Chliapnikov [82] favours the DELPHI number (see Section 7.1).

Measurements from other experiments should help to solve this discrepancy. Studies should consider the influence of the protons from $\Delta$ decays and their impact on the proton momentum spectrum. When the production of the other isospin states is taken into account more protons originate from $\Delta$ baryons than from $\Lambda$; a good $\Delta^{++}$measurement may give an answer to the problem of a simultaneous tuning of the proton and $\Lambda$ spectra. 


\subsection{Strange Baryons}

Extraction of the $\Sigma^{-}, \Sigma^{0}, \Sigma^{+}, \Xi^{-}, \Sigma^{* \pm}, \Xi^{* 0}$, and $\Omega^{-}$Signals

\begin{tabular}{|c|c|c|c|c|c|}
\hline \multicolumn{2}{|c|}{ Particle } & $\begin{array}{c}\text { Mass } \\
{\left[\mathrm{MeV} / \mathrm{c}^{2}\right]}\end{array}$ & $\begin{array}{c}\text { Width }(c \tau) \\
{\left[\mathrm{MeV} / \mathrm{c}^{2}(\mathrm{~cm})\right]}\end{array}$ & Decay mode & $\begin{array}{l}\mathrm{BR} \\
{[\%]}\end{array}$ \\
\hline \multirow{2}{*}{$\overline{\Sigma^{+}}$} & $\overline{\overline{\mathrm{D}}}$ & 1189.4 & $(2.4)$ & $\overline{\mathrm{nn} \pi^{+}}$ & $\overline{48.3}$ \\
\hline & $\mathrm{O}$ & 1189.4 & $(2.4)$ & $\mathrm{p} \pi^{0}$ & $\begin{array}{l}51.6 \\
98.8\end{array}$ \\
\hline$\Sigma^{-}$ & $\mathrm{DO}$ & 1189.4 & $(4.4)$ & $\mathrm{n} \pi^{-}$ & 99.8 \\
\hline$\Sigma^{0}$ & DO & 1192.6 & $\left(2 \cdot 10^{-9}\right)$ & $\stackrel{\Lambda \gamma}{\hookrightarrow p \pi^{-}}$ & $\begin{array}{r}100.0 \\
63.9\end{array}$ \\
\hline$\Xi^{-}$ & ADO & 1321 & $(4.9)$ & $\begin{array}{l}\Lambda \pi^{-} \\
\hookrightarrow \mathrm{p} \pi^{-}\end{array}$ & $\begin{array}{l}99.9 \\
63.9\end{array}$ \\
\hline$\Sigma^{* \pm}$ & ADO & 1385 & 37.6 & $\begin{array}{l}\Lambda \pi^{ \pm} \\
\hookrightarrow \mathrm{p} \pi^{-}\end{array}$ & $\begin{array}{l}88 . \\
63.9\end{array}$ \\
\hline$\Xi^{* 0}$ & $\mathrm{ADO}$ & 1532 & 9.1 & $\begin{array}{l}\Xi^{-} \pi^{+} \\
\hookrightarrow \Lambda \pi^{-} \\
\quad \hookrightarrow \mathrm{p} \pi^{-}\end{array}$ & $\begin{array}{l}66.7 \\
99.9 \\
63.9\end{array}$ \\
\hline \multirow[t]{2}{*}{$\overline{\Omega^{-}}$} & ADO & 1672 & $(2.5)$ & $\begin{array}{l}\Lambda \mathrm{K}^{-} \\
\hookrightarrow \mathrm{p} \pi^{-}\end{array}$ & $\begin{array}{l}67.8 \\
63.9 \\
\end{array}$ \\
\hline & & \multicolumn{3}{|c|}{ Multiplicity/Event } & $\overline{\text { Ref. }}$ \\
\hline$\overline{\Sigma^{ \pm}}$ & DELPHI & \multicolumn{3}{|c|}{$0.170 \pm 0.014 \pm 0.061\left(\Sigma^{+}+\Sigma^{-}\right)$} & $\overline{[\overline{[36]}}$ \\
\hline$\Sigma^{+}$ & OPAL & \multicolumn{3}{|c|}{$0.092 \pm 0.008 \pm 0.009 \pm 0.012$} & {$[35]$} \\
\hline$\Sigma^{-}$ & OPAL & \multicolumn{3}{|c|}{$0.084 \pm 0.009 \pm 0.008 \pm 0.013$} & [35] \\
\hline \multirow[t]{2}{*}{$\Sigma^{0}$} & DELPHI & \multicolumn{3}{|c|}{$0.070 \pm 0.010 \pm 0.010$} & [34] \\
\hline & OPAL & \multicolumn{3}{|c|}{$0.078 \pm 0.014 \pm 0.013 \pm 0.014$} & [35] \\
\hline \multirow[t]{3}{*}{$\Xi^{-}$} & ALEPH & \multicolumn{3}{|c|}{$0.0297 \pm 0.00057 \pm 0.0020$} & {$[37]$} \\
\hline & DELPHI & \multicolumn{3}{|c|}{$0.0250 \pm 0.0009 \pm 0.0021$} & [36] \\
\hline & OPAL & \multicolumn{3}{|c|}{$0.0240 \pm 0.0007 \pm 0.0017$} & {$[33]$} \\
\hline \multirow[t]{3}{*}{$\Sigma^{* \pm}$} & ALEPH & \multicolumn{3}{|c|}{$0.065 \pm 0.004 \pm 0.008$} & [37] \\
\hline & DELPHI & \multicolumn{3}{|c|}{$0.0382 \pm 0.0028 \pm 0.0045$} & {$[36]$} \\
\hline & OPAL & \multicolumn{3}{|c|}{$0.0372 \pm 0.0032 \pm 0.0039$} & [33] \\
\hline \multirow[t]{3}{*}{$\Xi^{* 0}$} & ALEPH & \multicolumn{3}{|c|}{$0.0072 \pm 0.0004 \pm 0.0006$} & [37] \\
\hline & DELPHI & \multicolumn{3}{|c|}{$0.0041 \pm 0.0004 \pm 0.004$} & {$[36]$} \\
\hline & OPAL & \multicolumn{3}{|c|}{$0.0072 \pm 0.0010 \pm 0.0009$} & {$[33]$} \\
\hline \multirow[t]{3}{*}{$\Omega^{-}$} & ALEPH & \multicolumn{3}{|c|}{$0.0010 \pm 0.0002 \pm 0.0001$} & {$[37]$} \\
\hline & DELPHI & \multirow{2}{*}{\multicolumn{3}{|c|}{$\begin{array}{l}0.0014 \pm 0.0002 \pm 0.0004 \\
0.0028 \pm 0.0008 \pm 0.0003\end{array}$}} & {$[34]$} \\
\hline & OPAL & & & & [33] \\
\hline
\end{tabular}

Table 6.7: Properties of strange baryons and their measured average multiplicities. 
For the extraction of the charged $\Sigma$ hyperons, DELPHI and OPAL use different strategies. The $\Sigma^{+}$, with $c \tau=2.4 \mathrm{~cm}$, is measured in its decay mode $\Sigma^{+} \rightarrow \mathrm{p} \pi^{0}(B R=51.6 \%)$ by OPAL. The impact parameter of the proton track in the plane transverse to the beam line should be inconsistent with zero $(5 \sigma)$, but lower than $2 \mathrm{~cm}$ in order to decrease combinatorial background. Charged pions and kaons are rejected by their energy loss $\mathrm{d} E / \mathrm{d} x$; protons are rejected if they are daughters of $\Lambda$ candidates. The closest approach of the proton to the beam line is taken as the $\Sigma^{+}$decay point. Combined with a neutral pion, the total momentum of the $\Sigma^{+}$is calculated. Additional cuts ( $z$ impact, decay probability) are applied; the $\mathrm{p} \pi^{0}$ spectrum is fitted as a sum of a Gaussian and an analytic background function. The $\Sigma^{-}$is extracted measuring only the $\pi^{-}$as decay product: a track with a kink, i.e., a primary track, which ends inside the tracking chamber, where a secondary track starts, is searched for. Only one secondary track is allowed, which must have the same charge as the primary one. The momentum of the neutron is calculated from the primary $\left(\Sigma^{-}\right)$and secondary $\left(\pi^{-}\right)$track. Assigning the neutron mass, the invariant mass from the neutron and $\pi^{-}$is plotted. The background from decay-in-flight (esp. $\mathrm{K}^{ \pm}$), however, is high. Therefore, a simultaneous fit to the invariant mass, the proper time, and $\cos \theta^{*}$ of the pion in the $\Sigma^{-}$rest frame is performed. The rates of the background from $\mathrm{K}^{ \pm}$and $\Xi^{-}$, which agree with rates from other analyses, are taken as a check. DELPHI uses the decay mode $n \pi^{ \pm}$for both charged baryons. Similar to the $\Sigma^{-}$analysis in OPAL a kink is searched for; from the calculated neutron direction, the shower in the hadron calorimeter is found. Then the $n \pi^{ \pm}$mass spectrum is evaluated. The method is checked with wrong charge combinations.

The measurement of the other hyperons relies on the tracking devices of the detectors and all final state particles are measured. All five hyperons are tagged by their decay via a $\Lambda$. The $\Lambda$ is selected as described above and in most of the analyses no kinematical fit is made and no cut is applied on the impact parameter of the $\Lambda$. Only a window of typically $\pm 2 \sigma$ around the expected $\Lambda$ mass is used.

The clear signature of the $\Sigma^{0}$ is used for its detection: a $\Lambda$ is combined with a low energetic photon. The mass difference between $\Sigma^{0}$ and $\Lambda\left(\Delta m=1192.55 \mathrm{MeV} / c^{2}-\right.$ $\left.1115.63 \mathrm{MeV} / c^{2}=76.92 \mathrm{MeV} / c^{2}\right)$ is too small to allow a strong decay; hence the $\Sigma^{0}$ appears as a narrow peak. To exploit this, photons converting into an $\mathrm{e}^{+} \mathrm{e}^{-}$-pair in the detector are used. The good momentum resolution of the tracking devices results in a momentum measurement better than the calorimetric one. The efficiency, however, is rather low (few percent). The $\Lambda$ is reconstructed as described above, with the constraint that the flight direction points back to the beam collision point. The $\Lambda \gamma$ mass spectrum is fitted with a Gaussian function for the signal and an analytic function for the background.

For the other four hyperons, the $\Lambda$ baryon is combined with charged tracks, which must agree with the expected energy loss $\mathrm{d} E / \mathrm{d} x$ of pion, kaon, or proton. The $\Xi^{-}$and $\Omega^{-}$, which have a long lifetime, are identified by a secondary vertex formed by the trajectories of the $\Lambda$ and $\pi^{-}$or $\mathrm{K}^{-}$. This additional charged track, which must not pass through the primary vertex, is combined with the $\Lambda$; the resulting particle momentum pointing back to the collision point. The distance of the two secondary vertices has to correspond (e.g., in ALEPH) between 0.2 and 5 proper lifetimes of the $\Lambda$. The signature being similar, with a smaller production rate of the $\Omega^{-}$, identified $\Xi^{-}$baryons are excluded as $\Omega^{-}$candidates. 
DELPHI has made a simultaneous fit of the two vertices for their $\Omega^{-}$extraction, while using the $\Xi^{-}$to check the efficiency, which critically depends on the understanding of the $\chi^{2}$ distributions of the fit.

The fit to the $\Lambda \pi^{-}$and $\Lambda \mathrm{K}^{-}$spectra is composed of a signal, assumed Gaussian, and an analytic function for the background shape, which was checked with the wrong-sign combination spectrum $\left(\Lambda \pi^{+}, \Lambda \mathrm{K}^{+}\right)$and using side bands.

The $\Xi^{* 0}$, which decays strongly, is formed combining $\Xi^{-}$candidates within a mass window with identified pions of the right $\operatorname{sign}\left(\pi^{+}\right)$, with small impact parameter. The signal is taken as a convolution of a Breit-Wigner function and a Gaussian. As an alternative, the number of $\Xi^{* 0}$ is taken as number of combinations above background. The background is taken as the shape of the $\Xi^{-} \pi^{-}$mass distribution. The normalization is left free, because correlations are different for the two different charge combinations, e.g., the production of $\Xi^{-}$and $\mathrm{K}^{+}$is correlated, because of local strangeness compensation. Another background estimation is obtained, when the wrong-sign combinations $\left(\Lambda \pi^{+}\right)$for the $\Xi^{-}$are taken.

The extraction of the wide $\Sigma^{* \pm}$ resonance proceeds similarly to the measurement of the vector mesons. The mass spectrum of $\Lambda$ 's and charged pions, with small impact parameter, is fitted as sum of a Breit-Wigner and a background function. The extraction is difficult, with the width of the $\Sigma^{* \pm}$ being large and in the presence of a $\Xi^{-}$reflection, which is close in mass. Therefore, several checks on the background have been performed: study of the wrong-sign combination, which removes the $\Xi^{-}$reflection; replacement of the background by the Monte Carlo shape; and taking fake $\Lambda$ baryons, i.e., the analysis is performed with $\mathrm{p}$ and $\pi^{-}$combinations not consistent with the $\Lambda$ mass.

\section{Momentum Spectra}

The OPAL collaboration shows that their $\Sigma^{+}$and $\Sigma^{-}$spectra, evaluated separately, have the same production rate and the same momentum spectrum, which is in agreement with the Monte Carlo model prediction. For the $\Sigma^{0}$ only the rate is measured, which is correctly simulated in the models.

The JETSET model describes all four of the measured momentum spectra. The HERWIG model describes he shapes reasonably for low momenta; in fact, all strange hyperon momentum spectra are too hard in HERWIG and the production rates are overestimated. For this reason, the hyperons have actually been excluded in the tuning. The JETSET model, with its more parameters, can describe the data both for the production rate and the momentum dependence (Figure 6.13 ). Even the $\Omega^{-}$production is correctly predicted! This is surprising, because at PEP/PETRA energies, an exceptionally high rate was observed. JETSET is now the favoured model for the understanding of the $\Omega^{-}$production, where the ss-diquark is governed by the tunneling only (see remarks in Section 7.2).

More general studies such as the measurement of strangeness suppression may help (see Section 7.2) to cure the overestimation of strange baryon production in HERWIG. Implementation of special parameters into HERWIG, e.g., for strange diquark and leading baryon suppression, in the model tuning may lead to an improvement, as performed with sc Jetset. (In the newer versions of HERWIG parameters for suppression of some multiplets 

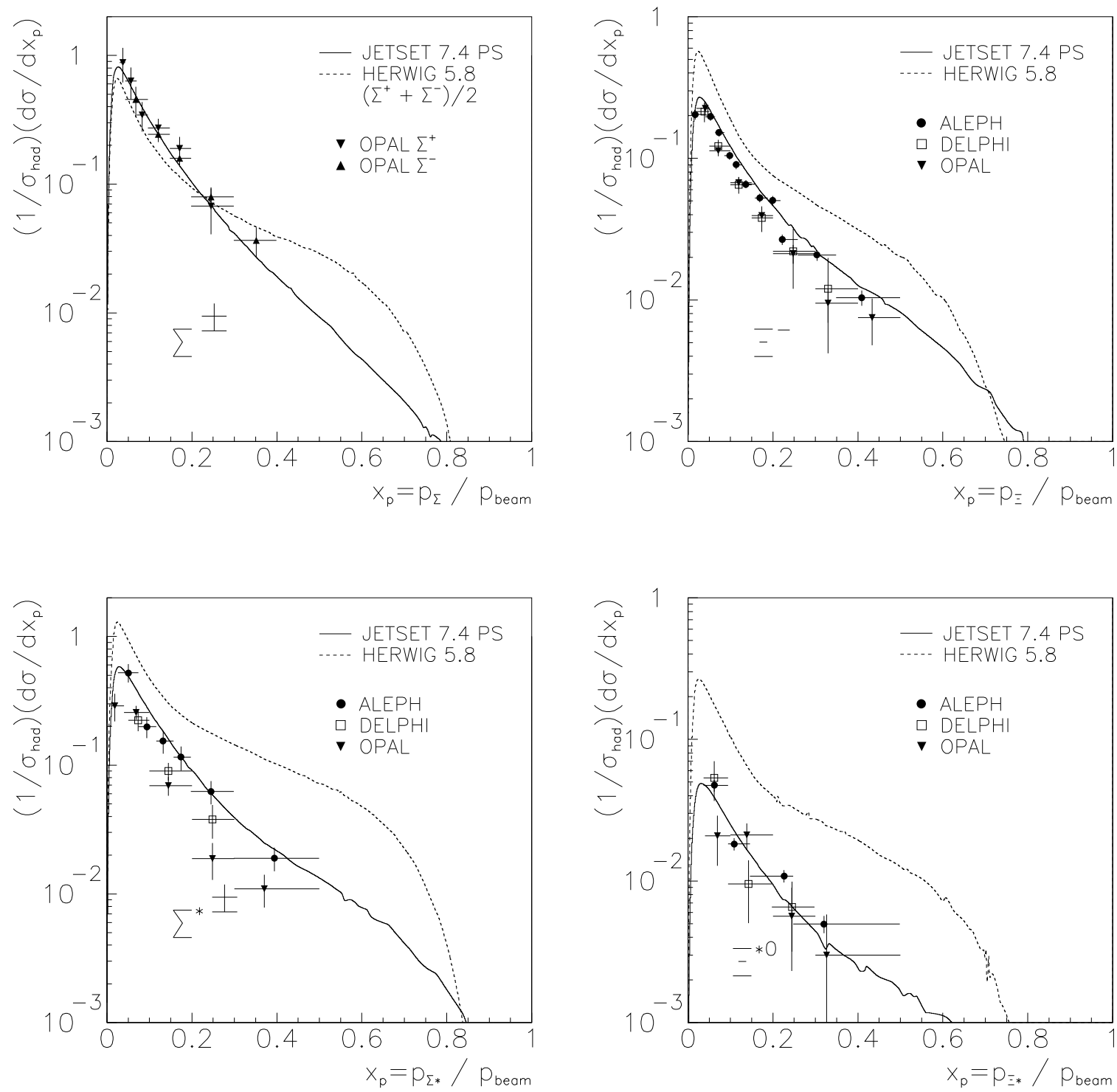

Figure 6.13: Momentum spectra for strange baryons.

(vector mesons, tensors, and decuplet-baryons) are introduced; the parameters have not yet been used for model tuning.) 


\subsection{Charmed Hadrons}

\section{Extraction of the $\mathbf{D}^{0}, \mathbf{D}^{ \pm}, \mathbf{D}^{* \pm}, \mathbf{D}^{* * 0}, \mathbf{D}_{\mathrm{s}}^{+}$, and $\Lambda_{\mathrm{c}}$ Signals}

\begin{tabular}{|c|c|c|c|c|c|}
\hline \multicolumn{2}{|c|}{ Particle } & $\begin{array}{c}\text { Mass } \\
{\left[\mathrm{MeV} / \mathrm{c}^{2}\right]}\end{array}$ & $\begin{array}{c}\text { Width }(c \tau) \\
{\left[\mathrm{MeV} / c^{2}(\mu \mathrm{m})\right]}\end{array}$ & Decay mode & $\begin{array}{l}\mathrm{BR} \\
{[\%]}\end{array}$ \\
\hline $\mathrm{D}^{0}$ & ADO & 1865 & (124) & $\mathrm{K}^{-} \pi^{+}$ & 4.01 \\
\hline $\mathrm{D}^{ \pm}$ & ADO & 1869 & $(317)$ & $\mathrm{K}^{\mp} \pi^{ \pm} \pi^{ \pm}$ & 9.1 \\
\hline $\mathrm{D}^{* \pm}$ & ADO & 2010 & $<0.131$ & $\begin{array}{l}\mathrm{D}^{0} \pi^{+} \\
\hookrightarrow \mathrm{K}^{-} \pi^{+}\end{array}$ & $\begin{array}{l}68.1 \\
4.01\end{array}$ \\
\hline $\mathrm{D}^{* * 0}$ & A & $2420 / 2460$ & $18 / 23$ & $\begin{array}{l}\mathrm{D}^{*+} \pi^{-} \\
\hookrightarrow \mathrm{D}^{0} \pi^{+} \\
\quad \hookrightarrow \mathrm{K}^{-} \pi^{+}\end{array}$ & $\begin{array}{l}\text { seen } \\
68.1 \\
4.01\end{array}$ \\
\hline $\mathrm{D}_{\mathrm{s}}^{+}$ & ADO & 1969 & 140 & $\begin{array}{l}\phi \pi^{+} \\
\overline{\mathrm{K}}^{* 0} \mathrm{~K}^{+}\end{array}$ & $\begin{array}{l}3.5 \\
3.3\end{array}$ \\
\hline$\Lambda_{c}^{+}$ & $\mathrm{O}$ & 2285 & $(60)$ & $\mathrm{pK}^{-} \pi^{+}$ & 4.3 \\
\hline & & Multiplicity & /Event & & Ref. \\
\hline & $\begin{array}{r}\text { ALEPH } \\
\text { DELPHI } \\
\text { OPAL }\end{array}$ & $\begin{array}{l}0.479 \pm 0.0 \\
0.454 \pm 0.0 \\
0.437 \pm 0.0\end{array}$ & $\begin{array}{l}8 \pm 0.018 \\
2 \pm 0.030 \pm 0.0 \\
5 \pm 0.021 \pm 0.0\end{array}$ & $\begin{array}{l}6^{\dagger}(\mathrm{BR}) \\
5^{\dagger}(\mathrm{BR})\end{array}$ & $\begin{array}{l}{[38]} \\
{[39]} \\
{[40]}\end{array}$ \\
\hline $\mathrm{D}^{ \pm}$ & $\begin{array}{r}\text { ALEPH } \\
\text { DELPHI } \\
\text { OPAL }\end{array}$ & $\begin{array}{l}0.221 \pm 0.0 \\
0.188 \pm 0.0 \\
0.165 \pm 0.0\end{array}$ & $\begin{array}{l}3 \pm 0.015 \\
0 \pm 0.013 \pm 0.0 \\
86 \pm 0.0085 \pm 0\end{array}$ & $\begin{array}{l}2^{\dagger}(\mathrm{BR}) \\
0109^{\dagger}(\mathrm{BR})\end{array}$ & $\begin{array}{l}{[38]} \\
{[39]} \\
{[40]}\end{array}$ \\
\hline $\mathrm{D}^{* \pm}$ & $\begin{array}{r}\text { ALEPH } \\
\text { DELPHI } \\
\text { OPAL }\end{array}$ & $\begin{array}{l}0.173 \pm 0.0 \\
0.156 \pm 0.0 \\
0.183 \pm 0.0\end{array}$ & $\begin{array}{l}4 \pm 0.007 \\
1 \pm 0.010 \pm 0.0 \\
9 \pm 0.007 \pm 0.0\end{array}$ & $\begin{array}{l}7^{\dagger}(\mathrm{BR}) \\
8\end{array}$ & $\begin{array}{l}{[38]} \\
{[39]} \\
{[41]}\end{array}$ \\
\hline $\mathrm{D}^{* * 0}$ & ALEPH & $\begin{array}{l}(1.02 \pm 0.26 \\
/ B R\left(\mathrm{D}^{* * 0} \rightarrow\right.\end{array}$ & $\begin{array}{l} \pm 0.14) \cdot 10^{-3} \\
\left.\mathrm{D}^{*+} \pi^{-}\right) / B R\left(\mathrm{D}^{*+}\right.\end{array}$ & $\left.\rightarrow \mathrm{D}^{0} \pi^{+}\right) / B R\left(\mathrm{~K}^{-} \pi^{+}\right.$ & $\begin{array}{l}{[38]} \\
+5\end{array}$ \\
\hline $\mathrm{D}_{\mathrm{s}}^{+}$ & $\begin{array}{r}\text { DELPHI } \\
\text { OPAL }\end{array}$ & $\begin{array}{l}0.088 \pm 0.0 \\
=B R(\mathrm{~b} \rightarrow \overline{\mathrm{B}} \\
0.128 \pm 0.0 \\
=B R(\mathrm{c} \rightarrow \mathrm{D} \\
\text { excl. decay } \\
0.00039 \pm 0 \\
=B R(\mathrm{~b} \rightarrow \mathrm{B}\end{array}$ & $\begin{array}{l}0 \pm 0.020 \\
\cdot B R\left(\overline{\mathrm{B}}_{\mathrm{s}}^{0} \rightarrow \mathrm{D}_{\mathrm{s}}^{+}\right) \\
9+0.019-0.01 \\
) \\
; \text { incl. } \mathrm{D}_{\mathrm{s}}^{+} \mathrm{h} \\
00011 \pm 0.0000 \\
\cdot B R\left(\mathrm{~B}_{\mathrm{s}}^{0} \rightarrow \mathrm{D}_{\mathrm{s}}^{-} \mathrm{l}\right.\end{array}$ & 6 & $\begin{array}{l}{[43]} \\
{[44]} \\
-)\end{array}$ \\
\hline & OPAL & $\begin{array}{l}0.322 \pm 0.0 \\
/ B R\left(\Lambda_{c}^{+} \rightarrow \mathrm{I}\right.\end{array}$ & $\begin{array}{l}8 \pm 0.038 \\
\left.\mathrm{~K}^{-} \pi^{+}\right)\end{array}$ & & [49] \\
\hline
\end{tabular}

${ }^{\dagger}$ : corrected with new branching ratio.

Table 6.8: Properties of charmed hadrons and their measured average multiplicities. 
Charmed mesons are important for studies of flavour specific properties of hadron production. Charm production in the fragmentation process is suppressed, as pointed out in Section 2.3, because of a negligible tunneling probability. Half of the direct charmed mesons originate from the primary quarks generated in $Z \rightarrow c \bar{c}$ events, the other half from $\mathrm{Z} \rightarrow \mathrm{b} \overline{\mathrm{b}}$ events with subsequent B-hadron decay. Only a small fraction arises from gluon splitting into a heavy quark pair. Identified charmed mesons are then used for flavour tagging of jets: the forward-backward asymmetry for c-quarks has been measured and the hadronic partial widths for charm and bottom determined [68].

The $\mathrm{D}^{0}$ signal is extracted by combining oppositely charged tracks. A cut on the decay angle $\cos \theta^{*}$ is applied thus rejecting the combinatorial background, which is peaked at small angles. In general no particle identification is used. Therefore the rate has to be corrected for double counting $(\approx 20 \%)$, when both choices of mass assignment enter into the signal peak.

Combining a $\mathrm{D}^{0}$ with a slow charged pion (denoted $\pi_{\mathrm{s}}^{ \pm}$), with the same charge as the $\mathrm{K}^{ \pm}$from the $\mathrm{D}^{0}$ decay, a $\mathrm{D}^{* \pm}$ signal is found. The momentum of the $\pi_{\mathrm{s}}^{ \pm}$may be restricted to the kinematically allowed range for combinatorial background rejection, and a clear signal is seen in the mass difference $m\left(\mathrm{~K}^{ \pm} \pi^{\mp} \pi_{\mathrm{s}}^{ \pm}\right)-m\left(\mathrm{~K}^{ \pm} \pi^{\mp}\right)$. The collaborations have studied the background with the event mixing technique, i.e., combining the two pions with the kaon from another event, or by track mixing, i.e., combing the $\mathrm{D}^{0}$ tracks with a $\pi_{\mathrm{s}}^{ \pm}$reflected from the opposite hemisphere; selecting fake $\mathrm{D}^{0}$ mesons from like-sign combinations is another choice.

The $\mathrm{D}^{ \pm}$is detected in the decay $\mathrm{D}^{ \pm} \rightarrow \mathrm{K}^{\mp} \pi^{ \pm} \pi^{ \pm}$. The invariant mass of three charged particles is calculated, where the two particles with the same sign get the pion mass assigned, the third particle is taken as the kaon. Cuts on the flight direction of the $\mathrm{D}^{ \pm}$ improve the signal to background ratio.

Evidence for $D^{* *}$ is found by one experiment (ALEPH), when a $D^{* \pm}$ is combined with a charged pion to form a $\mathrm{D}^{* * 0}$ with high momentum $\left(x_{E}>0.25\right) .63 \pm 16$ events are seen.

The studies take into account the differences in efficiency for charmed hadrons originating from $\mathrm{c}$ - or b-decays, because of the harder fragmentation of bottom quarks and the different charmed meson spectrum. This is expected from independent studies at and around the $\Upsilon(4 \mathrm{~S})$ resonance [114]. Therefore, separation of $c \bar{c}$ and $b \bar{b}$ events is performed using the following strategies: estimators for the event to be $b \bar{b}$ or $c \bar{c}$ from event shape variables are formed, which are independent of the D-meson or calculated from the hemisphere opposite to the D; leptons with high transverse momentum to the jet are used; lifetime as measured in the opposite hemisphere is taken as criterion; the lifetime of the $D$ itself is used.

The $\mathrm{D}_{\mathrm{s}}$ has been seen in many decay modes, but no inclusive production rate has been given by the experiments. This meson was mainly used in $\mathrm{D}_{\mathrm{s}}$ lepton correlation studies for the extraction of the $B_{s}$ meson signal and the $B_{s}$ lifetime measurement. The consistency of the extracted $\mathrm{D}_{\mathrm{s}}$ momentum spectrum with the simulation has been checked.

The $\Lambda_{c}^{+}$is the only charmed baryon that is produced copiously enough for detection. For the detection in the decay $\Lambda_{c}^{+} \rightarrow \mathrm{pK}^{-} \pi^{+}$all three particles are identified using the specific energy loss. They should also be consistent with one common vertex which must be well separated from the collision point for background suppression. If the invariant 
mass of the proton and kaon, but assigning the kaon mass to both particles, is consistent with the $\phi$ mass, the candidate is rejected: this suppresses background from $\mathrm{D}_{\mathrm{s}}^{+} \rightarrow \phi \pi^{+}$ decays. The differences in the $x_{E}$ spectra are used for the separation of the $c$ - and bcontribution.

\section{Momentum Spectra}

The inclusive distribution of the $\mathrm{D}^{* \pm}$ is shown in Figure 6.14. The JETsET and HERwIG models agree with the experimental results. The $\mathrm{D}_{\mathrm{s}}^{+}$momentum spectrum was measured in various decay channels. Taking the low statistical significance into account, the distributions of the simulation are in agreement with the experiments (Fig. 6.15).

The investigation of inclusive charmed meson production has concentrated on the inclusive charm fragmentation function [115]. The fragmentation function is parameterized with the Peterson fragmentation function; the result is expressed as the average momentum of the charm quark $\left\langle x_{E, c}\right\rangle$.

Besides the measurement of the $D^{* \pm}$ decay, one must separate the direct production of charmed mesons from the indirect production, i.e., from b-decay. This is done with various Monte Carlo studies, which use the different event characteristics of b- and cevents. A subtraction of the contribution from b-decay can be done using data with a $\mathrm{D}^{* \pm}$ in $\mathrm{B} \rightarrow 1^{-} \nu \mathrm{X}$ events, identified by the lepton and missing energy, because the neutrino is not seen in the detector. Lifetime information was used, or the $\mathrm{D}^{0}$ decay length distribution was investigated, which depends on the flavour composition of the event. Finally, $\left\langle x_{E, c}\right\rangle$ may be corrected for the contribution from gluon splitting, which is a small effect of $\approx 2 \%$. The results are shown in Figure 6.16 [115] with an average of

$$
<x_{E, c}>=0.499 \pm 0.005 \pm 0.005
$$

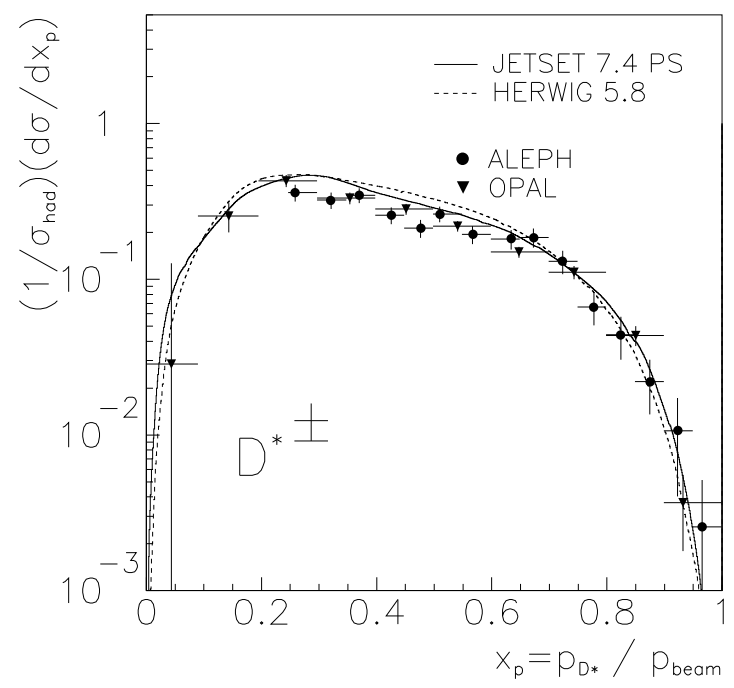

Figure 6.14: Momentum spectrum for $D^{\star \pm}$. 

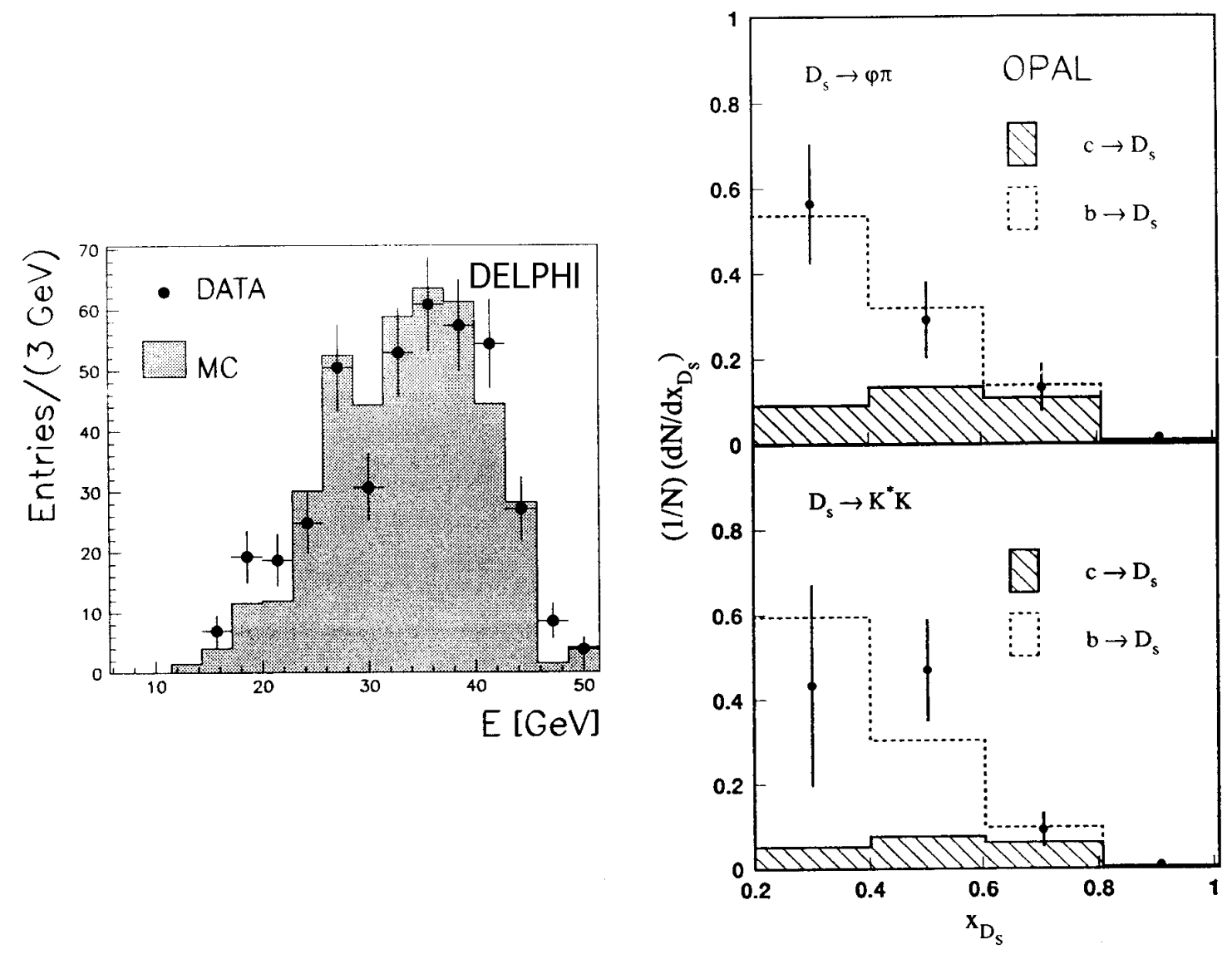

Figure 6.15: Momentum spectrum for $D_{\mathrm{s}}^{+}$of DELPHI [43] (from the $D_{\mathrm{s}}^{+} h$ analysis) and OPAL [44] data in comparison with JETSET model. 


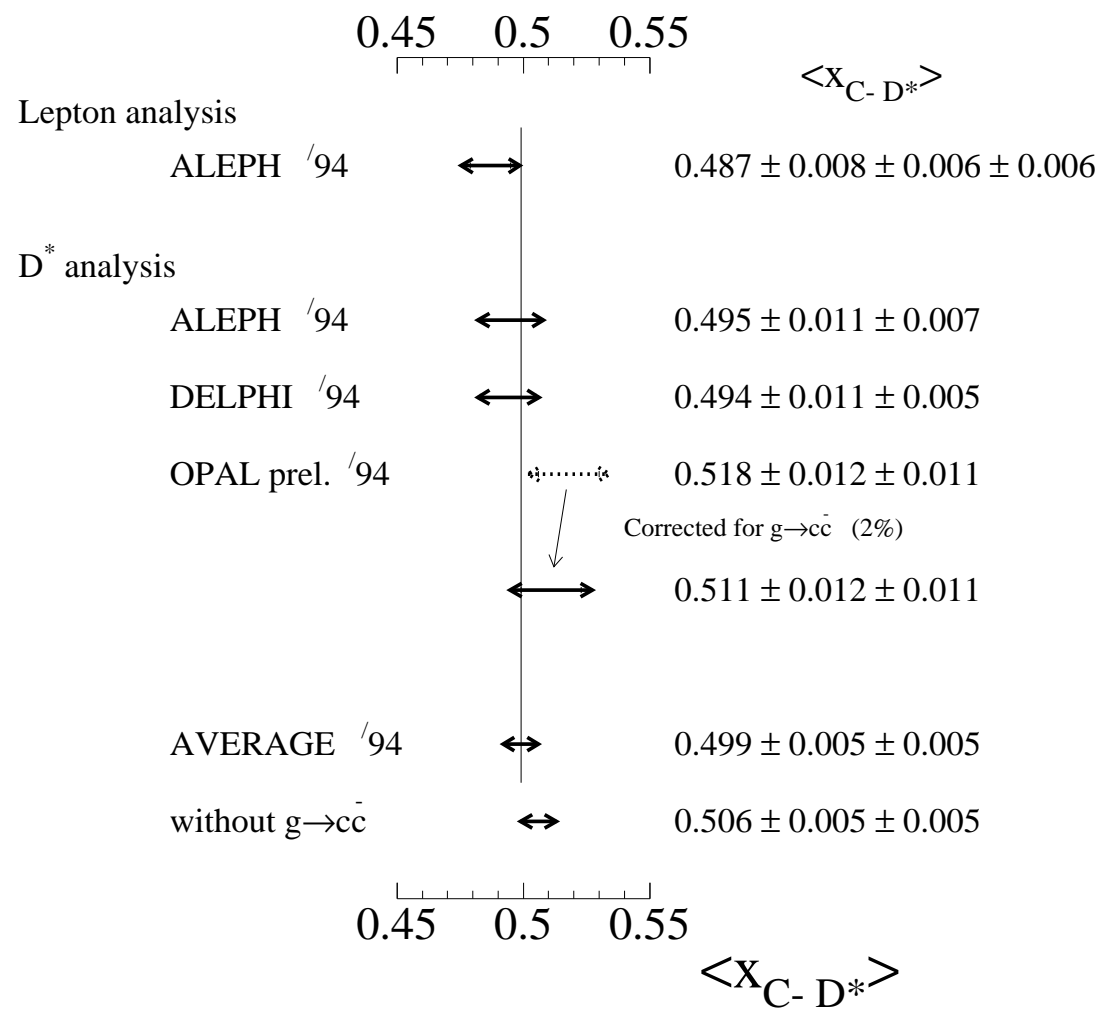

Figure 6.16: Measurements of $\left\langle x_{E, c}\right\rangle$ for charm quarks. The average is given including the contribution from gluon splitting and without this contribution [115]. 


\subsection{Charmonia and Bottomonia}

Extraction of the $\mathbf{J} / \psi, \psi^{\prime}, \chi_{c}$, and $\Upsilon$ Signals

\begin{tabular}{|c|c|c|c|c|c|}
\hline \multicolumn{2}{|c|}{ Particle } & $\begin{array}{c}\text { Mass } \\
{\left[\mathrm{MeV} / \mathrm{c}^{2}\right]}\end{array}$ & $\begin{array}{c}\text { Width } \\
{\left[\mathrm{MeV} / \mathrm{c}^{2}\right]}\end{array}$ & Decay mode & $\begin{array}{l}\mathrm{BR} \\
{[\%]}\end{array}$ \\
\hline$\overline{\mathrm{J} / \psi}$ & ADLO & 3097 & 0.088 & $\begin{array}{l}\mathrm{e}^{+} \mathrm{e}^{-} \\
\mu^{+} \mu^{-}\end{array}$ & $\begin{array}{l}5.99 \\
5.97\end{array}$ \\
\hline$\psi^{\prime}$ & $\mathrm{DO}$ & 3686 & 0.002 & $\begin{array}{l}\mathrm{J} / \psi \pi^{+} \pi^{-} \\
\hookrightarrow \mu^{+} \mu^{-}\end{array}$ & $\begin{array}{l}32.4 \\
5.97\end{array}$ \\
\hline$\chi_{c}$ & DL & 3511 & 0.88 & $\begin{array}{l}\mathrm{J} / \psi \gamma \\
\hookrightarrow \mu^{+} \mu^{-}\end{array}$ & $\begin{array}{l}27.3 \\
5.97\end{array}$ \\
\hline \multirow[t]{2}{*}{$\Upsilon, \Upsilon^{\prime}, \Upsilon^{\prime \prime \ddagger}$} & $\mathrm{O}$ & 9460 & 0.053 & $\mathrm{e}^{+} \mathrm{e}^{-}, \mu^{+} \mu^{-}$ & 2.31 \\
\hline & & Multiplici & y/Z-decay ${ }^{\dagger}$ & & Ref. \\
\hline \multirow[t]{4}{*}{$\overline{\mathrm{J} / \psi}$} & ALEPH & 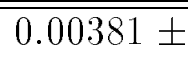 & $\overline{0.00041 \pm 0}$ & .00026 & {$[45]$} \\
\hline & DELPHI & $0.00373 \pm$ & $0.00039 \pm 0$ & .00036 & {$[46]$} \\
\hline & L3 & $0.0036 \pm$ & $0.0005 \pm 0$ & .0004 & {$[47]$} \\
\hline & OPAL & $0.0039 \pm$ & $0.0002 \pm 0$ & .0003 & {$[48]$} \\
\hline \multirow[t]{2}{*}{$\psi^{\prime}$} & DELPHI & $0.00160 \pm$ & $0.00073 \pm 0$ & .00073 & {$[46]$} \\
\hline & OPAL & $0.0016 \pm$ & $0.0003 \pm 0$ & .0002 & {$[48]$} \\
\hline \multirow[t]{2}{*}{$\chi_{c}$} & DELPHI & $0.00 .50 \pm$ & $0.0021+0$ & $.0015-0.0009$ & {$[46]$} \\
\hline & L3 & $0.0075 \pm$ & $0.0029 \pm 0$ & .0006 & {$[47]$} \\
\hline$\Upsilon, \Upsilon^{\prime}, \Upsilon^{\prime \prime \ddagger}$ & OPAL & $0.00010 \pm$ & $0.00004 \pm 0$ & $.00001 \pm 0.00002$ & {$[56]$} \\
\hline
\end{tabular}

†: The multiplicities are given as branching ratio of the $\mathrm{Z}$ rather than per hadronic event. The collaborations used a leptonic branching ratio of the $\mathrm{J} / \psi$ of $5.91 \%$ [116] rather than $5.98 \%$ [79].

$\ddagger$ : For the $\Upsilon$ an effective branching ratio was used, assuming relative production rates 1:0.5:0.5 for $\Upsilon: \Upsilon^{\prime}: \Upsilon^{\prime \prime}$, taking cascade decays into account.

The last errors to the production rate give the uncertainty from the $\Upsilon$ production mechanism.

Table 6.9: Properties of charmonia and bottomonia and their measured average multiplicities.

The production of $\mathrm{J} / \psi$ in hadronic events is a clear evidence for the production of bottomhadrons. The $c \bar{c}$ is unlikely to be produced in the fragmentation process: only a few percent of the charmonia are from soft gluon emission and fragmentation. Some $\mathrm{J} / \psi$ come from the decay of $\psi^{\prime}$ and $\chi_{c}$. Only $\chi_{c 1}$ has been detected, but neither $\chi_{c 0}$ nor $\chi_{c 2}$, which agrees with theoretical considerations that $\chi_{c 0}$ and $\chi_{c 2}$ may not come from b-decays [117]. With the $\mathrm{J} / \psi$ detected in its leptonic decay modes $\left(\mathrm{e}^{+} \mathrm{e}^{-}\right.$and $\left.\mu^{+} \mu^{-}\right)$, the vertex of the two leptons is a clear signature. The lifetime of B-hadrons has been measured and exclusive decays investigated, or limits on their branching ratios given.

Two leptons with opposite charge are selected with a minimum momentum (typically $2.5 \mathrm{GeV} / c$ ) for good lepton identification, consistent with a common vertex. The signal 
to background ratio is improved by additional cuts on the $\mathrm{J} / \psi$ momentum or requiring the two leptons to be emitted into the same hemisphere. The main background from cascade decays of the b-quark $(\mathrm{b} \rightarrow \mathrm{cl} \bar{\nu}$ followed by $\mathrm{c} \rightarrow \mathrm{s} \bar{l} \nu)$ is rejected by the missing energy in the $\mathrm{J} / \psi$-hemisphere: The requirement that the visible energy in the hemisphere must exceed $80 \%$ of the beam energy rejects most of the background events, in which the energy of the two neutrinos escaped detection.

The signal is extracted from a fit to the di-lepton mass distribution: a Gaussian for the signal (electrons are corrected for bremsstrahlung, when the photon is detected) and a background function. The $\mathrm{e}^{ \pm} \mu^{\mp}$ spectrum and the like-sign mass spectrum are used as check or for background parameterization.

The $\psi^{\prime}$ is reconstructed by combining a $\mathrm{J} / \psi$ in the muonic decay channel with a $\pi^{+} \pi^{-}$pair of invariant mass $m\left(\pi^{+}, \pi^{-}\right)>0.4 \mathrm{GeV} / c^{2}$. The particle trajectories must be consistent with the $\mathrm{J} / \psi$ vertex.

For the $\chi_{c}$ the $\mathrm{J} / \psi$ with muonic decay is combined with a photon, which may not be a photon from a $\pi^{0}$ candidate.

The interest in the $\Upsilon\left(=\Upsilon, \Upsilon^{\prime}\right.$, and $\left.\Upsilon^{\prime \prime}\right)$, which should have a small production rate because of its high mass, was initiated by the high rate measured at the Tevatron p $\bar{p}$ collider [118]. The CDF result may find its explanation in a new model, which allows, besides colour-singlet production, a colour-octet production [119]. The theoretical Zbranching ratio of the three $\Upsilon$ states is expected to $5.9 \cdot 10^{-5}$.

The OPAL collaboration has observed an $\Upsilon$ signal measured in the electron and muon decay channels. Beside standard selection criteria for these leptons, an isolation cut is used reducing the hadronic event background. The remaining background is: fake leptons (misidentified hadrons) and leptons from heavy flavour hadrons, which can be estimated using $e^{ \pm} \mu^{\mp}$ pairs; four fermion events $\left(\mathrm{e}^{+} \mathrm{e}^{-} \rightarrow \mathrm{q} \overline{\mathrm{q}}+1^{+} 1^{-}\right)$, which is estimated using simulated events. In 3.7 million events, 8 candidates are found with $1.6 \pm 0.3$ background events, i.e., $6.4 \pm 2.8 \pm 0.3 \Upsilon$ candidates. To obtain the inclusive cross section the uncertainty on the production mechanism was included.

The numbers in publications are given as branching ratios of the $Z$. Therefore, the published data are corrected by $\Gamma_{\text {had }} / \Gamma_{\text {tot }}$ for Table 5.1 in Section 5.2.

\section{Momentum Spectra}

The extracted production rates are in reasonable agreement between the experiments; the models do well, if the particle production is allowed at all, except for the $\chi_{c}$. The published spectra only of the $\mathrm{J} / \psi$ are given showing the data with the JETSET model. The momentum spectrum of the $\mathrm{J} / \psi$, which is harder than for the light hadrons, is due to its origin from b-decays. The spectrum is almost symmetric around $x_{E}=0.5$ and compares well with the model prediction. The measured production rate of the $\Upsilon$ is in agreement with the model including both colour-singlet and colour-octet production, where the latter is the dominant source of the $\Upsilon$, which shows the need for this contribution. The size of the OPAL signal is consistent with upper limits given by the DELPHI collaboration [120].

There is much room left for further investigations. Some experiments have not looked 
at all the charmonia and bottomonia; other decay channels should be studied for an improved combined inclusive production rate. A better signal would be useful for studies of gluon-jet and gluon-splitting (see Section 7.5).
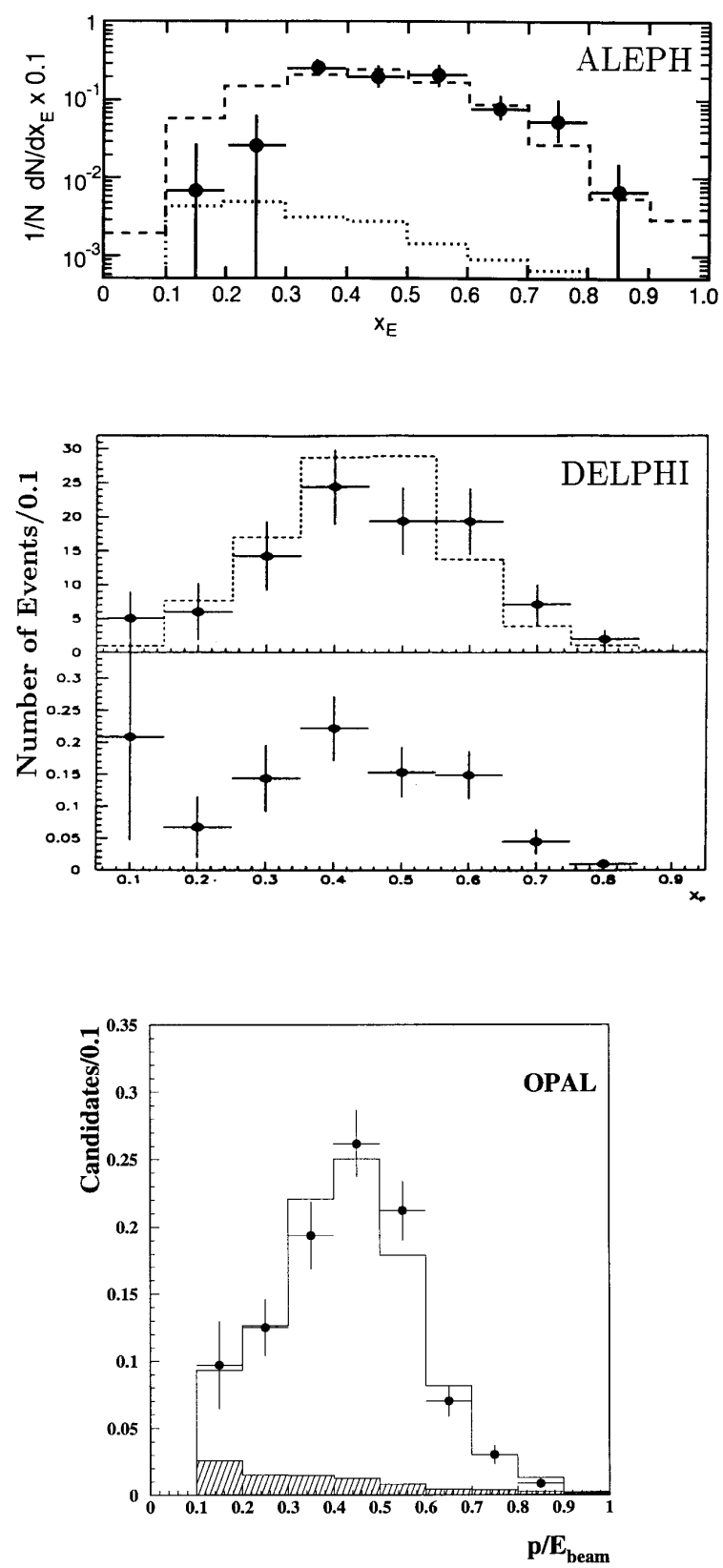

Figure 6.17: Momentum spectra for $J / \psi$ of ALEPH [45] (the dashed and dotted line indicates $J / \psi$ originating from $b \bar{b}$ and gluon, respectively; the prediction is normalized to the data), DELPHI [46] (the upper plot is without detector acceptance correction), and OPAL [48] (the hatched area shows the contribution from the fragmentation) data in comparison with JETSET model. 


\subsection{Bottom Hadrons}

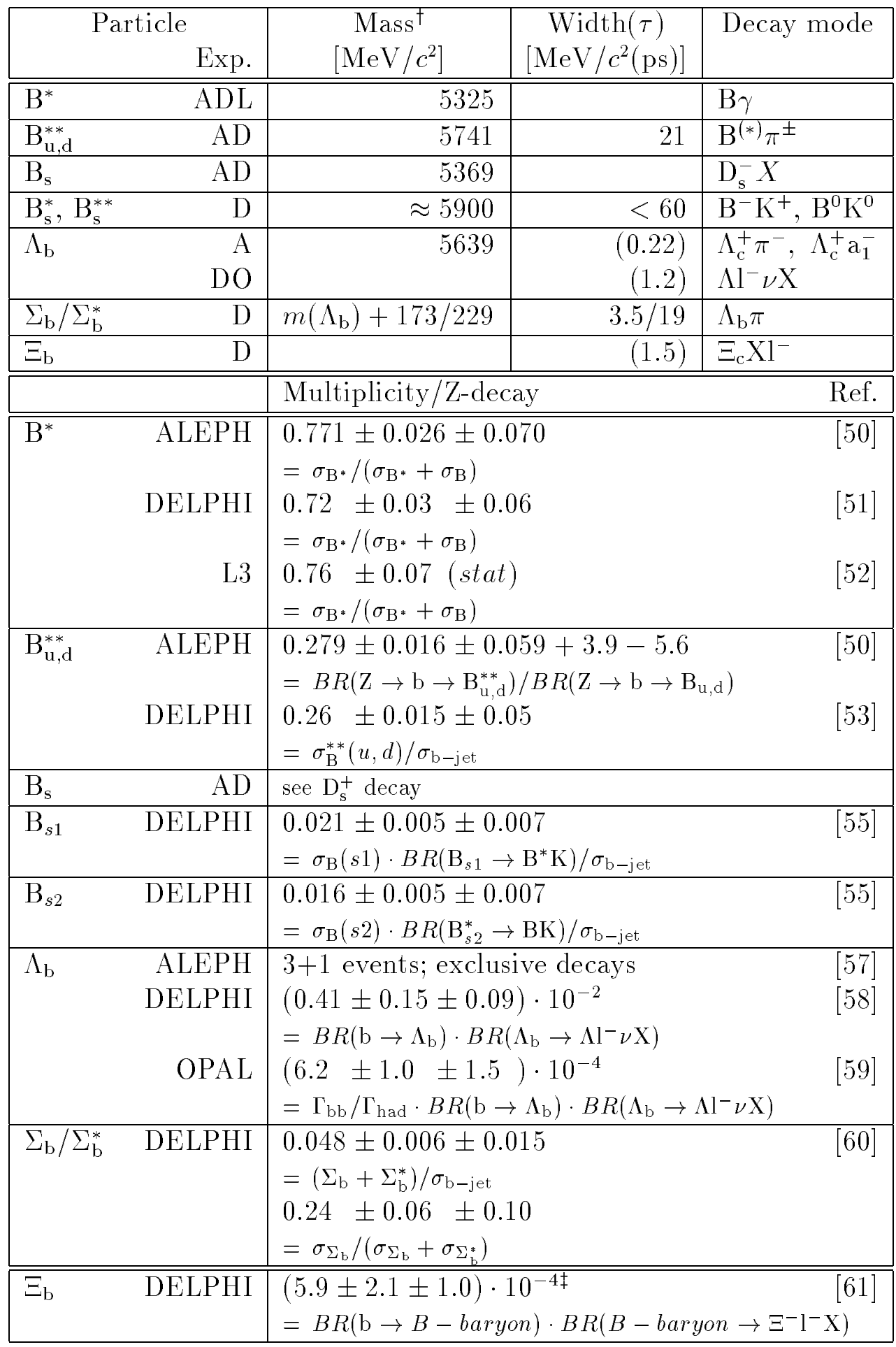

†: Masses of the b-hadron are partially quite uncertain. The mass values are taken from Refs. [79] or [122] or taken from the LEP experiment, that measured the production.

$\ddagger$ : The major part of B-baryons are $\Xi_{\mathrm{b}}$ with a small contribution from $\Lambda_{\mathrm{b}}(<10 \%)$.

Table 6.10: Properties of bottom hadrons and their measured average multiplicities. 
About $22 \%$ of the hadronic decays of the $Z$ are $b \bar{b}$ events. With several million events, at LEP, aspects of b-physics similar and complementary to those at B-factories are studied. Primary goals are precision measurements of the partial width of the $Z$ decay into a $b \bar{b}$ pair, $\Gamma_{\mathrm{b} \overline{\mathrm{b}}}$, and the forward-backward asymmetry in these decays. In B-hadron physics the determination of $\mathrm{B} \overline{\mathrm{B}}$ mixing, lifetimes, mass measurements, search for new particles and decays, constraints on the CKM-matrix etc. are of high priority.

B-hadrons have been identified, as listed in Table 6.10, and production rates have been extracted. The B-hadron spectroscopy made progress especially in the sector of excited B-mesons, where inclusive momentum spectra have been measured (see Reference [122] for a recent review). The measured production of excited states is high, $\mathrm{B}^{*} /\left(\mathrm{B}^{*}+\mathrm{B}\right)=$ $0.75 \pm 0.04$ and agrees with the expectation from heavy quark effective theory (HQET) [123] using spin counting, which gives the probability for the production of spin 1 (vector) states compared to pseudoscalar plus vector states as $[\mathrm{V} /(\mathrm{V}+\mathrm{P})]_{\mathrm{b}}=0.75$. The same is found for $\Sigma_{\mathrm{b}}$.

As for the charmed hadrons, in their study of b-hadron fragmentation the LEP collaboration have concentrated on the inclusive b-quark fragmentation function [115]. Figure 6.18 shows the agreement of the measurements with b-fragmentation functions. For the inclusive theoretical b-fragmentation measurements, b-hadrons are tagged (see as well in Section 7.4 below) using high $p_{t}$ leptons, which requires a model for the semi-leptonic decay. The mean of $x_{E, b}$ is obtained from the momentum of the lepton ( $p$ and $p_{t}$ to the thrust axis). When charmed mesons are used for the $\left\langle x_{E, b}\right\rangle$ measurement, one needs measurements of the momentum of the B-hadron decay products, including the neutrino momentum. The latter can be determined by a simultaneous measurement of the visible energy measured in the hemisphere and the invariant mass in the two hemispheres. Other studies have used the distance of the primary to the secondary vertex or $\mathrm{J} / \psi$ mesons.

In Figure 6.19 the measurements are summarized [115], where an average of

$$
\left.<x_{E, b}\right\rangle=0.701 \pm 0.002 \pm 0.009
$$

is obtained. 

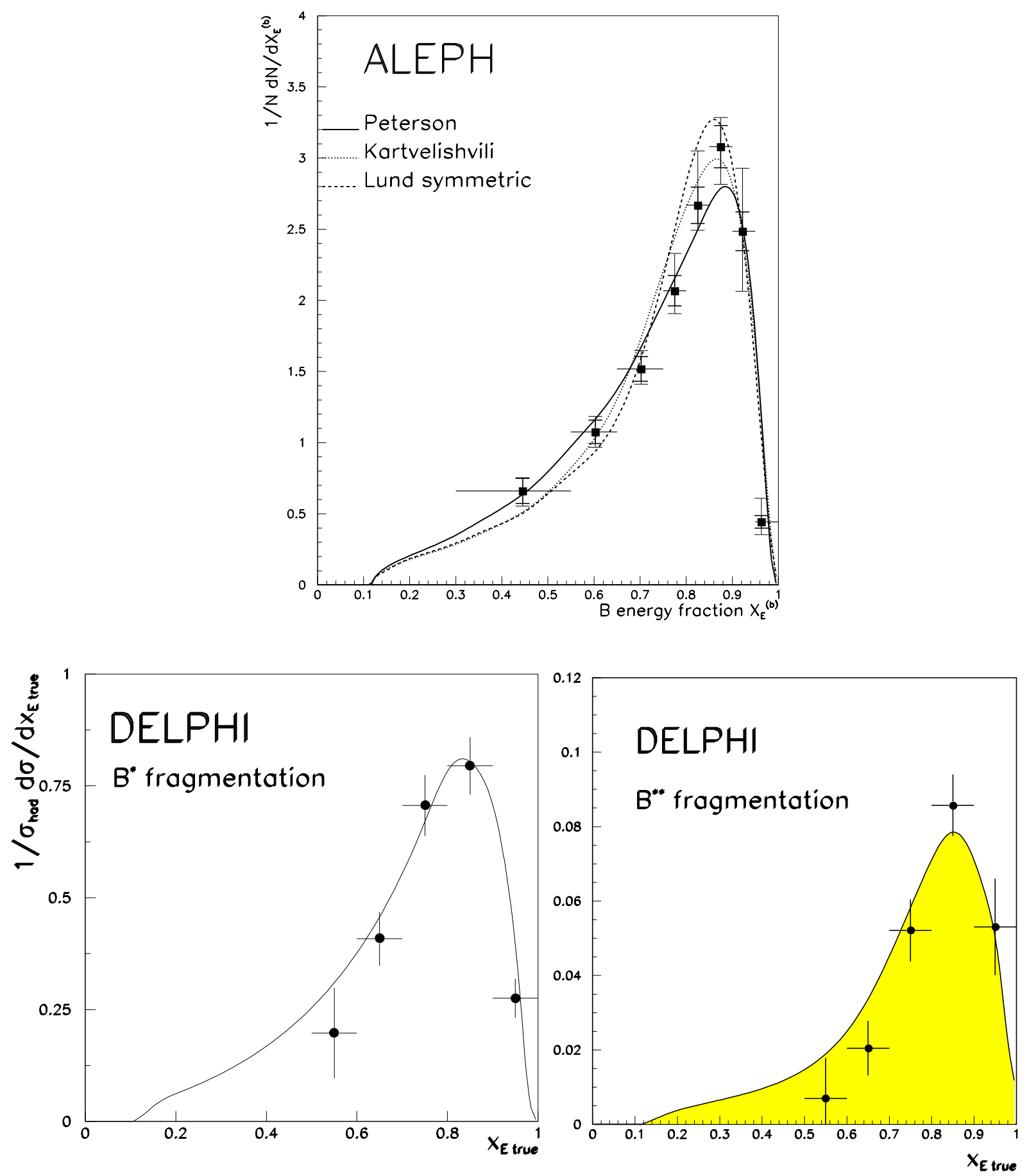

Figure 6.18: Momentum spectra for the leading b-hadrons of ALEPH [121] data in comparison with different fragmentation functions; for $B^{*}$ and $B^{* *}$ of DELPHI data [51, 53] in comparison with JETSET model (with Peterson fragmentation function). 


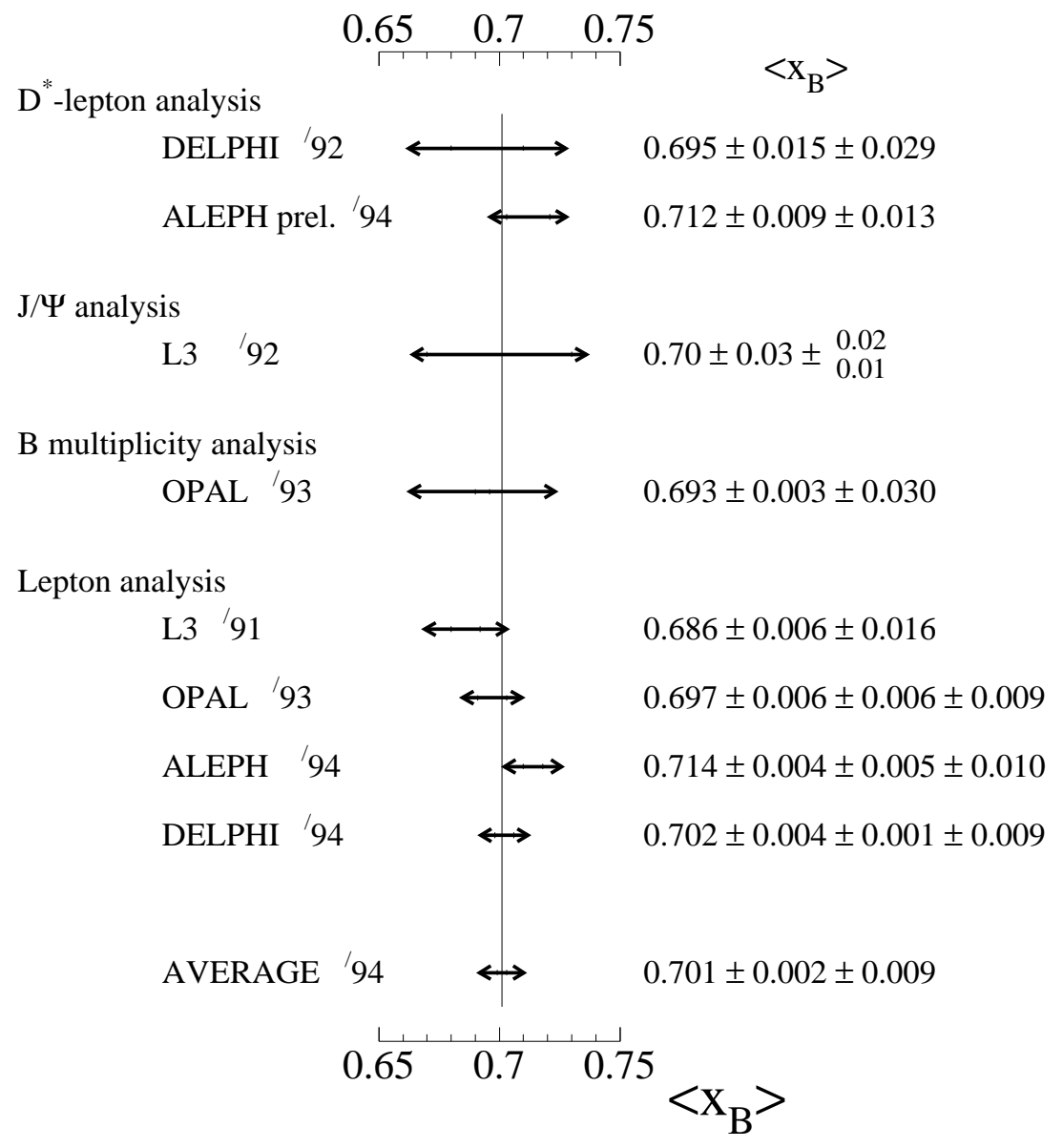

Figure 6.19: Measurements of $\left\langle x_{E, b}\right\rangle$ for bottom quarks [115]. 


\section{Chapter 7}

\section{Related Results}

\subsection{Comparisons with Models}

Spin-Counting, Isospin

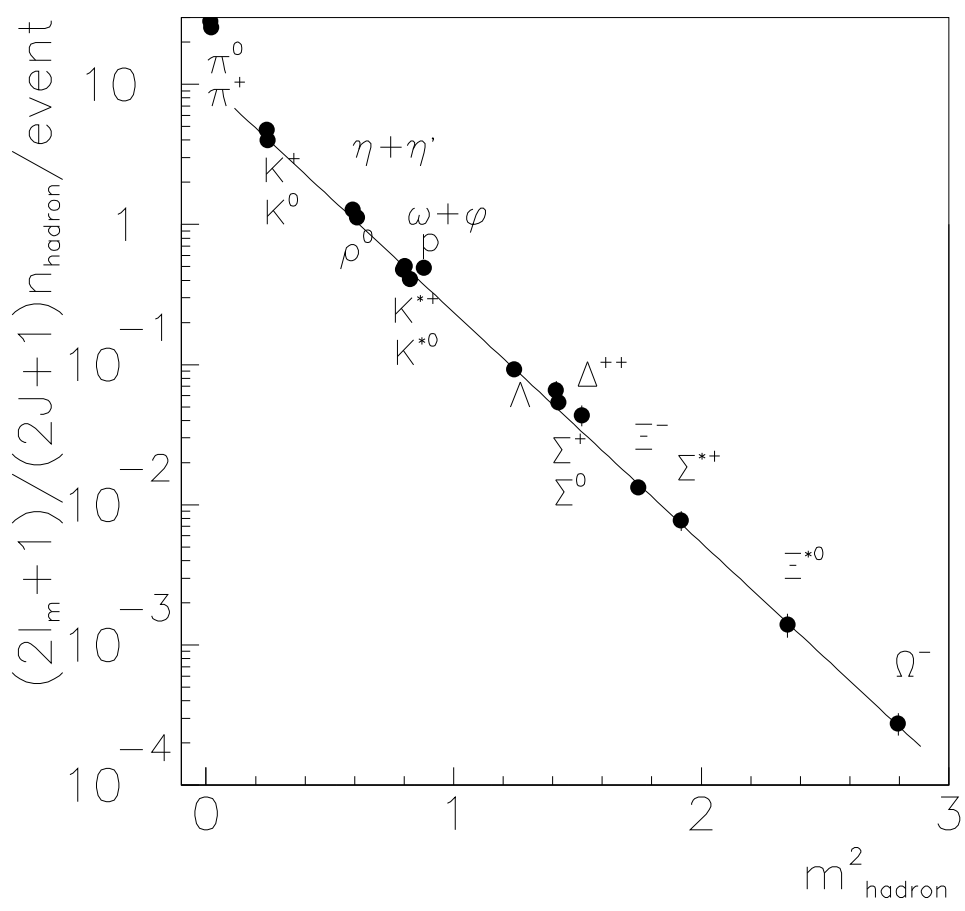

Figure 7.1: The Chliapnikov-model; $\langle n\rangle$ is the particle multiplicity without counting the anti-particle.

Though the model of P.V. Chliapnikov and V.A. Uvarov is a purely phenomenological model for a description of the average multiplicity of particles of different kind per hadronic 
Z decay as function of spin $J$ and modified isospin $I_{m}$, is describes successfully the data from the LEP experiments (if one excludes the pions). The fit gives a $\chi^{2} / n d f=2$ (Figure 7.1): the parameters $a$ and $b$ in the function

$$
<n>=\frac{2 J+1}{2 I_{m}+1} a \exp \left(-b M^{2}\right)
$$

take the values $10.4 \pm 0.2$ and $3.78 \pm 0.02\left(\mathrm{GeV} / c^{2}\right)^{-2}$ respectively. $(\langle n\rangle$ is the particle multiplicity without counting the anti-particle.) The data from PEP and PETRA experiments around $\sqrt{s} \approx 35 \mathrm{GeV}$ are described by $a=8.0 \pm 0.4$ and $b=3.97 \pm 0.05\left(\mathrm{GeV} / c^{2}\right)^{-2}$ [82]; the slope $b$ is compatible for both energies and $1 / \sqrt{b}=513 \pm 2 \mathrm{MeV} / c^{2}$ is close to the kaon mass, which may be a pure coincidence; whether the pion, which is not on the fitted curve (It predominately stems from decays and may originate from the QCD vacuum, because it is a Goldstone boson [82]) indicates a problem in the model or something new is not obvious.

Although the modified isospin $I_{m}$ may look ad hoc; the factor $2 J+1$ shows that the spin counting factor and the mass squared play an important role. The physics insight into QCD, however, is unclear.

Also worth mentioning is the UCLA model [124], which is a modification of the JETSET model, using its parton shower and hadron decay tables but replacing its fragmentation function. The fragmentation of the UCLA model is deduced using the area in the spacetime diagram (see Figure 2.7 for a very simple example of such a diagram), avoiding the need of the many suppression parameters in the JETSET model. It turns out that the particle production is proportional to $\exp \left(-b M^{2}\right)$, the same factor used in the parameterization used by P.V. Chliapnikov and V.A. Uvarov.

The geometrical two-chain model (G2C) [125] is another model with a similar mass suppression factor $\exp \left(-\pi\left(m_{h}-m_{l}\right)^{2} / \kappa\right) ; m_{h}$ is the hadron mass and $m_{l}$ is the lightest hadron containing the particular quark combination involved [126]. However, it uses further suppression factors for diquark production, for $L=1$ production probability of diquarks and of light mesons. The G2C model, originally developed as fast and accurate Monte Carlo generator for cosmic ray extensive air showers, describes many of the particle rates in $\mathrm{e}^{+} \mathrm{e}^{-}$annihilation [127].

Furthermore, a simple phenomenological approach has been discussed in Ref. [128], where the production rate is related to the binding energy $E_{b i n d}=m_{h}-\sum_{i} m_{q i}$ of the hadron with mass $m_{h}$ which is composed of quarks with mass $m_{q i}$.

\section{Thermodynamical Model}

Figures 7.2 and 7.3 show that the thermodynamical approach by F. Becattini [83], tuned to LEP data available by mid 1995, describes the new average production rates quite well (see Section 2.4.3). The results of the fit are given in Table 7.1 for the correlated jet scheme, the scheme where quantum numbers are conserved for the event, but not necessarily within each jet. The results for the uncorrelated one are comparable. The model predicts rates for the heavy flavour particles as well, and are found in agreement with the data. The value of the temperature is interestingly close to the QCD parameter 


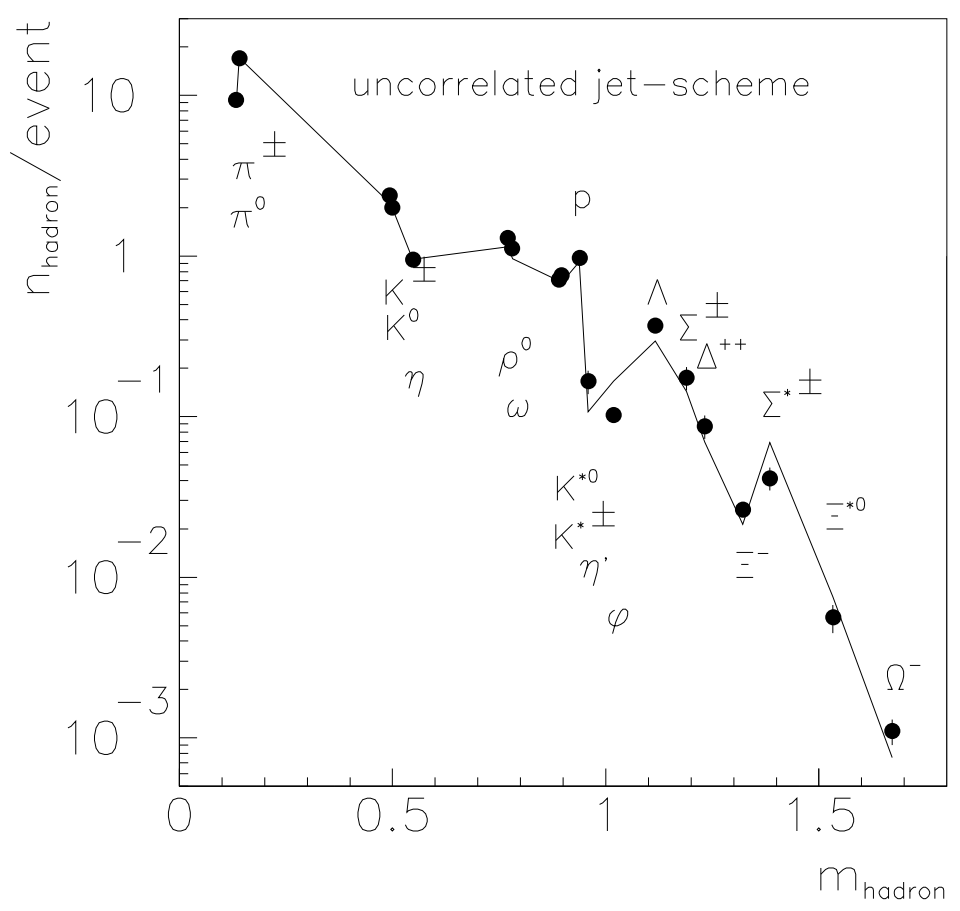

Figure 7.2: Particle multiplicity in the thermodynamical model: data are compared with the uncorrelated jet scheme.

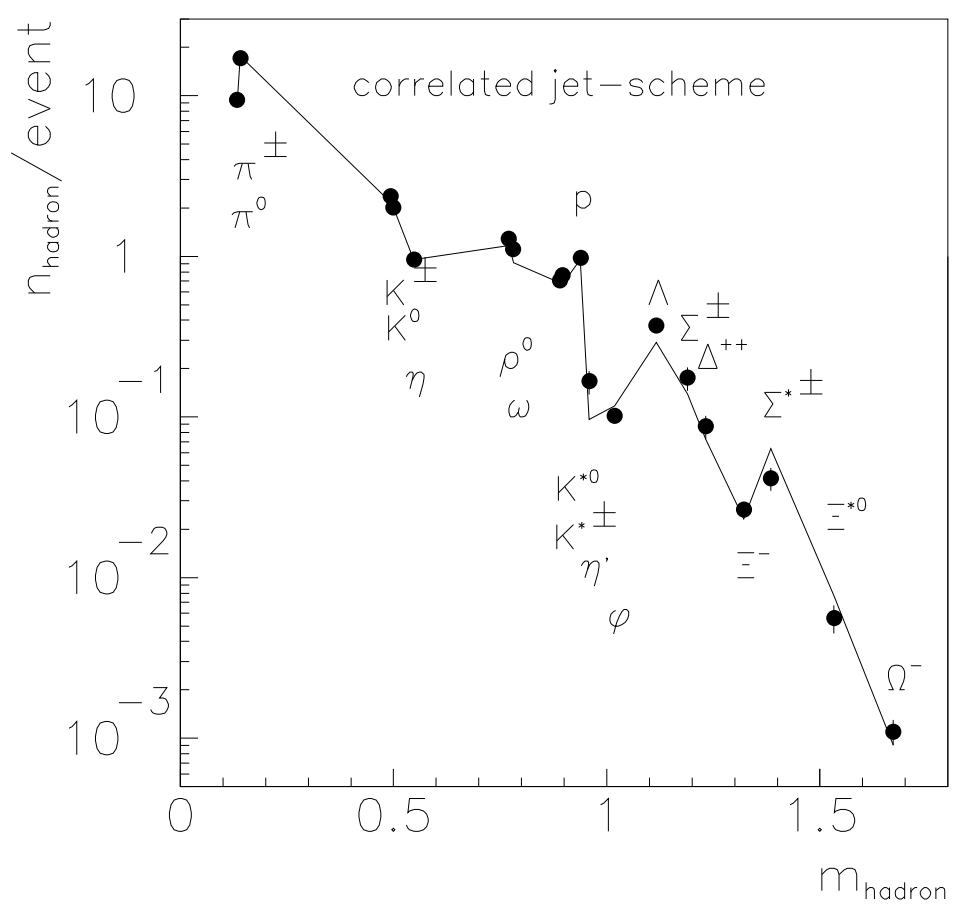

Figure 7.3: Particle multiplicity in the thermodynamical model: data are compared with the correlated jet scheme. 


\begin{tabular}{|c|c|c|}
\hline Parameter & $\sqrt{s}=35 \mathrm{GeV}$ & $\sqrt{s}=91 \mathrm{GeV}$ \\
\hline \hline Temperature $(\mathrm{MeV})$ & $167.0 \pm 3.0$ & $156.5 \pm 1.8$ \\
\hline Volume $\left(\mathrm{fm}^{-3}\right)$ & $10.41 \pm 1.5$ & $28.1 \pm 2.5$ \\
\hline$\gamma_{\mathrm{s}}$ & $0.769 \pm 0.05$ & $0.690 \pm 0.032$ \\
\hline$\chi^{2} / n d f$ & 4.4 & 4.7 \\
\hline
\end{tabular}

Table 7.1: Fitted parameter values at PEP/PETRA and LEP energies for the thermodynamical approach in the correlated jet scheme [83].

$\Lambda_{Q C D}$. The volume is rather large and a point of criticism. One would expect an increase in temperature from PEP/PETRA data to LEP data; the fits show the opposite trend. A similar argumentation holds for the strangeness suppression $\gamma_{s}$. An improvement of the model is needed which must include multi-jet events. A third jet would change the scenario. An explanation, how the equilibrium is reached is not given. The question, what is chance, what is physics needs certainly to be answered [108].

\subsection{Relative Rates}

\section{a) Baryon Suppression}

From the multiplicities of identified particles measured at LEP and collected in Table 5.2 it is obvious that baryon production is lower than meson production. A comparison of charged pion and proton rates and spectra, both copiously produced and composed of the light $u$ and $d$ quarks, reveal large differences (see Reference [106] and references therein).

Mesons consist of a quark and an anti-quark; in the fragmentation process mesons are formed from a quark and an anti-quark from the vacuum. For baryons, made from three quarks, various production mechanisms may be considered [106] (Figure 7.4). Baryons may be thought as being formed from three quarks at random. It turns out that correlations between the constituent quarks are important for baryon production (see Section 7.7). Two quarks are combined in a ground state with spin 0 or 1 , called diquark. The diquark production is suppressed, because the mass is higher than that of a single quark. The tunneling probability, an argument used in the JETSET model, is lower. The mass assignment for a diquarks is difficult; models generally have a free parameter for the suppression of diquark production.

An anti-diquark is created simultaneously with the diquark and combined with an antiquark or quark. Quantum numbers have to be conserved locally; consequently, baryons are adjacent in phase space. Therefore, baryons and anti-baryons are expected in the same jet (local baryon number conservation). This strict correlation can be softened by meson creation in between the baryons. This so-called popcorn mechanism is favoured by experimental data on baryon correlations. The probability for production of an intermediate meson between two baryons is left as a free parameter in the JETSET model. No possibility for more intermediate mesons is given in the model. 
The baryon production in the cluster model HERWIG is allowed through the splitting of a gluon into a diquark anti-diquark pair at the end of the parton shower. Baryons are suppressed by their heavier mass, reducing the available phase space in the cluster decay. Therefore baryon production is very sensitive to the maximum cluster mass.

In Figure 6.5 an example of the relative baryon to meson production was shown and the lower baryon production is obvious. In addition, the inclusive baryon spectra show that baryon spectra are not adequately described by the models, but the predictions are too hard. In JETSET this has been improved introducing the parameter for leading baryon suppression; HERWIG, however, has problems describing the shape of the momentum distributions, especially for $\mathrm{p}$ and $\Lambda$. The solution in JETSET (the suppression of the leading quarks) may be premature; both models do not include excited baryons, which may have an impact on the momentum distributions. The influence of orbitally excited particles was seen, when scalar and tensor mesons were introduced improving the description of light mesons, e.g., $\mathrm{K}^{ \pm}$and $\mathrm{K}^{*}$.

(a)

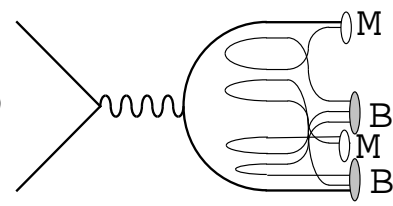

(b)

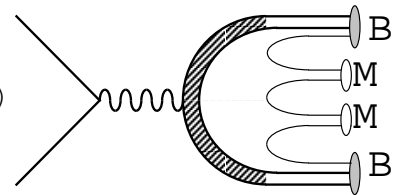

(c)

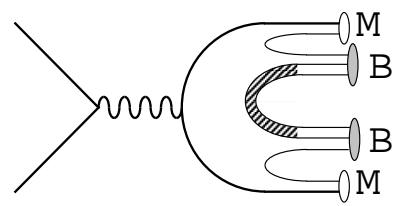

(d)

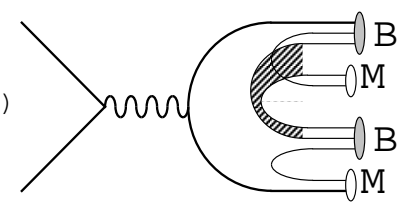

Figure 7.4: Different models for baryon production. a) recombination of quarks, b) leading diquarks, c) diquarks from fragmentation, d) diquarks with the popcorn mechanism [32].

\section{b) Strangeness Suppression}

The multiplicities and spectra reveal, besides the baryon suppression, a strong dependence on the flavour content of the particles. Strangeness suppression has received a lot of attention and has, for example, been studied in hadron-hadron interactions [129] and in heavy ion collisions [130], where strangeness enhancement might indicate the formation of quark-gluon plasma. The results from these experiments are similar to the values for the strangeness suppression discussed here, in events from $\mathrm{e}^{+} \mathrm{e}^{-}$annihilation. 
The idea of describing quark anti-quark production by a tunneling process suggests that particle production is related to the quark masses. Bottom quarks are expected to originate primarily from the leading quark; charm particles may come from bottom decay and from leading quarks. The light quarks are predominately produced in the breakup of the string. If the approach of tunneling in the JETSET model is confirmed by experiment, it shows that the production probability of light flavours is governed by quark masses rather than hadron masses. (See, however the discussion above on other phenomenological models.)

These considerations should be valid both for mesons and for baryons, with the additional complication of diquark production for the latter. While for strangeness suppression for mesons one parameter is needed, an additional suppression for diquark (su and sd) production is introduced. No further parameter controls the ss-diquark production. The too high $\Omega^{-}$production at PEP/PETRA (see Section 5.3 and Figure 5.3), which now at LEP agrees between data and JETSET, is a great success of the concept of tunneling.

Vector mesons are particularly suited for this study. They are abundant enough to allow an accurate study, and unlike pseudoscalar mesons, rarely originate from decays. Hence, a first guess on the strangeness suppression $s / u$ is obtained directly from the particles without any (model dependent) correction.

We make the assumption that the relative production of non-strange and strange particles is governed by the frequency by which an up or down quark is replaced by a strange quark, the up and down quark being equally frequently produced. In Figure 7.5 ratios representing $N(s) / N(u)$ (usually abbreviated $s / u$ ) calculated from the average particle multiplicities (see Table 5.2), are displayed. A comparison with the JETSET and HERWIG models are given.

These uncorrected values are in good agreement with the prediction of the models for the pseudoscalars, $s / u=\frac{1}{4}\left(\mathrm{~K}^{0}+\mathrm{K}^{ \pm}\right) / \frac{1}{3}\left(\pi^{0}+\pi^{ \pm}\right)$, and for the vector mesons, $\frac{1}{4}\left(\mathrm{~K}^{* \pm}+\right.$ $\left.\mathrm{K}^{* 0}\right) / \frac{1}{2}\left(\rho^{0}+\omega\right), \phi / \frac{1}{4}\left(\mathrm{~K}^{* \pm}+\mathrm{K}^{* 0}\right)$. For the spin- $1 / 2$ baryon octet, $\Lambda / \mathrm{p}, \frac{1}{3}\left(\Sigma^{0}+\Sigma^{ \pm}\right) / \mathrm{p}$, $\Xi^{-} / \frac{1}{3}\left(\Sigma^{0}+\Sigma^{ \pm}\right), \Xi^{-} / \Lambda$, the ratios including the $\Xi^{-}$are not well reproduced by the models (The HERWIG prediction for $\Xi^{-} / \frac{1}{3}\left(\Sigma^{0}+\Sigma^{ \pm}\right)$is off scale in Figure 7.5). In the measurement with the spin-3/2 decuplet baryons, $\frac{1}{2} \Sigma^{* \pm} / \Delta^{++}, \Xi^{* 0} / \frac{1}{2} \Sigma^{* \pm}, \Omega^{-} / \Xi^{* 0}$, some deviations are seen, the uncertainties on the data do not allow for definitive conclusions. The ratio for tensor mesons $\sqrt{f_{2}^{\prime} / f_{2}}$ is in good agreement.

The strangeness suppression differs from the naive expectation of 0.3 in most of the ratios: most of the light hadrons originate from decays. In Table 7.2, we see that only $13 \%$ of the pions do not originate from decays; $42 \%$ for proton and $33 \%$ for Lambda, the fractions for other light hadrons are listed in Table 7.2. The effect on the strangeness suppression is shown in Figure 7.5, where $s / u$ increases from 0.12 to 0.30 , when particle decays in JETSET are switched off. Furthermore one may exclude particles which contain quarks from the parton shower: this removes the leading particles. The $s / u$ values are now closer to the expected 0.3 .

For the data, we do not have the information whether a particle comes from a decay or from the string. However, we can correct for decays of identified particles, maybe for mesons (pseudoscalar and vector mesons), baryons or both. E.g., for the $\mathrm{K}^{ \pm}$, we correct for decays of $\mathrm{K}^{* \pm}, \mathrm{K}^{* 0}, \phi$, and $\Omega^{-}$. The estimate for $s / u$ is now closer to 0.3 than without 


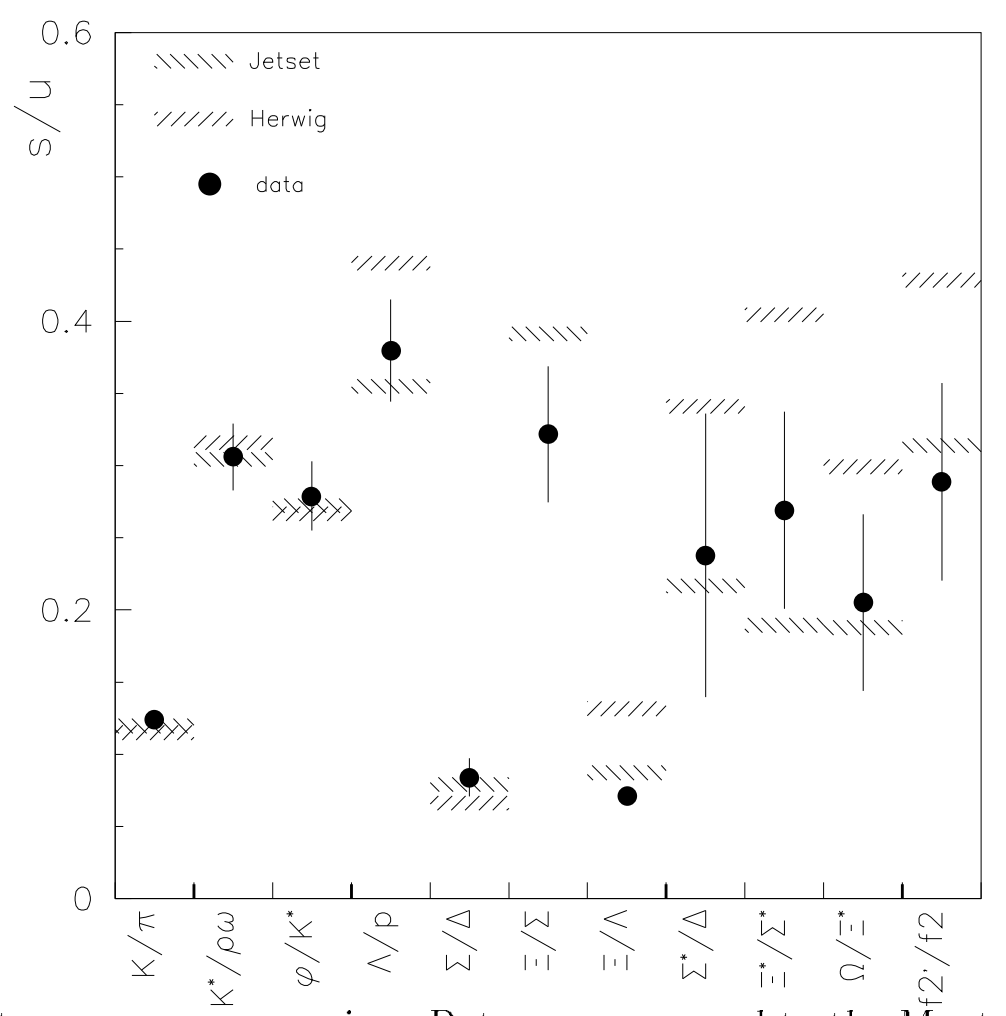

Figure 7.5: Strangeness suppression: Data are compared to the Monte Carlo predictions.

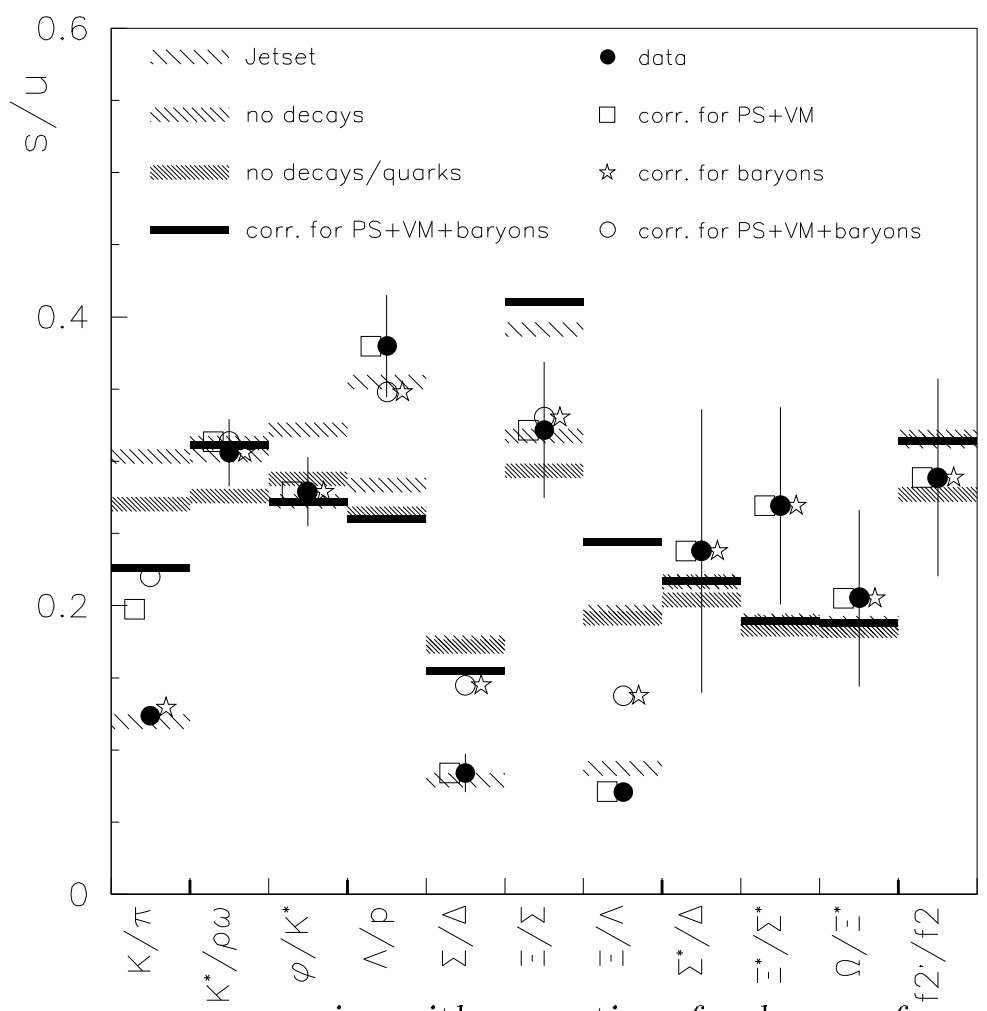

Figure 7.6: Strangeness suppression with corrections for decays of pseudoscalar and vector mesons (PS, VM) and baryons. For the Jetset model ratios are also shown excluding decay products and those containing quarks from the parton shower, which removes hadrons containing a leading quark. 


\begin{tabular}{|l|l|l|l|}
\hline Particle & JETSET 7.4 & no decay [\%] & no decay/no quark [\%] \\
\hline all charged & 20.59 & 16 & 15 \\
\hline$\pi^{ \pm}$ & 16.86 & 13 & 12 \\
$\pi^{0}$ & 9.65 & 12 & 11 \\
$\mathrm{~K}^{ \pm}$ & 2.14 & 31 & 26 \\
$\mathrm{~K}^{0}$ & 2.083 & 32 & 26 \\
$\eta$ & 1.062 & 54 & 49 \\
$\eta^{\prime}$ & 0.155 & 82 & 73 \\
\hline$\rho^{0}$ & 1.286 & 57 & 52 \\
$\mathrm{~K}^{* \pm}$ & 0.774 & 59 & 48 \\
$\mathrm{~K}^{* 0}$ & 0.777 & 59 & 48 \\
$\omega$ & 1.261 & 58 & 53 \\
$\phi$ & 0.105 & 70 & 51 \\
\hline $\mathrm{f}_{0}$ & 0.046 & 93 & 84 \\
$\mathrm{f}_{2}$ & 0.209 & 96 & 86 \\
$\mathrm{f}_{2}^{\prime}$ & 0.021 & 98 & 67 \\
$\mathrm{~K}_{2}^{* 0}$ & 0.127 & 98 & 76 \\
\hline $\mathrm{p}$ & 1.068 & 42 & 40 \\
$\Delta^{++}$ & 0.159 & 97 & 94 \\
$\Lambda$ & 0.379 & 33 & 30 \\
$\Sigma^{0}$ & 0.087 & 91 & 87 \\
$\Sigma^{ \pm}$ & 0.167 & 92 & 88 \\
$\Xi^{-}$ & 0.033 & 74 & 66 \\
$\Sigma^{* \pm}$ & 0.086 & 97 & 89 \\
$\Xi^{0 *}$ & 0.0065 & 96 & 86 \\
$\Omega^{-}$ & 0.0012 & 95 & 83 \\
\hline
\end{tabular}

Table 7.2: Total multiplicity of light flavoured hadrons predicted with the JETSET model. The third column gives the fraction of hadrons produced, when particle decays are switched off; in the fourth column, hadrons containing quarks from the parton shower are excluded, too.

correction. Proceeding in the same way with the JETSET model shows the quality of the model (see Figure 7.6).

One should certainly consider scalar and tensor meson production. The measurements, however, are still too poor to include them in the aforementioned procedure. The comparison between data and model predictions after correction for decays of light flavours only, implies that the production of heavy flavours and their branching ratios are sufficiently well known.

Another approach for the $s / u$ determination, which avoids the complication of correcting for decays, was summarized recently in Reference [3] for LEP data. Especially interesting is the ratio of charged pions and kaons measured at high momenta [12, 13, 128$]$. The ratio of strange heavy flavour mesons to non-strange ones [39, 40, 53, 54, 131] is well suited for a determination of $s / u$ : b-hadrons (c-hadrons) may only come from b-hadrons (b- or c-hadrons), rather than from many decays like light flavoured hadrons. The L3 
Collaboration [132] has used the fact that B-hadrons mix and that the mixing parameter depends on the relative flavour composition of the data set. All these measurements agree on a strangeness suppression with a factor around 0.3 .

\section{c) Angular Momentum Suppression}

The greater mass of diquarks and strange quarks motivates the lower production cross section of baryons and the suppression of strange hadrons. The available phase space for particles with spin is larger. Again (as for $s / u$ ), vector and pseudoscalar mesons provide valuable information about the relative probabilities for the corresponding spin states to be produced in the hadronization already without any correction.

In JETSET the probability to produce mesons with spin 1 is controlled by the additional parameters (see Table 3.3), e.g., for light flavours $[\mathrm{V} /(\mathrm{V}+\mathrm{P})]_{\mathrm{u}, \mathrm{d}}$ and $[\mathrm{V} /(\mathrm{V}+\mathrm{P})]_{\mathrm{s}}$, with default values of 0.5 and 0.6 , which are lower than the expectation from spin state counting. This ratio pertains to mesons directly produced in the hadronization.

It is worth noting that mesons with orbital angular momentum are suppressed even more. Better measurements of their production are needed, however, for an improved description of inclusive spectra.

For baryons the spin-3/2 decuplet can be compared with the spin-1/2 octet. The comparison has to cope with a large fraction of baryons being produced from decays for some and a low production rate for the other baryons.

The data and calculations for spin suppression, $[\mathrm{V} /(\mathrm{V}+\mathrm{P})]$ for mesons, $\frac{1}{2}\left(\rho^{0}+\omega\right) /\left(\frac{1}{2}\left(\rho^{0}+\right.\right.$ $\left.\omega)+\frac{1}{3}\left(\pi^{0}+\pi^{ \pm}\right)\right),\left(\mathrm{K}^{* 0}+\mathrm{K}^{* \pm}\right) /\left(\left(\mathrm{K}^{* 0}+\mathrm{K}^{* \pm}\right)+\left(\mathrm{K}^{0}+\mathrm{K}^{ \pm}\right)\right)$, or spin- $\frac{3}{2} /\left(\operatorname{spin}-\frac{3}{2}+\operatorname{spin}-\frac{1}{2}\right)$ for baryons, $\Delta^{++} /\left(\Delta^{++}+p\right), \frac{1}{2} \Sigma^{* \pm} /\left(\frac{1}{2} \Sigma^{* \pm}+\Lambda\right), \frac{1}{2} \Sigma^{* \pm} /\left(\frac{1}{2} \Sigma^{* \pm}+\frac{1}{3}\left(\Sigma^{0}+\Sigma^{*}\right)\right), \Xi^{* 0} /\left(\Xi^{* 0}+\Xi^{-}\right)$, are shown in Figures 7.7 and 7.8 ; with the same procedures for the correction applied as for the strangeness suppression. The JETSET model is closer to the data than the Herwig model. However, the input value for $[\mathrm{V} /(\mathrm{V}+\mathrm{P})]_{\mathrm{u}, \mathrm{d}, \mathrm{s}}=0.51$ is not reached after corrections for particle decays.

\section{d) Dependence on Hadron Mass}

The modified leading logarithmic approximations (MLLA) combined with local parton hadron duality (LPHD) predicts that the position of the maximum of the $\xi_{p}=\ln \left(1 / x_{p}\right)$ distribution should decrease with increasing hadron mass. In Table 7.3 the maximum positions $\xi^{*}$ for the various particles are listed. (Recent reviews may be found in Ref. $[36,64]$.) Most values have been taken from the original papers; when not given, the values were taken from Ref. [64]. Figure 7.9a shows for each particle the value of $\xi^{*}$ obtained by averaging all experimental values. As already pointed out by DELPHI [36] and is easily seen in the figure, the data are not described by one exponential line, but rather by two; one for mesons and another one for baryons. It may be suspected that pseudoscalar and vector mesons lie on two different lines.

In Ref. [36] it is argued that a better description is obtained, when the measurements are corrected for decays. In Table 7.3 we give the shift of the maximum position as determined with JETSET. The shift is obtained as the difference of tuned JETSET with particle decays enabled and disabled. In Figure $7.9 \mathrm{~b}$ the corrected maxima are shown as 


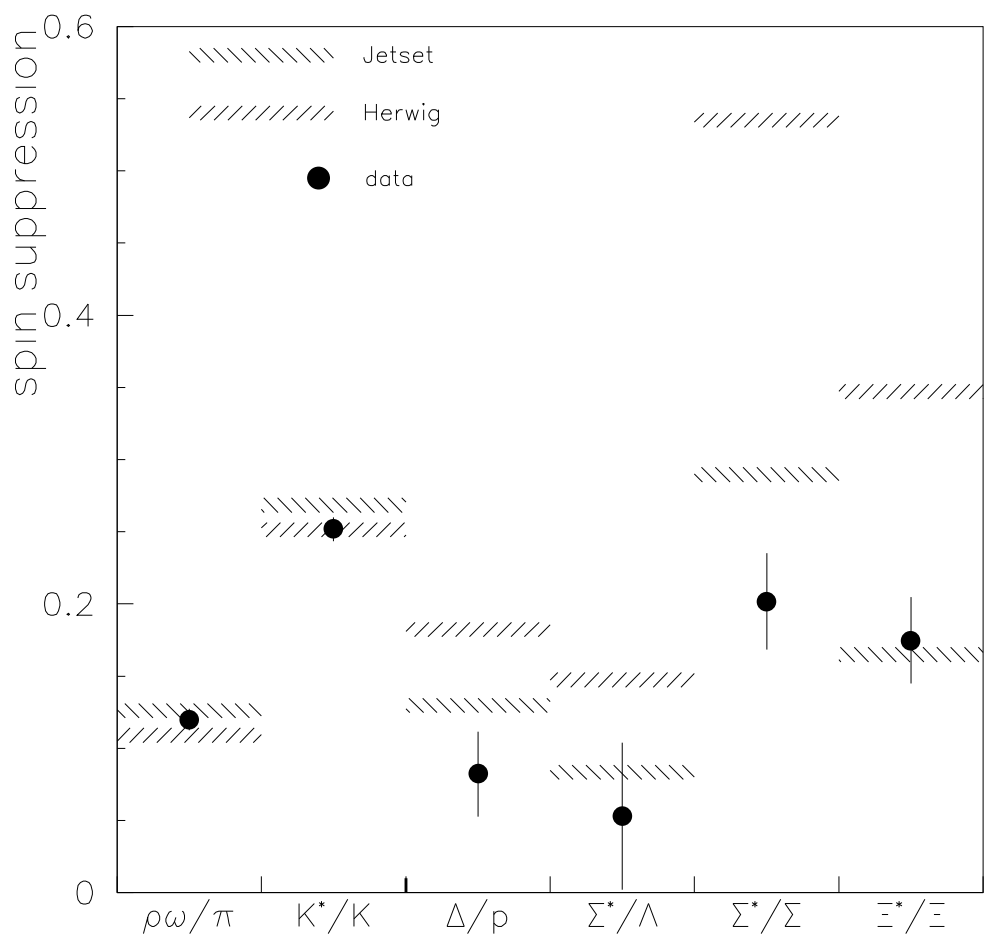

Figure 7.7: Spin suppression: Data are compared to the Monte Carlo predictions. Further details may be found in the text.

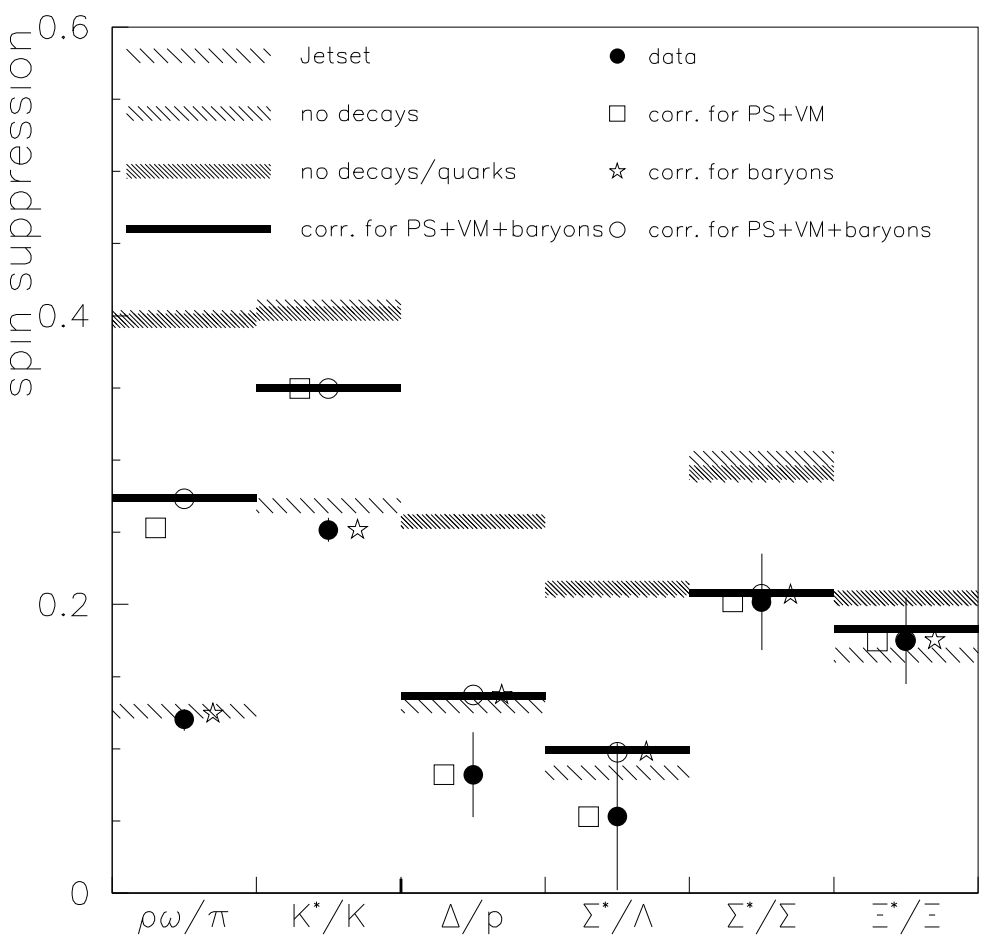

Figure 7.8: Spin suppression with corrections for decays of pseudoscalar and vector mesons. For the Jetset model ratios are also shown excluding decay products and those containing quarks from the shower. 


\begin{tabular}{|c|c|c|c|c|}
\hline Particle & Mass $/ \mathrm{GeV} / c^{2}$ & $\xi^{*}$ & $\Delta \xi^{*}$ & Exp. \\
\hline$\pi^{0}$ & 0.135 & $3.96 \pm 0.13$ & -0.13 & L3 \\
\hline$\pi^{0}$ & 0.135 & $\begin{array}{l}3.90_{-0.14}^{+0.24} \\
3.94 \pm 0.11\end{array}$ & -0.13 & $\begin{array}{l}\text { DELPHI } \\
\text { average }\end{array}$ \\
\hline$\pi^{ \pm}$ & 0.140 & $3.78 \pm 0.02$ & -0.15 & ALEPH \\
\hline$\pi^{ \pm}$ & 0.140 & $\begin{array}{l}3.81 \pm 0.02 \\
3.80 \pm 0.01\end{array}$ & -0.15 & $\begin{array}{l}\text { OPAL } \\
\text { average }\end{array}$ \\
\hline all charged & 0.22 & $3.618 \pm 0.028$ & -0.21 & ALEPH \\
\hline all charged & 0.22 & $3.67 \pm 0.10$ & -0.21 & DELPHI \\
\hline all charged & 0.22 & $3.71 \pm 0.05$ & -0.21 & L3 \\
\hline all charged & 0.22 & $\begin{array}{l}3.603 \pm 0.042 \\
3.63 \pm 0.02\end{array}$ & -0.21 & $\begin{array}{l}\text { OPAL } \\
\text { average }\end{array}$ \\
\hline $\mathrm{K}^{ \pm}$ & 0.494 & $2.70 \pm 0.09$ & 0.68 & ALEPH \\
\hline $\mathrm{K}^{ \pm}$ & 0.494 & $2.63 \pm 0.07$ & 0.68 & DELPHI \\
\hline $\mathrm{K}^{ \pm}$ & 0.494 & $\begin{array}{l}2.63 \pm 0.04 \\
2.64 \pm 0.03\end{array}$ & 0.68 & $\begin{array}{l}\text { OPAL } \\
\text { average }\end{array}$ \\
\hline $\mathrm{K}^{0}$ & 0.498 & $2.63 \pm 0.04$ & 0.62 & ALEPH \\
\hline $\mathrm{K}^{0}$ & 0.498 & $2.62 \pm 0.11$ & 0.62 & DELPHI \\
\hline $\mathrm{K}^{0}$ & 0.498 & $2.89 \pm 0.05$ & 0.62 & L3 \\
\hline $\mathrm{K}^{0}$ & 0.498 & $\begin{array}{l}2.91 \pm 0.04 \\
2.79 \pm 0.08\end{array}$ & 0.62 & $\begin{array}{l}\text { OPAL } \\
\text { average }\end{array}$ \\
\hline$\eta$ & 0.547 & $2.52 \pm 0.10$ & 0.22 & $\begin{array}{l}\mathrm{L} 3 \\
\text { average }\end{array}$ \\
\hline$\rho^{0}$ & 0.770 & $2.80 \pm 0.19$ & 0.54 & $\begin{array}{l}\text { ALEPH } \\
\text { average }\end{array}$ \\
\hline$\omega$ & 0.782 & $2.80 \pm 0.36$ & 0.17 & $\begin{array}{l}\mathrm{L} 3 \\
\text { average }\end{array}$ \\
\hline$K^{* 0}$ & 0.896 & $2.26 \pm 0.05$ & 0.71 & ALEPH \\
\hline$K^{* 0}$ & 0.896 & $\begin{array}{l}2.40 \pm 0.04 \\
2.35 \pm 0.07\end{array}$ & 0.71 & $\begin{array}{l}\text { OPAL } \\
\text { average }\end{array}$ \\
\hline $\mathrm{p}$ & 0.938 & $2.85 \pm 0.18$ & -0.02 & ALEPH \\
\hline $\mathrm{p}$ & 0.938 & $2.96 \pm 0.16$ & -0.02 & DELPHI \\
\hline $\mathrm{p}$ & 0.938 & $\begin{array}{l}3.00 \pm 0.09 \\
2.97 \pm 0.07\end{array}$ & -0.02 & $\begin{array}{l}\text { OPAL } \\
\text { average }\end{array}$ \\
\hline$\eta^{\prime}$ & 0.958 & $2.47 \pm 0.49$ & 0.53 & $\begin{array}{l}\text { L3 } \\
\text { average }\end{array}$ \\
\hline$\phi$ & 1.019 & $2.21 \pm 0.03$ & 0.97 & ALEPH \\
\hline$\phi$ & 1.019 & $2.11 \pm 0.06$ & 0.97 & DELPHI \\
\hline$\phi$ & 1.019 & $\begin{array}{l}2.29 \pm 0.05 \\
2.21 \pm 0.04\end{array}$ & 0.97 & $\begin{array}{l}\text { OPAL } \\
\text { average }\end{array}$ \\
\hline$\Lambda$ & 1.116 & $2.67 \pm 0.14$ & 0.03 & ALEPH \\
\hline$\Lambda$ & 1.116 & $2.82 \pm 0.25$ & 0.03 & DELPHI \\
\hline$\Lambda$ & 1.116 & $2.83 \pm 0.13$ & 0.03 & L3 \\
\hline$\Lambda$ & 1.116 & $\begin{array}{l}2.77 \pm 0.05 \\
2.77 \pm 0.04\end{array}$ & 0.03 & $\begin{array}{l}\text { OPAL } \\
\text { average }\end{array}$ \\
\hline$\Xi^{-}$ & 1.321 & $2.60 \pm 0.16$ & 0.06 & DELPHI \\
\hline$\Xi^{-}$ & 1.321 & $\begin{array}{l}2.57 \pm 0.11 \\
2.58 \pm 0.09\end{array}$ & 0.06 & $\begin{array}{l}\text { OPAL } \\
\text { average }\end{array}$ \\
\hline
\end{tabular}

Table 7.3: Maximum position $\xi^{*}$ of the $\xi=\ln \left(1 / x_{p}\right)$ distribution and its shift $\Delta \xi^{*}$ due to particle decays as computed with JETSET. For references see citations in the text and Table 5.1. The particle masses are taken from Ref. [79]; the mass for all charged is the average mass of $\pi^{ \pm}, \mathrm{K}^{ \pm}$and $\mathrm{p}$, weighted with their production probability. 

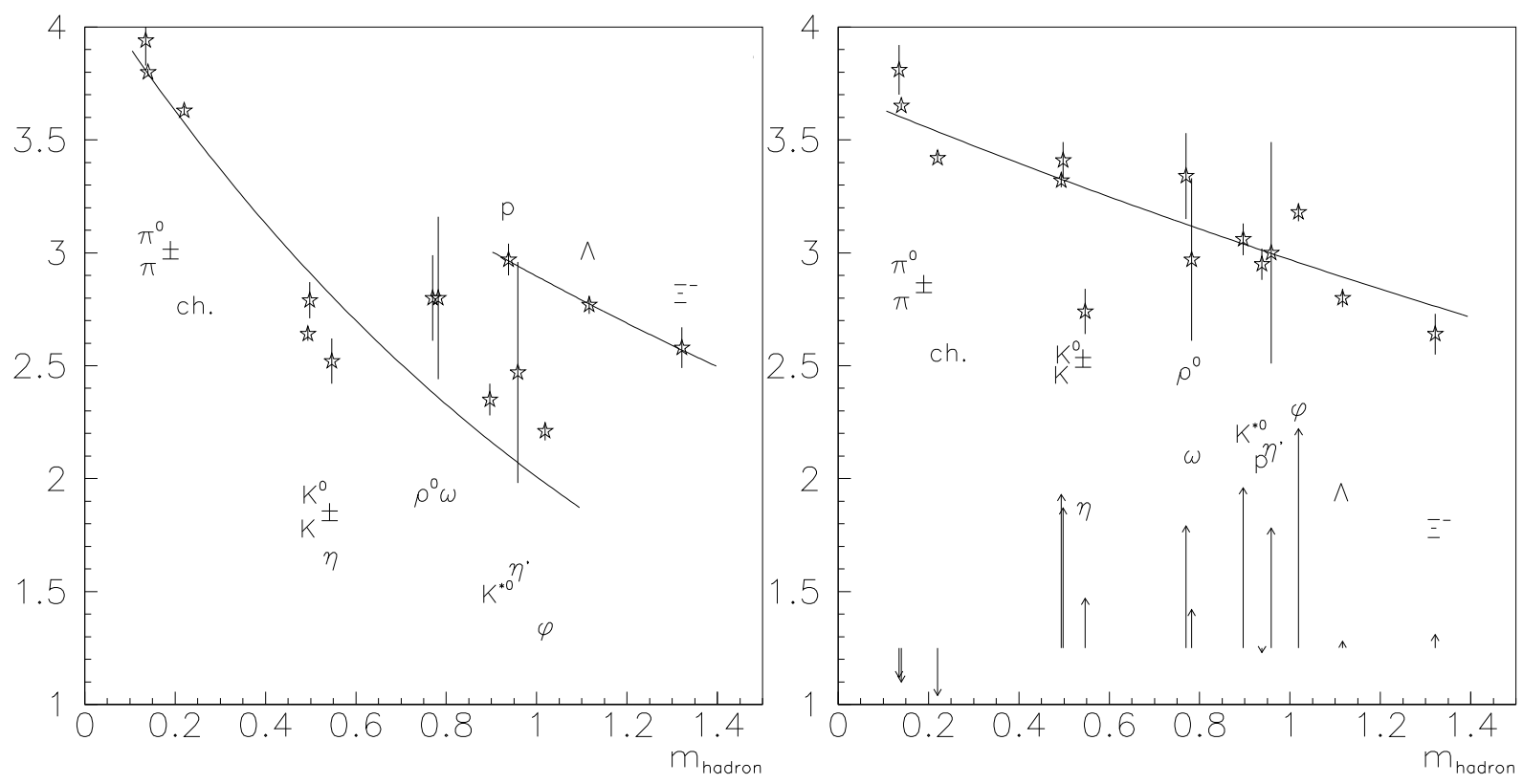

Figure 7.9: $\xi^{*}$-dependence on hadron mass. The first figure shows the data with a separate fit with an exponential function to mesons and baryons. In the second figure data are corrected for decays; the corrections, which were applied are indicated by the arrows.

function of the particle mass. The arrows indicate the corrections, that were applied. A fit with a single exponential function nicely describes the data.

This agreement after correction is not really a success of MLLA combined with LPHD. In this approach particle decays should be just another part of the fragmentation and therefore, no distinction should be made between these two aspects of hadronization in the LPHD approach [108].

\subsection{Dependence on Centre-of-Mass Energy}

In Section 5.3 we gave a brief overview of the data measured at lower centre-of-mass energy $[62,79,103,105,106]$.

The model tuning, which gives similar parameter values, with or without inclusion of lower energy data, implies that most aspects of particle production are understood and implemented in the models. More phenomenological ideas, based on thermal equilibrium or isospin etc., were compared to data at different centre-of-mass energies.

At the PETRA collider many aspects of the energy dependence have been studied. Some of these studies were repeated or improved at LEP. PETRA was a machine well suited for this, because $\sqrt{s}$ was varied from 7 to $44 \mathrm{GeV}$. The multiplicity distribution expressed in the variables $z=n_{c h} /<n_{c h}>$ and $\Phi(z)=<n_{c h}>\mathcal{P}\left(n_{c h}\right)$ is invariant with respect to energy, called KNO-scaling [133]. Measurements at LEP confirm the observation [8, 9, 11], Figure 7.10 shows the study done by the OPAL collaboration [11].

Here we give a few examples on aspects studied at LEP. Even without an energy scan, 


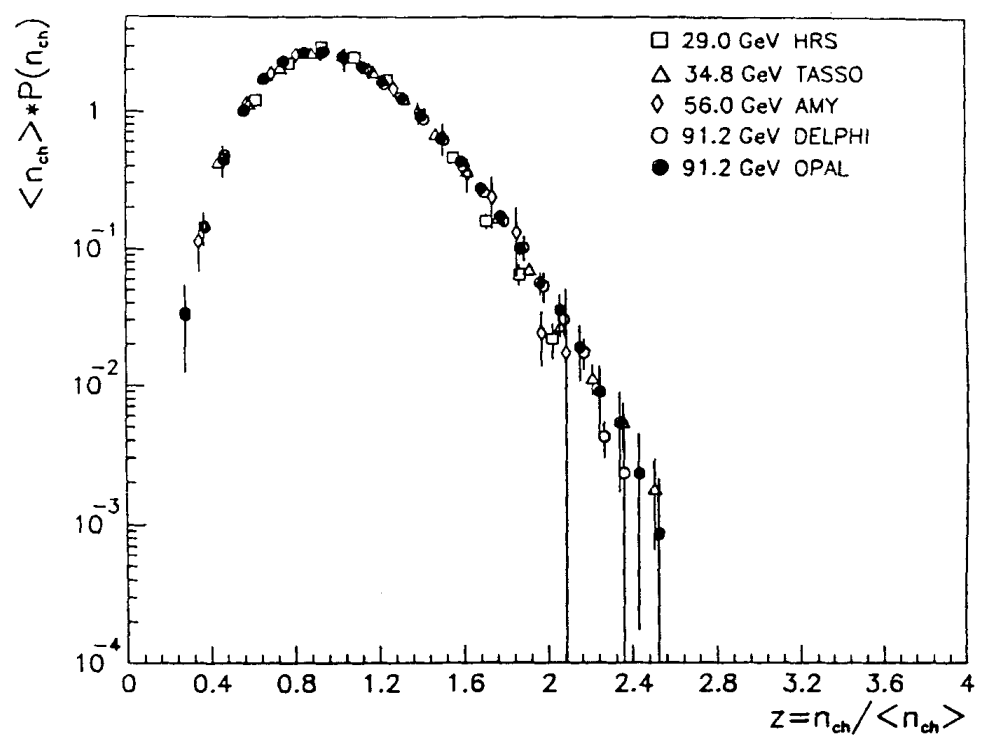

Figure 7.10: Multiplicity distribution as function of $z=n_{c h} /\left\langle n_{c h}\right\rangle$ for experiments at different centre-of-mass energies demonstrating the validity of KNO-scaling [11].

the DELPHI collaboration has studied the dependence using radiative events [134]. When a hard photon is emitted in the initial state, the effective energy of the remaining hadronic system in the final state is lower than the centre-of-mass energy. In Figure 7.11 the average charged particle multiplicity is shown. The solid line is a fit of the displayed data points to a parameterization, which was calculated including resummation of leading (LLA) and next-to-leading (NLLA) corrections [135], with an additional parameter allowing for higher order corrections in $\alpha_{\mathrm{s}}$. The dashed curve corresponds to a fit to $\mathrm{e}^{+} \mathrm{e}^{-}$data of different centre-of-mass energies published in Ref. [136], where it is claimed that $\mathrm{e}^{+} \mathrm{e}^{-}$ and $p \bar{p}$ data can be described with the same phenomenological parameterization.

The peak positions of the $\xi$ distribution have been measured by experiments at LEP and by colliders with lower centre-of-mass energies. The results for charged particles are collected in Table 7.4. In Figure 7.12 they are compared to the prediction of MLLA assuming LPHD and the incoherent parton shower without angular ordering. (The corresponding Table and Figure are taken from Ref. [64].) The data can be described by a single parameter $\Lambda_{\text {eff }}$. The slope obtained from the incoherent shower model is twice as high and ruled out. Precise measurements also exist for the stable particles, $\pi^{ \pm}, \mathrm{K}^{ \pm}$, $\mathrm{p}$ and a few neutral mesons. The energy dependence shown in Figure 7.13 [16] is nicely described by the MLLA prediction.

In Chapter 2 on theoretical aspects and the description of the models, we discussed that the fragmentation should be independent of the centre-of-mass energy. Therefore at PEP/PETRA energies many experiments used $(s / \beta) \mathrm{d} \sigma^{\mathrm{h}} / \mathrm{dx}$ E for discussing their inclusive spectra. Gluon emission and higher order QCD corrections lead to scaling violation. The 


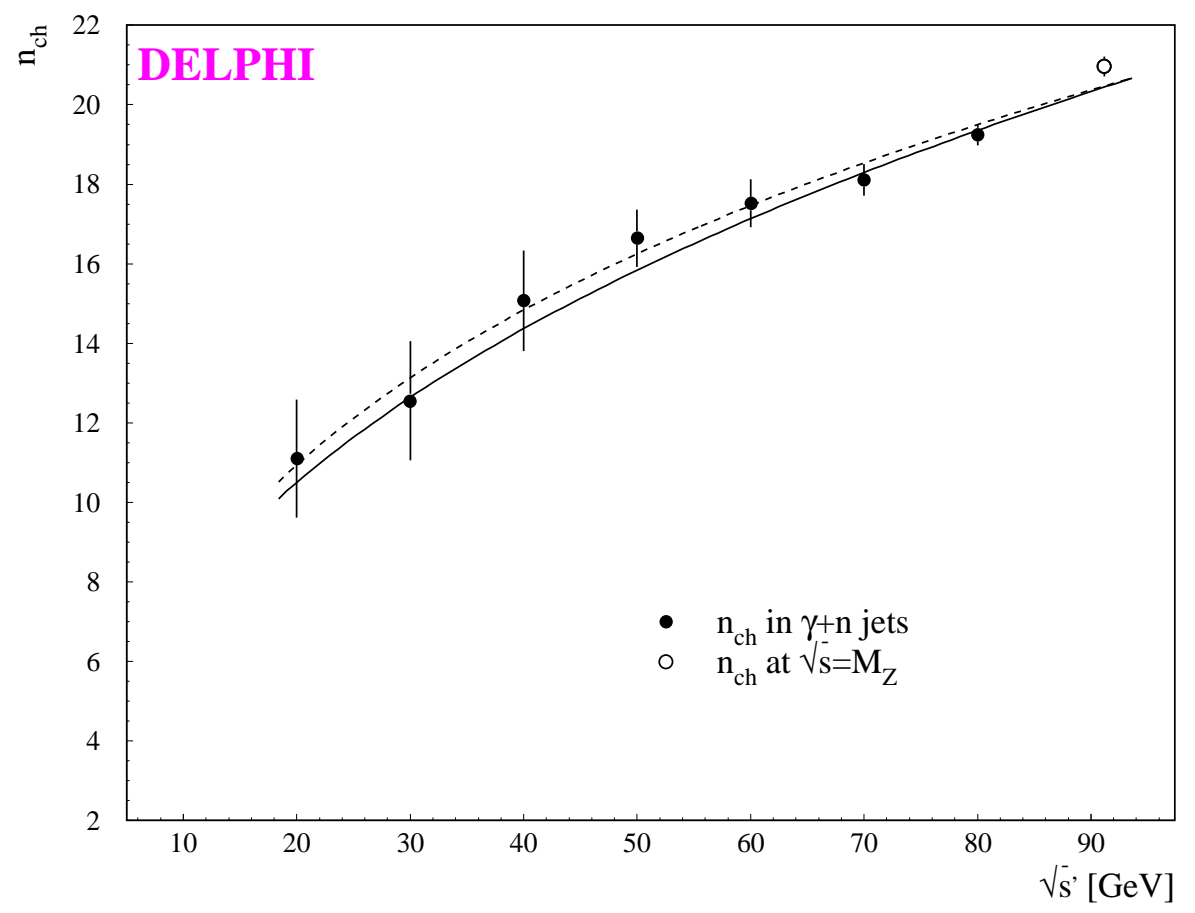

Figure 7.11: Charged multiplicity measured with the DELPHI detector as function of the effective centre-of-mass energy in $\mathrm{q} \overline{\mathrm{q}} \gamma$ events. The data are compared to a LLA motivated function (solid line) and a phenomenological parameterization (dashed line) [134].

\begin{tabular}{|l|l|l|}
\hline$\sqrt{s} \mathrm{GeV}$ & \multicolumn{1}{|c|}{$\xi^{*}$} & \multicolumn{1}{|c|}{ Exp. } \\
\hline 14. & $2.353 \pm 0.043$ & TASSO \\
22. & $2.651 \pm 0.041$ & TASSO \\
29. & $2.771 \pm 0.015$ & MARK II \\
29. & $2.866 \pm 0.060$ & TPC $/ 2 \gamma$ \\
35. & $3.063 \pm 0.024$ & TASSO \\
35. & $2.929 \pm 0.072$ & CELLO \\
44. & $3.120 \pm 0.054$ & TASSO \\
55. & $3.147 \pm 0.093$ & AMY \\
91.2 & $3.618 \pm 0.028$ & ALEPH \\
91.2 & $3.67 \pm 0.10$ & DELPHI \\
91.2 & $3.71 \pm 0.05$ & L3 \\
91.2 & $3.603 \pm 0.042$ & OPAL \\
\hline
\end{tabular}

Table 7.4: Maximum position $\xi^{*}$ for charged particles as function of the centre-of-mass energy [64]. 


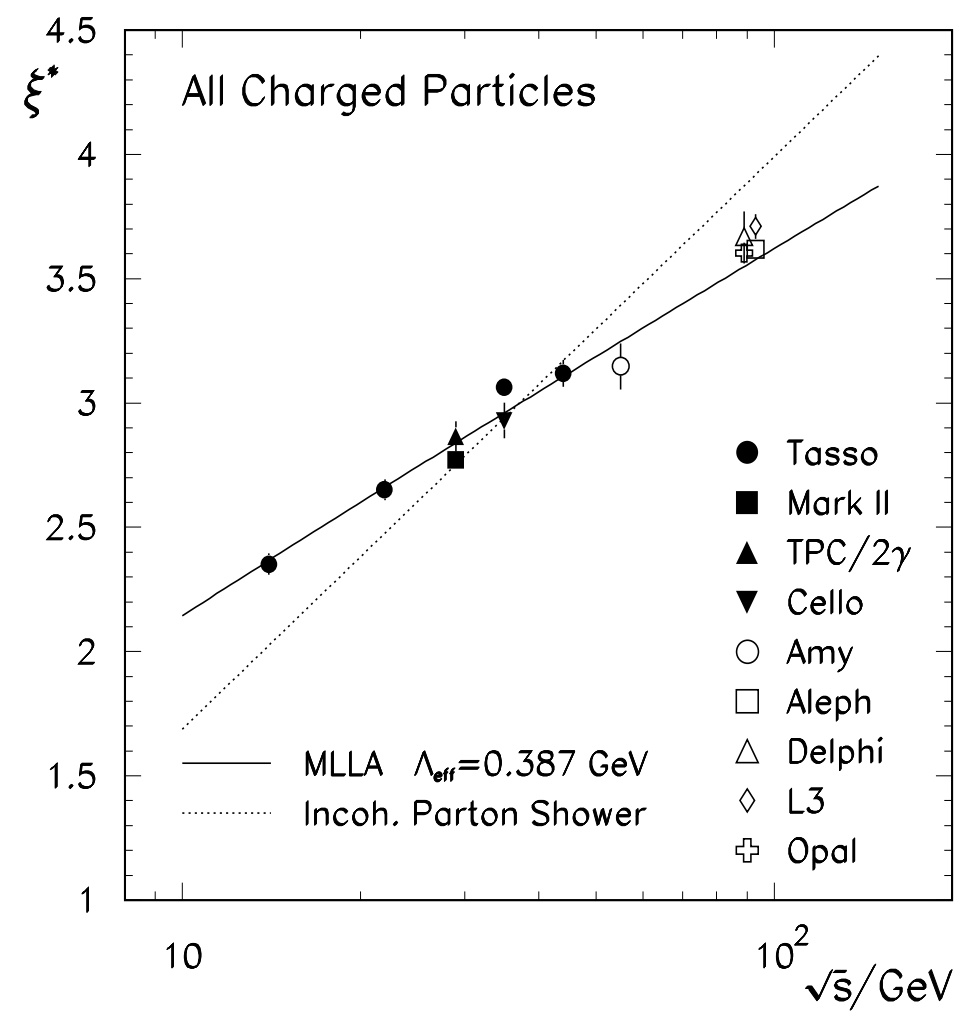

Figure 7.12: Peak position $\xi^{*}$ for charged particles as function of centre-of-mass energy. The full line is a fit to the MLLA prediction; the dotted line is the expectation for an incoherent parton shower [64].

ALEPH and DELPHI collaboration used this effect for measuring the strong coupling constant $[8,9]$ to $0.126 \pm 0.009$ and $0.118 \pm 0.005$, which is in agreement with other measurements of $\alpha_{\mathrm{s}}$.

In Figure 7.14, the scaled-energy distributions $\left(x_{E}\right)$ for experiments from $\sqrt{s}=22 \mathrm{GeV}$ to $91.2 \mathrm{GeV}$ are fitted to functions from next-to-leading calculations. The prediction from QCD for these all-flavour samples is in good agreement with the data. The scaling violation is more clearly demonstrated in Figure 7.15, where the ratio of the ALEPH and TASSO data in $x_{E}$ is compared to the fit result. The expectation with the flavour composition kept constant at the value at $91.2 \mathrm{GeV}$ is shown as dashed line. If no scaling violation was observed, the ratio should be unity for all $x_{E}$. In both figures, the dots are data used for the fit; the open circles are not used.

\subsection{Particle Composition in Flavour Tagged Events}

The difference of jets with different flavours allows for test of perturbative QCD. Several predictions on the different particle multiplicity of heavy and light flavour initiated jets exist [137] and comparison to data at different centre-of-mass energies have been done 


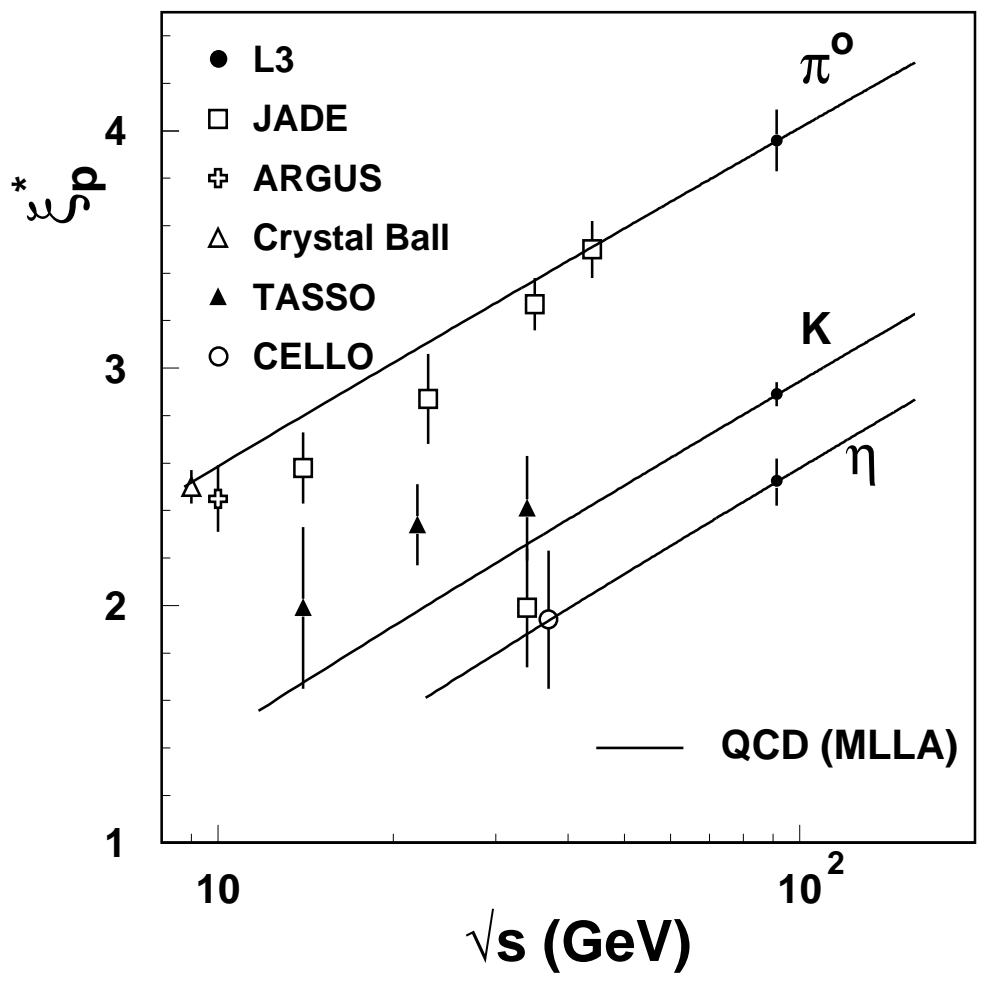

Figure 7.13: Peak position $\xi^{*}$ as function of centre-of-mass energy for neutral mesons together with a fit to the MLLA prediction [16].

(See Ref. [138, 139] for a recent review.). Calculations for heavy flavours are possible in perturbative QCD, because the quark mass is larger than the scale $\Lambda_{Q C D}$ and smaller than the centre-of-mass energy. The heavy quark mass leads to a natural cutoff, avoiding the non-perturbative domain of strong interaction. In the forward direction, where $\theta<$ $m_{Q} / E_{Q}$, known as 'dead cone', the particle yield is suppressed. This suppression in particle multiplicity is thought to be independent of $E_{c m s}$. The loss may be estimated assuming that the multiplicity of the heavy quarks, without the particles from the decay of the heavy hadron itself, corresponds to the multiplicity of light quarks at reduced cmsenergy. Furthermore the decay products, including weak decays of the B-meson must be included. The prediction for the difference of multiplicity in b- and c-jets with respect to light flavour initiated jets are $\delta_{c l}=1.7 \pm 0.5$ and $\delta_{b l}=5.5 \pm 0.8$. Modification of these calculations gave upper bounds $\delta_{c l}<1.3$ to 1.7 and $\delta_{c l}<3.7$ to 4.1. Assuming masses for the heavy quarks $m_{c}=1.5 \mathrm{GeV} / c^{2}$ and $m_{b}=4.8 \mathrm{GeV} / c^{2}$ gives predictions of $\delta_{c l}=1.01$ and $\delta_{b l}=3.68$.

A more naïve model assumes that the non-leading energy, i.e., the energy without that of the leading quark, has the multiplicity of a light flavoured quark with this lower energy. After correction for the decay products of the heavy quark, a naive model prediction is obtained. The parameterization depends on the assumption made for the particle 


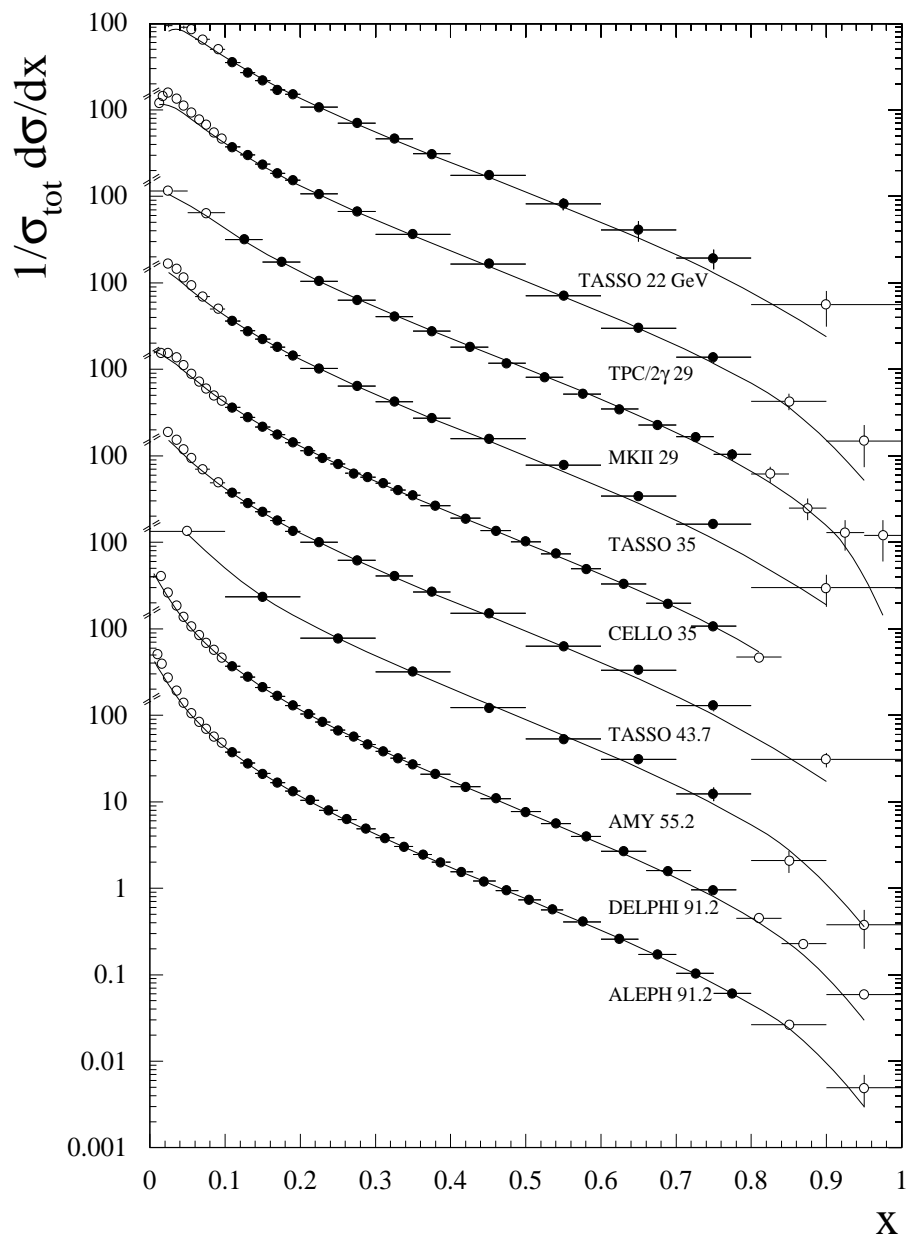

Figure 7.14: Inclusive charged particles energy distribution at various centre-of-mass energies with the fit to $Q C D$-prediction showing scaling violation. The curves are the result of the fit. Data points used are shown as dots [8].

multiplicity as function of $E_{c m s}$, the heavy flavour decay and its fragmentation function $[140,141]$.

From an experimental point of view, one must distinguish b- and c- jets from light flavour jets. B-hadrons have short lifetimes and their decay lengths are several $100 \mu \mathrm{m}$. The lifetime-tag relies on this together with the excellent resolution $(\sigma \approx 20 \mu \mathrm{m})$ of the impact parameter $d_{i}$, which gives the closest approach to the beam line. The sign is positive if the track intersects the B-hadron direction, which is approximated by the jetor thrust direction. From tracks in one hemisphere, as defined by the thrust axis, and their signed impact parameter significance $S=d_{i} / \sigma_{i}$ the b-jet probability is calculated; the impact parameter significance is obtained with the data from tracks having a negative impact parameter, i.e., no lifetime. The event is divided in two hemispheres in order to avoid biases due to the tagging procedure: one side is used for tagging, the other is studied. 


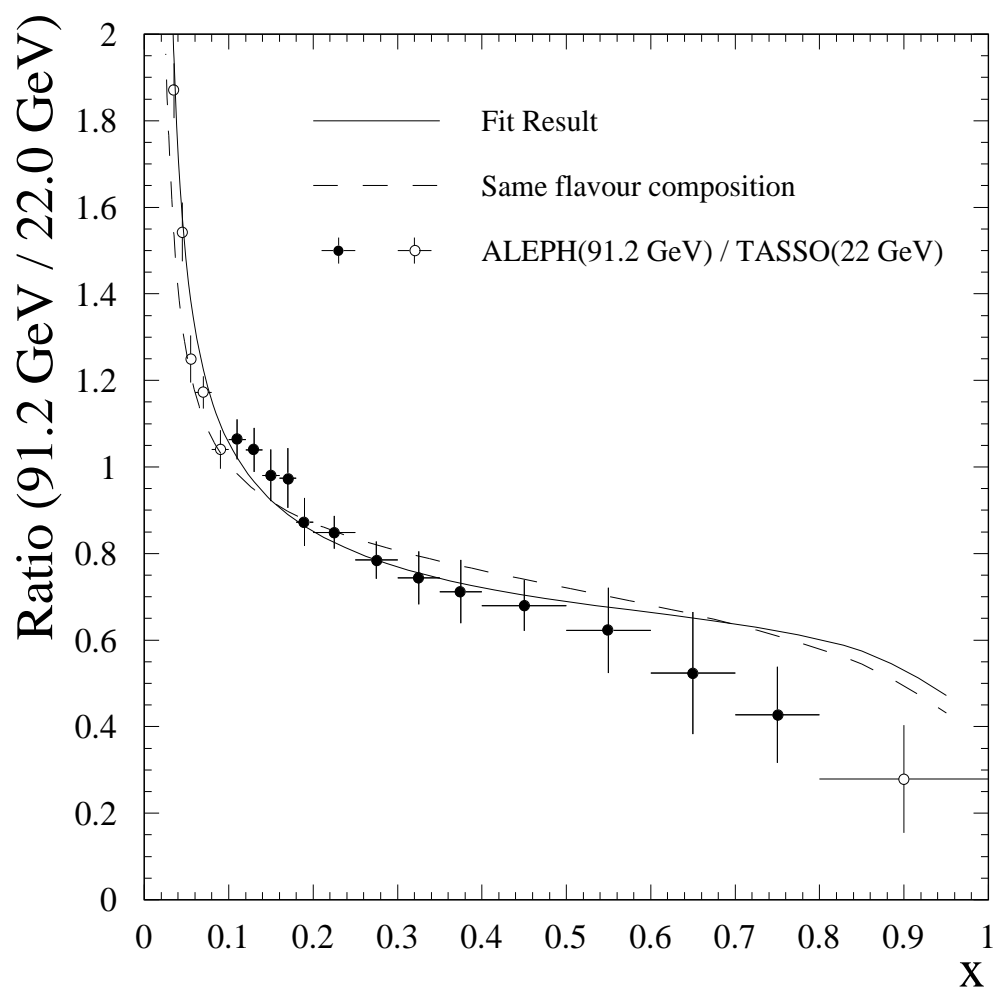

Figure 7.15: Ratio of inclusive energy distribution at $\sqrt{s}=91.2 \mathrm{GeV}$ and $22 \mathrm{GeV}$ compared to the fit to QCD-prediction demonstrating scaling violation (solid curve). The expectation for the same flavour composition is shown as dashed curve [8].

This procedure is used by all LEP experiments [140, 142, 143]. Another possibility for btagging uses the fact that the mass of the b-quark is much higher than those of light quarks. B-hadrons, decaying semi-leptonically, emit leptons with high transverse momentum with respect to the jet axis.

The OPAL collaboration has studied particle multiplicity in c-events, using $\mathrm{D}^{*}$ mesons for their tag. Selecting $D^{*}$ mesons with different momenta gives samples of different $c$ and b- purity. This allows a simultaneous determination of the multiplicity in b-, c-, and uds- events [141].

The theoretical predictions as a function of $E_{c m s}$, together with experimental results are given in Fig. 7.16. The measurements at LEP are compiled in Table 7.5 [139, 140, 141].

The production of identified particles in flavour tagged events has been studied by the DELPHI collaboration $[140,144,145]$ with $\pi^{0}, \mathrm{~K}^{+}, \mathrm{K}^{0}$, and $\phi$ mesons and with protons, and $\Lambda$ baryons. The extraction of the signal proceeds as for the inclusive production with one difference - events have to be classified to originate from a primary b $\bar{b}$ quark pair. These events are tagged by the particle lifetime in one hemisphere, while the inclusive particle production is studied in the opposite hemisphere as defined by the thrust axis. This reduces the bias of the particle multiplicity by the tag to a negligible level. The rates extracted are given in Table 7.6 together with the number coming from the decay of a B-hadron. For the latter, the rapidity distribution $\left(y=\frac{1}{2} \log \frac{E+p_{l}}{E-p_{l}}\right)$ of the particles 

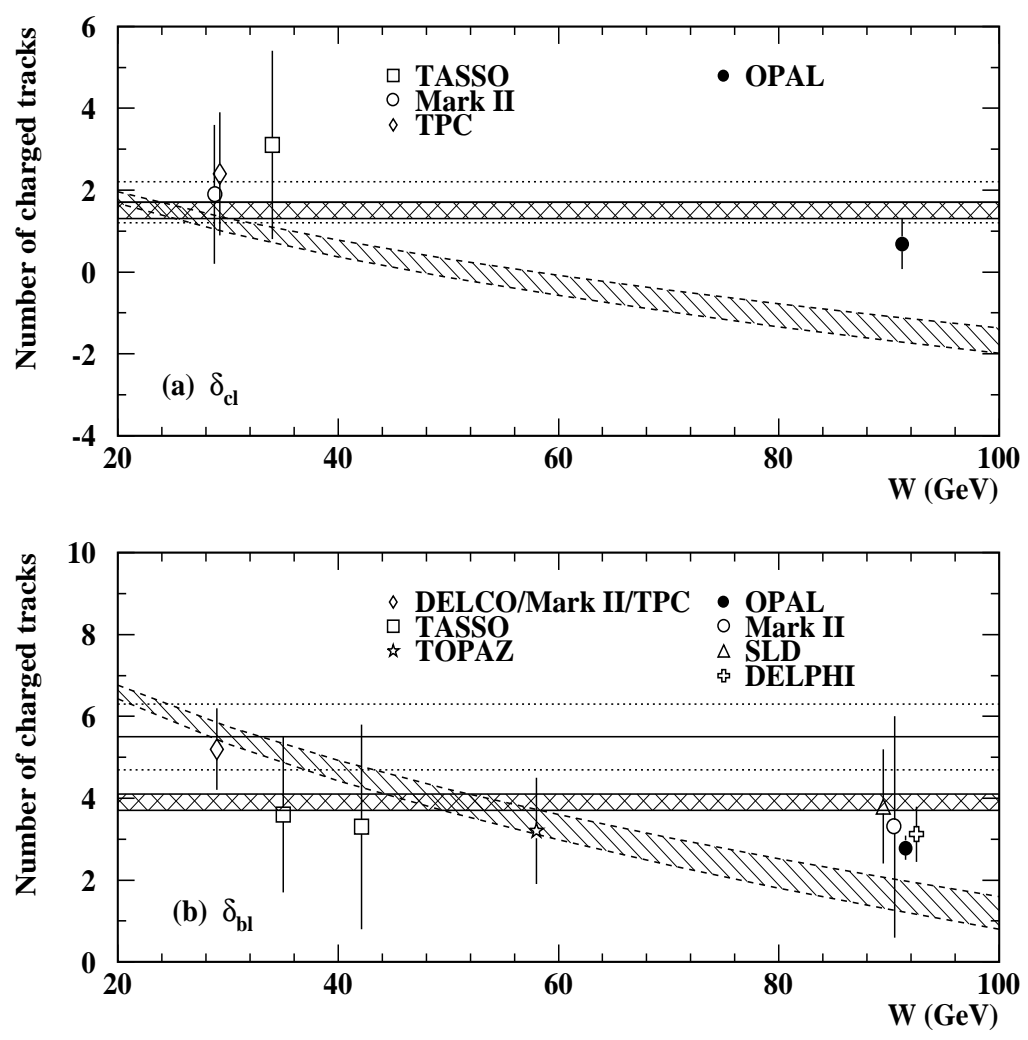

Figure 7.16: Difference of the charged multiplicity of $c$ - and b-flavoured quark jets to light flavoured quark jets in comparison with theoretical predictions: The single hatched areas are the prediction of the naive model. The cross hatched areas are upper limits from QCD. The MLLA prediction with its errors is indicated by the solid and dotted lines [141].

\begin{tabular}{|c|c|c|}
\hline & Value & Exp \\
\hline \hline $\bar{n}_{\mathrm{b} \overline{\mathrm{b}}}$ & $23.32 \pm 0.08 \pm 0.50$ & DELPHI \\
$\bar{n}_{\mathrm{b} \overline{\mathrm{b}}}$ & $23.62 \pm 0.02 \pm 0.48$ & OPAL \\
\hline $\bar{n}_{\mathrm{c} \overline{\mathrm{c}}}$ & $21.52 \pm 0.20 \pm 0.47$ & OPAL \\
\hline$\delta_{b l}$ & $3.12 \pm 0.09 \pm 0.67$ & DELPHI \\
$\delta_{b l}$ & $2.79 \pm 0.12 \pm 0.27$ & OPAL \\
\hline$\delta_{c l}$ & $0.69 \pm 0.51 \pm 0.35$ & OPAL \\
\hline
\end{tabular}

Table 7.5: Average charged multiplicity in $c \bar{c}$ and $\mathrm{b} \overline{\mathrm{b}}$ events, and the difference between $\mathrm{b} \overline{\mathrm{b}}(\mathrm{c} \overline{\mathrm{c}})$ and light quark events $\delta_{b l}\left(\delta_{c l}\right)[139,140,141]$. 
with respect to the thrust axis is fitted to a sum of two contributions: one distribution as expected from b-decay the other as expected from fragmentation; the shapes of the distributions were taken from JETSET Monte Carlo with Peterson fragmentation.

\begin{tabular}{|c|c|c|c|}
\hline Particle & Multiplicity (bb) & From B decay & Experiment \\
\hline \hline Charged & $23.43 \pm 0.48$ & $5.72 \pm 0.38$ & DELPHI,OPAL,SLD \\
\hline$\pi^{0}$ & $10.1 \pm 1.2$ & $2.78 \pm 0.53$ & DELPHI \\
\hline $\mathrm{K}^{ \pm}$ & $2.74 \pm 0.50$ & $0.88 \pm 0.19$ & DELPHI \\
\hline $\mathrm{K}^{0}$ & $2.16 \pm 0.12$ & $0.58 \pm 0.06$ & DELPHI \\
\hline$\phi$ & $0.126 \pm 0.023$ & $0.032 \pm 0.011$ & DELPHI \\
\hline $\mathrm{p}$ & $1.13 \pm 0.27$ & $0.141 \pm 0.059$ & DELPHI \\
\hline$\Lambda$ & $0.338 \pm 0.047$ & $0.059 \pm 0.011$ & DELPHI \\
\hline
\end{tabular}

Table 7.6: Hadron multiplicities measured in $\mathrm{b} \overline{\mathrm{b}}$ events and $B$ hadrons [139].

\subsection{Particle Composition in Quark and Gluon Jets}

Since the discovery of the gluon jets at PETRA, intensive investigations of three-jet events and the differences of quark and gluon jets have been performed at $\mathrm{e}^{+} \mathrm{e}^{-}$colliders. The different colour factors of quark and gluon led to the expectation that gluon jets should have a higher particle multiplicity as compared to quark jets. Initial studies showed an enhanced particle flow between quark and gluon jets as compared to the region opposite to the gluon direction, i.e. between the quark and anti-quark jet. Another way to look at this first evidence for the string effect was by studying the difference of $q \bar{q} g$ and $q \bar{q} \gamma$ events at the PETRA and PEP collider (see Figure 3.8 for a measurement at LEP).

At LEP, with the high statistics data sets and with the possibility of b-quark jet identification (b-tag: lifetime-tag and lepton-tag), more detailed investigations have been possible. The identification starts with the definition of jets with a jet-finding algorithm. In the commonly used DURHAM algorithm [92], (other choices are JADE [146] and LUCLUS [1]) for two particles $i$ and $j$, with energy $E_{i}$ and $E_{j}$ and opening angle $\theta_{i j}$ a value

$$
y_{i j}=\frac{2 \min \left(E_{i}^{2}, E_{j}^{2}\right)\left(1-\cos \theta_{i j}\right)}{E_{v i s}^{2}}
$$

is determined, where $E_{\text {vis }}$ is the total visible energy in the event. A pseudo-particle is formed from the pair $i$ and $j$ with smallest $y_{i j}$, its four-momentum calculated as the sum of the four-momenta of particles $i$ and $j$, and the jet-finding is iterated, until $y_{i j}$ surpasses the jet resolution parameter $y_{c u t}$ for all remaining (pseudo-) particles. The remaining pseudoparticles are called jets. The number of particles, that formed the pseudo-particle, is the particle multiplicity of the jet. Early studies at LEP by the DELPHI experiment showed higher charged particle multiplicities in multi-jet events (i.e. three or more jets) than in two-jet events [147]. A similar difference in the yield of strange particles $\left(\mathrm{K}^{0}, \Lambda^{0}, \Xi^{-}\right)$is 
observed as well. The agreement with the JETSET model is good, which follows the data as function of the resolution parameter; discrepancies are seen in two-jet events for small resolution parameters, where the JETSET prediction is higher than the data [36].

The easiest way to identify gluon jets is by jet energy ordering. In three-jet events the jet of lowest energy has the highest probability of originating from a gluon. The advent of the high precision vertex detectors opened a new era: for jets having particles with lifetime, such as B-hadron decays, one calculates the probability that the particles come from the collision point. This probability turns out to be small for b-quark jets. In three-jet events with primary b $\bar{b}$ quarks the gluon is identified as the untagged (small probabilities) jet. A second method identifies b-hadrons by the emission of leptons with high transverse momenta with respect to the beam axis. The gluon jet properties are compared to those of an unbiased three-jet sample $(1 / 3$ gluon jets, $2 / 3$ quark jets) or to events with two jets plus one photon. Topology dependences are studied with special selections: all three jets have similar energy (Mercedes events) or the two jets with the smaller opening angle have similar energy (Y events).

The results of the inner structure show a higher subjet multiplicity in gluon jets: the particles of each jet, which was found with a certain resolution parameter value $y_{c u t}$, are clustered again, but with a smaller $y_{1}$. The number of these jets within the original jets (clustered with $y_{c u t}$ ) is called subjet multiplicity. The charged particle multiplicity is higher in gluon jets than in quark jets. The increased multiplicity comes mainly from soft particles. The gluon jets are broader and show a softer fragmentation function. The details of the difference depend on the jet definition. For jets identified with the DURHAM algorithm, the four LEP experiment find consistent results for the ratio of the charged particles multiplicities in gluon and in quark jets $r=<n_{c h}^{\mathrm{g}}>/<n_{c h}^{\mathrm{q}}>=$ $1.23 \pm 0.01$. OPAL finds $1.10 \pm 0.03$ for the ratio using a cone jet finder: jets are bundles of particles, when the summed energy of these particles exceeds $10 \mathrm{GeV}$ within a cone of half opening angle of $30^{\circ}$ around the jet axis. The multiplicity is the number of particles within that cone. The numerical values are, however, significantly lower than the expected $N_{c} / C_{F}=9 / 4$ (see Section 2.3 ); or $N_{c} / C_{F} \cdot\left(1-0.27 \sqrt{\alpha_{\mathrm{s}}}-0.07 \alpha_{\mathrm{s}}\right.$ ) $\approx 2.0$ including higher order corrections [148]. From this low value one may conclude that higher order corrections, and even more non-perturbative effects, e.g., fragmentation and the event environment, are important. This is in fact confirmed by the agreement of Monte Carlo models, which include the LLA inspired parton shower evolution followed by hadronization. In addition, it has to be stressed again that the numerical values depend on the definition of the jet and the environment of the gluon and quark; on the choice of the jet-finding algorithm combining the particles and the jet resolution parameter, see References $[134,149,150,151,152,153]$ and Table 7.7.

The study of identified hadrons may give insight into the fragmentation process. A phenomenological model of a non-perturbative gluon jet [154] predicts increased production of isoscalar mesons: $\eta, \eta^{\prime}, \omega, \phi$, and states of bound glue, if they exist. Two clear tests are proposed. The $\omega / \rho^{0}$ ratio, which is close to one in quark-jets, is predicted to be large in gluon jets. The ratio exceeds three! The second easily accessible quantity is the increased yield of $\eta$ and $\eta^{\prime}$ mesons in gluon jets. An interesting quantity worth looking at may be the correlation of $\pi^{0}$ and $\eta$ in the two low-energy jets in three-jet events: 


\begin{tabular}{|l|l|l|}
\hline \multicolumn{1}{|c|}{ Topology/Method } & \multicolumn{1}{|c|}{$\mathrm{r}$} & \multicolumn{1}{c|}{ Exp. } \\
\hline Mercedes events, lifetime-tag & $1.22 \pm 0.02 \pm 0.02$ & ALEPH [151] \\
Mercedes events, lifetime-tag & $1.19 \pm 0.04 \pm 0.02$ & ALEPH [152] \\
Mercedes events $\mu /$ e-tag & $1.16 \pm 0.05$ & ALEPH [152] \\
$q \bar{q} g / q \bar{q} \gamma \mu / \mathrm{e} /$ lifetime-tag & $1.264 \pm 0.032 \pm 0.014$ & DELPHI [134] \\
Y events $\mu / \mathrm{e} /$ lifetime-tag & $1.236 \pm 0.021 \pm 0.018$ & DELPHI [134] \\
Mercedes events $\mu / \mathrm{e} /$ lifetime-tag & $1.272 \pm 0.053 \pm 0.018$ & DELPHI [134] \\
Mercedes events, correl. & $1.291 \pm 0.028 \pm 0.044$ & DELPHI [134] \\
Mercedes events lifetime-tag & $1.25 \pm 0.02 \pm 0.03$ & OPAL [153] \\
\hline Mercedes events cone & $1.10 \pm 0.02 \pm 0.02$ & OPAL [153] \\
\hline
\end{tabular}

Table 7.7: Ratio of the charged particle multiplicities in gluon and quark jets. The association of the particles to jets is performed with the DURHAM algorithm; one measurement exists using the cone algorithm. The ratio $r$ depends on the event topology. The method for the tag of the b-quark jet is given.

when a high momentum $\pi^{0}$ is found in one jet, the other will most likely contain a high momentum $\eta$ rather than a $\pi^{0}$.

The L3 collaboration has studied $\eta$ production in three-jet events [155]. The jet with lowest energy is taken as the gluon jet. With this choice the purity of quark jets is $88 \%$, the purity of gluon jets is $79 \%$. Figure 7.17 shows the $\pi^{0}$ and $\eta$ content in the identified jets as function of the scaled momentum $x_{p}$. The models correctly describe the data. In the gluon jets the $\eta$ production is enhanced, and clearly exceeds the Monte Carlo predictions. In a comparison with neutral pions in quark and gluon jets no enhanced production is observed; the Monte Carlo models describe well the spectra for all momenta in each of the three jets, both for HERwig and JETSET.

Particle identification was used by the OPAL collaboration for extracting the average number $\bar{n}_{g \rightarrow c \bar{c}}$ of gluon-splitting to $c \bar{c}$ pairs per hadronic event. This is an important ingredient for the solution of the $R_{\mathrm{b}}=\Gamma_{\mathrm{b} \overline{\mathrm{b}}} / \Gamma_{\text {had }}$ problem, considering that $\bar{n}_{g \rightarrow \mathrm{b} \overline{\mathrm{b}}} / \bar{n}_{g \rightarrow \mathrm{c} \overline{\mathrm{c}}}=$ $0.132 \pm 0.047$ (average from resummed plus leading order calculations and Monte Carlo model predictions $[156,157])$.

Two methods are used by OPAL. In the first method [156], c $\bar{c}$ are tagged with $D^{*}$ mesons. The resulting energy spectrum of these mesons is fitted to two components; direct $c \bar{c}$ production and $c \bar{c}$ pairs from gluon splitting. The second method [158] uses three-jet events. Here, the jets with lowest energy (gluon jet) containing high $p_{t}$ leptons are searched for, to tag charmed hadrons. The two results being consistent within their errors are compared to QCD calculations and model predictions in Table 7.8 [157, 158$]$.

A study, at present only possible at SLC, where the electron beam is highly polarized was performed by the SLD collaboration [160]. The average polarization was $63 \%$ and $77 \%$ for the 1993 and $1994 / 5$ runs, respectively, with the beam helicity randomly chosen between the collisions. The polar angle distribution of the initial quark is highly asymmetric. The direction of the quark follows the electron (positron) beam direction for 

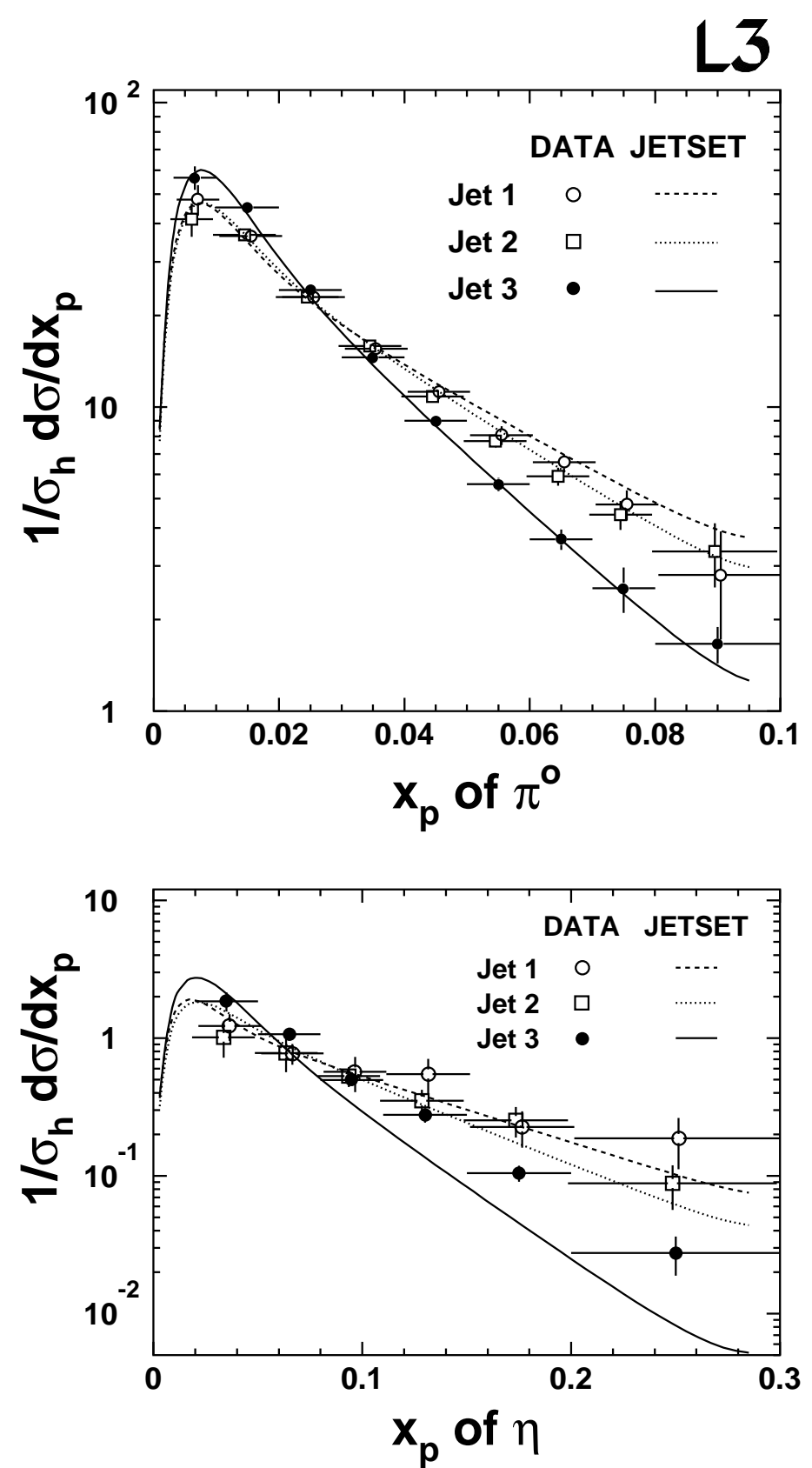

Figure 7.17: The $\pi^{0}$ and $\eta$ momentum spectrum in quark and gluon enriched jets. The data are compared with the prediction of the JETSET Monte Carlo [155].

left- (right-) handed electron beam. Thus, quark and anti-quark jets can be identified. Protons and $\Lambda$ baryons were measured separately in quark and anti-quark jets as function of momentum. Figure 7.18 shows the difference spectrum of baryon and anti-baryon: $2\left(n_{X}-n_{\bar{X}}\right) /\left(n_{X}+n_{\bar{X}}\right)$ in quark jets. At low momentum baryon and anti-baryon production are the same in both jets, while at baryon momenta above $12 \mathrm{GeV} / c$ the baryon production exceeds the anti-baryon production. This proves that fast baryons contain 


\begin{tabular}{|l|l|l|}
\hline \multicolumn{1}{|c|}{ Method } & \multicolumn{1}{|c|}{$\bar{n}_{g \rightarrow c \bar{c}}[\%]$} & \multicolumn{1}{c|}{ Ref. } \\
\hline Resummed + leading order & 1.349 & {$[157]$} \\
Leading order & 0.607 & {$[159]$} \\
JETSET & 1.701 & \\
HERwIG & 0.923 & \\
\hline$D^{*}$ & $4.4 \pm 1.4 \pm 1.5$ & OPAL [156] \\
gluon jet with $\mu \mathrm{e}-\mathrm{tag}$ & $2.27 \pm 0.28 \pm 0.41$ & OPAL [158] \\
measured average & $2.38 \pm 0.48$ & OPAL \\
\hline
\end{tabular}

Table 7.8: Average number $\bar{n}_{g \rightarrow c \bar{c}}$ of gluon-splitting to c $\bar{c}$ pairs per hadronic event. Theoretical calculations and Monte Carlo predictions are compared with the measurements.

the initial quark. With enriched b-samples and with Monte Carlo studies the SLD collaboration finds that the baryon anti-baryon difference is much smaller for heavy flavour decays.

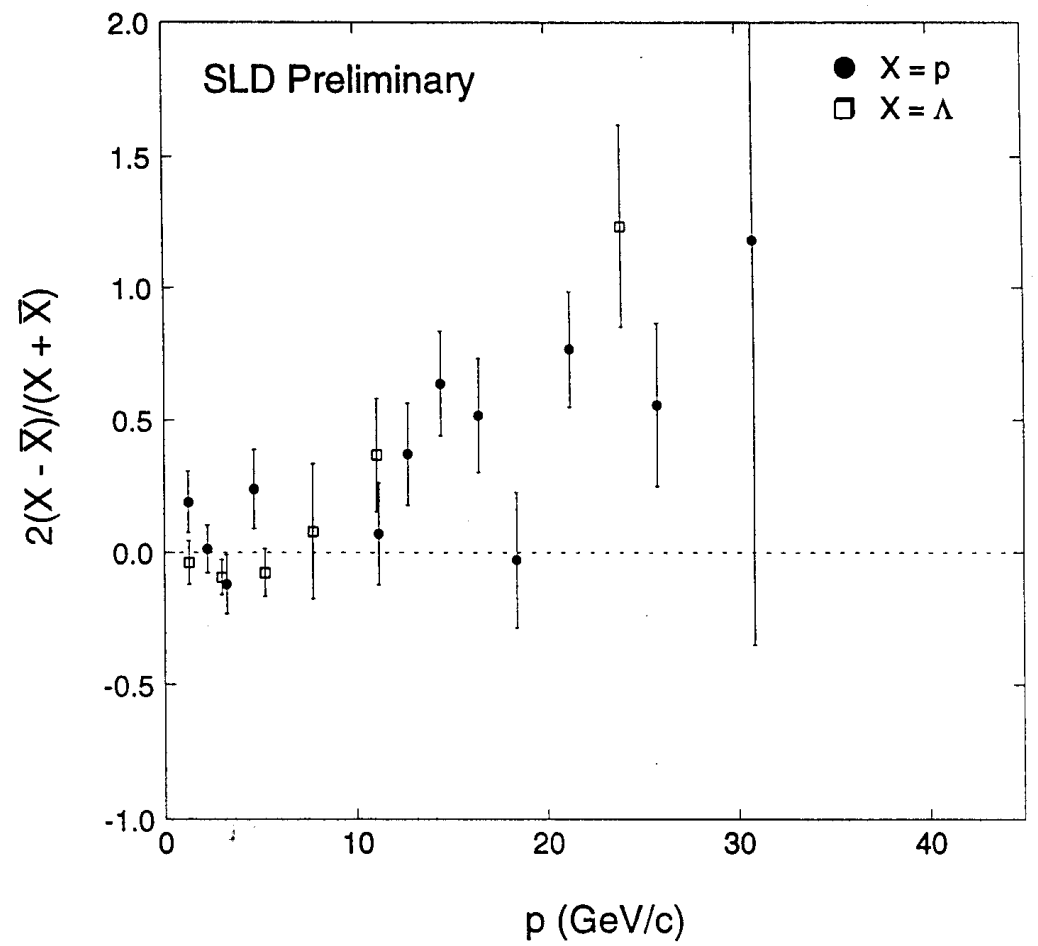

Figure 7.18: Comparison of baryon and anti-baryon production in quark jets [160]. 


\subsection{Polarization}

The large production cross-section of $\Lambda$ baryons and their clean identification in hadronic events allows the measurement of their polarization. It provides an understanding on the transfer process of polarization from the primary quarks to the final state hadron. The strange quark polarization, with respect to the quark direction, produced with unpolarized $\mathrm{e}^{+} \mathrm{e}^{-}$beams can be reliably calculated [161]. It is about -0.94 , and modified for gluon radiation it becomes -0.91 [162]. One may assume that the s-quark polarization is fully transferred to the $\Lambda$, when is contains the leading strange quark. The fraction of $\Lambda$ 's with a leading quark is measured by ALEPH from the 'hyper-charge correlation of back-toback pairs' (i.e., the two baryons are in different hemispheres) using the number of $\Lambda \Lambda$, $\bar{\Lambda} \bar{\Lambda}$, and $\Lambda \bar{\Lambda}$ pairs in data:

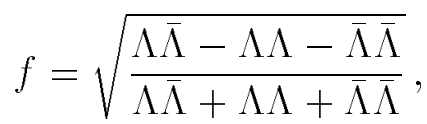

to $f=0.48 \pm 0.09(x>0.15)$, which gives the average fraction of $\Lambda$ 's from primary quarks under the assumption that the production probability for a $\Lambda$ is the same in the quark and anti-quark hemisphere. In addition, one must correct for $\Lambda$ 's, which are secondaries from heavy baryons, $\Sigma$ and $\Xi$, which at their own may be polarized. A negative final $\Lambda$ polarization around $30 \%$ for $x_{p}>0.3$ is expected.

Two experiments have measured the $\Lambda$ polarization. While ALEPH finds a polarization of $-0.32 \pm 0.07$ for $x_{p}>0.3$ [163]; DELPHI reports $0.08 \pm 0.31 \pm 0.25$ for $0.3<x_{p}<0.5$ [164].

While the DELPHI result is compatible with no polarization with large errors, the polarization as measured by the ALEPH experiment is negative and different from zero for large $x_{p}$. In Figure 7.19 the measurements are compared with the Standard Model prediction, when JETSET and HERWIG are used for the hadronization corrections (The $\Sigma^{*}$ in JETSET was reduced by $50 \%$; the normalization of the JETSET and HERWIG curves is multiplied by a factor 1.07 and 2.17, respectively, to correct for the fraction of $\Lambda$ 's with primary quark, as measured in data.). An excellent agreement is seen with the ALEPH result.

The polarization has been studied for several heavy flavoured hadrons: The decay angle of the photon in the $\mathrm{B}^{*}$ rest frame was used by the ALEPH [50] and DELPHI collaboration [51] for measuring the two helicity states. The transverse (helicity \pm 1 , $\sigma_{T} \sim\left(1+\cos ^{2} \theta^{*}\right) / 2$ ) and longitudinal (helicity $0, \sigma_{L} \sim \sin ^{2} \theta^{*}$ ) polarized states are expected to be produced with a ratio $2: 1$. The results of the two experiments agree:

$$
\begin{gathered}
\frac{\sigma_{L}}{\sigma_{L}+\sigma_{T}}=0.33 \pm 0.06 \pm 0.05, \text { ALEPH } \\
\frac{\sigma_{L}}{\sigma_{L}+\sigma_{T}}=0.32 \pm 0.04 \pm 0.03, \text { DELPHI }
\end{gathered}
$$

The DELPHI collaboration has studied the decay angle $\theta^{*}$ [53], where $\theta^{*}$ is the angle between the decay pion and the $\mathrm{B}^{* *}$ line of flight in the $\mathrm{B}^{* *}$ rest frame. The distribution is flat in $\cos \theta^{*}$, which is expected when all contributing states are unpolarized. 


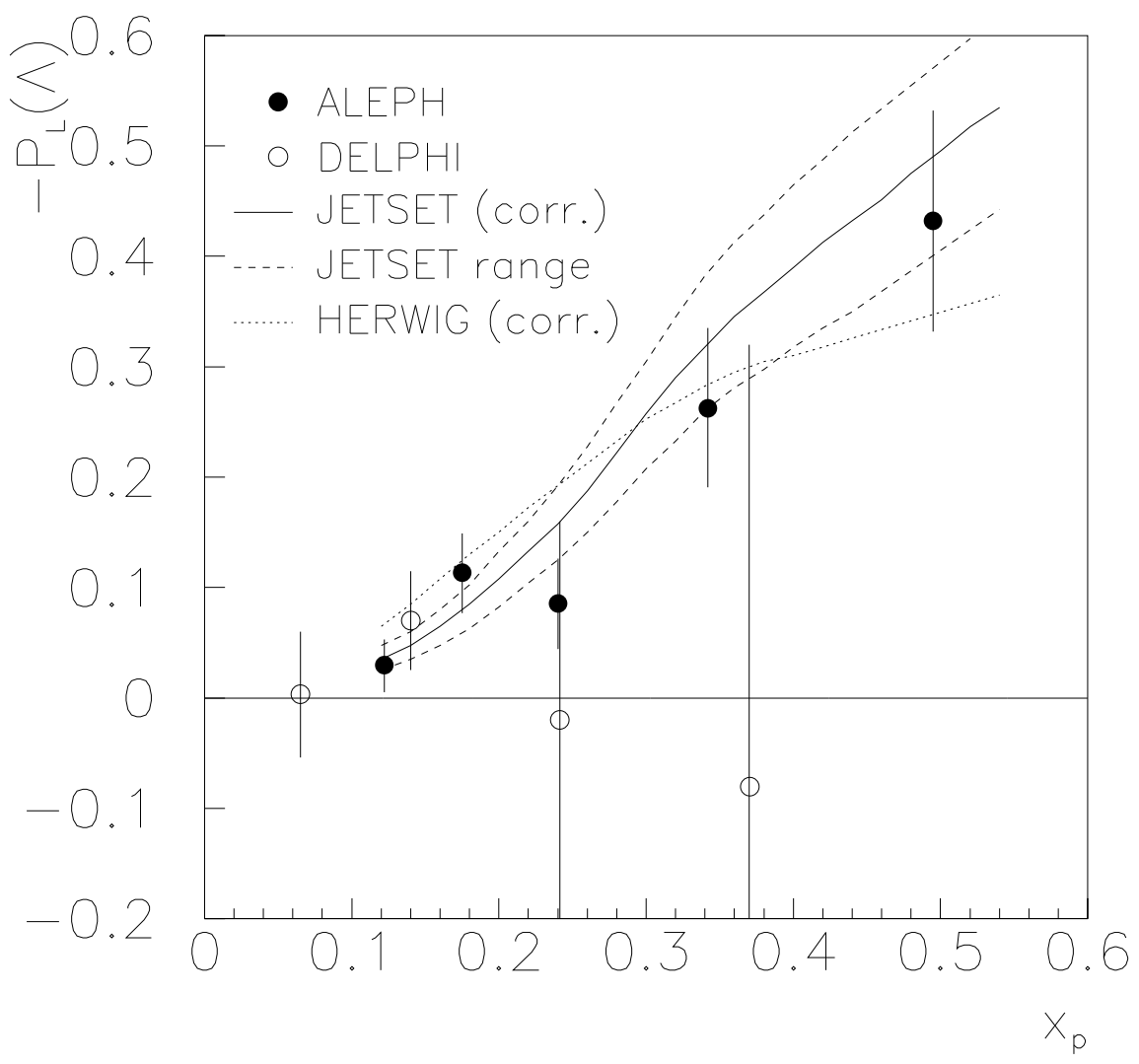

Figure 7.19: Longitudinal $\Lambda$ polarization measured with ALEPH [163] and DELPHI [164] data. The data shown with total errors are given in comparison with the JETSET model (solid line) and the estimated uncertainty (dashed line). The prediction of HERWIG is shown as dotted line. The model predictions are multiplied by factors 1.07 and 2.17, resp., as a correction for the fraction of direct $\Lambda$ 's as explained in the text.

The polarization of the $\Lambda_{\mathrm{b}}$ has been measured by the ALEPH collaboration [165] in semileptonic decays, using the property that $y=\left\langle E_{l}\right\rangle /\left\langle E_{\nu}\right\rangle$, the ratio of average charged lepton to average neutrino energy, depends on the polarization. The measurement gives:

$$
\mathcal{P}\left(\Lambda_{\mathrm{b}}\right)=-0.23_{-0.20}^{+0.24}{ }_{-0.07}^{+0.08} .
$$

This result is lower than the theoretical expectation $(-0.69 \pm 0.06)$, which assumes that a large fraction from the initial b-quark polarization of $-0.94 \%$ is transferred to the $\Lambda_{\mathrm{b}}$ baryon; only few states are known $\left(\Sigma_{\mathrm{b}}, \Sigma_{\mathrm{b}}^{*}\right)$, which may decay to $\Lambda_{\mathrm{b}}$. Other depolarization mechanisms still to be found are needed to explain the result. 


\subsection{Particle Correlations}

\section{Correlations of Distinguishable Particles}

In $\mathrm{e}^{+} \mathrm{e}^{-}$collisions, where the models reproduce rather well the single inclusive particle distributions, the study of particle correlations is a powerful technique for discriminating between models. Counting pairs of identified particle gives insight into the production mechanism. The HERWIG model, with its isotropic decay of colourless clusters, and the JETSET model, where diquarks are introduced, both suggest that study of baryon pair production is of interest. Baryons should be created in pairs, i.e., close in phase space or rapidity, for local baryon number conservation. Especially strange particles are well suited for the investigations, because the strangeness should be conserved locally as well. In Table 7.9 the measured pair-multiplicities as determined by the LEP experiments are compared.

\begin{tabular}{|c|c|c|c|}
\hline$<n>$ & ALEPH [18] & DELPHI [166, 36] & OPAL [167] \\
\hline$\Lambda \bar{\Lambda}$ & $0.093 \pm 0.009$ & $0.090 \pm 0.009$ & $0.083 \pm 0.010$ \\
$\Lambda \Lambda+\bar{\Lambda} \bar{\Lambda}$ & $0.028 \pm 0.003$ & $0.018 \pm 0.006$ & $0.021 \pm 0.005$ \\
$\Lambda \mathrm{K}_{\mathrm{s}}^{0}+\bar{\Lambda} \mathrm{K}_{\mathrm{s}}^{0}$ & $0.403 \pm 0.029$ & & \\
$\mathrm{~K}_{\mathrm{s}}^{0} \mathrm{~K}_{\mathrm{s}}^{0}$ & $0.593 \pm 0.036$ & & \\
$\Xi^{-} \bar{\Lambda}+\bar{\Xi}^{+} \Lambda$ & & $0.0085 \pm 0.0018$ & $0.0096 \pm 0.0023$ \\
$\Xi^{-} \bar{\Xi}^{+}$ & & & $0.00038 \pm 0.00067$ \\
\hline
\end{tabular}

Table 7.9: Two-particle combinations found per hadronic event with total error.

In the JETSET model an additional parameter permits additional mesons being produced between the baryons. Changing the available phase space by this popcorn mechanism (see Figure 2.7 for an illustration), also implies a change in the production rate of the particles. The rapidity difference of $\Lambda \bar{\Lambda}$ pairs shown in Figure 7.20 implies a high value of the popcorn parameter, which by default is $50 \%$.

In their model comparisons, the LEP experiments draw similar conclusions with their studies investigating correlations in different variables: polar angle, azimuth, and rapidity. The OPAL collaboration [167] for instance shows that the HERWIG model can be tuned to reproduce the inclusive rates of $\Lambda$ and $\Xi^{-}$and of the $\Lambda \bar{\Lambda}$ pairs; but the $\Sigma^{*}$ multiplicity and pairs including a $\Xi^{-}$are overestimated. This may indicate that in HERWIG a problem exists with leading baryons: $\Lambda$ 's may be produced in decays, $\Xi^{-}$baryons contain more often a leading quark. The rapidity correlation of $\Lambda \bar{\Lambda}$ pairs is too strong. This is demonstrated in Figure 7.21 [18]; with $N_{\text {had }}$ hadronic events measured, the two-particle correlations function as function of rapidity $y$ with respect to the thrust axis is defined by

$$
C\left(y_{a}, y_{b}\right)=N_{h a d} \frac{n\left(y_{a}, y_{b}\right)}{n\left(y_{a}\right) n\left(y_{b}\right)},
$$




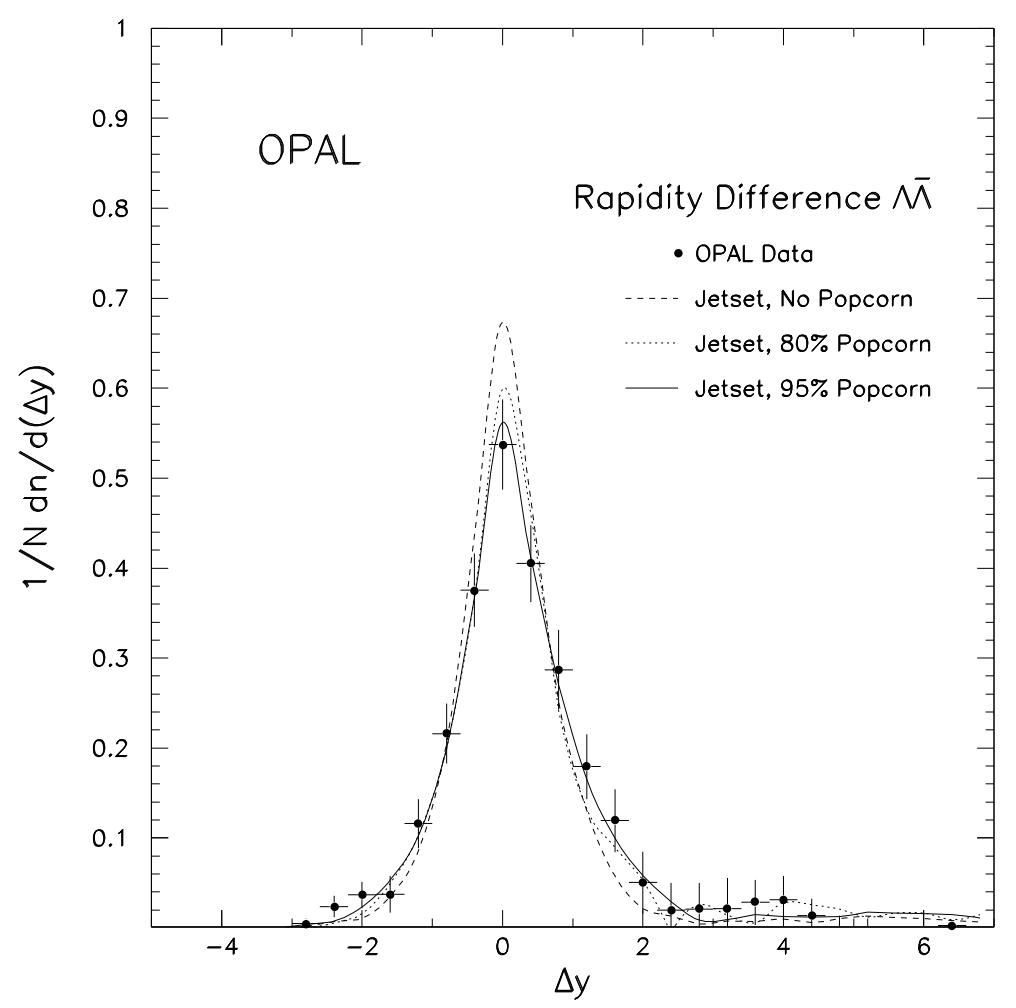

Figure 7.20: Rapidity difference of $\Lambda \bar{\Lambda}$ pairs in comparison with the JETSET model as function of the popcorn parameter [167].

where $n\left(y_{a}, y_{b}\right)$ is the joint probability density of pairs, with the particles at rapidity $y_{a}$ and $y_{b}$, and $n(y)$ is the single particle probability density.

All collaborations agree that the JETSET model with the larger number of parameters - especially the popcorn parameter and the strangeness suppression are relevant - is in better agreement with data. However, as pointed out [167], there is quite some impact on other strange baryons, especially the $\Omega^{-}$. The multiplicity of $\Lambda \bar{\Lambda}$ and $\Lambda \Lambda$ pairs prefers a popcorn parameter around $40-60 \%$ [18].

\section{Bose-Einstein Correlations}

Most studies of Bose-Einstein correlations at LEP [3] have concentrated on two-particle correlations between identical charged pions (Refs. [168] to [172]) using the quantity evaluated from the joint probability of pairs of the identical particles $\rho_{2}$ and the single particle densities $\rho_{1}$, where $k_{1}$ and $k_{2}$ are the four-momenta of the two particles:

$$
R(M)=\frac{\rho_{2}\left(k_{1}, k_{2}\right)}{\rho_{1}\left(k_{1}\right) \rho_{1}\left(k_{2}\right)} .
$$

The ratio $R(M)$ is usually given as function of $Q$ and then denoted $C(Q)$ (see Figure 

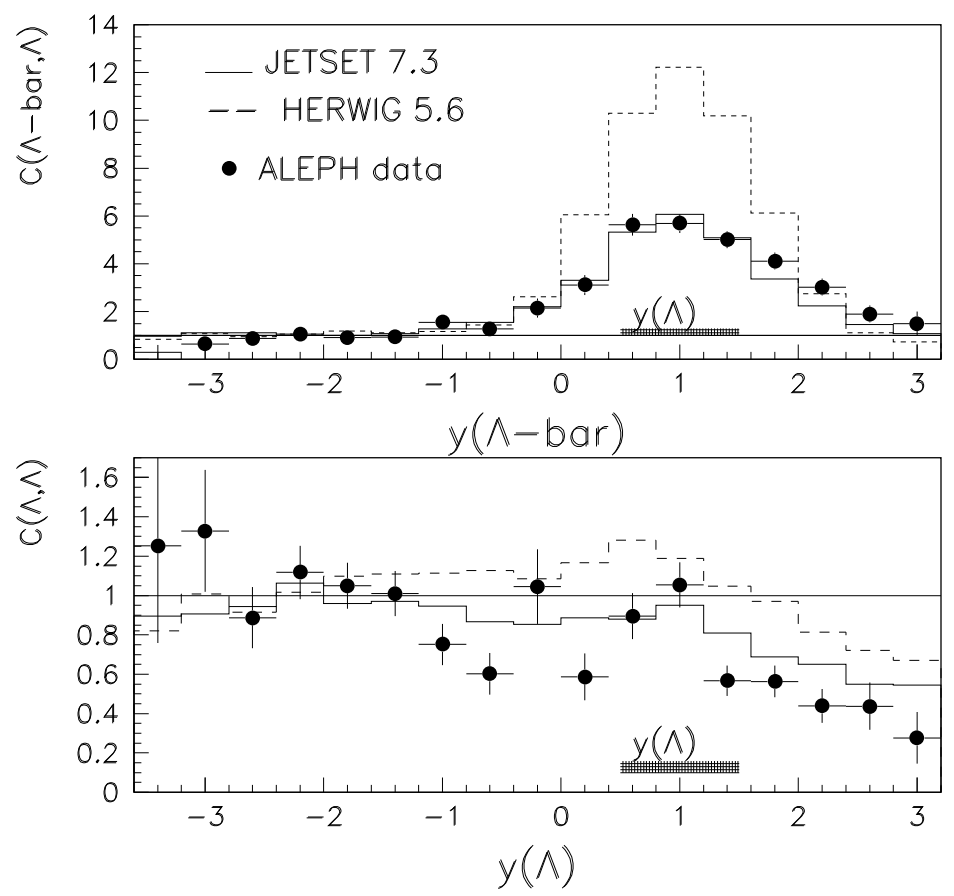

Figure 7.21: Two-particle correlation in rapidity for $\Lambda$ and $\mathrm{K}_{\mathrm{s}}^{0}$ in comparison with the JETSET and HERWIG model [18].

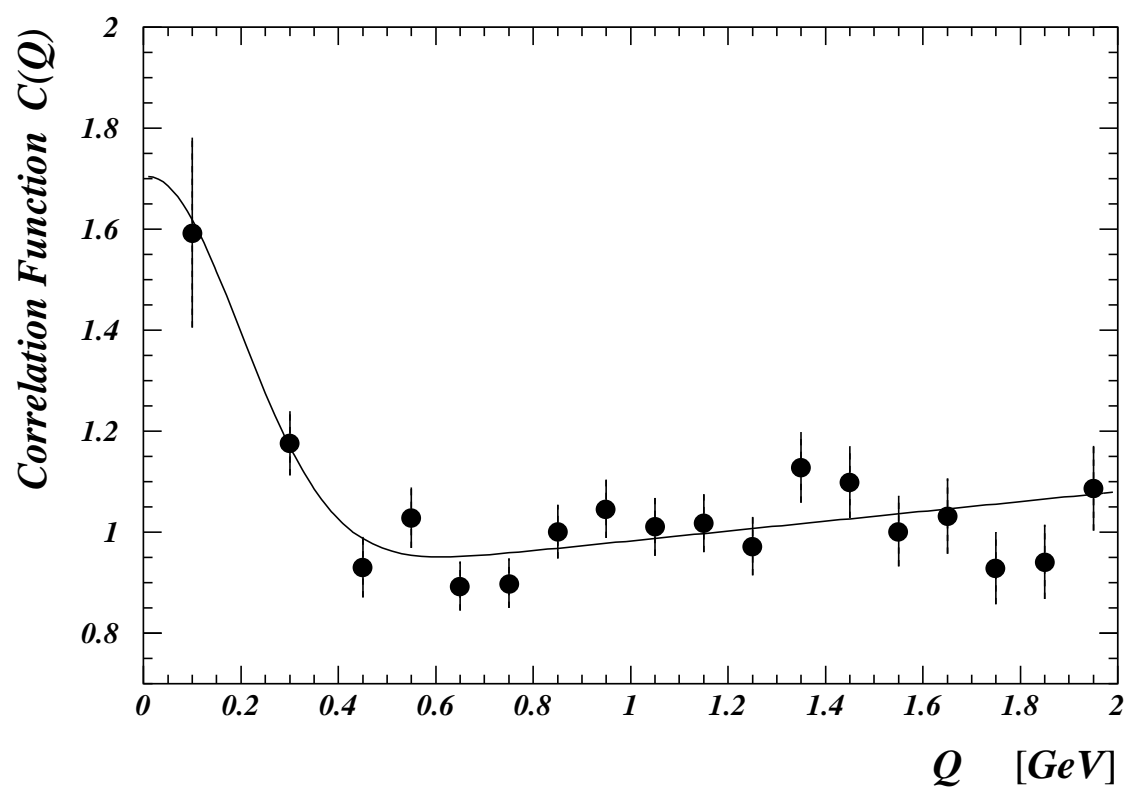

Figure 7.22: Correlation function $C(Q)$ for neutral kaons [20]. 
7.22 for an example with kaons), $Q^{2}=M^{2}-4 m_{\pi}^{2} ; M$ is the invariant mass of the pair. The denominator $\rho_{1}\left(k_{1}\right) \rho_{1}\left(k_{2}\right)$ is obtained from a reference sample. This sample should resemble $\rho_{2}\left(k_{1}, k_{2}\right)$ except for the Bose-Einstein correlations being studied.

Two choices for the reference sample are made, unlike-sign pion pairs or uncorrelated pairs from track mixing. Both alternatives have their disadvantages. Unlike-sign pion pairs suffer from correlations due to resonances not present in like-sign pion pairs, and the contribution of resonances, esp. $\eta$ and $\eta^{\prime}$ at low $Q$, with rates not well known. The track mixing has the disadvantage that correlations, other than from Bose-Einstein correlations, are missing. In addition, cuts are necessary to suppress the effects of gluon radiation. For both methods, systematic uncertainties are reduced using the double ratio $R^{\text {data }}(M) / R^{\mathrm{MC}}(M)$. Additional corrections for background and for Coulomb interactions are applied.

The two-particle correlation function $R(M)$ is related to the four-dimensional Fourier transform of the density distribution for the source of the particles, allowing the study of the size of the emitting source. Assuming a spherical and Gaussian source the enhancement at low $Q$ is parameterized as

$$
R(M) \sim 1+\lambda \exp \left(-r^{2} Q^{2}\right)
$$

The chaoticity parameter $\lambda$ is expected to vary between 0 and 1 , and is extracted from data in the range from 0.4 to 1.5 ; the radius $r$ of the source is measured $0.4 \mathrm{fm}$ to $1.0 \mathrm{fm}$. In Figure 7.23 and Table 7.10 the background-corrected measurements are displayed for the meson pairs $\pi^{ \pm} \pi^{ \pm}, \pi^{0} \pi^{0}, \mathrm{~K}^{ \pm} \mathrm{K}^{ \pm}$, and $\mathrm{K}^{0} \mathrm{~K}^{0}$. (See as well [173] for a recent summary.)

Only identical mesons that do not originate from long-lived resonances (prompt mesons), can contribute to the enhancement at low $Q$. It has been pointed out that the measured value of $\lambda$ is about the maximum expected from direct pairs or is even higher [174].

In more recent analyses the fraction $f(Q)$ of non-direct pions as function of $Q$ has been parameterized using Monte Carlo and included in the fit. For example, DELPHI uses $f(Q)=0.17+0.26 Q-0.12 Q^{2}$ to fit $\lambda$ and $r$ for charged pions:

$$
R(M) \sim 1+\lambda f(Q) \exp \left(-r^{2} Q^{2}\right) .
$$

While the change in the radius is small, $\lambda$ is changed by a factor 3 . A larger change is reported by L3 for $\pi^{0} \pi^{0}$ correlations [175]. The corrections are very sensitive to the model used. The corrections for non-prompt mesons are indicated by arrows in Figure 7.23.

Kaon pairs have higher chaoticity values than pions before correction $[18,20,176]$. Only DELPHI has estimated the corrections for non-prompt kaons. The correction for kaons from $c$ - and b-decay increases $\lambda$ by $\approx 25$ to $30 \%$.

Bose-Einstein correlation affects indirectly also the mass spectra of unlike-sign pion pairs. In the invariant mass distribution of pions the $\rho^{0}$ meson appears shifted towards lower masses $[19,23,26,170,177,178]$. In the framework of the JETSET model (see Figure 7.24), where Bose-Einstein correlations are implemented as final state interaction, introducing $R(M) \sim 1+\lambda \exp \left(-r^{2} Q^{2}\right)$ into the generator, this can be interpreted as coming from Bose-Einstein correlations, which induce correlations between unlike-sign combinations. OPAL finds a reasonable agreement between data and JETSET including 


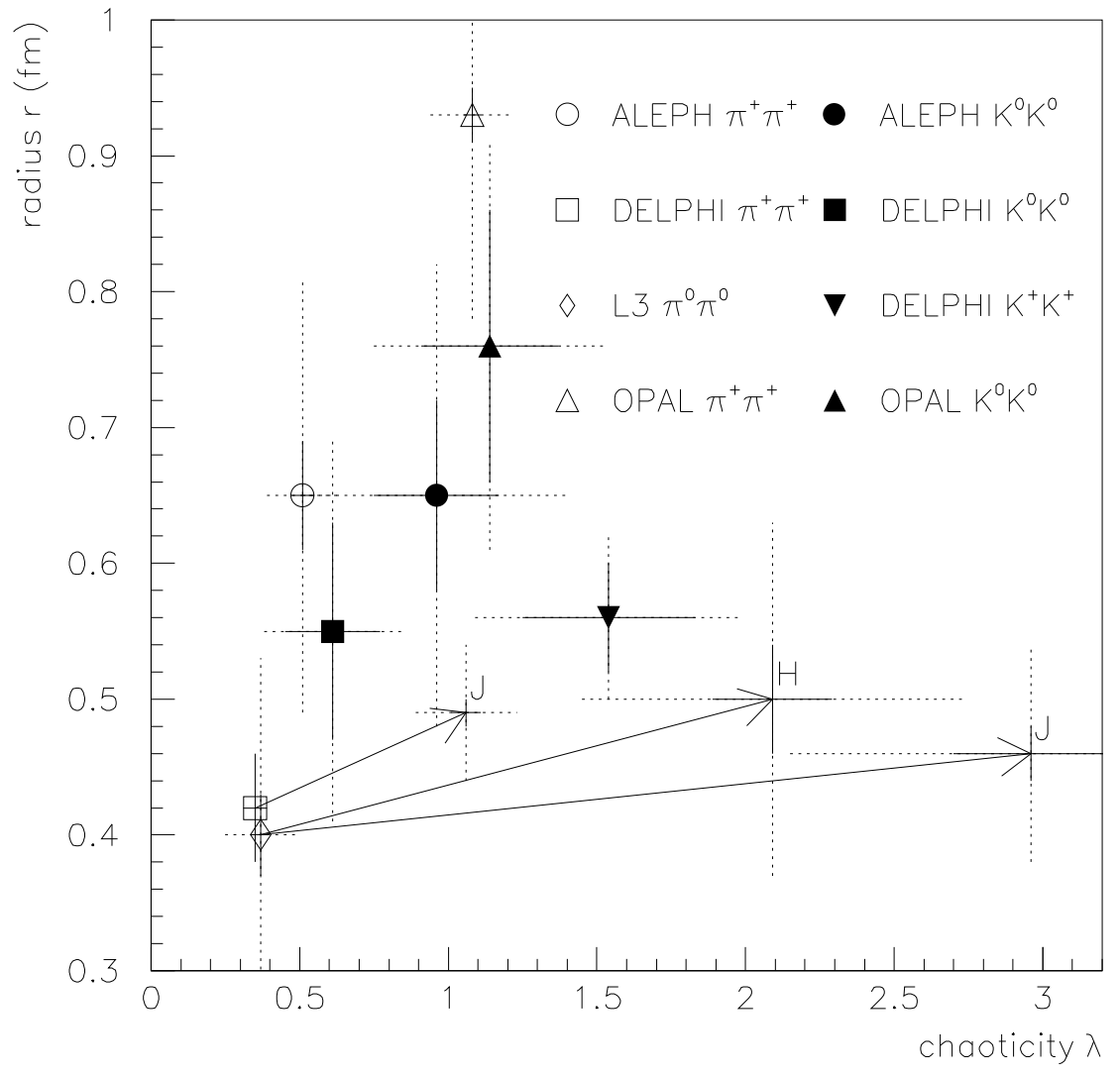

Figure 7.23: Chaoticity parameter $\lambda$ versus radius $r$ measured at LEP. Measured values, corrected for background, with statistical (solid line) and total errors (dots) are shown. The arrows indicate the changes, when corrected for non-prompt meson-pairs estimated with JETSET or HERWIG, when it is calculated by the experiment and given in the publication [3].

Bose-Einstein correlation, when the chaoticity parameter is set to 2.5. This value of $\lambda$ was obtained with a fit to the ratio $R(M)$. ALEPH agrees with this observation and extracts a $\rho^{0}$ rate with $\lambda$ and $r$ as free parameters. The value of $\lambda=2.1$ is compatible with OPAL in view of the different $\eta^{\prime}$ rate and choice of the coherence time parameter $\chi \cdot(\chi$ gives the minimum width of resonances whose daughters contribute to the Bose-Einstein enhancement; chosen to be $20 \mathrm{MeV} / c^{2}$ by OPAL, $100 \mathrm{MeV} / c^{2}$ by ALEPH.) DELPHI, which observes a shift of the $\rho^{0}$, uses its $\lambda$ value extracted from the Bose-Einstein analysis, after correction, for the $\rho^{0}$ analysis.

Three-particle correlations have been studied by DELPHI (Figure 7.25). While JETSET without Bose-Einstein correlations fails to describe the data, JETSET including BoseEinstein correlations gives a fair description of unlike-sign triplets; the shape is reproduced, but the magnitude is somewhat low [179].

Concerns have to be raised about the implementation of Bose-Einstein correlations in JETSET. The implementation treats them as a classical force, which violates energy- 


\begin{tabular}{|c|c|c|c|}
\hline particles & $\lambda$ & $r[\mathrm{fm}]$ & Exp. \\
\hline$\pi^{ \pm} \pi^{ \pm}$ & $0.51 \pm 0.04 \pm 0.22$ & $0.65 \pm 0.04 \pm 0.16$ & ALEPH [168] \\
\hline$\pi^{ \pm} \pi^{ \pm}$ & $0.35 \pm 0.04$ & $0.42 \pm 0.04$ & DELPHI $[170]$ \\
\hline$\pi^{ \pm} \pi^{ \pm}($prompt, JS $)$ & $1.06 \pm 0.05 \pm 0.16$ & $0.49 \pm 0.01 \pm 0.05$ & DELPHI [170] \\
\hline$\pi^{ \pm} \pi^{ \pm}$ & $1.08 \pm 0.05 \pm 0.14$ & $0.93 \pm 0.05 \pm 0.24$ & OPAL $[172]$ \\
\hline$\pi^{0} \pi^{0}$ & $0.37 \pm 0.03 \pm 0.12$ & $0.40 \pm 0.03 \pm 0.05$ & L3 $[175]$ \\
\hline$\pi^{0} \pi^{0}$ (prompt,JS) & $2.96 \pm 0.26 \pm 0.77$ & $0.46 \pm 0.02 \pm 0.08$ & L3 $[175]$ \\
\hline$\pi^{0} \pi^{0}$ (prompt,HW) & $2.09 \pm 0.20 \pm 0.61$ & $0.50 \pm 0.04 \pm 0.12$ & L3 [175] \\
\hline $\mathrm{K}^{ \pm} \mathrm{K}^{ \pm}$ & $1.54 \pm 0.29 \pm 0.35$ & $0.56 \pm 0.04 \pm 0.04$ & DELPHI [171] \\
\hline $\mathrm{K}^{0} \mathrm{~K}^{0}$ & $0.96 \pm 0.21 \pm 0.40$ & $0.65 \pm 0.07 \pm 0.15$ & ALEPH [18] \\
\hline $\mathrm{K}^{0} \mathrm{~K}^{0}$ & $0.61 \pm 0.16 \pm 0.16$ & $0.55 \pm 0.08 \pm 0.12$ & DELPHI [171] \\
\hline $\mathrm{K}^{0} \mathrm{~K}^{0}$ & $1.14 \pm 0.23 \pm 0.32$ & $0.76 \pm 0.10 \pm 0.11$ & OPAL $[20]$ \\
\hline
\end{tabular}

Table 7.10: Chaoticity parameter $\lambda$ and radius $r$ measured at LEP. Measured values, corrected for background, are given. Some measurements have been corrected for nonprompt hadrons using the the JETSET(JS) or HERWIG(HW) model.

momentum conservation. The rescaling, however, twists the event shape variables; the model tuning becomes worse $[97,98,99]$. Studies on a modified implementation, which also moves unlike-sign pairs to avoid rescaling, improves the situation. Both like-sign and unlike-sign spectra get a counter-weight $1 / E(M)$, with

$$
E(M) \sim 1+\epsilon \lambda \exp \left(-r^{2} Q^{2}\right)
$$

The additional $\epsilon$ parameter should be the fraction of like-sign pairs $\approx 0.1$. Various parameter settings have been studied. A small influence on the event shape variables is found with a high $\epsilon(\approx 0.3)$ and a high $\lambda(\approx 2)$ parameter. The mass shift of the $\rho^{0}$ is not reproduced with this parameter setting [99].

Another new simulation, based on the area spanned by the string, is in preparation. A first result with a toy Monte Carlo predicts that the reconstructed $\lambda$ should be 2 for $\pi^{0}$, when $\lambda=1$ is used for event generation [180].

At first glance, the experimental results are different, $\lambda \approx 1$ for corrected direct measurements (DELPHI), and $\lambda \approx 2$ for an extraction tuning the JETSET model. However, the following differences must be kept in mind: The use of track mixing as reference distribution tends always to give lower $\lambda$ values than the use of the unlike-sign meson sample. The uncorrected values for DELPHI are lower than for the other experiments. For kaons, corrections are estimated for c- and b-decays only, but not for strong decays. ALEPH has used daughters of resonances wider than $\Gamma=100 \mathrm{MeV} / c^{2}$ as prompt pions, excluding the $\mathrm{K}^{*}$, which seems to be un-affected by Bose-Einstein correlations. Ignoring this and correcting OPAL measurements for the $\eta^{\prime}$ rate would bring the values down to $\lambda=1.7$ in these two analyses.

More understanding is needed, how to include the correlation without twisting the event shape distribution. The new $\epsilon$ parameter improves the situation; but there is no 


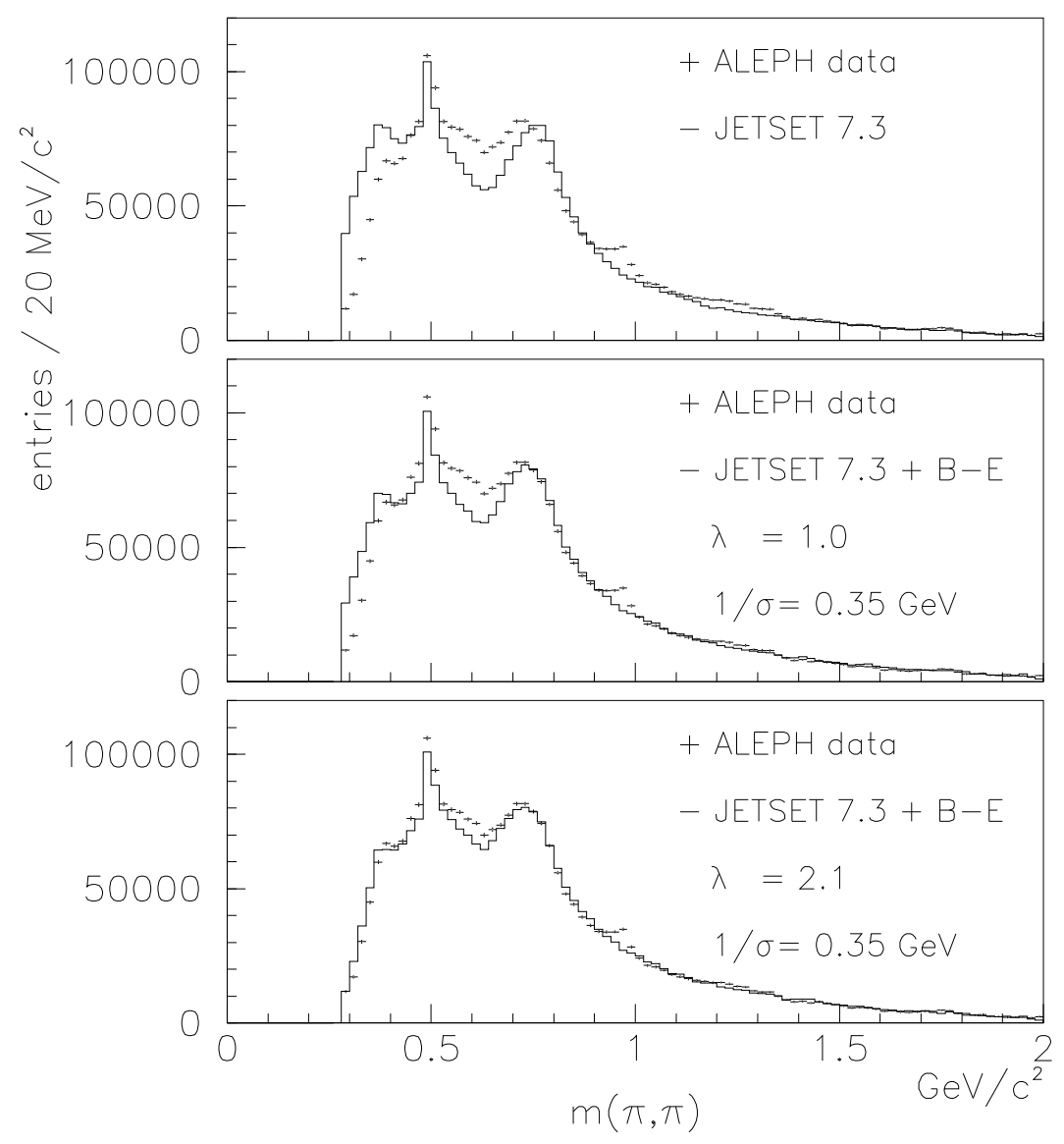

Figure 7.24: Subtracted two-pion mass spectra (unlike minus like-sign) for data (crosses) and JETSET (solid curve) with and without Bose-Einstein correlations are shown, including detector simulation [23].

real success yet. Taking the decay amplitudes, i.e. string area, may be another promising approach.

No studies have been performed yet, using information of the event topology. The particle multiplicity should have an influence. Even more important, investigations should look separately for correlations in quark and gluon jets [180]. This should be sensitive to the production mechanism of identical particles in the string; naively, two strings are spanned from a gluon, but only one from a quark. In a recent paper [181] the MD1 collaboration has compared its data at the $\Upsilon$, i.e., 3 -gluon or gg $\gamma$ decays, with its continuum events $(q \bar{q})$ at $\sqrt{s}=7.2$ to $10.3 \mathrm{GeV}$. They find no noticeable difference. A similar conclusion is drawn by the CLEO collaboration [182]. The data taken with the MARK II detector at the $\mathrm{J} / \psi$ resonance gave a $\lambda$ close to the maximum expected value of 1 , while for the continuum they find about half this value [183].

A greater understanding is important for future studies: recently it has been pointed out that a shift of the W-mass, measured in the hadronic decays of the $\mathrm{W}$-pair $\mathrm{e}^{+} \mathrm{e}^{-} \rightarrow$ 

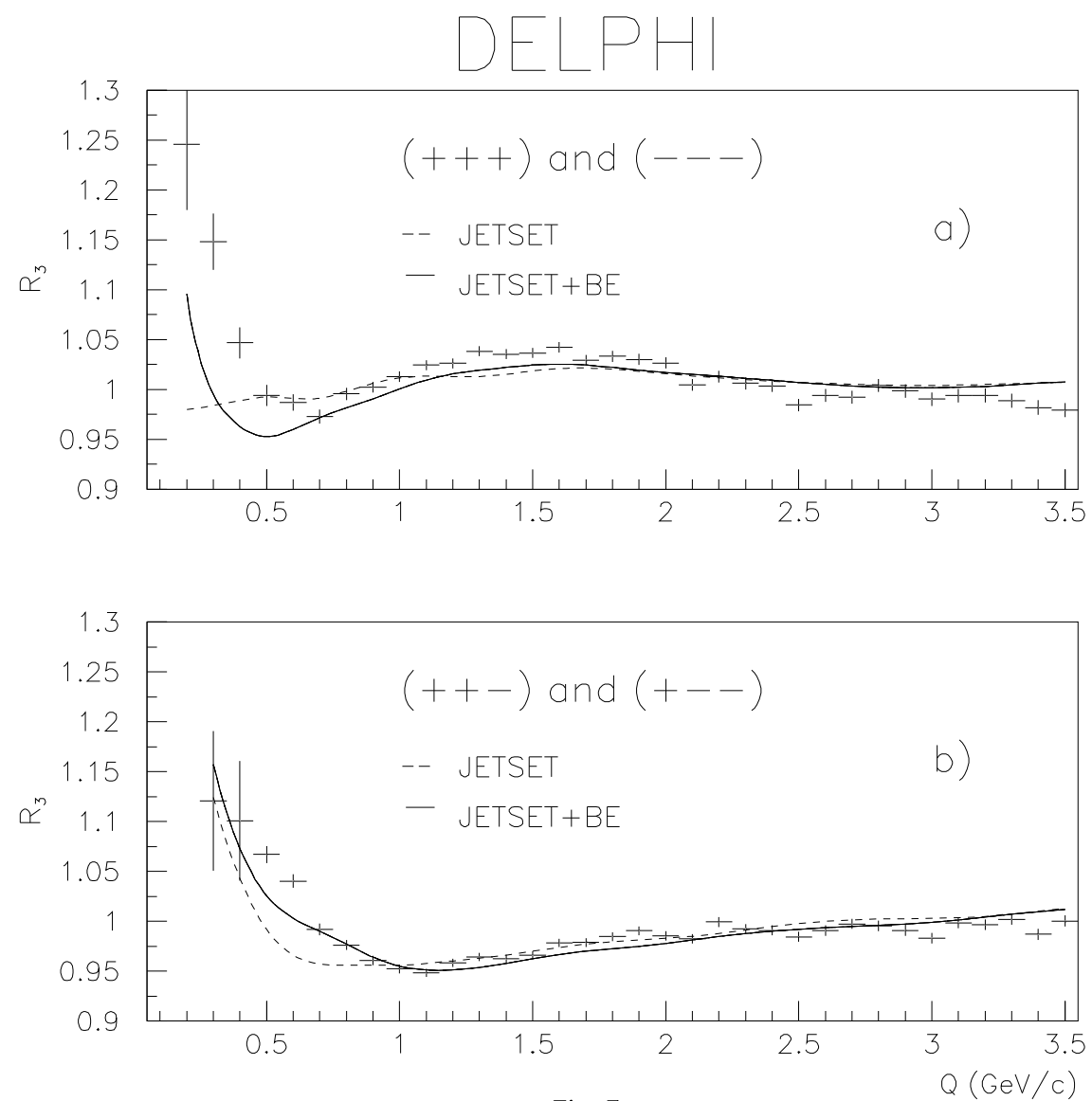

Fig.3

Figure 7.25: Three-particle correlation function for like-sign triplets and unlike-sign triplets extracted with data of the DELPHI detector. Comparison with JETSET are given without and with Bose-Einstein correlations enabled: $\lambda=1$. and $r=0.50 \mathrm{fm}$; the $\eta^{\prime}$ rate was reduced by a factor four to fit the data [179].

$\mathrm{q}_{1} \overline{\mathrm{q}}_{2} \mathrm{q}_{3} \overline{\mathrm{q}}_{4}$, may be as large as $100 \mathrm{MeV} / \mathrm{c}^{2}$ [184]. The reason is due to Bose-Einstein correlations; the decay vertices of $\mathrm{W}^{+}$and $\mathrm{W}^{-}$are closer than the hadronization distances and the size of the source of Bose-Einstein correlations. 


\section{Chapter 8}

\section{Conclusion and Outlook}

The four LEP collaborations have measured the inclusive production of about 30 hadrons. With the large event statistics of approximately 20 million events in Z decays taken around $\sqrt{s}=91 \mathrm{GeV}$, they measured particles with production probabilities different by several orders of magnitude: about 17 charged pions, but $1.5 \cdot 10^{-4} \Upsilon$ are produced per hadronic event. The momentum spectra have been measured for many particles over most of the $x_{p}$ range with higher precision than at previous $\mathrm{e}^{+} \mathrm{e}^{-}$colliders. The rates of mesons are known with an accuracy of a few percent, those of baryons with $10-20 \%$.

The measurements comprise the light pseudoscalar and vector mesons, and the lowest mass baryon octet and decuplet. In each of these multiplets at least one state for each isospin has been measured; usually the particles with a $\pi^{0}$ in the final state are not measured. Charm and bottom hadrons have been studied, as well as some mesons with

orbital angular momentum; but no excited baryons, with the exception of the $\Sigma_{\mathrm{b}}^{*}$, have been seen.

Results have been compared with shower models, tuned to global event shape variables, particle momentum spectra and their average multiplicities. The QCD predictions, both perturbative and non-perturbative aspects, together with hadronization models are implemented in these models using Monte Carlo simulation, and provide a convenient means of comparison. The particle production rates predicted by the coherent shower models, JETSET and HERWIG, are in good agreement for the mesons in most cases; small differences do exist, e.g., for charged kaons.

For baryons the concept of diquark production is favoured by the data. The agreement with models is worse than for mesons. The HERWIG model overestimates the production of strange baryons, and two-particle correlations are too strong. The JETSET model describes the data better; problems remain, however, such as a simultaneous description of the proton and $\Lambda$ spectrum. Correlation studies favour the popcorn mechanism implemented in the JETSET model, which allows for production of a meson between two baryons. However, an exact description of rates and correlations is still not available. The $\Omega^{-}$, which was measured with a high rate at PEP/PETRA energies, compared to the JETSET model, is now in good agreement. Bose-Einstein correlations are still not really understood and need further investigation.

Predictions of the modified leading logarithmic approximation are confirmed. When 
studying the position $\xi^{*}$ of the maximum of the $\xi=\ln \left(1 / x_{p}\right)$ distribution, where the modified leading logarithmic approximation is combined with local parton hadron duality, it turns out, however, that corrections for resonance decays are important.

Phenomenological models, which have only a few parameters, in contrast to the elaborate Monte Carlo models, give a fair description of particle production rates in data and show some general dependences in particle production (spin, isospin, mass), but the physical insight that such models might provide is not clear. The Monte Carlo models JETSET and HERWIG, which are used for understanding the details of the fragmentation process, are in better agreement. However, the agreement is probably partially due to the higher number of free parameters, which is unsatisfactory and leaves room for improvements.

Further studies are needed to understand the production of $L=1$ mesons. They have a large production rate and may provide an insight on the transition from the fragmentation region to the decay regime. Some particles, where experiments disagree on their measurement such as the $\Delta^{++}$baryon and high momentum protons, require further investigation.

The disagreement in some particle production rates and spectra between experiments $\left(\Delta^{++}, \eta^{\prime}\right)$, as well as the incomplete simulation of particle correlations and rates (strange baryons, $L=1$ mesons) point to the fact that many processes are not yet understood. The situation may be improved; many investigations used only part of the available data and with the full statistics more specific aspects may be looked at. Variables other than $x_{p}$, such as $p_{t}^{\text {out }}$, may be studied for more insight into the fragmentation process. Studies on Bose-Einstein correlations in gluon jets will test the string concept. The study of particle composition in gluon jets, which has just started, will continue; studies on hadrons in jets with specific quark-flavour are to follow. None of these measurements has been used for standard model tunings; first investigations had shown that adjusting two-particle distributions has a considerable impact on event shape distributions.

In the near future, when the LEP collider runs above the $\mathrm{W}^{+} \mathrm{W}^{-}$threshold, the main topics will be the $\mathrm{W}^{ \pm}$mass measurement and searches for new particles. QCD events will be one of the main backgrounds and will therefore have to be understood quite well; the influence of Bose-Einstein correlations on the $W$-mass measurement in four-jet events may be significant. Studies have been done extrapolating various models with the different tunings of the four experiments to LEP II energies [3]. Measurements of event shape distributions and particle spectra will constrain the models. Though the event statistics will be much lower than at LEP I, errors will be similar to the ones at PEP/PETRA. Multiplicity measurements are often dominated by systematics, e.g., stable particles and $\mathrm{V}^{0}$ s, and the results will be compatible in precision with those from LEP I. 


\section{Acknowledgements}

It is a pleasure to thank all who have contributed to this review; the personnel of the LEP accelerator and the members of the four LEP collaborations, especially my colleagues within the ALEPH experiment.

Special thanks go to Prof. S. Brandt, Prof. C. Grupen, Dr. G. Rudolph, Prof. F. Bopp and Dr. I. Dawson for their support, fruitful discussions, suggestions and comments.

I enjoyed working at the Physics Department at Siegen University within the ALEPH group and with students, the nice atmosphere, help and encouragement, table-tennis matches and much more. 


\section{List of Figures}

1.1 Cross section in $\mathrm{e}^{+} \mathrm{e}^{-}$annihilation as a function of centre-of-mass energy $\quad$ - 2

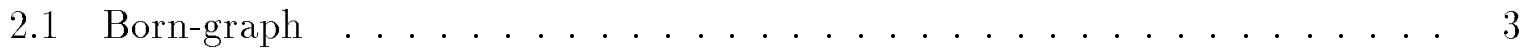

2.2 Inclusive single-hadron production . . . . . . . . . . . . . 5

2.3 Compilation of measurement demonstrating the running of the strong cou-

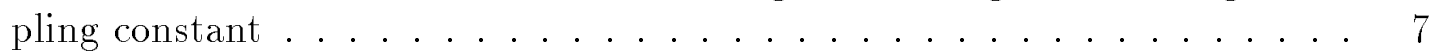

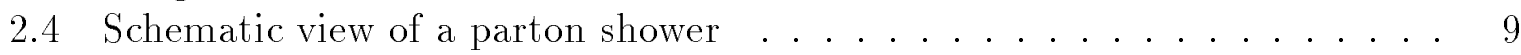

2.5 Comparison of fragmentation functions . . . . . . . . . . . . 11

2.6 Illustration of the string and cluster fragmentation . . . . . . . . . . 11

2.7 Space-time diagrams illustrating baryon production in the string model . . 13

3.1 LEP storage ring . . . . . . . . . . . . . . . . . . 18

3.2 Integrated luminosity seen by the LEP experiments . . . . . . . . . . 20

3.3 ALEPH detector . . . . . . . . . . . . . . 23

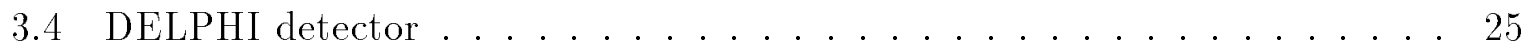

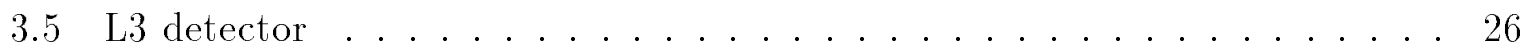

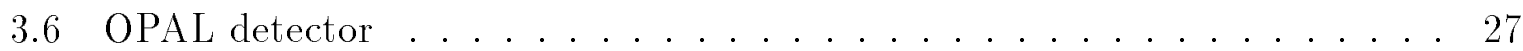

3.7 Measured $n$-jet rates . . . . . . . . . . . . . . . . 28

3.8 Energy and particle flow in three-jet events $(\mathrm{q} \overline{\mathrm{q}} \gamma$ and $\mathrm{q} \overline{\mathrm{q} g}) \ldots \ldots . . .29$

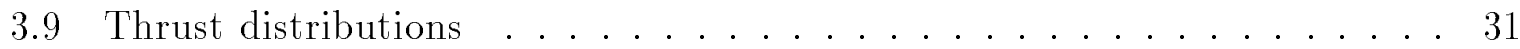

$3.10 p_{t}^{\text {in }}$ and $p_{t}^{\text {out }}$ distributions . . . . . . . . . . . . . . . 31

3.11 Energy versus multiplicity of charged particles . . . . . . . . . . . 34

4.1 Particle identification with the DELPHI detector .......... 36

4.2 Invariant mass spectrum $m\left(\mathrm{~K}^{ \pm} \pi^{\mp}\right) \ldots \ldots \ldots$. . . . . . . . 38

5.1 Multiplicity ratios of particle production rates at different $E_{c m s} \ldots \ldots 44$

6.1 Energy loss as function of momentum $x_{p}=p / p_{\text {beam }} \ldots \ldots$. . . . . 49

6.2 Cherenkov angle measured with the liquid radiator . . . . . . . . . . 50

6.3 Momentum spectra in $x_{p}$ for charged particles, $\pi^{ \pm}, \mathrm{K}^{ \pm}$, and $(\mathrm{p}, \overline{\mathrm{p}}) \ldots \ldots$. . 51

6.4 Momentum spectra in $\xi$ for charged particles, $\pi^{ \pm}, \mathrm{K}^{ \pm}$, and $(\mathrm{p}, \overline{\mathrm{p}}) \ldots \ldots 2$

6.5 Fraction of pion, kaon, and proton of all charged particles . . . . . . . 53

6.6 Separation of $\Lambda$ and $K_{\mathrm{s}}^{0}$ in the Armenteros-plot. . . . . . . . . . . 55

6.7 Momentum spectra for $\mathrm{K}_{\mathrm{s}}^{0}$ and $\Lambda \ldots \ldots \ldots \ldots$

6.8 Transverse momentum spectra for $\mathrm{K}_{\mathrm{s}}^{0}$ and $\Lambda \ldots \ldots \ldots$ 
6.9 Momentum spectra for $\pi^{0}, \eta$, and $\eta^{\prime} \ldots \ldots \ldots \ldots$. . . . . . . . . . . . . . 60

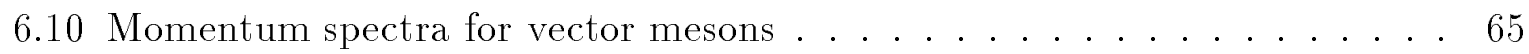

6.11 Momentum spectra for $f_{0}, f_{2}$, and $f_{2}^{\prime} \ldots \ldots \ldots \ldots$. . . . . . . . . . . . . . . .

6.12 Momentum spectra for $\Delta^{++} \ldots \ldots \ldots \ldots \ldots$. . . . . . . . . . . . . . . . . . . 70

6.13 Momentum spectra for strange baryons . . . . . . . . . . . . . . 74

6.14 Momentum spectra for $\mathrm{D}^{* \pm} \ldots \ldots \ldots \ldots \ldots$. . . . . . . . . . . . . . . . . . . 77

6.15 Momentum spectra for $\mathrm{D}_{\mathrm{s}}^{+} \ldots \ldots \ldots \ldots \ldots \ldots$. . . . . . . . . . . . . . . . . . . . . . . . . .

6.16 Measurements of $\left\langle x_{E, c}\right\rangle$ for charm quarks . . . . . . . . . . . 79

6.17 Momentum spectra for $\mathrm{J} / \psi \ldots \ldots \ldots \ldots \ldots$. . . . . . . . . . . . . . . . . . . . 82

6.18 Momentum spectra for B-hadrons . . . . . . . . . . . . . . 85

6.19 Measurements of $\left\langle x_{E, b}\right\rangle$ for bottom quarks . . . . . . . . . . 86

7.1 Particle multiplicity in the Chliapnikov-model . . . . . . . . . 87

7.2 Particle multiplicity in the uncorrelated thermodynamical model . . . . . 89

7.3 Particle multiplicity in the correlated thermodynamical model . . . . . . . 89

7.4 Different models for baryon production . . . . . . . . . . . . . 91

7.5 Strangeness suppression: Data with model comparisons . . . . . . . . 93

7.6 Strangeness suppression with corrections for particle decays . . . . . . . 93

7.7 Spin suppression: Data with model comparisons . . . . . . . . . . 96

7.8 Spin suppression with corrections for particle decays . . . . . . . . . 96

$7.9 \xi^{*}$-dependence on hadron mass . . . . . . . . . . . . . . 98

7.10 Multiplicity distribution as function of $z=n_{c h} /\left\langle n_{c h}\right\rangle$ (KNO-scaling). . 99

7.11 Charged multiplicity as function of the effective centre-of-mass energy in

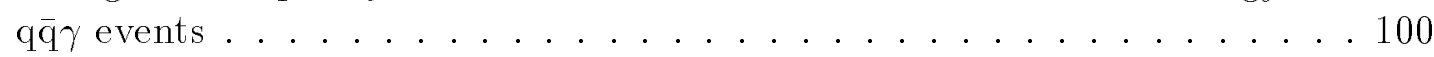

$7.12 \xi^{*}$ for charged particles as function of the centre-of-mass energy . . . . . 101

$7.13 \xi^{*}$ for neutral mesons as function of the centre-of-mass energy . . . . . . 102

7.14 Inclusive charged particles energy distribution at various centre-of-mass

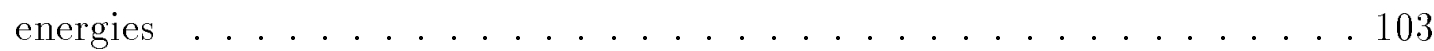

7.15 Ratio of inclusive energy distribution of ALEPH and TASSO . . . . . . 104

7.16 Difference of the charged multiplicity of $c$ - and b-flavoured jets to light

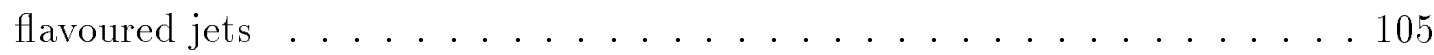

7.17 The $\pi^{0}$ and $\eta$ momentum spectrum in quark and gluon enriched jets . . . 109

7.18 Baryon and anti-baryon production in quark jets . . . . . . . . 110

7.19 Longitudinal $\Lambda$ polarization . . . . . . . . . . . . . . . . . . . . . . . . . . . . . .

7.20 Rapidity difference of $\Lambda \bar{\Lambda}$ pairs . . . . . . . . . . . . . . . 114

7.21 Two-particle correlation in rapidity for $\Lambda$ and $\mathrm{K}_{\mathrm{s}}^{0} \ldots \ldots \ldots \ldots$

7.22 Correlation function $C(Q)$ for neutral kaons $\ldots \ldots \ldots \ldots$

7.23 Bose-Einstein correlations: Chaoticity parameter $\lambda$ versus radius $r \ldots 117$

7.24 Subtracted two-pion mass spectra for data and JETSET with and without Bose-Einstein correlations . . . . . . . . . . . . . 119

7.25 Three-particle correlation function . . . . . . . . . . . . 120 


\section{List of Tables}

3.1 LEP statistics used for the Z line shape analysis . . . . . . . . . . . . 19

3.2 Characteristics of the four LEP experiments . . . . . . . . . . . . 22

3.3 Parameters for Jetset . . . . . . . . . . . . . . . . 33

3.4 Parameters for Herwig . . . . . . . . . . . . . . . . 33

5.1 Average hadron multiplicities measured by the four LEP experiments . . . 40

5.2 Average hadron multiplicities measured at LEP in comparison with Monte Carlo models ....................... . . 42

5.3 Average particle multiplicities at lower centre-of-mass energy . . . . . . . 43

6.1 Properties of stable particles and their measured average multiplicities . . . 47

6.2 Properties of $\mathrm{V}^{0}$ particles and their measured average multiplicities . . . . 54

6.3 Properties of particles decaying to photons and their measured average multiplicities. . . . . . . . . . . . . . . 58

6.4 Properties of vector mesons and their measured average multiplicities . . . 62

6.5 Properties of scalar and tensor mesons and their measured average multi-

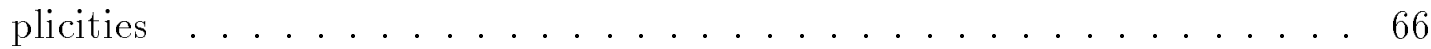

6.6 Properties of non-strange baryons and their measured average multiplicities 69

6.7 Properties of strange baryons and their measured average multiplicities . . 71

6.8 Properties of charmed hadrons and their measured average multiplicities . 75

6.9 Properties of charmonia and bottomonia and their measured average multiplicities......................... 80

6.10 Properties of bottom hadrons and their measured average multiplicities . . 83

7.1 Fitted parameter values for the thermodynamical approach . . . . . . . 90

7.2 Fraction of particles from decay . . . . . . . . . . . . . . 94

7.3 Maximum position $\xi^{*}$ of the $\xi$ distribution . . . . . . . . . . . . . 97

$7.4 \xi^{*}$ for charged particles as function of the centre-of-mass energy . . . . . 100

7.5 Average charged multiplicity in $c \bar{c}$ and $b \bar{b}$ events . . . . . . . . . . . . 105

7.6 Hadron multiplicities measured in $\mathrm{b} \bar{b}$ events and $\mathrm{B}$ hadrons . . . . . . . 106

7.7 Ratio of the charged particle multiplicities in gluon and quark jets . . . . 108

7.8 Average number $\bar{n}_{g \rightarrow c \bar{c}}$ of gluon-splitting to $c \bar{c}$ pairs . . . . . . . . . 110

7.9 Two-particle combinations per hadronic event . . . . . . . . . . . 113

7.10 Bose-Einstein correlations: Chaoticity parameter $\lambda$ and radius $r$ measured at LEP . . . . . . . . . . . . . . . . . 118 


\section{Bibliography}

[1] T. Sjöstrand, Comp. Phys. Comm. 27 (1982) 243;

T. Sjöstrand, Comp. Phys. Comm. 28 (1983) 229;

T. Sjöstrand and M. Bengtson, Comp. Phys. Comm. 43 (1987) 367.

[2] G. Marchesini and B.R. Webber, Nucl. Phys. B310 (1988) 461;

G. Marchesini et al., Comp. Phys. Comm. 67 (1992) 465.

[3] I.G. Knowles, T. Sjöstrand et al., Contribution to the Workshop on Physics at LEP 2: QCD event generators; Eds.: G. Altarelli, T. Sjöstrand and F. Zwirner, CERN yellow report $96-01$ (1996).

[4] D. Buskulic et al. (ALEPH Collab.), Z. Phys. C55 (1992) 209;

G. Rudolph and G. Dissertori, private communication.

[5] (DELPHI Collab.), Contribution to the 28th International Conference on High Energy Physics, Brussels (July 1995), EPS548; K. Hamacher et al., Wuppertal Preprint WU B 95-07 (1995).

[6] B. Adeva et al. (L3 Collab.), Z. Phys. C55 (1992) 39.

[7] M.Z. Akrawy et al. (OPAL Collab.), Z. Phys. C47 (1990) 505.

[8] D. Buskulic et al. (ALEPH Collab.), Phys. Lett. B273 (1991) 181;

D. Buskulic et al. (ALEPH Collab.), Phys. Lett. B357 (1995) 487.

[9] P. Abreu et al. (DELPHI Collab.), Z. Phys. C50 (1991) 185;

P. Abreu et al. (DELPHI Collab.), Phys. Lett. B311 (1993) 408.

[10] B. Adeva et al. (L3 Collab.), Phys. Lett. B259 (1991) 199.

[11] M.Z. Akrawy et al. (OPAL Collab.), Phys. Lett. B247 (1990) 617;

P.D. Acton et al. (OPAL Collab.), Z. Phys. C53 (1992) 539.

[12] D. Buskulic et al. (ALEPH Collab.), Z. Phys. C66 (1995) 355.

[13] R. Akers et al. (OPAL Collab.), Z. Phys. C63 (1994) 181. 
[14] P. van Gemmeren, Diploma thesis, University of Siegen (1995);

A. Böhrer, Presented at the German Physical Society Meeting, Hamburg (March1996);

D. Buskulic et al. (ALEPH Collab.), in preparation (1996).

[15] (DELPHI Collab.), Contribution to the 28th International Conference on High Energy Physics, Brussels (July 1995), EPS542.

[16] M. Acciarri et al. (L3 Collab.), Phys. Lett. B328 (1994) 223.

[17] P. Abreu et al. (DELPHI Collab.), Nucl. Phys. B444 (1995) 3.

[18] D. Buskulic et al. (ALEPH Collab.), Z. Phys. C64 (1994) 361.

[19] P. Abreu et al. (DELPHI Collab.), Z. Phys. C65 (1995) 587.

[20] R. Akers et al. (OPAL Collab.), Z. Phys. C67 (1995) 389.

[21] D. Buskulic et al. (ALEPH Collab.), Phys. Lett. B292 (1992) 210;

(ALEPH Collab.), Contribution to the 27th International Conference on High Energy Physics, Glasgow (July 1994).

[22] (L3 Collab.), Contribution to the 28th International Conference on High Energy Physics, Brussels (July 1995), EPS097.

[23] D. Buskulic et al. (ALEPH Collab.), Z. Phys. C69 (1996) 379.

[24] H. Hepp, Diplomarbeit, Universität Heidelberg (1993);

(ALEPH Collab.), Contribution to the 27th International Conference on High Energy Physics, Glasgow (July 1994).

[25] P.D. Acton et al. (OPAL Collab.), Phys. Lett. B305 (1992) 407.

[26] P. Abreu et al. (DELPHI Collab.), Phys. Lett. B298 (1993) 236.

[27] R. Akers et al. (OPAL Collab.), Z. Phys. C68 (1995) 1.

[28] (DELPHI Collab.), Contribution to the 28th International Conference on High Energy Physics, Brussels (July 1995), EPS556.

[29] P. Abreu et al. (DELPHI Collab.), CERN preprint CERN-PPE-96-026 (1996) submitted to Phys. Lett. B.

[30] P. Abreu et al. (DELPHI Collab.), Phys. Lett. B361 (1995) 207.

[31] G. Alexander et al. (OPAL Collab.), Phys. Lett. B358 (1995) 162.

[32] P. Abreu et al. (DELPHI Collab.), Phys. Lett. B318 (1993) 249. 
[33] G. Alexander et al. (OPAL Collab.), Phys. Lett. B291 (1992) 503;

(OPAL Collab.), Contribution to the 27th International Conference on High Energy Physics, Glasgow (July 1994).

[34] W. Adam et al. (DELPHI Collab.), CERN preprint CERN-PPE-96-012 (1996) submitted to Z. Phys. C.

[35] (OPAL Collab.), Contribution to the 28th International Conference on High Energy Physics, Brussels (July 1995), EPS328; OPAL physics note PN191.

[36] P. Abreu et al. (DELPHI Collab.), Z. Phys. C67 (1995) 543.

[37] (ALEPH Collab.), Contribution to the 28th International Conference on High Energy Physics, Brussels (July 1995), EPS419;

A. Böhrer, Presented at the German Physical Society Meeting, Hamburg (March1996);

S. Black, J. Dann, R. Johnson, ALEPH internal note 96-040 (1996).

[38] D. Buskulic et al. (ALEPH Collab.), Z. Phys. C62 (1994) 1.

[39] P. Abreu et al. (DELPHI Collab.), Z. Phys. C59 (1993) 533.

[40] (OPAL Collab.), Contribution to the 28th International Conference on High Energy Physics, Brussels (July 1995), EPS284.

[41] R. Akers et al. (OPAL Collab.), Z. Phys. C67 (1995) 27.

[42] D. Buskulic et al. (ALEPH Collab.), Phys. Lett. B361 (1995) 221; see also Reference [54].

[43] P. Abreu et al. (DELPHI Collab.), Z. Phys. C61 (1994) 407;

P. Abreu et al. (DELPHI Collab.), CERN preprint CERN-PPE-96-032 (1996) submitted to Z. Phys. C.

[44] P.D. Acton et al. (OPAL Collab.), Phys. Lett. B281 (1992) 394.

[45] D. Buskulic et al. (ALEPH Collab.), Phys. Lett. B295 (1992) 396.

[46] P. Abreu et al. (DELPHI Collab.), Phys. Lett. B341 (1994) 109.

[47] O. Adriani et al. (L3 Collab.), Phys. Lett. B317 (1993) 467.

[48] G. Alexander et al. (OPAL Collab.), CERN preprint CERN-PPE-95-153 (1996) submitted to Z. Phys. C.

[49] (OPAL Collab.), Contribution to the 28th International Conference on High Energy Physics, Brussels (July 1995), EPS286.

[50] D. Buskulic et al. (ALEPH Collab.), Z. Phys. C69 (1996) 393. 
[51] P. Abreu et al. (DELPHI Collab.), CERN preprint CERN-PPE-95-053 (1996) submitted to Z. Phys. C.

[52] M. Acciarri et al. (L3 Collab.), Phys. Lett. B345 (1995) 589.

[53] P. Abreu et al. (DELPHI Collab.), Phys. Lett. B345 (1995) 598;

(DELPHI Collab.), Contribution to the 28th International Conference on High Energy Physics, Brussels (July 1995), EPS563.

[54] D. Buskulic et al. (ALEPH Collab.), CERN preprint CERN-PPE-95-092 (1995) submitted to Z. Phys. C.;

D. Buskulic et al. (ALEPH Collab.), CERN preprint CERN-PPE-96-030 (1996) submitted to Phys. Lett. B.

[55] P. Abreu et al. (DELPHI Collab.), Phys. Lett. B289 (1992) 199.

[56] G. Alexander et al. (OPAL Collab.), CERN preprint CERN-PPE-95-181 (1995) submitted to Phys. Lett. B.

[57] D. Decamp et al. (ALEPH Collab.), Phys. Lett. B278 (1992) 209;

D. Buskulic et al. (ALEPH Collab.), CERN preprint CERN-PPE-96-028 (1996) submitted to Phys. Lett. B.

[58] P. Abreu et al. (DELPHI Collab.), Phys. Lett. B311 (1993) 379.

[59] P.D. Acton et al. (OPAL Collab.), Phys. Lett. B281 (1992) 394.

[60] (DELPHI Collab.), Contribution to the 28th International Conference on High Energy Physics, Brussels (July 1995), EPS565.

[61] P. Abreu et al. (DELPHI Collab.), Z. Phys. C68 (1995) 541.

[62] W. Hofmann, Ann. Rev. Nucl. and Part. Sci. 38 (1988) 279.

[63] G.D. Lafferty, P.I. Reeves and M.R. Whalley, J. Phys. G21 (1995) Supplement 12 A.

[64] M. Schmelling, Phys. Scripta 51 (1995) 683.

[65] Z. Kunszt and P. Nason, in "Z Physics at LEP I", CERN-PPE-89-08, Vol 1 (1989) 373 ;

T. Sjöstrand, in "Z Physics at LEP I", CERN-PPE-89-08, Vol 3 (1989) 143.

[66] D. Schaile, Precision Test of Electro-Weak Interactions, World Scientific Singapore (1995), Ed. P. G. Langacker.

[67] S. Bethke, Contribution to the Workshop on QCD at LEP, Aachen (1994); Eds.: W. Bernreuther and S. Bethke.

[68] LEP Electroweak Working Group and the LEP collaborations, CERN preprint CERN-PPE-95-172 (1995). 
[69] G. Sterman et al., Rev. Mod. Phys. Vol.67, No.1 (1995) 157.

[70] F. Abe et al. (CDF Collab.), Phys. Rev. Lett. 74 (1995) 2626;

S. Abachi et al. (D0 Collab.), Phys. Rev. Lett. 74 (1995) 2632.

[71] S.G. Gorishny, A.L. Kataev and S.A. Larin, Phys. Lett. B212 (1988) 238;

S.G. Gorishny, A.L. Kataev and S.A. Larin, Phys. Lett. B259 (1991) 144;

L.R. Surguladze and M.A. Samuel, Phys. Rev. Lett. 66 (1991) 560, 2416 (E).

[72] S.A. Larin, T. van Ritbergen and J.A.M. Vermaseren, Phys. Lett. B320 (1994) 159.

[73] T. Hebbeker et al., Phys. Lett. B331 (1994) 165.

[74] S. Bethke, Nucl. Phys. B39 (Proc.Suppl.) B,C (1995) 198.

[75] J. Drees, Presented at the 25th International Symposium on Multiparticle Dynamics, Stara Lesna (1995).

[76] T. Sjöstrand, Int. J. Mod. Phys. A3 (1988) 751.

[77] S. Catani, G. Turnock and B.R. Webber Phys. Lett. B272 (1991) 368; and references therein.

[78] R.D. Field and R.P. Feynman, Nucl. Phys. B136 (1978) 1.

[79] L. Montanet et al., Physical Review D50, 1173 (1994) and 1995 off-year partial update for the 1996 edition available on the PDG WWW pages (URL: http://pdg.lbl.gov/).

[80] Y.I. Azimov, Yu.L. Dokshitzer, V.A. Khoze, and S.I. Troyan, Z. Phys. C27 (1985) 65 ;

Y.I. Azimov, Yu.L. Dokshitzer, V.A. Khoze, and S.I. Troyan, Z. Phys. C31 (1986) 213.

[81] C.P. Fong and B.R. Webber, Phys. Lett. B229 (1989) 289;

C.P. Fong and B.R. Webber, Nucl. Phys. B355 (1991) 54;

Yu.L. Dokshitzer, V.A. Khoze, and S.I. Troyan, Z. Phys. C55 (1992) 107.

[82] P.V. Chliapnikov and V.A. Uvarov, Phys Lett. B345 (1995) 313.

[83] F. Becattini, Firenze DFF 224/03/1995 (1995), submitted to Z. Phys. C.

[84] LEP design report, CERN-LEP/84-01 (1984).

[85] SL-Division, CERN, available on the WWW pages (URL: http://www.cern.ch/CERN/Divisions/SL/).

[86] Working Group on LEP Energy, Z. Phys. C66 (1995) 567. 
[87] D. Decamp et al. (ALEPH Collab.), Nucl. Instr. Meth. A294 (1990) 121;

D. Buskulic et al. (ALEPH Collab.), Nucl. Instr. Meth. A360 (1995) 481;

ALEPH Handbook, Ed.: C. Bowdery, ISBN 92-9083-072-7 (1995).

[88] P. Aarnio et al. (DELPHI Collab.), Nucl. Instr. Meth. A303 (1991) 233;

(DELPHI Collab.), Contribution to the 28th International Conference on High Energy Physics, Brussels (July 1995), EPS764;

CERN preprint CERN-PPE-95-194 (1995) submitted to Nucl. Instr Meth.

[89] B. Adeva et al. (L3 Collab.), Nucl. Instr. Meth. A289 (1990) 35;

O. Adriani et al. (L3 Collab.), Phys. Rep. 236 (1993) 1;

F. Beissel et al., Nucl. Instr. Meth. A332 (1993) 33;

B. Acciarri et al. (L3 Collab.), Nucl. Instr. Meth. A351 (1994) 300.

[90] K. Ahmet et al. (OPAL Collab.), Nucl. Instr. Meth. A305 (1991) 275;

P.P. Allport et al. (OPAL Collab.), Nucl. Instr. Meth. A324 (1993) 34;

P.P. Allport et al. (OPAL Collab.), Nucl. Instr. Meth. A346 (1993) 476.

[91] J.-E. Augustin, Contribution to 4th Hellenic School on Elementary Particle Physics, Corfu (Sept. 1992); Eds.: E. N. Gazis et al.

[92] W.J. Stirling, J. Phys. G17 (1991) 1567.

[93] R. Vogl, Dissertation, Universität Innsbruck, Innsbruck (1995).

[94] B. Acciarri et al. (L3 Collab.), CERN preprint CERN-PPE-94-164 (1995) submitted to Phys. Lett. B;

(OPAL Collab.), Contribution to the 28th International Conference on High Energy Physics, Brussels (July 1995), EPS332.

[95] S. Brandt et al.,Phys. Lett. 12 (1964) 57;

E. Fahri, Phys. Rev. Lett. 39 (1977) 1587.

[96] J.D. Bjorken, S. Brodsky, Phys. Rev. D1 (1970) 1416.

[97] G. Rudolph, private communication.

[98] K. Hamacher, private communication.

[99] M. Weierstall, Dissertation, Wuppertal WUB-DIS 95-11.

[100] A.Beddall, Dissertation, University of Sheffield (1995).

[101] J.D. Jackson, Nuovo Cimento 34 (1964) 1644.

[102] H. Albrecht et al., DESY physics report DESY-87-141 (1987).

[103] R. Marshall, Rept. on Prog. in Phys. 52 (1989) 1329;

R. Marshall, Rutherford Appelton Laboratory Preprint RAL-89-021 (1989). 
[104] D. Wegener, Presented at the 21st International Symposium on Multiparticle Dynamics, Wuhan (1991).

[105] S.L. Wu, DESY physics report DESY-84-028 (1984)

[106] A. de Angelis, J. Phys. G19 (1993) 1233.

[107] G.D. Lafferty, Nucl. Instr. Meth. A355 (1995) 541.

[108] T. Sjöstrand, Presented at the 28th International Conference on High Energy Physics, Brussels (July 1995), and private communication.

[109] B. Rensch, Dissertation Universität Heidelberg, Heidelberg (1992).

[110] M. Bowler, Phys. Lett. B180 (1986) 299.

[111] B. Andersson et al., Phys. Rep. 97 (1983) 31.

[112] G. Gustafson, private communication.

[113] H. Albrecht et al. (ARGUS Collab.), Phys. Lett. B230 (1989) 169.

[114] H. Albrecht et al. (ARGUS Collab.), Z. Phys. C52 (1991) 353.

[115] H. Fürstenau, CERN preprint CERN-PPE-94-155, Presented at Tennessee International Symposium on Radiative Corrections: Status and Outlook, Gatlinburg, Tennessee (June 1994); and references therein.

[116] D. Coffman et al. (MARK-III Collab.), Phys. Rev. Lett. 68 (1992) 282.

[117] J.H. Kühn, S. Nussinov and R. Rückl, Z. Phys. C5 (1980) 117;

J.H. Kühn and R. Rückl, Phys. Lett. B135 (1984) 477.

[118] F. Abe et al. (CDF Collab.), Fermilab preprint FERMILAB-PUB-95/271-E (1995) submitted to Phys. Rev. Lett.

[119] M. Cacciari, M. Greco, Phys. Rev. Lett. 73 (1994) 1586;

M. Cacciari et al., Phys. Lett. B356 (1995) 553;

P. Cho, A.K. Leibovich, Caltech preprints CALT-68-1988, CALT-68-2026 (1995);

P. Cho, Caltech preprint CALT-68-2020 (1995).

[120] P. Abreu et al. (DELPHI Collab.), Z. Phys. C69 (1996) 575.

[121] D. Buskulic et al. (ALEPH Collab.), Phys. Lett. B357 (1995) 699.

[122] M. Feindt, CERN preprint CERN-PPE-95-139, Presented at the 6th International Conference on Hadron Spectroscopy, HADRON '95, Manchester, (July 1995).

[123] E.J. Eichten, C.T. Hill and C. Quigg, Phys. Rev. Lett. 71 (1993) 4116. 
[124] S.B. Chun and C.D. Buchanan, Phys. Lett. B308 (1993) 153.

[125] T. Wibig and D. Sobczynska, Phys. Rev. D49 (1994) 2266.

[126] G.D. Lafferty, Manchester preprint, MAN-HEP-95-1.

[127] T. Wibig and D. Sobczynska, Phys. Rev. D50 (1994) 5657.

[128] Y. Pei, CERN preprint CERN-PPE-96-37, submitted to Z. Phys. C.

[129] G. Bocquet et al. (UA1' Collab.), Phys. Lett. B366 (1996) 441;

G. Bocquet et al. (UA1' Collab.), Phys. Lett. B366 (1996) 447.

[130] P. Koch, B. Müller, J. Rafelski, Phys. Rep. 142 (1986) 167.

[131] D. Buskulic et al. (ALEPH Collab.), Phys. Lett. B359 (1995) 236.

[132] B. Adeva et al (L3 Collab.), Phys. Lett. B288 (1992) 395.

[133] Z. Koba, M.B. Nielsen and P. Olesen, Nucl. Phys. B40 (1972) 317;

A.I. Golokhvastov, Sov. J. Nucl. Phys. 27 (1978) 430;

A.I. Golokhvastov, Sov. J. Nucl. Phys. 30 (1979) 128.

[134] DELPHI Collab., Contribution to the 28th International Conference on High Energy Physics, Brussels (July 1995), EPS547.

[135] B.R. Webber, Phys. Lett. B143 (1984) 501.

[136] P.V. Chliapnikov and V.A. Uvarov, Phys Lett. B251 (1990) 192.

[137] P.C. Rowson et al., Phys. Rev. Lett. 54 (1985) 2580;

Y.L. Dokshitzer et al., Basics of Perturbative QCD, (Ed. Frontieres, Paris), ed. J. Tran Than Van (1991);

B.A. Schumm, Y.L. Dokshitzer, V.A. Khoze and D.S. Koetke, Phys. Rev. Lett. 69 (1992) 3025;

V.A. Petrov and A.V. Kisselev, CERN-TH 7318/94;

J.D. de Deus, Phys. Lett. B355 (1995) 539.

[138] J. Chrin, Presented at the 27th International Conference on High Energy Physics, Glasgow, (July 1994) 893;

and references therein.

[139] A. de Angelis, Presented at the 28th International Conference on High Energy Physics, Brussels (July 1995); CERN preprint CERN-PPE-95-135 (1995).

[140] P. Abreu et al. (DELPHI Collab.), Phys. Lett. B347 (1995) 447.

[141] R. Akers et al. (OPAL Collab.), Phys. Lett. B352 (1995) 176.

[142] R. Akers et al. (OPAL Collab.), Z. Phys. C61 (1994) 209. 
[143] K. Abe et al. (SLD Collab.), SLAC preprint SLAC-PUB-95-6924 (1995); (SLD Collab.), Contribution to the 28th International Conference on High Energy Physics, Brussels (July 1995), EPS672.

[144] (DELPHI Collab.), Contribution to the 28th International Conference on High Energy Physics, Brussels (July 1995), EPS542.

[145] (DELPHI Collab.), Contribution to the 28th International Conference on High Energy Physics, Brussels (July 1995), EPS556.

[146] W. Bartel et al. (JADE Collab.), Z. Phys. C33 (1986) 23.

[147] P. Abreu et al. (DELPHI Collab.), Z. Phys. C56 (1992) 63.

[148] J.B. Gaffney and A.H. Mueller, Nucl. Phys. B250 (1985) 109.

[149] J. Fuster and S. Marti, IFIC/95-60 (1995), Presented at the 28th International Conference on High Energy Physics, Brussels (July 1995).

[150] C. Grupen, Siegen preprint SI-95-11, Presented at the 25th International Symposium on Multiparticle Dynamics, Stara Lesna (1995).

[151] D. Buskulic et al. (ALEPH Collab.), Phys. Lett. B346 (1995) 389.

[152] D. Buskulic et al. (ALEPH Collab.), CERN preprint CERN-PPE-95-184 (1994) submitted to Phys. Lett. B.

[153] R. Akers et al. (OPAL Collab.), Z. Phys. C68 (1995) 179.

[154] C. Peterson and T.F. Walsh, Phys. Lett. B91 (1980) 455.

[155] O. Adriani et al. (L3 Collab.), Phys. Lett. B286 (1992) 403;

CERN preprint CERN-PPE-95-182 (1995) submitted to Phys. Lett. B.

[156] R. Akers et al. (OPAL Collab.), Z. Phys. C67 (1995) 27.

[157] M.H. Seymour, Nucl. Phys. B436 (1995) 163.

[158] R. Akers et al. (OPAL Collab.), Phys. Lett. B353 (1995) 595.

[159] M.L. Mangano and P. Nason, Phys. Lett. B285 (1992) 160.

[160] K. Abe et al. (SLD Collab.), SLAC preprint SLAC-PUB-95-6920 (1995); (SLD Collab.), Contribution to the 28th International Conference on High Energy Physics, Brussels (July 1995), EPS205/206.

[161] G. Gustafson and J. Häkkinen, Phys. Lett. B303 (1993) 350.

[162] J.G. Körner, A. Pilaftsis and M.M. Tung, Z. Phys C63 (1994) 575. 
[163] D. Buskulic et al. (ALEPH Collab.), CERN preprint CERN-PPE-96-004 (1996) submitted to Phys. Lett. B.

[164] (DELPHI Collab.), Contribution to the 28th International Conference on High Energy Physics, Brussels (July 1995), EPS707.

[165] D. Buskulic et al. (ALEPH Collab.), Phys. Lett. B365 (1996) 437.

[166] P. Abreu et al. (DELPHI Collab.), Phys. Lett. B318 (1993) 249.

[167] G. Alexander et al. (OPAL Collab.), Phys. Lett. B291 (1992) 503;

P.D. Acton et al. (OPAL Collab.), Phys. Lett. B305 (1993) 415.

[168] D. Decamp et al. (ALEPH Collab.), Z. Phys. C54 (1992) 75.

[169] P. Abreu et al. (DELPHI Collab.), Phys. Lett. B286 (1992) 201.

[170] P. Abreu et al. (DELPHI Collab.), Z. Phys. C63 (1994) 17.

[171] (DELPHI Collab.), Contribution to the 28th International Conference on High Energy Physics, Brussels (July 1995), EPS544.

[172] P.D. Acton et al. (OPAL Collab.), Phys. Lett. B267 (1991) 143.

[173] F. Verbeure, Presented at the 25th International Symposium on Multiparticle Dynamics, Stara Lesna (1995).

[174] S. Haywood, Rutherford Appelton Laboratory Preprint RAL-94-074 (1994).

[175] E.A. de Wolf, Presented at the 24th International Symposium on Multiparticle Dynamics, Vietri sul Mare (1994); Y.F. Wang, L3 internal note 1621 (1994).

[176] P. Abreu et al. (DELPHI Collab.), Presented at the 27th International Conference on High Energy Physics, Glasgow (July 1994).

[177] P.D. Acton et al. (OPAL Collab.), Z. Phys. C56 (1992) 521.

[178] A. Böhrer, ALEPH internal note 94-045 (1994).

[179] P. Abreu et al. (DELPHI Collab.), Phys. Lett. B355 (1995) 415.

[180] B. Andersson, Presented at the 25th International Symposium on Multiparticle Dynamics, Stara Lesna (1995).

[181] A.E. Blinov et al. (MD-1 Collab.), Z. Phys. C69 (1996) 215.

[182] P. Avery et al. (CLEO Collab.), Phys. Rev. D32 (1985) 2294.

[183] I. Juicic et al. (MARK II Collab.), Phys. Rev. D39 (1989) 1.

[184] L. Lönnblad and T. Sjöstrand, Phys. Lett. B351 (1995) 293. 